\title{
Ambiguity Aversion in Algorithmic and High Frequency TRADING
}

by

Ryan Francis Donnelly

A thesis submitted in conformity with the requirements

for the degree of Doctor of Philosophy

Graduate Department of Mathematics

University of Toronto

(C) Copyright 2014 by Ryan Francis Donnelly 


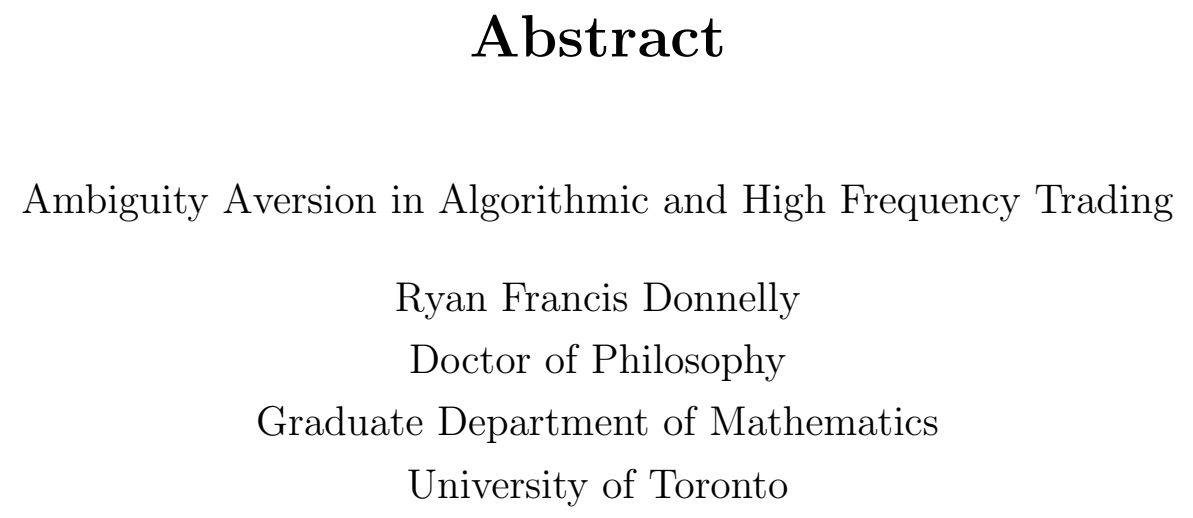

2014

The concept of model uncertainty is one of increasing importance in the field of Mathematical Finance. The main goal of this work is to explore model uncertainty in the specific area of algorithmic and high frequency trading. From a behavioural perspective, model uncertainty naturally leads to the notion of ambiguity aversion - a person's tendency to avoid situations in which randomness plays a role, but the type of randomness itself is uncertain.

Electronic trading algorithms rely heavily on stochastic models of relevant variables, and the act of postulating a specific model creates vulnerabilities and risks due to model misspecification. Within the setting of a commonly used model for limit order and market order dynamics, the effects of protecting oneself against such misspecification in both high frequency market making and liquidation scenarios are investigated. In this case, different types of ambiguity aversion are shown to have different effects on optimal behaviour.

Further in this work, a new reference model is introduced in order to alleviate some practical issues with the original model. This model results in a highly simplified set of allowable trading behaviours, but introduces powerful predictive elements. This work concludes with another investigation of the effects of ambiguity aversion in the context of this new model. 


\section{Acknowledgements}

I must first extend gratitude to my thesis supervisor, Sebastian Jaimungal. I received a tremendous amount of guidance and input from him while producing this work.

I would also like to thank Álvaro Cartea from University College London for intuitive insights and suggestions for improvement.

My thesis committee, consisting of members Luis Seco and Andreas Park, have offered their unique perspectives on some of the interpretations of this work, and it is always encouraging to be challenged to think about the results in different ways.

Two graduate administrators in the Department of Mathematics, Ida Bulat and Jemima Merisca, have been a great assistance since I began my career in this department several years ago. I am thankful for much of the work you put in to making my time here run smoothly.

I am extremely grateful to my friends and family that have supported me through my studies.

Finally, I would like to thank the Bank of Montreal Capital Markets for their financial support and awarding me the Advanced Research Scholarship in 2013. 


\section{Contents}

1 Introduction $\quad 1$

1.1 Background ................................. 1

1.2 Model Uncertainty . . . . . . . . . . . . . . . . . . . . . 2

1.3 Limit Orders and Market Orders . . . . . . . . . . . . . . . 3

1.4 Outline and Contributions ....................... 5

2 Robust Market Making $\quad 8$

2.1 Reference Model . . . . . . . . . . . . . . . . . . . 8

2.1.1 Feedback controls . . . . . . . . . . . . . . . . . . . . 13

2.2 Ambiguity Aversion . . . . . . . . . . . . . . . . . . 15

2.2.1 Ambiguity aversion with respect to market order arrivals . . . . . 17

2.2.2 Ambiguity aversion with respect to fill probability . . . . . . . . . 20

2.2.3 Ambiguity aversion with respect to midprice drift . . . . . . . . . 24

2.3 Mixed Ambiguity . . . . . . . . . . . . . . . . . . . 28

2.4 Closed Form Solutions . . . . . . . . . . . . . . . . . . . . 38 
2.5 Verification Theorem . . . . . . . . . . . . . . . . 40

2.6 Numerical Implementation . . . . . . . . . . . . . . . . . . . . 41

2.7 Simulation results . . . . . . . . . . . . . . . . . . . 42

3 Robust Liquidation $\quad 46$

3.1 Reference Model . . . . . . . . . . . . . . . . . . . 47

3.1.1 Feedback controls ................... 49

3.2 Ambiguity Aversion . . . . . . . . . . . . . . . . . 50

3.2.1 Ambiguity aversion with respect to market order arrivals . . . . . 51

3.2.2 Ambiguity aversion with respect to fill probability . . . . . . . 53

3.2.3 Ambiguity aversion with respect to midprice drift . . . . . . . 55

3.2.4 Mixed Ambiguity . . . . . . . . . . . . . . . 58

3.3 Closed Form Solutions . . . . . . . . . . . . . . . . . . . 59

3.4 Inclusion of Market Orders . . . . . . . . . . . . . . . . . . 62

3.4.1 Feedback controls .................. 63

3.4.2 The effects of ambiguity aversion on market order execution . . . 65

3.4.3 Numerical implementation with market orders . . . . . . . . . 66

4 Volume Order Imbalance $\quad 68$

4.1 Shortcomings of Continuous Model . . . . . . . . . . . . . 68

4.1.1 Tick activity....................... 69

4.2 Imbalance as a Market Predictor . . . . . . . . . . . . . . 71 
4.2.1 Market order activity . . . . . . . . . . . . . 71

4.2 .2 Midprice dynamics . . . . . . . . . . . . . . . . 72

4.3 Modelling Imbalance . . . . . . . . . . . . . . . . . . . 75

4.4 A New Reference Model . . . . . . . . . . . . . . . 76

4.4.1 Feedback controls .................. 79

4.5 Calibration of Imbalance Dependent Factors . . . . . . . . . . . . . . . . 84

5 Imbalance Ambiguity $\quad 90$

5.1 Ambiguity aversion with respect to market order arrivals . . . . . . . . 91

5.2 Ambiguity aversion with respect to jump distribution . . . . . . . . 95

5.3 Ambiguity aversion with respect to imbalance regime changes . . . . . . 100

5.3.1 Transition rates . . . . . . . . . . . . . . 103

5.3.2 Transition probabilities ..................... 105

5.3.3 Penalties due to ambiguity aversion on regime switching . . . . . 108

5.4 Ambiguity aversion with respect to rate of midprice jumps . . . . . . . 111

6 Future Work 116

6.1 Model Uncertainty and Ambiguity Aversion . . . . . . . . . . . . 116

6.2 Limit Order Queues . . . . . . . . . . . . . . . . 117

6.3 Model Improvements . . . . . . . . . . . . . . . . . . . . . . 118

$\begin{array}{ll}\text { A Background Material } & 120\end{array}$ 
A.1 The Hamilton-Jacobi-Bellman PDE . . . . . . . . . . . . . . . . . . . . . 120

A.2 Poisson Random Measures . . . . . . . . . . . . . . . . . . . . 123

A.2.1 Girsanov's Theorem for Poisson random measures . . . . . . . . . 124

A.3 The Transition Groupoid . . . . . . . . . . . . . . . . . . . 127

B Proofs

B.1 Proofs from Chapter $2 \ldots \ldots \ldots \ldots$

B.1.1 Verification Theorem . . . . . . . . . . . . . . . 141

B.2 Proofs from Chapter $3 \ldots \ldots \ldots$. . . . . . . . . . . . 147

B.3 Proofs from Chapter $4 \ldots \ldots \ldots$. . . . . . . . . . . . 151

B.4 Proofs from Chapter $5 \ldots \ldots \ldots \ldots$

Bibliography 


\section{Chapter 1}

\section{Introduction}

\subsection{Background}

Computerized trading in financial markets now accounts for the majority of executed orders in many markets. The absence of human action in these trades requires the development of algorithms which can be automatically executed to satisfy the objectives of financial agents. Possible objectives include, among several others: rebalancing a hedging position in a manner which minimizes transaction costs, optimally liquidating a block of shares while accounting for market impact, or providing liquidity for other market participants while accounting for inventory risk.

A highly quantitative approach is common when attempting to design algorithms with these objectives in mind. The foundations of academic results in such problems is often attributed to Almgren and Chriss (2000). Since then, numerous result have arisen that tackle a wide variety of problems in the field of high-frequency and algorithmic trading. Due to the nature of such problems, a quantitative model of market microstructure is proposed under which the tools of stochastic optimal control can be used to dictate the corresponding optimal trading strategies. Such a microstructure model must take into account the dynamics of the limit order book and market orders, as well as the impact of the agent's trade executions with these dynamics.

The complex nature of microstructure behaviour have given rise to very different modelling choices. Different models are chosen based on the goals of the work in question. 
Some works strive to achieve dynamics for the limit order book that are endogenous based on behaviour of multiple agents. See for example Parlour (1998). Such models often do not lead to realistic dynamics and do not lend themselves to the setting of an optimal stochastic control problem. For a more realistic limit order book model see for example Cont et al. (2010).

This work will focus on models which make simplifying assumptions about the interaction of a single agent's trading strategy with the market. These simplifications allow for more tractable wealth dynamics of the agent, which in turn provide a better setting for the agent to pose and solve a control problem. For examples of such models and associated control problems, see Fodra and Labadie (2012), Guéant et al. (2013), and Guilbaud and Pham (2013a).

\subsection{Model Uncertainty}

In formulating an optimization problem, the optimizing agent must set a goal or objective. In the context of finance, these objectives are influenced by human behaviour, and so the chosen objective should appropriately reflect the typical desires that people express. For example, when it comes to financial investment, most individuals and companies are risk averse; if two opportunities offer the same expected returns, people generally choose the one with less risk (by various different measures of risk). This goes against the paradigm of "fair value" being associated with expected return and has led to the study of utility maximization. A simple example that shows the problem with the old "fair value" paradigm is the St. Petersburg paradox.

If the St. Petersburg paradox is the toy example that exemplifies a general tendency for risk aversion in financial problems, then the Ellsberg paradox illustrates a tendency for ambiguity aversion. A form of the Ellsberg paradox was introduced in Keynes (1921) but popularized in Ellsberg (1961). Similar themes are also covered in Knight (1921). Its conclusion is that humans do not naturally seek to maximize expected wealth, nor do they seek to maximize the expected utility of wealth. Doing either of these requires knowledge of the probability of possible outcomes. If risk aversion is a reaction to the fear of randomness, then ambiguity aversion is a reaction to the fear of unknown probability.

The importance of ambiguity aversion in finance comes from the fact that the theory 
of stochastic processes is so heavily used. As soon as a model for a stochastic process is postulated, all computations will suffer from inaccuracies due to model misspecification. Since an agent's fear of model uncertainty has an effect on their decision process, incorporating ambiguity aversion in a quantitative manner can shed light on its effects on desirable trading strategies or "fair value" computations.

From an abstract standpoint, there are already several results known about the effects of ambiguity aversion in stochastic control problems. See Øksendal and Mataramvura (2008) for a general account of model uncertainty and associated optimal control problems when the underliers are driven by jump diffusions. Also, see Lim and Shanthikumar (2007) for a duality result between ambiguity aversion and risk aversion. When ambiguity aversion is incorporated to a control problem with a relative entropy approach, and risk aversion is dictated by an exponential utility function, the result is that the optimal controls are equivalent in each case.

There is active research on the effects of model uncertainty in financial hedging. For an account of hedging American options in this context, see Bayraktar et al. (2014). And for superhedging of exotic options under model independence, see Fahim and Huang (2014). The natural relationship between hedging and pricing should indicate that effects of model uncertainty on derivatives pricing are also an area of interest. Indeed it is, as seen in an options pricing context in Cont (2006), as well as in Jaimungal and Sigloch (2012) with respect to credit derivatives. Effects of ambiguity aversion on real options have also been investigated, see for example Jaimungal (2011). Each of these works conclude that ambiguity aversion results in similar but distinct effects to risk aversion. This is one of the reasons that motivated the incorporation of ambiguity aversion in high frequency trading contained in this work; to seek the differences between ambiguity aversion and risk aversion in this setting.

\subsection{Limit Orders and Market Orders}

The limit order book (LOB) represents a collection of all interest to buy or sell a specific quantity of an asset for a specific price. The state of the LOB changes throughout the trading day as agents place and cancel limit orders or as incoming market orders are matched with limit orders through a trade execution. Table 1.1 shows a simple example of a LOB at a single point in time. The state of the LOB can also be represented 


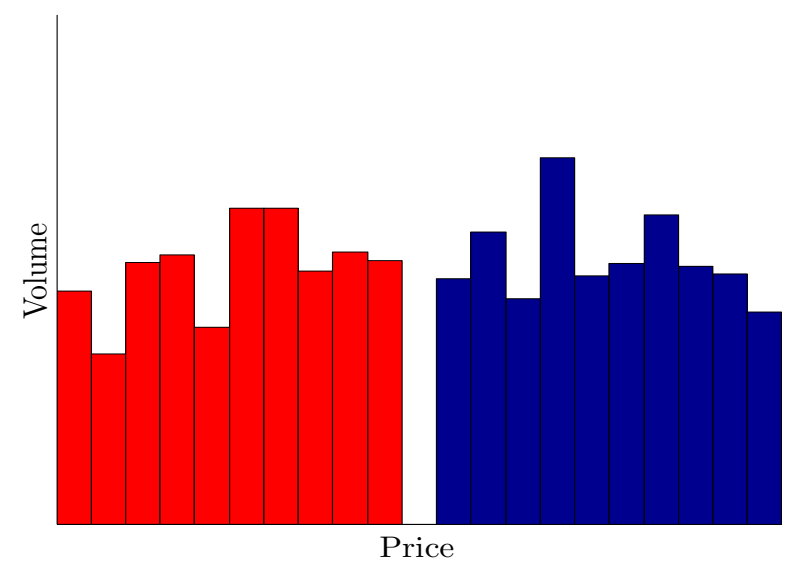

Figure 1.1: Graphical representation of the limit order book

graphically as shown in Figure 1.1.

\begin{tabular}{ccccc}
\hline \hline \multicolumn{2}{c}{ Buy } & Orders & & \multicolumn{2}{c}{ Sell } & Orders \\
\cline { 1 - 2 } \cline { 5 - 6 } Price & Volume & & Price & Volume \\
60 & 80 & & 60.10 & 75 \\
59.90 & 100 & & 60.20 & 75 \\
59.80 & 90 & & 60.30 & 50 \\
59.70 & 50 & & 60.40 & 90 \\
59.60 & 75 & & 60.50 & 80
\end{tabular}

Table 1.1: The limit order book

While limit orders represent standing interest to trade an asset, a market order expresses an agent's desire to execute a trade immediately. Placing a market order of a particular volume $V$ will match the first $V$ shares worth of limit orders in a transaction. With this graphical representation, the interaction between a market order and the limit order book is represented in Figure 1.2.

Many topics of research in high-frequency and algorithmic trading can essentially be framed as how an agent can optimally place limit orders and market orders subject to their objective and microstructure model. 


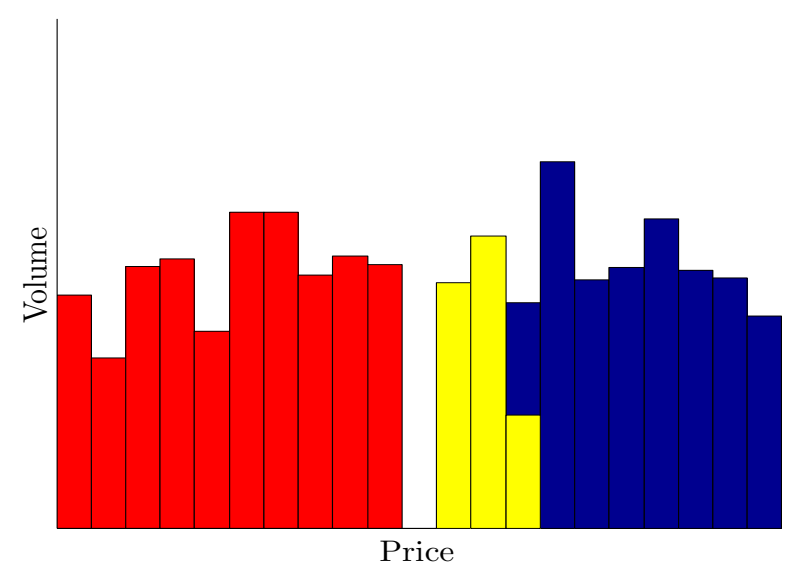

Figure 1.2: A market buy order is matched with the best prices of limit sell orders

\subsection{Outline and Contributions}

In Chapter 2, a model similar to those in Avellaneda and Stoikov (2008), Guilbaud and Pham (2013b), Guéant et al. (2013), Cartea and Jaimungal (2012), and Fodra and Labadie (2012) will be described. Then the agent's ambiguity aversion will be introduced through an embedded control problem. The agent will still attempt to maximize their terminal expected wealth, but several measures that are equivalent to the original measure will be considered as candidates. The class of measures that are considered depends on which factors of the model the agent is ambiguity averse towards. This chapter will consider the drift of the midprice, the rate of market order arrival, and the probability of limit orders being filled as three factors to apply ambiguity. In each case the optimal spreads will be compared to those of an ambiguity neutral agent. Some additional attention will be given to the case of ambiguity on midprice drift due to its equivalence to penalization of inventory holdings.

Although this type of problem has been considered in a very general sense in Øksendal and Mataramvura (2008), this work consists of the first time that this type of analysis is considered in the specific setting of high frequency and algorithmic trading. In particular, the effects of ambiguity aversion on the optimal market making strategies are studied and interpreted from a financial and behavioural perspective.

This chapter will also introduce the notion of mixed ambiguity in which the agent has different levels of ambiguity to different factors of the model. This is the first work in 
which ambiguity aversion is decomposed so that different factors of the model can be weighted with different levels. The three original forms of ambiguity can be considered as specific instances of the mixed case. The proper handling of the measure changes and corresponding entropic penalizations are covered. Under a specific set of parameters, there is a closed form solution to this problem, but it will be shown that classical solutions still exist in all cases, easily leading to a verification theorem. The chapter will conclude with simulation results which illustrate the possible effects of ambiguity aversion on the agent's distribution of terminal wealth for a data generating process that does not come from the reference model.

Chapter 3 will expand on the ideas from Chapter 2 by considering a similar optimal liquidation problem. The reference model in this case is similar with the main difference being that only one side of the limit order book is modelled, and the agent is only able to post one sided limit orders. Although the nature of the model is similar, the behaviour of optimal spreads in the ambiguity neutral case is quite different from that of a market maker. This leads to the question as to whether ambiguity will have similar effects in this problem.

Proceeding as in Chapter 2, ambiguity aversion is introduced with respect to midprice drift, arrival of market orders, and fill probability. After a discussion of how ambiguity aversion on each of these factors affects the agent's trading strategy, the inclusion of market orders is handled. The setting of this chapter is more natural than that of Chapter 2 for the use of market orders. The market order execution strategy is completely determined by a curve through the state space, and so the effects of ambiguity can be investigated through the change in the location of this curve.

The goal of Chapter 4 is to introduce a new reference model for the market making problem. The chapter begins with motivation for introducing this new model based on real data as evidence that the previous model is often unsuitable. An observable stochastic process known as volume order imbalance is introduced and its effects on market behaviour are demonstrated. After introducing this process as another state variable, the ambiguity neutral market making problem is introduced and solved. Although there has been much work done on the study of volume order imbalance and its effects on market behaviour, this is the first work in which a market making problem within the context of an imbalance driven model is studied. The chapter ends with a discussion on how aspects of the model that depend on imbalance are calibrated. 
Chapter 5 performs ambiguity analysis along the lines of Chapters 2 and 3. The factors of the model which are considered in this case are the rate of market order arrivals, the distribution of jumps due to market orders, and the transition rates between imbalance regimes. In a similar way that ambiguity on midprice drift in Chapters 2 and 3 is shown to be equivalent to inventory penalization, ambiguity on the distribution of midprice jumps can be shown to induce minimum and maximum allowable inventory positions by the agent.

Throughout this work, certain types of ambiguity will be shown to give rise to certain risk controls. In a classical context with no consideration of model uncertainty, these risk controls are often incorporated into the optimization problem of the agent, sometimes in a seemingly arbitrary fashion. This work is the first to show that these risk controls can be interpreted as the result of the agent's ambiguity aversion. The specific risk controls that arise are a running inventory penalization and maximum allowable inventory positions. The running inventory penalization comes in two forms: i) a quadratic penalty when the midprice is a diffusion and the agent is averse to ambiguity on the drift (discussed in Sections 2.2.3 and 3.2.3), and ii) a two-sided exponential penalty when the midprice is a pure jump process and the agent is averse to ambiguity on the rate of the jumps (discussed in Section 5.4). The maximum inventory constraint is a result of aversion to ambiguity on the distribution of jump sizes after market orders (discussed in Section 5.2). 


\section{Chapter 2}

\section{Robust Market Making}

Consider an agent which provides liquidity to the market by placing limit orders on both the buy and sell side of the limit order book. Such an agent is referred to as a market maker (MM). In return for placing such orders, the MM expects to make profits each time market orders lift both the MM's buy and sell orders from the book. The magnitude of the profits is equal to the difference in the posted price between the limit buy order and sell order. The MM wishes to post limit orders in such a way that over the course of time, they maximize the profits earned by their trades.

\subsection{Reference Model}

In order to compute the optimal posting strategy of the MM, market dynamics are proposed under which the MM may act. The three ingredients to the model to be considered are: i) the midprice dynamics of the asset, ii) the dynamics of market order (MO) arrivals, and iii) the interaction of MO's and the LOB. This reference model or very similar variations of it are also considered in Avellaneda and Stoikov (2008), Guilbaud and Pham (2013a), Guéant et al. (2013), Cartea and Jaimungal (2012), and Fodra and Labadie (2012). The model in Cartea and Jaimungal (2012) is the one which is most similar to the proposed reference here, so notationally it will follow along the same lines.

Midprice: The midprice $S_{t}$ satisfies: 


$$
d S_{t}=\alpha d t+\sigma d W_{t}
$$

where $\alpha$ and $\sigma>0$ are constants and $\left(W_{t}\right)_{0 \leq t \leq T}$ is a standard Brownian motion.

Market Orders: Let $\mu^{ \pm}$be independent Poisson random measures with compensators $\nu_{\mathbb{P}}^{ \pm}(d y, d t)=\lambda^{ \pm} F^{ \pm}(y) d y d t$, where $F^{ \pm}(y)=\kappa^{ \pm} e^{-\kappa^{ \pm} y}$. Each point in the support of $\mu^{ \pm}$ represents a distinct market order. The $t$ dimension of such a point represents the time at which a market order arrives, and the $y$ dimension represents the maximum distance from the midprice executed by the market order. With this in mind, the number of market buy orders that have arrived is set equal to $M_{t}^{+}=\int_{0}^{t} \int_{0}^{\infty} \mu^{+}(d y, d s)$. Market sell orders are similarly $M_{t}^{-}=\int_{0}^{t} \int_{0}^{\infty} \mu^{-}(d y, d s)$. The time homogeneity of the compensator implies that the number of market orders up to time $t$ are Poisson processes with parameters $\lambda^{ \pm}$. The distribution of the execution price will dictate the probability that limit orders are filled.

Limit Order Placement: The MM elects to place limit orders at a distance $\delta_{t}^{ \pm}$from the midprice. The MM's limit sell (buy) orders are only lifted by a market order if the maximum (minimum) price of the market order is greater (less) than $S_{t}+\delta_{t}^{+}\left(S_{t}-\delta_{t}^{-}\right)$. Thus, the number of sell orders executed by the MM is equal to $N_{t}^{+\delta^{+}}=\int_{0}^{t} \int_{\delta_{s}^{+}}^{\infty} \mu^{+}(d y, d s)$. Similarly, the number of buy orders executed by the MM is $N_{t}^{-\delta^{-}}=\int_{0}^{t} \int_{\delta_{s}^{-}}^{\infty} \mu^{-}(d y, d s)$. The number of filled orders at time $t$ depends on the MM's controls up to time $t$, which is the reason for the superscripts $\delta_{t}^{ \pm}$in the notation. The form of the compensator implies that if the MM has an order placed at a distance $\delta_{t}^{ \pm}$from the midprice, then the probability that a market order at time $t$ will lift the MM's limit order is equal to $e^{-\kappa^{ \pm} \delta_{t}^{ \pm}}$, thus the farther the MM posts from the midprice, the less likely that order is to be filled. The maximum or minimum execution price represents a coupling between both the shape of the limit order book and the volume of market orders. These factors together dictate the maximum or minimum execution price of a market order, and hence, also whether a limit order is filled by a given market order.

Consider the completed filtered probability space $\left(\Omega, \mathbb{F}, \mathcal{F}=\left\{\mathcal{F}_{t}\right\}_{0 \leq t \leq T}, \mathbb{P}\right)$ where $\mathcal{F}$ is generated by the midprice $\left(S_{t}\right)_{0 \leq t \leq T}$, the number of market buy and sell orders that have arrived $\left(M_{t}^{ \pm}\right)_{0 \leq t \leq T}$, and the number of filled limit buy and sell orders $\left(N_{t}^{ \pm \delta^{ \pm}}\right)_{0 \leq t \leq T}$. The MM has control processes $\delta_{t}^{ \pm}$which are non-negative $\mathcal{F}_{t}$-predictable processes. 
With the above stated dynamics, the MM's wealth and inventory processes can also be written. Let $q_{t}^{\delta^{ \pm}}$be the inventory of the MM at time $t$. If a market order lifts the MM's limit sell (buy) order, then the inventory decreases (increases) by one. Thus:

$$
d q_{t}^{\delta^{ \pm}}=d N_{t}^{-\delta^{-}}-d N_{t}^{+\delta^{+}}
$$

If a market buy order lifts the MM's limit sell order, then the MM's wealth increases by a value of $S_{t}+\delta_{t}^{+}$. Similarly, lifting the MM's limit buy order decreases their wealth by $S_{t}-\delta_{t}^{-}$. If $X_{t}^{\delta^{ \pm}}$represents the MM's wealth at time $t$, then the dynamics of $X_{t}^{\delta^{ \pm}}$are:

$$
d X_{t}^{\delta^{ \pm}}=\left(S_{t}+\delta_{t^{-}}^{+}\right) d N_{t}^{+\delta^{+}}-\left(S_{t}-\delta_{t^{-}}^{-}\right) d N_{t}^{-\delta^{-}}
$$

The MM is risk-neutral but capital constrained, so they can not acquire large long or short inventory positions. The MM restricts their inventory such that $-\infty<\underline{q} \leq q_{t}^{\delta^{ \pm}} \leq \bar{q}<\infty$. This is done by setting $\delta_{t}^{+}=\infty$ in the case that $q_{t}^{\delta^{ \pm}}=\underline{q}$, and similarly $\delta_{t}^{-}=\infty$ when $q_{t}^{\delta^{ \pm}}=\bar{q}$. This ensures that the inventory will never deviate beyond the finite bounds. The MM selects the strategy $\left(\delta_{t}^{ \pm}\right)_{0 \leq t \leq T}$ which maximizes the expected terminal wealth:

$$
H(t, x, q, S)=\sup _{\left(\delta_{s}^{ \pm}\right)_{t \leq s \leq T} \in \mathcal{A}} \mathbb{E}_{t, x, q, S}^{\mathbb{P}}\left[X_{T}^{\delta^{ \pm}}+q_{T}^{\delta^{ \pm}}\left(S_{T}-\ell\left(q_{T}^{\delta^{ \pm}}\right)\right)\right]
$$

where $T$ is the terminal time of the strategy, $q_{T}^{\delta^{ \pm}}$final inventory, $\mathbb{E}_{t, x, q, S}^{\mathbb{P}}[\cdot]$ denotes $\mathbb{P}$ expectation conditional on $X_{t^{-}}^{\delta^{ \pm}}=x, q_{t^{-}}^{\delta^{ \pm}}=q$ and $S_{t}=S$, and $\mathcal{A}$ denotes the set of admissible strategies which are non-negative $\mathcal{F}_{t}$-predictable processes such that inventories are bounded above by $\bar{q}$ and below by $\underline{q}$. Moreover, the function $\ell\left(q_{T}^{\delta^{ \pm}}\right)$, with $\ell(0)=0$ and $\ell(q)$ increasing in $q$, is a liquidation penalty which consists of fees and market impact costs when the MM sends an MO to unwind terminal inventory. For example $\ell(\alpha)=\alpha q$ represents a linear impact when liquidating $q$ shares.

Solving the stochastic optimal control problem above can be accomplished by employing a dynamic programming principle and arriving at a Hamiton-Jacobi-Bellman (HJB) equation for $H$ :

$$
\partial_{t} H+\sup _{\delta^{ \pm} \geq 0} \mathcal{L} H=0
$$

where $\mathcal{L}$ is the infinitesimal generator of the underlying state variables given by:

$$
\mathcal{L} H(t, x, q, S)=\alpha \partial_{S} H+\frac{1}{2} \sigma^{2} \partial_{S S} H+\lambda^{+} e^{-\kappa^{+} \delta^{+}} \mathcal{D}^{+} H \mathbb{1}_{q \neq \underline{q}}+\lambda^{-} e^{-\kappa^{-} \delta^{-}} \mathcal{D}^{-} H \mathbb{1}_{q \neq \bar{q}}
$$


where the operators $\mathcal{D}^{ \pm}$act as follows:

$$
\mathcal{D}^{ \pm} H=H\left(t, x \pm\left(S \pm \delta^{ \pm}\right), q \mp 1, S\right)-H(t, x, q, S)
$$

and subject to the terminal condition

$$
H(T, x, q, S)=x+q(S-\ell(q))
$$

where $x, S$, and $q$ are the quantities at $t^{-}$(and not $t$ ). Intuitively, the terms containing the operators $D^{ \pm}$represent the expected rate of change of the value function when the MM posts orders at $\delta^{ \pm}$. By placing a limit sell order at $\delta^{+}$, the change in the value function should this order be filled is equal to $D^{+} H$, and this happens at an expected rate of $\lambda^{+} e^{-\kappa^{+} \delta^{+}}$.

To verify that equations (2.1) and (2.3) hold, proceed as follows: for some choice of the control processes $\delta_{t}^{ \pm}$, define performance criteria:

$$
V^{\delta^{ \pm}}(t, x, q, S)=\mathbb{E}_{t, x, q, S}^{\mathbb{P}}\left[X_{T}^{\delta^{ \pm}}+q_{T}^{\delta^{ \pm}}\left(S_{T}-\ell\left(q_{T}^{\delta^{ \pm}}\right)\right)\right]
$$

This means $H(t, x, q, S)=\sup _{\delta^{ \pm} \in \mathcal{A}} V^{\delta^{ \pm}}(t, x, q, S)$. Clearly $V^{\delta^{ \pm}}(T, x, q, S)=x+q(S-\ell(q))$ for any choice of $\delta^{ \pm}$, and so the boundary conditions (2.3) are satisfied. Now suppose $\delta^{* \pm}$ is an optimal control so that $H(t, x, q, S)=V^{\delta^{* \pm}}(t, x, q, S)$. Fix $t$ and $h$ and define $\tilde{\delta}_{u}^{ \pm}$as:

$$
\tilde{\delta}_{u}^{ \pm}= \begin{cases}\bar{\delta}^{ \pm} & t \leq u \leq t+h \\ \delta_{u}^{* \pm} & u>t+h\end{cases}
$$

In other words, the control $\tilde{\delta}_{t}^{ \pm}$is optimal after time $t+h$, but between $t$ and $t+h$ it is an arbitrary constant. This means:

$$
\begin{aligned}
H(t, x, q, S) & \geq V^{\tilde{\delta}^{ \pm}}(t, x, q, S) \\
& \geq \mathbb{E}_{t, x, q, S}^{\mathbb{P}}\left[X_{T}^{\tilde{\delta}^{ \pm}}+q_{T}^{\tilde{\delta}^{ \pm}}\left(S_{T}-\ell\left(q_{T}^{\tilde{\delta}^{ \pm}}\right)\right)\right] \\
& =\mathbb{E}_{t, x, q, S}^{\mathbb{P}}\left[\mathbb{E}_{t+h, X_{t+h}^{\tilde{\delta}^{ \pm}}, q_{t+h}^{\tilde{\delta}^{ \pm}}, S_{t+h}}^{\mathbb{P}}\left[X_{T}^{\tilde{\delta}^{ \pm}}+q_{T}^{\tilde{\delta}^{ \pm}}\left(S_{T}-\ell\left(q_{T}^{\tilde{\delta}^{ \pm}}\right)\right)\right]\right] \\
& =\mathbb{E}_{t, x, q, S}^{\mathbb{P}}\left[H\left(t+h, X_{t+h}^{\tilde{\delta}^{ \pm}}, q_{t+h}^{\tilde{\delta}^{ \pm}}, S_{t+h}\right)\right]
\end{aligned}
$$


where the last equality follows from the fact that the control is optimal from time $t+h$ onward. By rearranging this inequality and applying Ito' Lemma:

$$
\begin{aligned}
& 0 \geq \mathbb{E}_{t, x, q, S}^{\mathbb{P}}[\left.H\left(t+h, X_{t+h}^{\tilde{\delta}^{ \pm}}, q_{t+h}^{\tilde{\delta}^{ \pm}}, S_{t+h}\right)-H(t, x, q, S)\right] \\
&=\mathbb{E}_{t, x, q, S}^{\mathbb{P}}\left[\int_{t}^{t+h}\left(\partial_{t}+\alpha \partial_{S}+\frac{1}{2} \sigma^{2} \partial_{S S}\right) H\left(u, X_{u}^{\tilde{\delta}^{ \pm}}, q_{u}^{\tilde{\delta}^{ \pm}}, S_{u}\right) d u\right. \\
&+\int_{t}^{t+h} \sigma \partial_{S} H\left(u, X_{u}^{\tilde{\delta}^{ \pm}}, q_{u}^{\tilde{\delta}^{ \pm}}, S_{u}\right) d W_{u} \\
&+\int_{t}^{t+h} \int_{\bar{\delta}^{+}}^{\infty} \mathcal{D}^{+} H\left(u, X_{u}^{\tilde{\delta}^{ \pm}}, q_{u}^{\tilde{\delta}^{ \pm}}, S_{u}\right) \mathbb{1}_{q_{u} \neq^{\tilde{\delta}^{ \pm}} \underline{q}} \mu^{+}(d y, d u) \\
&\left.+\int_{t}^{t+h} \int_{\bar{\delta}^{-}}^{\infty} \mathcal{D}^{-} H\left(u, X_{u}^{\tilde{\delta}^{ \pm}}, q_{u}^{\tilde{\delta}^{ \pm}}, S_{u}\right) \mathbb{1}_{q_{u}^{\tilde{\delta}} \neq \bar{q}^{ \pm}} \mu^{-}(d y, d u)\right] \\
&=\mathbb{E}_{t, x, q, S}^{\mathbb{P}}\left[\int_{t}^{t+h}\left(\partial_{t}+\alpha \partial_{S}+\frac{1}{2} \sigma^{2} \partial_{S S}\right) H\left(u, X_{u}^{\tilde{\delta}^{ \pm}}, q_{u}^{\tilde{\delta}^{ \pm}}, S_{u}\right) d u\right. \\
&+\int_{t}^{t+h} \lambda^{+} e^{-\kappa^{+} \bar{\delta}^{+}} \mathcal{D}^{+} H\left(u, X_{u}^{\tilde{\delta}^{ \pm}}, q_{u}^{\tilde{\delta}^{ \pm}}, S_{u}\right) \mathbb{1}_{q_{u}^{\tilde{\delta}^{ \pm}} \neq \underline{q}} d u \\
&\left.+\int_{t}^{t+h} \lambda^{-} e^{-\kappa^{-} \bar{\delta}^{-}} \mathcal{D}^{-} H\left(u, X_{u}^{\tilde{\delta}^{ \pm}}, q_{u}^{\tilde{\delta}^{ \pm}}, S_{u}\right) \mathbb{1}_{q_{u}^{\tilde{\delta}^{ \pm}} \neq \bar{q}} d u\right]
\end{aligned}
$$

Later in this chapter (specifically Section 2.5), regularity of the function $H$ with respect to $S$ and $t$ will be shown to justify the existence of the above derivatives. Further, the form of $H$ discussed later and boundedness of the process $q_{t}^{\tilde{\delta}^{ \pm}}$allows for the expectation of the Brownian term to vanish. Lastly, Proposition 10 along with boundedness of $q_{t}^{\tilde{\delta}^{ \pm}}$ and $t \in[0, T]$ allow for $\mu^{ \pm}$to be replaced with $\nu^{ \pm}$within the expectation. Dividing both sides of this inequality by $h$ and taking $h \rightarrow 0$ gives:

$$
0 \geq\left(\partial_{t}+\alpha \partial_{S}+\frac{1}{2} \sigma^{2} \partial_{S S}\right) H+\lambda^{+} e^{-\kappa^{+} \bar{\delta}^{+}} \mathcal{D}^{+} H \mathbb{1}_{q \neq \underline{q}}+\lambda^{-} e^{-\kappa^{-} \bar{\delta}^{-}} \mathcal{D}^{-} H \mathbb{1}_{q \neq \underline{q}}
$$

Since this holds for all $\bar{\delta}^{ \pm} \geq 0$, a supremum over these values preserves the inequality:

$$
0 \geq\left(\partial_{t}+\alpha \partial_{S}+\frac{1}{2} \sigma^{2} \partial_{S S}\right) H+\sup _{\delta^{ \pm} \geq 0}\left\{\lambda^{+} e^{-\kappa^{+} \bar{\delta}^{+}} \mathcal{D}^{+} H \mathbb{1}_{q \neq \underline{q}}+\lambda^{-} e^{-\kappa^{-} \bar{\delta}^{-}} \mathcal{D}^{-} H \mathbb{1}_{q \neq \underline{q}}\right\}
$$

If $\tilde{\delta}^{ \pm}$is chosen to be optimal, that is $\bar{\delta}^{ \pm}=\delta^{* \pm}$, then all of the inequalities in the preceding 
argument are actually equalities, and therefore:

$$
0=\left(\partial_{t}+\alpha \partial_{S}+\frac{1}{2} \sigma^{2} \partial_{S S}\right) H+\sup _{\delta \pm \geq 0}\left\{\lambda^{+} e^{-\kappa^{+} \bar{\delta}^{+}} \mathcal{D}^{+} H \mathbb{1}_{q \neq \underline{q}}+\lambda^{-} e^{-\kappa^{-} \bar{\delta}^{-}} \mathcal{D}^{-} H \mathbb{1}_{q \neq \underline{q}}\right\}
$$

Thus equation (2.1) is satisfied. For a more detailed argument, see Section A.1. For the remainder of this work, the explicit dependence of variables on the control processes $\delta^{ \pm}$ will not be indicated with superscripts to improve readability. It is to be understood that many processes involved may depend on the controls.

\subsubsection{Feedback controls}

The form of the HJB equation and terminal conditions allow for a convenient ansatz: $H(t, x, q, S)=x+q S+h_{q}(t)$. Substituting this into equation 2.1 gives a system of equations for $h_{q}(t)$ :

$$
\begin{aligned}
\partial_{t} h_{q}+\alpha q & +\sup _{\delta^{+} \geq 0}\left\{\lambda^{+} e^{-\kappa^{+} \delta^{+}}\left(\delta^{+}+h_{q-1}-h_{q}\right)\right\} \mathbb{1}_{q \neq \underline{q}} \\
& +\sup _{\delta^{-} \geq 0}\left\{\lambda^{-} e^{-\kappa^{-} \delta^{-}}\left(\delta^{-}+h_{q+1}-h_{q}\right)\right\} \mathbb{1}_{q \neq \bar{q}}=0,
\end{aligned}
$$

subject to terminal conditions $h_{q}(T)=-q \ell(q)$. The function $h_{q}(t)$ may be interpreted in the following way: since $H(t, x, q, S)=x+q S+h_{q}(t)$ is the total value had by the market maker, and $x+q S$ is the value of the market maker's cash and inventory holdings, $h_{q}(t)$ is interpreted as the value that the market maker draws from the ability to post limit orders. It is in a sense the value of the pure optionality component of the market maker's situation.

Proposition 1 (Optimal Feedback Controls). The optimal feedback controls of the HJB equation (2.4) are given by

$$
\begin{aligned}
& \delta_{q}^{+*}(t)=\left(\frac{1}{\kappa^{+}}-h_{q-1}(t)+h_{q}(t)\right)_{+}, \quad q \neq \underline{q}, \\
& \delta_{q}^{-*}(t)=\left(\frac{1}{\kappa^{-}}-h_{q+1}(t)+h_{q}(t)\right)_{+}, \quad q \neq \bar{q} .
\end{aligned}
$$

where $(x)_{+}=\max (x, 0)$. 
Proof. Apply first order conditions to each supremum term in equation (2.4). For the first term, this gives:

$$
\begin{aligned}
-\lambda^{+} \kappa^{+} e^{-\kappa^{+} \delta^{+}}\left(\delta^{+}+h_{q-1}-h_{q}\right)+\lambda^{+} e^{-\kappa^{+} \delta^{+}} & =0 \\
-\kappa^{+}\left(\delta^{+}+h_{q-1}-h_{q}\right)+1 & =0 \\
\delta^{+} & =\frac{1}{\kappa^{+}}-h_{q-1}+h_{q}
\end{aligned}
$$

Suppose this quantity is positive. Then the second derivative of this term is equal to:

$$
\begin{aligned}
& -\lambda^{+} \kappa^{+2} e^{-\kappa^{+} \delta^{+}}\left(\delta^{+}+h_{q-1}-h_{q}\right)-2 \lambda^{+} \kappa^{+} e^{-\kappa^{+} \delta^{+}} \\
= & -e^{-\kappa^{+}\left(\frac{1}{\kappa^{+}}-h_{q-1}+h_{q}\right)} \lambda^{+} \kappa^{+} \\
< & 0
\end{aligned}
$$

and so the value of $\delta^{+}$does indeed yield a maximum. Now suppose the feedback expression is negative. Then, since $\delta^{+}$is constrained to being non-negative, the feedback expression does not yield an admissible strategy. Rather, computing the first derivative of the term for $\delta^{+} \geq 0$ gives:

$$
\begin{aligned}
& -\lambda^{+} \kappa^{+} e^{-\kappa^{+} \delta^{+}}\left(\delta^{+}+h_{q-1}-h_{q}\right)+\lambda^{+} e^{-\kappa^{+} \delta^{+}} \\
= & \left(-\lambda^{+} \kappa^{+}\left(\delta+h_{q-1}-h_{q}\right)+\lambda\right) e^{-\kappa^{+} \delta^{+}} \\
< & 0
\end{aligned}
$$

where the last inequality comes from $\frac{1}{\kappa^{+}}-h_{q-1}+h_{q}<0$. Since the derivative is negative for all $\delta^{+}>0$, there must be a maximum at $\delta^{+}=0$, and so $\delta^{+*}(t, q)=\left(\frac{1}{\kappa^{+}}-h_{q-1}(t)+h_{q}(t)\right)_{+}$. The proof is identical for the second supremum term.

As long as the stated feedback controls remain positive ${ }^{1}$, they can be substituted into equation (2.4) to obtain a non-linear system of equations for $h_{q}(t)$ :

$$
\left.\partial_{t} h_{q}+\alpha q+\frac{\lambda^{+}}{\kappa^{+}} e^{-\kappa^{+}\left(\frac{1}{\kappa^{+}}-h_{q-1}+h_{q}\right)} \mathbb{1}_{q \neq \underline{q}}+\frac{\lambda^{-}}{\kappa^{-}} e^{-\kappa^{-}\left(\frac{1}{\kappa^{-}}-h_{q+1}+h_{q}\right.}\right)_{\mathbb{1}_{q \neq \bar{q}}}=0,
$$

along with terminal conditions $h_{q}(T)=-q \ell(q)$. Under certain circumstances, there

\footnotetext{
${ }^{1}$ Unfortunately, there is no way to determine conclusively whether the spreads remain positive for all $t \in[0, T]$. It is easy to see that for large values of $\kappa^{ \pm}$and $\theta$ the feedback expression may become negative for some values of $q$ at $t=T$. Asymptotic expressions for the spreads at $t$ exist for large $T$ see Cartea and Jaimungal (2012) for this analysis - but again this assumes that they remain positive for all $t \in[0, T]$.
} 
is a closed form analytical solution for $h_{q}(t)$ (discussed in Section 2.4). Note that in Proposition 1 and equation (2.4), $\sigma$ does not affect the solution to the value function or the optimal strategy. When ambiguity on the drift of the midprice is considered, dependence on $\sigma$ will arise in the solution.

In Figure 2.1, the optimal sell spread is shown in the ten seconds approaching maturity. The optimal buy spread is not shown due to the symmetry present between the optimal buy and sell spreads. This will be discussed further in Section 2.4. Note that the time

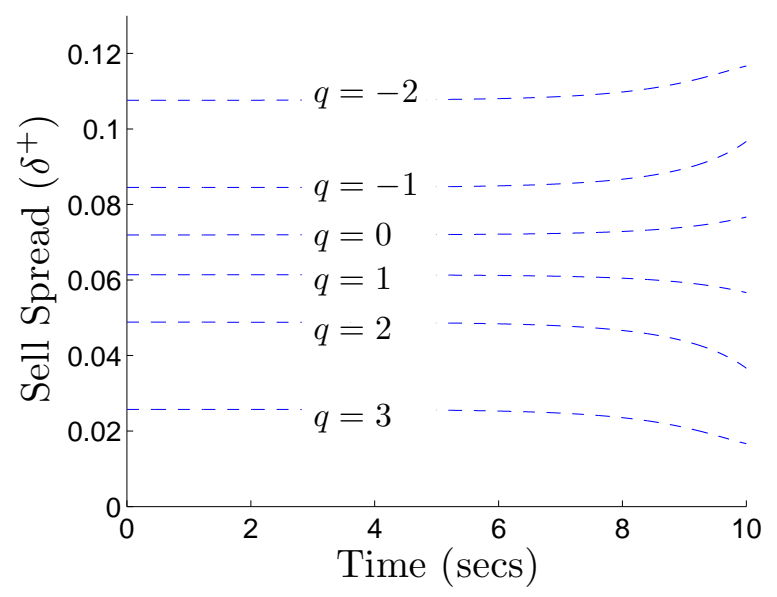

Figure 2.1: Optimal sell spread for an ambiguity neutral agent. Parameter values are $\kappa^{ \pm}=15, \lambda^{ \pm}=2, \sigma=0.01, \alpha=0, \ell(q)=\theta q, \theta=0.01, \bar{q}=-\underline{q}=3$ and $T=10$ seconds.

dependence of $\delta_{q}^{ \pm *}$ is most significant as time approaches maturity. For a discussion of the asymptotic behaviour of $\delta_{q}^{ \pm *}$ when time to maturity is large, see Cartea and Jaimungal (2012).

\subsection{Ambiguity Aversion}

The method in which the MM accounts for model misspecification is as follows: they consider a class of probability measures $\mathcal{Q}$ that are equivalent to the reference measure $\mathbb{P}$. The MM then introduces a penalty function in the optimization problem which represents the cost of rejecting the reference measure $\mathbb{P}$ in favour of the candidate measure $\mathbb{Q}$. The class of measures to be considered will depend on which aspects of the model the MM is 
ambiguity averse towards. A popular choice for the penalty function is entropic penalty, which will be weighted with respect to the MM's level of ambiguity aversion $\varphi$.

The MM will choose a strategy which is robust to model misspecification by maximizing expected penalized terminal wealth with respect to all admissible market making strategies, while simultaneously minimizing expected penalized terminal wealth with respect to all candidate measures. The optimization problem then becomes:

$$
H(t, x, q, S ; \varphi)=\sup _{\left(\delta_{u}^{ \pm}\right)_{t \leq u \leq T} \in \mathcal{A}} \inf _{\mathbb{Q} \in \mathcal{Q}} \mathbb{E}_{t, x, q, S}^{\mathbb{Q}}\left[X_{T}+q_{T}\left(S_{T}-\ell\left(q_{T}\right)\right)+\mathcal{H}_{t, T}[\mathbb{Q} \mid \mathbb{P}]\right]
$$

where

$$
\mathcal{H}_{t, T}[\mathbb{Q} \mid \mathbb{P}]=\frac{1}{\varphi} \log \left\{\left(\frac{d \mathbb{Q}}{d \mathbb{P}}\right)_{T} /\left(\frac{d \mathbb{Q}}{d \mathbb{P}}\right)_{t}\right\}
$$

The constant $\varphi>0$ represents the MM's level of ambiguity aversion in the following sense: note that if $\varphi$ is large, then deviations of $\mathbb{Q}$ from $\mathbb{P}$ have little effect on the optimization problem, and so the MM essentially considers all candidate measures with equal weight and behaves as if the market is operating under a worst case scenario; the MM has little confidence in their reference model. On the other hand, if $\varphi$ is small, then even a small deviation of $\mathbb{Q}$ from $\mathbb{P}$ induces a large penalty, and so the MM behaves according to a measure which is very close to $\mathbb{P}$; the $\mathrm{MM}$ is very confident in their reference measure.

The addition of this penalty function modifies the associated HJB equation to:

$$
\partial_{t} H+\sup _{\delta^{ \pm} \geq 0} \inf _{\mathbb{Q} \in \mathcal{Q}}\left\{\mathcal{L}^{\mathbb{Q}} H-\partial_{t} \mathbb{E}_{t, x, q, S}^{\mathbb{Q}}\left[\mathcal{H}_{t, T}[\mathbb{Q} \mid \mathbb{P}]\right]\right\}=0
$$

with terminal conditions $H(T, x, q, S ; \varphi)=x+q(S-\ell(q))$ and where $\mathcal{L}^{\mathbb{Q}}$ is the infinitesimal generator of the underlying state variables in the measure $\mathbb{Q}$. The agent's aversion to model uncertainty is considered with respect to three aspects of the reference model: i) the rate of arrival of market orders, ii) the probability of limit order fill, and iii) the drift of the midprice. This is done by appropriately selecting the class $\mathcal{Q}$ to contain only measures in which the corresponding factor of the model changes. Each of these choices will give rise to a different expressions for $\mathcal{L}^{\mathbb{Q}}$ and $\mathbb{E}_{t, x, q, S}^{\mathbb{Q}}\left[\mathcal{H}_{t, T}[\mathbb{Q} \mid \mathbb{P}]\right]$. 


\subsubsection{Ambiguity aversion with respect to market order arrivals}

In order to consider the MM's aversion to the rate of market order arrival, introduce a class of equivalent measures through the Radon-Nikodym derivative

$$
\left(\frac{d \mathbb{Q}(g)}{d \mathbb{P}}\right)=\prod_{i=+,-} \exp \left\{-\int_{0}^{T} \int_{0}^{\infty}\left(e^{g_{t}^{i}}-1\right) \nu_{\mathbb{P}}^{i}(d y, d t)+\int_{0}^{T} \int_{0}^{\infty} g_{t}^{i} \mu^{i}(d y, d t)\right\}
$$

In the new measure $\mathbb{Q}(g)$, the compensator of $\mu^{ \pm}(d y, d t)$ is $\nu_{\mathbb{Q}}^{ \pm}(d y, d t)=e^{g_{t}^{ \pm}} \nu_{\mathbb{P}}^{ \pm}(d y, d t)$ (see Section A.2 or Jacod and Shiryaev (1987) for more details). The processes $g_{t}^{ \pm}$are not allowed to depend on $y$ which means the distribution of the maximum execution price of market orders is unchanged, only their instantaneous rate of arrival has changed and it is now $e^{g_{t}^{ \pm}} \lambda^{ \pm}$. The full class of candidate measures that the MM considers in this setting is:

$$
\mathcal{Q}^{\lambda}=\left\{\mathbb{Q}(g): g^{ \pm} \text {are } \mathcal{F}_{t} \text {-predictable, Markov, and bounded }\right\}
$$

The entropic penalty for this class of measures satisfies (see again Section A.2):

$$
\begin{aligned}
\mathbb{E}_{t, x, q, S}^{\mathbb{Q}(g)}\left[\mathcal{H}_{t, T}^{\varphi_{\lambda}}[\mathbb{Q}(g) \mid \mathbb{P}]\right] & =\frac{1}{\varphi_{\lambda}} \mathbb{E}_{t, x, q, S}^{\mathbb{Q}(g)}\left[\sum_{i=+,-} \int_{t}^{T} \int_{0}^{\infty}\left(e^{g_{u}^{i}}\left(g_{u}^{i}-1\right)+1\right) \nu_{\mathbb{P}}^{i}(d y, d u)\right] \\
& =\frac{1}{\varphi_{\lambda}} \mathbb{E}_{t, x, q, S}^{\mathbb{Q}(g)}\left[\sum_{i=+,-} \lambda^{i} \int_{t}^{T}\left(e^{g_{u}^{i}}\left(g_{u}^{i}-1\right)+1\right) d u\right],
\end{aligned}
$$

and the infinitesimal generator $\mathcal{L}^{\mathbb{Q}(g)}$ is given by:

$$
\begin{aligned}
\mathcal{L}^{\mathbb{Q}(g)} H^{\lambda}\left(t, x, q, S ; \varphi_{\lambda}\right)= & \alpha \partial_{S} H^{\lambda}+\frac{1}{2} \sigma^{2} \partial_{S S} H^{\lambda} \\
& +\lambda^{+} e^{g_{t}^{+}} e^{-\kappa^{+} \delta^{+}} \mathcal{D}^{+} H^{\lambda} \mathbb{1}_{q \neq \underline{q}}+\lambda^{-} e^{g_{t}^{-}} e^{-\kappa^{-} \delta^{-}} \mathcal{D}^{-} H^{\lambda} \mathbb{1}_{q \neq \bar{q}}
\end{aligned}
$$

Making the same ansatz as in the ambiguity neutral case, $H^{\lambda}\left(t, x, q, s ; \varphi_{\lambda}\right)=x+q S+$ $h_{q}^{\varphi_{\lambda}}(t)$, and substituting the infinitesimal generator and penalty function into equation 
(2.6) gives the following:

$$
\begin{aligned}
\partial_{t} h_{q}^{\varphi_{\lambda}}+\alpha q+ & \sup _{\delta^{+} \geq 0} \inf _{g^{+}}\left\{\lambda^{+} e^{g^{+}} e^{-\kappa^{+} \delta^{+}}\left(\delta^{+}+h_{q-1}^{\varphi_{\lambda}}-h_{q}^{\varphi_{\lambda}}\right) \mathbb{1}_{q \neq \underline{q}}\right. \\
& \left.+\frac{1}{\varphi_{\lambda}} \lambda^{+}\left(e^{g^{+}}\left(g^{+}-1\right)+1\right)\right\} \\
+\sup _{\delta^{-} \geq 0} \inf _{g^{-}}\left\{\lambda^{-} e^{g^{-}} e^{-\kappa^{-} \delta^{-}}\left(\delta^{-}+h_{q+1}^{\varphi_{\lambda}}-h_{q}^{\varphi_{\lambda}}\right) \mathbb{1}_{q \neq \bar{q}}\right. & \\
& \left.+\frac{1}{\varphi_{\lambda}} \lambda^{-}\left(e^{g^{-}}\left(g^{-}-1\right)+1\right)\right\}=0, \\
h^{\varphi_{\lambda}}(T, q) & =-q \ell(q) .
\end{aligned}
$$

Proposition 2 (Optimal Feedback Controls). The optimal feedback controls of equation (2.8) are given by

$$
\begin{aligned}
& \delta^{+*}\left(t, q ; \varphi_{\lambda}\right)=\left(\frac{1}{\kappa^{+}}-h_{q-1}^{\varphi_{\lambda}}(t)+h_{q}^{\varphi_{\lambda}}(t)\right)_{+}, q \neq \underline{q}, \\
& \delta^{-*}\left(t, q ; \varphi_{\lambda}\right)=\left(\frac{1}{\kappa^{-}}-h_{q+1}^{\varphi_{\lambda}}(t)+h_{q}^{\varphi_{\lambda}}(t)\right)_{+}, q \neq \bar{q}, \\
& g^{ \pm *}\left(t, q ; \varphi_{\lambda}\right)=-\varphi_{\lambda} e^{-\kappa^{ \pm} \delta^{ \pm *}\left(t, q ; \varphi_{\lambda}\right)}\left(\delta^{ \pm *}\left(t, q ; \varphi_{\lambda}\right)+h_{q \mp 1}^{\varphi_{\lambda}}(t)-h_{q}^{\varphi_{\lambda}}(t)\right) .
\end{aligned}
$$

Proof. See Appendix B.1.

\section{Discussion and Financial Interpretation}

Note that as $\varphi_{\lambda} \rightarrow 0$, the optimal control $g^{ \pm *} \rightarrow 0$ which corresponds to forcing $\mathbb{Q}=\mathbb{P}$. This is consistent with the heavy penalization representing a MM that is very confident in the reference model. Also note that $g^{ \pm *}$ is always negative. This indicates that the MM's fear towards model misspecification is that market orders arrive at a slower rate $\lambda^{ \pm} e^{g_{t}^{ \pm}}<\lambda^{ \pm}$. Finally, note that as $\delta^{ \pm} \rightarrow \infty, g^{ \pm *} \rightarrow 0$. This is due to the fact that if the MM posts very far from the midprice, changing the rate of market order arrivals will have no effect on the performance. 
Substituting the optimal $g^{ \pm *}\left(t, q, ; \varphi_{\lambda}\right)$ into equation (2.8) results in the following:

$$
\begin{aligned}
\partial_{t} h_{q}^{\varphi_{\lambda}}+\alpha q & +\sup _{\delta^{+} \geq 0}\left\{\frac{\lambda^{+}}{\varphi_{\lambda}}\left(1-e^{-\varphi_{\lambda} e^{-\kappa^{+} \delta^{+}}\left(\delta^{+}+h_{q-1}^{\varphi_{\lambda}}-h_{q}^{\varphi_{\lambda}}\right)}\right)\right\} \mathbb{1}_{q \neq \underline{q}} \\
& +\sup _{\delta^{-} \geq 0}\left\{\frac{\lambda^{-}}{\varphi_{\lambda}}\left(1-e^{-\varphi_{\lambda} e^{-\kappa^{-} \delta^{-}}\left(\delta^{-}+h_{q+1}^{\varphi_{\lambda}}-h_{q}^{\varphi_{\lambda}}\right)}\right)\right\} \mathbb{1}_{q \neq \bar{q}}=0
\end{aligned}
$$

There is no known analytical representation for a solution to this equation, but a classical solution does exist (see Proposition 10). Solving the equation numerically allows for a comparison of the optimal market making strategy to that of the reference model in Figure 2.2. The effective market order arrival rates $\left(\lambda^{ \pm} e^{g_{t}^{ \pm}}\right)$in the measure corresponding to
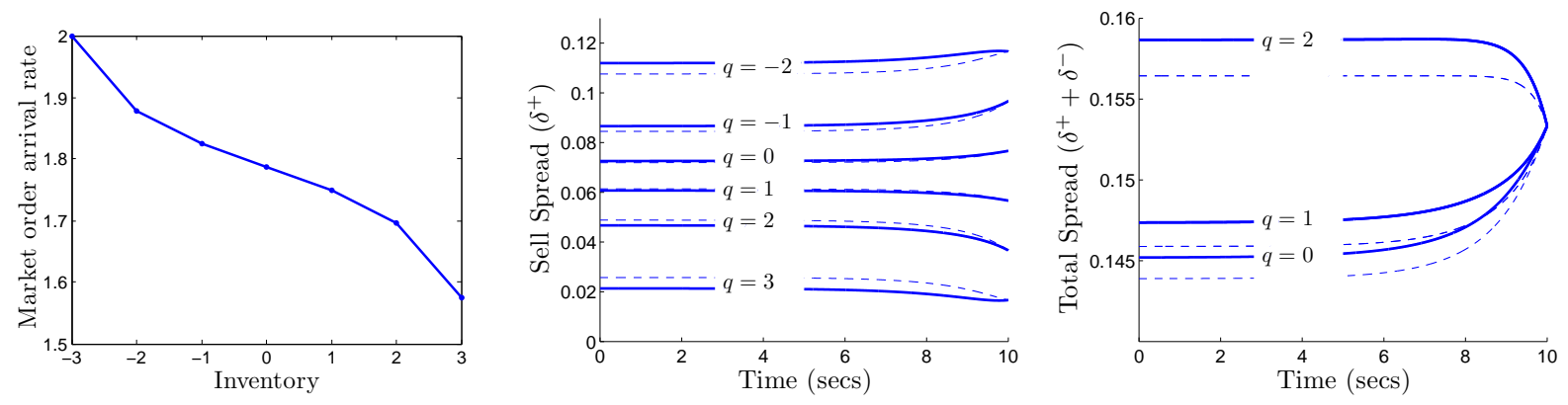

Figure 2.2: Effective market buy order arrival rate induced by optimal $g_{t}^{ \pm}$and optimal sell and total spreads for an MM who is ambiguity averse to MO rate of arrival (dashed lines are ambiguity neutral spreads). Parameter values are $\varphi_{\lambda}=6, \kappa^{ \pm}=15, \lambda^{ \pm}=2$, $\sigma=0.01, \alpha=0, \ell(q)=\theta q, \theta=0.01, \bar{q}=-\underline{q}=3$ and $T=10$.

the optimal $g_{t}^{ \pm}$are also shown in Figure 2.2. Note that when $q=\underline{q}$, the arrival rate of market buy orders does not change from that of the reference measure. This is because the MM is forced to post $\delta^{+}=\infty$ in this case, and so there is no change to the arrival rate that can have an effect on the MM's performance.

If the optimal spreads $\delta^{ \pm *}\left(t, q ; \varphi_{\lambda}\right)$ remain positive ${ }^{2}$, then substituting their feedback form into the expression for $g^{ \pm *}\left(t, q ; \varphi_{\lambda}\right)$ and into equation (2.9) give them simplified forms:

$$
g^{ \pm *}\left(t, q ; \varphi_{\lambda}\right)=-\frac{\varphi_{\lambda}}{\kappa^{ \pm}} e^{-\kappa^{ \pm}\left(\frac{1}{\kappa^{ \pm}}-h_{q-1}^{\varphi}+h_{q}^{\varphi}\right)}
$$

\footnotetext{
${ }^{2}$ Again, this condition can be violated, and there is no way of determining its validity without actually solving the equations.
} 
and

$$
\begin{aligned}
\partial_{t} h_{q}^{\varphi_{\lambda}}+\alpha q & +\frac{\lambda^{+}}{\varphi_{\lambda}}\left(1-e^{\left.-\frac{\varphi_{\lambda}}{\kappa^{+}} e^{-\kappa^{+}\left(\frac{1}{\kappa^{+}}-h_{q-1}^{\varphi}+h_{q}^{\varphi}\right)}\right)}\right) \mathbb{1}_{q \neq \underline{q}} \\
& +\frac{\lambda^{-}}{\varphi_{\lambda}}\left(1-e^{\left.-\frac{\varphi_{\lambda}}{\kappa^{-}} e^{-\kappa^{-}\left(\frac{1}{\kappa^{-}}-h_{q+1}^{\varphi_{\lambda}}+h_{q}^{\varphi} \lambda\right.}\right)}\right) \mathbb{1}_{q \neq \bar{q}}=0 .
\end{aligned}
$$

\subsubsection{Ambiguity aversion with respect to fill probability}

In a similar fashion to the previous section, the first step is to introduce a set of measure changes as follows:

$$
\left(\frac{d \mathbb{Q}(g)}{d \mathbb{P}}\right)=\prod_{i=+,-} \exp \left\{-\int_{0}^{T} \int_{0}^{\infty}\left(e^{g_{t}^{i}(y)}-1\right) \nu_{\mathbb{P}}^{i}(d y, d t)+\int_{0}^{T} \int_{0}^{\infty} g_{t}^{i}(y) \mu^{i}(d y, d t)\right\} .
$$

The form of the Radon-Nikodym derivative is the same as when only arrival rate is adjusted, except now the processes $g_{t}^{ \pm}(y)$ are allowed to depend on $y$. This allows the distribution of the maximum execution price to change. The full class of candidate measures to be considered by the MM is:

$$
\begin{array}{r}
\mathcal{Q}^{\kappa}=\left\{\mathbb{Q}(g): g^{ \pm}(y) \text { are } \mathcal{F}_{t}\right. \text {-predictable, Markov, and bounded, } \\
\text { and satisfy } \left.\int_{0}^{\infty} e^{g_{t}^{ \pm}(y)} F^{ \pm}(y) d y=1 \text { for all } t \in[0, T]\right\} .
\end{array}
$$

The integral constraint in the above definition ensures that it is only the distribution of maximum execution price that can change. The rate of market order arrivals in $\mathbb{Q}(g)$ will still be $\lambda^{ \pm}$. Let $\mathcal{G}^{ \pm}$be the corresponding class of functions which respect this integral constraint:

$$
\mathcal{G}^{ \pm}=\left\{g: \int_{0}^{\infty} e^{g(y)} F^{ \pm}(y) d y=1\right\}
$$


Denoting now the entropic penalization by $\mathcal{H}_{t, T}^{\varphi_{\kappa}}[\mathbb{Q}(g) \mid \mathbb{P}]$, it satisfies (see Section A.2 or Jacod and Shiryaev (1987)):

$$
\begin{aligned}
\mathbb{E}_{t, x, q, S}^{\mathbb{Q}(g)}\left[\mathcal{H}_{t, T}^{\varphi_{\kappa}}[\mathbb{Q}(g) \mid \mathbb{P}]\right] & =\frac{1}{\varphi_{\kappa}} \mathbb{E}_{t, x, q, S}^{\mathbb{Q}(g)}\left[\sum_{i=+,-} \int_{t}^{T} \int_{0}^{\infty}\left(e^{g_{u}^{i}(y)}\left(g_{u}^{i}(y)-1\right)+1\right) \nu_{\mathbb{P}}^{i}(d y, d u)\right] \\
& =\frac{1}{\varphi_{\kappa}} \mathbb{E}_{t, x, q, S}^{\mathbb{Q}(g)}\left[\sum_{i=+,-} \lambda^{i} \int_{t}^{T} \int_{0}^{\infty}\left(e^{g_{u}^{i}(y)}\left(g_{u}^{i}(y)-1\right)+1\right) F^{i}(y) d y d u\right] .
\end{aligned}
$$

and the infinitesimal generator $\mathcal{L}^{\mathbb{Q}(g)}$ is given by:

$$
\begin{array}{r}
\mathcal{L}^{\mathbb{Q}(g)} H^{\kappa}\left(t, x, q, S ; \varphi_{\kappa}\right)=\alpha \partial_{S} H^{\kappa}+\frac{1}{2} \sigma^{2} \partial_{S S} H^{\kappa} \\
+\lambda^{+} \int_{\delta^{+}}^{\infty} e^{g_{t}^{+}(y)} F^{+}(y) d y \mathcal{D}^{+} H^{\kappa} \mathbb{1}_{q \neq \underline{q}} \\
+\lambda^{-} \int_{\delta^{-}}^{\infty} e^{g_{t}^{-}(y)} F^{-}(y) d y \mathcal{D}^{-} H^{\kappa} \mathbb{1}_{q \neq \bar{q}}
\end{array}
$$

Making the usual ansatz $H^{\kappa}\left(t, x, q, S ; \varphi_{\kappa}\right)=x+q S+h_{q}^{\varphi_{\kappa}}(t)$ and substituting into equation (2.6), the function $h_{q}^{\varphi_{\kappa}}(t)$ now satisfies:

$$
\begin{aligned}
\partial_{t} h_{q}^{\varphi_{\kappa}}+\alpha q+\sup _{\delta^{+} \geq 0} \inf _{g^{+} \in \mathcal{G}^{+}}\left\{\lambda^{+}\left[\int_{\delta^{+}}^{\infty} e^{g^{+}(y)} F^{+}(y) d y\right]\left(\delta^{+}+h_{q-1}^{\varphi_{\kappa}}-h_{q}^{\varphi_{\kappa}}\right) \mathbb{1}_{q \neq \underline{q}}\right. & \\
\left.+\frac{1}{\varphi_{\kappa}} \lambda^{+} \int_{0}^{\infty}\left(e^{g^{+}(y)}\left(g^{+}(y)-1\right)+1\right) F^{+}(y) d y\right\} & \\
+\sup _{\delta^{-} \geq 0} \inf _{g^{-} \in \mathcal{G}^{-}}\left\{\lambda^{-}\left[\int_{\delta^{-}}^{\infty} e^{g^{-}(y)} F^{-}(y) d y\right]\left(\delta^{-}+h_{q+1}^{\varphi_{\kappa}}-h_{q}^{\varphi_{\kappa}}\right) \mathbb{1}_{q \neq \bar{q}}\right. & \\
\left.+\frac{1}{\varphi_{\kappa}} \lambda^{-} \int_{0}^{\infty}\left(e^{g^{-}(y)}\left(g^{-}(y)-1\right)+1\right) F^{-}(y) d y\right\} & =0, \\
h^{\varphi_{\kappa}}(T, q) & =-q \ell(q) .
\end{aligned}
$$

Proposition 3 (Optimal Feedback Controls). The optimal feedback controls of equation 
(2.11) are given by:

$$
\begin{aligned}
\delta^{+*}\left(t, q ; \varphi_{\kappa}\right) & =\left(\frac{1}{\varphi_{\kappa}} \log \left(1+\frac{\varphi_{\kappa}}{\kappa^{+}}\right)-h_{q-1}^{\varphi_{\kappa}}(t)+h_{q}^{\varphi_{\kappa}}(t)\right)_{+}, q \neq \underline{q}, \\
\delta^{-*}\left(t, q ; \varphi_{\kappa}\right)= & \left(\frac{1}{\varphi_{\kappa}} \log \left(1+\frac{\varphi_{\kappa}}{\kappa^{-}}\right)-h_{q+1}^{\varphi_{\kappa}}(t)+h_{q}^{\varphi_{\kappa}}(t)\right)_{+}, q \neq \bar{q}, \\
g^{ \pm *}\left(t, q, y ; \varphi_{\kappa}\right)= & -\log \left(1-e^{-\kappa^{ \pm} \delta^{ \pm *}\left(t, q ; \varphi_{\kappa}\right)}\left(1-e^{-\varphi_{\kappa}\left(\delta^{ \pm *}\left(t, q ; \varphi_{\kappa}\right)+h_{q \mp 1}^{\varphi_{\kappa}}(t)-h_{q}^{\varphi_{\kappa}}(t)\right)}\right)\right) \\
& -\varphi_{\kappa}\left(\delta^{ \pm *}\left(t, q ; \varphi_{\kappa}\right)+h_{q \mp 1}^{\varphi_{\kappa}}(t)-h_{q}^{\varphi_{\kappa}}(t)\right) \mathbb{1}_{y \geq \delta^{ \pm *}\left(t, q ; \varphi_{\kappa}\right)} .
\end{aligned}
$$

Proof. See Appendix B.1.

\section{Discussion and Financial Interpretation}

Note again that as $\varphi_{\kappa} \rightarrow 0$, the value of $g^{ \pm *}(y) \rightarrow 0$. Also note that in the optimal measure corresponding to $g^{ \pm *}(y)$ that the fill probability function is no longer exponential in $\delta^{ \pm}$. The new fill probability function can be written as:

$$
p_{t}\left(\delta^{ \pm}\right)=\int_{\delta^{ \pm}}^{\infty} e^{g^{ \pm *}\left(t, q, y ; \varphi^{\kappa}\right)} F^{ \pm}(y) d y .
$$

Due to the dependence of $g^{ \pm *}\left(t, q, y ; \varphi_{\kappa}\right)$ on the inventory level, the resulting fill probabilities now also depend on $q$. As $\delta^{ \pm} \rightarrow \infty, g^{ \pm *}(y) \rightarrow 0$ for all $y$, similar to the case of ambiguity on market order arrival rate. This happens for a similar reason: as the market maker posts very far away from the midprice, the probability of being filled is very small, and any change in distribution will have very little effect on what is already a very small quantity. On the other hand, unlike the case of ambiguity on market order arrival rate, as $\delta^{ \pm} \rightarrow 0, g^{ \pm *}(y) \rightarrow 0$. This happens because for any choice of maximum price distribution $e^{g^{ \pm}(y)} F^{ \pm}(y) d y$, posting at the midprice $\left(\delta^{ \pm}=0\right)$ guarantees that a market order will fill that posting. Thus, there is no choice of $g^{ \pm}(y)$ that can change the fill probability. Substituting the optimal $g^{ \pm *}\left(t, q, y ; \varphi_{\kappa}\right)$ into equation $(2.11)$ gives:

$$
\begin{aligned}
& \partial_{t} h_{q}^{\varphi_{\kappa}}+\alpha q+\sup _{\delta^{+} \geq 0}\left\{\frac{-\lambda^{+}}{\varphi_{\kappa}} \log \left(1-e^{-\kappa^{+} \delta^{+}}+e^{-\kappa^{+} \delta^{+}-\varphi_{\kappa}\left(\delta^{+}+h_{q-1}^{\varphi_{\kappa}}-h_{q}^{\varphi_{\kappa}}\right)}\right)\right\} \mathbb{1}_{q \neq \underline{q}} \\
& \sup _{\delta^{-} \geq 0}\left\{\frac{-\lambda^{-}}{\varphi_{\kappa}} \log \left(1-e^{-\kappa^{-} \delta^{-}}+e^{-\kappa^{-} \delta^{-}-\varphi_{\kappa}\left(\delta^{-}+h_{q+1}^{\varphi_{\kappa}}-h_{q}^{\varphi_{\kappa}}\right)}\right)\right\} \mathbb{1}_{q \neq \bar{q}}=0
\end{aligned}
$$


There is no known analytical solution to this equation, but a classical solution does exist (see Proposition 10). Solving this equation numerically allows the optimal spread and the effective fill probabilities to be shown in Figure 2.3. The fill probability functions
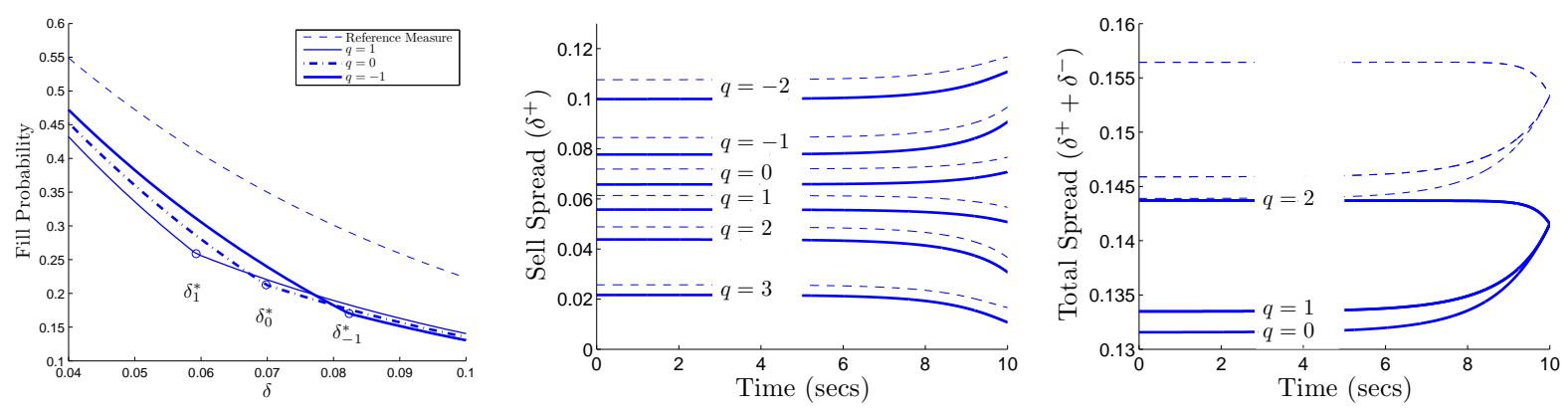

Figure 2.3: Typical fill probabilities of orders for various levels of $q$ and optimal sell and total spreads for an MM who is ambiguity averse to MO volume (dashed lines are ambiguity neutral spreads). Parameter values are $\varphi_{\kappa}=3, \kappa^{ \pm}=15, \lambda^{ \pm}=2, \sigma=0.01$, $\alpha=0, \ell(q)=\theta q, \theta=0.01, \bar{q}=-\underline{q}=3$

that result from $g^{ \pm *}$ indicate that the MM fears a smaller fill probability than dictated by the reference measure, and subsequently the rate at which their trades are filled is smaller than expected. To counteract the possibility that trades are not filled as fast as expected, the MM posts smaller spreads. Although the MM will earn less per trade with smaller spreads, this will have the effect of increasing the total number of trades that are made.

If the optimal spreads $\delta^{+*}\left(t, q ; \varphi_{\kappa}\right)$ remain positive, then their feedback form can be substituted into the expression for $g^{ \pm *}\left(t, q, y ; \varphi_{\kappa}\right)$ and equation (2.12) to give them simplified forms:

$g^{ \pm *}\left(t, q, y ; \varphi_{\kappa}\right)=-\log \left(1-\left(1+\frac{\kappa^{ \pm}}{\varphi_{\kappa}}\right)^{-1} e^{-\kappa^{ \pm} \delta^{ \pm *}\left(t, q ; \varphi_{\kappa}\right)}\right)-\log \left(1+\frac{\varphi_{\kappa}}{\kappa^{ \pm}}\right) \mathbb{1}_{y \geq \delta^{ \pm *}\left(t, q ; \varphi_{\kappa}\right)}$

and

$$
\begin{aligned}
\partial_{t} h_{q}^{\varphi_{\kappa}}+\alpha q & -\frac{\lambda^{+}}{\varphi_{\kappa}} \log \left(1-\left(1+\frac{\kappa^{+}}{\varphi_{\kappa}}\right)^{-1}\left(1+\frac{\varphi_{\kappa}}{\kappa^{+}}\right)^{-\frac{\kappa^{+}}{\varphi_{\kappa}}} e^{\kappa^{+}\left(h_{q-1}^{\varphi_{\kappa}}-h_{q}^{\varphi_{\kappa}}\right)}\right) \mathbb{1}_{q \neq \underline{q}} \\
- & \frac{\lambda^{-}}{\varphi_{\kappa}} \log \left(1-\left(1+\frac{\kappa^{-}}{\varphi_{\kappa}}\right)^{-1}\left(1+\frac{\varphi_{\kappa}}{\kappa^{-}}\right)^{-\frac{\kappa^{-}}{\varphi_{\kappa}}} e^{\kappa^{-}\left(h_{q+1}^{\varphi_{\kappa}}-h_{q}^{\varphi_{\kappa}}\right)}\right) \mathbb{1}_{q \neq \bar{q}}=0,
\end{aligned}
$$




\subsubsection{Ambiguity aversion with respect to midprice drift}

The equivalent measure changes used to incorporate ambiguity with respect to midprice drift are the following:

$$
\left(\frac{d \mathbb{Q}(\eta)}{d \mathbb{P}}\right)=\exp \left\{-\frac{1}{2} \int_{0}^{T}\left(\frac{\alpha-\eta_{t}}{\sigma}\right)^{2} d t-\int_{0}^{T} \frac{\alpha-\eta_{t}}{\sigma} d W_{t}\right\}
$$

In the measure $\mathbb{Q}(\eta)$, the drift of the asset's midprice changes from $\alpha$ to $\eta_{t}$. The full class of measures to be considered by the MM is:

$$
\mathcal{Q}^{\alpha}=\left\{\mathbb{Q}(\eta): \eta \text { is } \mathcal{F}_{t} \text {-predictable, Markov, and bounded }\right\} \text {. }
$$

With this choice of equivalent measures and denoting the entropic penalty function by $\mathcal{H}_{t, T}^{\varphi_{\alpha}}[\mathbb{Q}(\eta) \mid \mathbb{P}]$, it is seen to satisfy:

$$
\mathbb{E}_{t, x, q, S}^{\mathbb{Q}(\eta)}\left[\mathcal{H}_{t, T}^{\varphi_{\alpha}}[\mathbb{Q}(\eta) \mid \mathbb{P}]\right]=\mathbb{E}_{t, x, q, S}^{\mathbb{Q}(\eta)}\left[\frac{1}{2 \varphi_{\alpha}} \int_{t}^{T}\left(\frac{\alpha-\eta_{u}}{\sigma}\right)^{2} d u\right]
$$

and the infinitesimal generator $\mathcal{L}^{\mathbb{Q}(\eta)}$ is given by:

$$
\begin{aligned}
\mathcal{L}^{\mathbb{Q}(\eta)} H^{\alpha}\left(t, x, q, S ; \varphi_{\alpha}\right)= & \eta_{t} \partial_{S} H^{\alpha}+\frac{1}{2} \sigma^{2} \partial_{S S} H^{\alpha} \\
& +\lambda^{+} e^{-\kappa^{+} \delta^{+}} \mathcal{D}^{+} H^{\alpha} \mathbb{1}_{q \neq \underline{q}}+\lambda^{-} e^{-\kappa^{-} \delta^{-}} \mathcal{D}^{-} H^{\alpha} \mathbb{1}_{q \neq \bar{q}}
\end{aligned}
$$

Making the ansatz $H^{\alpha}\left(t, x, q, S ; \varphi_{\alpha}\right)=x+q S+h_{q}^{\varphi_{\alpha}}(t)$, function $h_{q}^{\varphi_{\alpha}}(t)$ satisfies:

$$
\begin{aligned}
\partial_{t} h_{q}^{\varphi_{\alpha}}+\sup _{\delta^{ \pm} \geq 0} \inf _{\eta}\left\{\lambda^{+} e^{-\kappa^{+} \delta^{+}}\left(\delta^{+}+h_{q-1}^{\varphi_{\alpha}}-h_{q}^{\varphi_{\alpha}}\right) \mathbb{1}_{q \neq \underline{q}}\right. \\
+\lambda^{-} e^{-\kappa^{-} \delta^{-}}\left(\delta^{-}+h_{q+1}^{\varphi_{\alpha}}-h_{q}^{\varphi_{\alpha}}\right) \mathbb{1}_{q \neq \bar{q}} \\
\left.+\eta q+\frac{1}{2 \varphi_{\alpha}}\left(\frac{\alpha-\eta}{\sigma}\right)^{2}\right\}=0 \\
h_{q}^{\varphi_{\alpha}}(T)=-q(2.13)
\end{aligned}
$$

Proposition 4 (Optimal Feedback Controls). The optimal feedback controls of equation 
(2.13) are given by

$$
\begin{aligned}
\delta^{+*}\left(t, q ; \varphi_{\alpha}\right) & =\left(\frac{1}{\kappa^{+}}-h_{q-1}^{\varphi_{\alpha}}(t)+h_{q}^{\varphi_{\alpha}}(t)\right)_{+}, q \neq \underline{q} \\
\delta^{-*}\left(t, q ; \varphi_{\alpha}\right) & =\left(\frac{1}{\kappa^{-}}-h_{q+1}^{\varphi_{\alpha}}(t)+h_{q}^{\varphi_{\alpha}}(t)\right)_{+}, q \neq \bar{q} \\
\eta^{*}\left(t, q ; \varphi_{\alpha}\right) & =\alpha-\varphi_{\alpha} \sigma^{2} q .
\end{aligned}
$$

Proof. The optimal $\delta^{ \pm}$is solved for in an equivalent manner to Proposition 1. For the optimal $\eta$, apply first order conditions in $\eta$ :

$$
\begin{aligned}
0 & =q-\frac{1}{\varphi_{\alpha}} \frac{\alpha-\eta}{\sigma} \frac{1}{\sigma} \\
\varphi_{\alpha} q \sigma^{2} & =-(\alpha-\eta) \\
\eta & =\alpha-\varphi_{\alpha} \sigma^{2} q
\end{aligned}
$$

as desired.

\section{Discussion and Financial Interpretation}

Substituting the optimal $\eta^{*}\left(t, q ; \varphi_{\alpha}\right)$ into equation (2.13) gives:

$$
\begin{aligned}
\partial_{t} h_{q}^{\varphi_{\alpha}}+\alpha q-\frac{1}{2} \varphi_{\alpha} \sigma^{2} q^{2} & +\sup _{\delta^{+} \geq 0}\left\{\lambda^{+} e^{-\kappa^{+} \delta^{+}}\left(\delta^{+}+h_{q-1}^{\varphi_{\alpha}}-h_{q}^{\varphi_{\alpha}}\right)\right\} \mathbb{1}_{q \neq \underline{q}} \\
& +\sup _{\delta^{-} \geq 0}\left\{\lambda^{-} e^{-\kappa^{-} \delta^{-}}\left(\delta^{-}+h_{q+1}^{\varphi_{\alpha}}-h_{q}^{\varphi_{\alpha}}\right)\right\} \mathbb{1}_{q \neq \bar{q}}=0
\end{aligned}
$$

This equation has a classical solution (see Proposition 10), and under certain circumstances it has a closed form analytical solution (discussed in Section (2.4)), but otherwise it is solved numerically. As long as the spreads $\delta^{* \pm}\left(t, q ; \varphi_{\alpha}\right)$ remain positive, substituting their feedback expressions into equation (2.14) gives:

$$
\begin{aligned}
\partial_{t} h_{q}^{\varphi_{\alpha}}+\alpha q-\frac{1}{2} \varphi_{\alpha} \sigma^{2} q^{2} & +\frac{\lambda^{+}}{\kappa^{+}} e^{-\kappa^{+}\left(\frac{1}{\kappa^{+}}-h_{q-1}^{\varphi_{\alpha}}+h_{q}^{\varphi_{\alpha}}\right)} \mathbb{1}_{q \neq \underline{q}} \\
+ & \left.\frac{\lambda^{-}}{\kappa^{-}} e^{-\kappa^{-}\left(\frac{1}{\kappa^{-}}-h_{q+1}^{\varphi_{\alpha}}+h_{q}^{\varphi_{\alpha}}\right)}\right)_{\mathbb{1}_{q \neq \bar{q}}}=0
\end{aligned}
$$

The relationship between inventory and the midprice drift in the optimal measure should 
make sense when one interprets the new drift as the state of the world that the MM fears to be real. When the MM holds positive inventory, it is undesirable for the midprice drift to be less than that of the reference measure, and this would induce losses for the MM. As can be seen in Figure 2.4, the MM posts spreads in such a fashion to induce faster mean reversion to zero inventory as compared to the reference model.
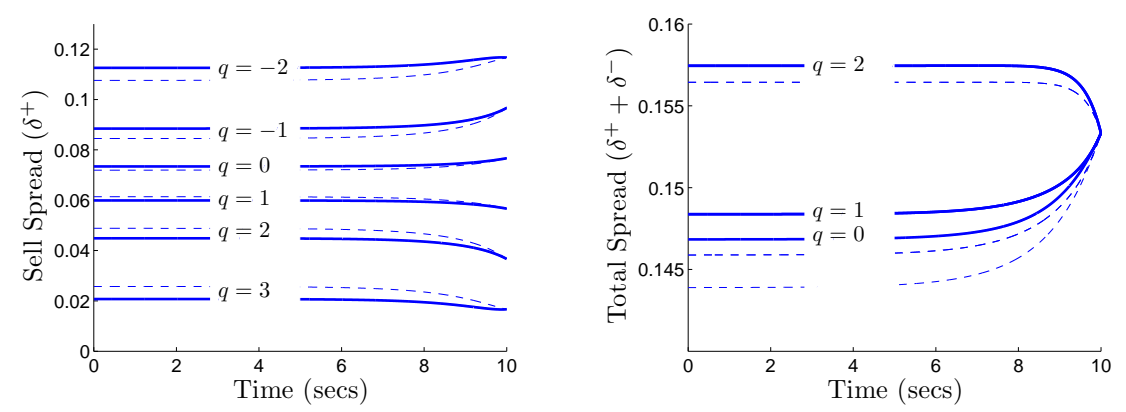

Figure 2.4: Optimal sell and total spreads for an MM who is ambiguity averse to midprice drift (dashed lines are ambiguity neutral spreads). Parameter values are $\varphi_{\alpha}=20, \kappa^{ \pm}=$ $15, \lambda^{ \pm}=2, \sigma=0.01, \alpha=0, \ell(q)=\theta q, \theta=0.01, \bar{q}=-\underline{q}=3$ and $T=10$ seconds.

\section{Equivalence to Inventory Penalization}

Consider a MM that is not ambiguity averse, and so they act with full confidence under the reference model. Suppose that they introduce an additional inventory risk control by incorporating a penalty for holding non-zero inventory positions over time in the following manner:

$$
H^{\phi}(t, x, q, S)=\sup _{\left(\delta_{u}^{ \pm}\right)_{t \leq u \leq T} \in \mathcal{A}} \mathbb{E}_{t, x, q, S}^{\mathbb{P}}\left[X_{T}+q_{T}\left(S_{T}-\ell\left(q_{T}\right)\right)-\frac{1}{2} \phi \sigma^{2} \int_{t}^{T} q_{u}^{2} d u\right],
$$

The penalty term arises as the variance of the market maker's inventory value, assuming a mark-to-market value at the midprice. See Cartea and Jaimungal (2012) for more details on this type of penalty. After the usual ansatz, substitution into the corresponding HJB 
equation, and solving for the optimal controls $\delta^{ \pm}$, the resulting system of ODE's is:

$$
\begin{aligned}
\partial_{t} h_{q}^{\phi}+\alpha q-\frac{1}{2} \phi \sigma^{2} q^{2} & +\sup _{\delta^{+} \geq 0}\left\{\lambda^{+} e^{-\kappa^{+} \delta^{+}}\left(\delta^{+}+h_{q-1}^{\phi}-h_{q}^{\phi}\right)\right\} \mathbb{1}_{q \neq \underline{q}} \\
& +\sup _{\delta^{-} \geq 0}\left\{\lambda^{-} e^{-\kappa^{-} \delta^{-}}\left(\delta^{-}+h_{q+1}^{\phi}-h_{q}^{\phi}\right)\right\} \mathbb{1}_{q \neq \bar{q}}=0
\end{aligned}
$$

This equation is seen to be equivalent to equation (2.14) with $\varphi_{\alpha}=\phi$. The fact that the two differential equations for the value functions are the same means that the value functions themselves are the same:

$$
\begin{array}{r}
\sup _{\left(\delta_{u}^{ \pm}\right)_{t \leq u \leq T} \in \mathcal{A}} \mathbb{E}_{t, x, q, S}^{\mathbb{P}}\left[X_{T}+q_{T}\left(S_{T}-\ell\left(q_{T}\right)\right)-\frac{1}{2} \phi \sigma^{2} \int_{t}^{T} q_{u}^{2} d u\right] \\
=\sup _{\left(\delta_{u}^{ \pm}\right)_{t \leq u \leq T} \in \mathcal{A}} \inf _{\mathbb{Q} \in \mathcal{Q}^{\alpha}} \mathbb{E}_{t, x, q, S}^{\mathbb{Q}}\left[X_{T}+q_{T}\left(S_{T}-\ell\left(q_{T}\right)\right)+\mathcal{H}_{t, T}[\mathbb{Q} \mid \mathbb{P}]\right]
\end{array}
$$

In effect, a MM which is fully confident in their model but incorporates a running quadratic inventory penalty behaves in an equivalent manner to a MM that is adverse to misspecification to the drift of the asset's midprice.

From an intuitive perspective of what a MM would do, it makes sense that ambiguity on drift has the same effects as a cumulative inventory penalization. From the resulting form of $\eta^{*}$, it is seen that the market maker fears that the drift is in a direction that lowers the value of their inventory holdings over time, thus holding inventory suffers the ambiguity penalty and the market maker adjusts their posting levels $\delta^{ \pm}$so that inventory typically remains closer to zero than usual. This is the same type of change in posting levels that would be expected from an ambiguity neutral market maker that imposes a cumulative inventory penalty; holding large quantities of inventory incurs a larger penalty, and so posting levels are adjusted so that inventory generally remains closer to zero.

Many optimal trading problems incorporate this type of cumulative penalty due to its ability to control the risk levels achieved by the market maker, much in the same way as imposing a maximum and minimum inventory level. The equivalence demonstrated here illustrate what is possibly a more fundamental reason for imposing a cumulative penalty. This will have two important future consequences: first, if the asset's dynamics do not lend themselves to a natural cumulative penalty of the form $\frac{1}{2} \phi \sigma^{2} \int_{0}^{T} q_{t}^{2} d t$, then the correct form of inventory penalization should be deduced from the results of ambiguity analysis when performed on the dynamics of the midprice. Second, this equivalence raises the question as to whether other types of risk controls that are imposed have an 
interpretation that they instead result from the agent's level of ambiguity aversion to some factor of the model. An example of such an interpretation will be given again in Sections 5.2 and 5.4 .

\subsection{Mixed Ambiguity}

Up to this point, the MM has been able to account for ambiguity with respect to three factors of the reference model separately. However, the MM would ideally like to account for ambiguity with respect to different factors of the model simultaneously, possibly with different levels of ambiguity on each factor. In this section, a method which simultaneously incorporates all three types of ambiguity is developed.

The key ingredients to this method will be in properly defining the measure changes and the associated penalty function. The main idea is to think of the measure change as a sequence of three measure changes, each one corresponding to a change in one of the model factors. Each of these measure changes will be assigned a weight corresponding to the MM's level of ambiguity towards that model factor. Issues of concern with this method are whether the order of the three measure changes has an effect on the value of the penalty function, and whether the associated penalty function behaves as expected with desired properties. The desired properties of the penalty are as follows:

1. The penalty term has positive expectation under the candidate measure.

2. Suppose the MM has ambiguity levels $\varphi_{\lambda}, \varphi_{\kappa}$, and $\varphi_{\alpha}$. As any two of these parameters approach zero, the resulting penalty should result in a value function and optimal controls that are equal to the previously discussed value function and optimal controls corresponding to the non-zero parameter.

Let $\mathbb{Q}^{\alpha, \lambda . \kappa}(\eta, g)$ denote a candidate measure in which the midprice drift, MO arrival rate, and maximum execution price distribution have all changed. Specifically, define 
$\mathbb{Q}^{\alpha, \lambda . \kappa}(\eta, g)$ by the following Radon-Nikodym derivative:

$$
\begin{aligned}
\left(\frac{d \mathbb{Q}^{\alpha, \lambda . \kappa}(\eta, g)}{d \mathbb{P}}\right)= & \exp \left\{-\frac{1}{2} \int_{0}^{T}\left(\frac{\alpha-\eta_{t}}{\sigma}\right)^{2} d t-\int_{0}^{T} \frac{\alpha-\eta_{t}}{\sigma} d W_{t}\right\} \times \\
& \prod_{i=+,-} \exp \left\{-\int_{0}^{T} \int_{0}^{\infty}\left(e^{g_{t}^{i}(y)}-1\right) \nu_{\mathbb{P}}^{i}(d y, d t)+\int_{0}^{T} \int_{0}^{\infty} g_{t}^{i}(y) \mu^{i}(d y, d t)\right\}
\end{aligned}
$$

In this measure, the drift of the midprice will be $\eta_{t}$ and the compensator of $\mu^{ \pm}$will be $e^{g_{t}^{ \pm}(y)} \nu_{\mathbb{P}}^{ \pm}$. This single measure change can be thought of as three measure changes occuring in sequence, each one changing only one factor of the model at a time. Two natural choices for the sequence of measure changes are shown in Figure 2.5. In changing from

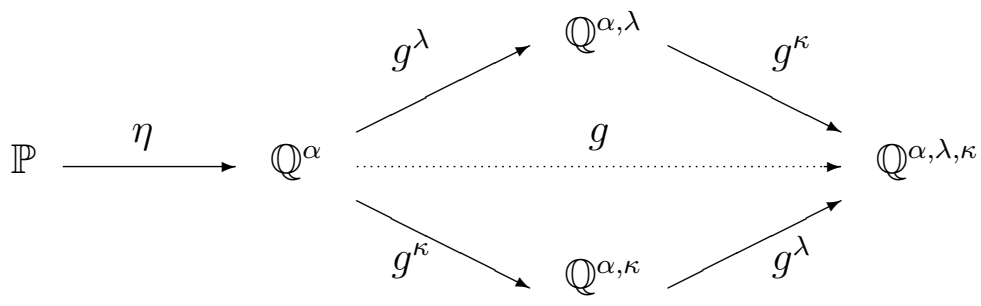

Figure 2.5: The two natural alternative routes from the reference measure $\mathbb{P}$ to a candidate measure $\mathbb{Q}^{\alpha, \lambda, \kappa}$ in which midprice drift, MO intensity, and MO execution price have been altered.

$\mathbb{Q}^{\alpha}(\eta)$ to $\mathbb{Q}^{\alpha, \lambda, \kappa}(\eta, g)$, the new MO intensity and maximum execution price will be fully characterized by processes $g_{t}^{ \pm}(y)$ appearing in equation (2.17). The appropriate processes which correspond to the intermediate measure changes are given by the following:

Proposition 5 (Intermediate measure changes). Let $\mathbb{Q}^{\alpha}(\eta)$ be a measure that has the same $M O$ intensity and maximum execution price distribution as $\mathbb{P}$. Let $\mathbb{Q}^{\alpha, \lambda, \kappa}(\eta, g)$ be given by:

$$
\frac{d \mathbb{Q}^{\alpha, \lambda, \kappa}(\eta, g)}{d \mathbb{Q}^{\alpha}(\eta)}=\prod_{i=+,-} \exp \left\{-\int_{0}^{T} \int_{0}^{\infty}\left(e^{g_{t}^{i}(y)}-1\right) \nu_{\mathbb{P}}^{i}(d y, d t)+\int_{0}^{T} \int_{0}^{\infty} g_{t}^{i}(y) \mu^{i}(d y, d t)\right\}
$$

Then the desired processes that define measures $\mathbb{Q}^{\alpha, \lambda}\left(\eta, g^{\lambda}\right)$ and $\mathbb{Q}^{\alpha, \kappa}\left(\eta, g^{\kappa}\right)$ in Figure 2.5 
are given by:

$$
g_{t}^{ \pm \lambda}=\log \int_{0}^{\infty} e^{g_{t}^{ \pm}(y)} F^{ \pm}(y) d y \quad \text { and } \quad g_{t}^{ \pm \kappa}(y)=g_{t}^{ \pm}(y)-g_{t}^{ \pm \lambda} .
$$

Moreover, this diagram commutes.

Proof. Given the measure $\mathbb{Q}^{\alpha, \lambda, \kappa}(\eta, g)$ which arises from using the process $g_{t}^{ \pm}(y)$ in equation (2.18), the effective rate of market order arrivals in this measure is:

$$
\lambda_{t}^{ \pm}\left(g^{ \pm}\right)=\lambda^{ \pm} \int_{0}^{\infty} e^{g_{t}^{ \pm}(y)} F^{ \pm}(y) d y
$$

In order to change measure from $\mathbb{Q}^{\alpha}(\eta)$ to $\mathbb{Q}^{\alpha, \lambda}\left(\eta, g^{\lambda}\right)$ where only the effective rate of market order arrivals has changed, and this rate is now equal to $\lambda_{t}^{ \pm}\left(g_{t}^{ \pm}\right)$, a RadonNikodym derivative process of the form in equation (2.7) is used:

$$
\left(\frac{d \mathbb{Q}\left(\eta, g^{\lambda}\right)}{d \mathbb{Q}(\eta)}\right)=\prod_{i=+,-} \exp \left\{-\int_{0}^{T} \int_{0}^{\infty}\left(e^{g_{t}^{i \lambda}}-1\right) \nu_{\mathbb{P}}^{i}(d y, d t)+\int_{0}^{T} \int_{0}^{\infty} g_{t}^{i \lambda} \mu^{i}(d y, d t)\right\},
$$

As stated in Section 2.2.1 and also outlined in Section A.2, this measure change results in effective rates of $e^{g_{t}^{ \pm \lambda}} \lambda^{ \pm}$. Clearly, $g_{t}^{ \pm \lambda}$ must be chosen so that $e^{g_{t}^{ \pm \lambda}} \lambda^{ \pm}=\lambda^{ \pm} \int_{0}^{\infty} e^{g_{t}^{ \pm}(y)} F^{ \pm}(y) d y$, or $g_{t}^{ \pm \lambda}=\log \int_{0}^{\infty} e^{g_{t}^{ \pm}(y)} F^{ \pm}(y) d y$.

Next, define $g_{t}^{ \pm \kappa}(y)=g_{t}^{ \pm}(y)-g_{t}^{ \pm \lambda}$, and use this process to define a new measure through that Radon-Nikodym derivative process:

$$
\left(\frac{d \mathbb{Q}\left(\eta, g^{\kappa}\right)}{d \mathbb{Q}(\eta)}\right)=\prod_{i=+,-} \exp \left\{-\int_{0}^{T} \int_{0}^{\infty}\left(e^{g_{t}^{i \kappa}(y)}-1\right) \nu_{\mathbb{P}}^{i}(d y, d t)+\int_{0}^{T} \int_{0}^{\infty} g_{t}^{i \kappa}(y) \mu^{i}(d y, d t)\right\}
$$

It must be shown now that the effective rate of market orders in this measure is $\lambda^{ \pm}$(so 
that only the distribution of their maximum execution price has changed).

$$
\begin{aligned}
\lambda_{t}^{ \pm}\left(g^{\kappa}\right) & =\lambda^{ \pm} \int_{0}^{\infty} e^{g_{t}^{ \pm \kappa}(y)} F^{ \pm}(y) d y \\
& =\lambda^{ \pm} \int_{0}^{\infty} e^{g_{t}^{ \pm}(y)-g_{t}^{ \pm \lambda}} F^{ \pm}(y) d y \\
& =e^{-g_{t}^{ \pm \lambda}} \lambda^{ \pm} \int_{0}^{\infty} e^{g_{t}^{ \pm}(y)} F^{ \pm}(y) d y \\
& =\frac{1}{\int_{0}^{\infty} e^{g_{t}^{ \pm}(y)} F^{ \pm}(y) d y} \lambda^{ \pm} \int_{0}^{\infty} e^{g_{t}^{ \pm}(y)} F^{ \pm}(y) d y \\
& =\lambda^{ \pm}
\end{aligned}
$$

as desired. Commutativity of Figure 2.5 follows from the additive relation between $g^{ \pm \lambda}$, $g^{ \pm \kappa}$ and $g^{ \pm}$.

The total measure change can be decomposed as either of the two following products, depending on which of the two routes is used:

$$
\begin{aligned}
& \frac{d \mathbb{Q}^{\alpha, \lambda, \kappa}(\eta, g)}{d \mathbb{P}}=\frac{d \mathbb{Q}^{\alpha}(\eta)}{d \mathbb{P}} \frac{d \mathbb{Q}^{\alpha, \lambda}\left(\eta, g^{\lambda}\right)}{d \mathbb{Q}^{\alpha}(\eta)} \frac{d \mathbb{Q}^{\alpha, \lambda, \kappa}(\eta, g)}{d \mathbb{Q}^{\alpha, \lambda}\left(\eta, g^{\lambda}\right)} \\
& \frac{d \mathbb{Q}^{\alpha, \lambda, \kappa}(\eta, g)}{d \mathbb{P}}=\frac{d \mathbb{Q}^{\alpha}(\eta)}{d \mathbb{P}} \frac{d \mathbb{Q}^{\alpha, \kappa}\left(\eta, g^{\kappa}\right)}{d \mathbb{Q}^{\alpha}(\eta)} \frac{d \mathbb{Q}^{\alpha, \lambda, \kappa}(\eta, g)}{d \mathbb{Q}^{\alpha, \kappa}\left(\eta, g^{\kappa}\right)}
\end{aligned}
$$

Applying a logarithm and assigning ambiguity weights to each term allows one to consider two possibilities for the penalty function:

$$
\begin{gathered}
\frac{1}{\varphi_{\alpha}} \log \left(\frac{d \mathbb{Q}^{\alpha}(\eta)}{d \mathbb{P}}\right)+\frac{1}{\varphi_{\lambda}} \log \left(\frac{d \mathbb{Q}^{\alpha, \lambda}\left(\eta, g^{\lambda}\right)}{d \mathbb{Q}^{\alpha}(\eta)}\right)+\frac{1}{\varphi_{\kappa}} \log \left(\frac{d \mathbb{Q}^{\alpha, \lambda, \kappa}(\eta, g)}{d \mathbb{Q}^{\alpha, \lambda}\left(\eta, g^{\lambda}\right)}\right) \\
\frac{1}{\varphi_{\alpha}} \log \left(\frac{d \mathbb{Q}^{\alpha}(\eta)}{d \mathbb{P}}\right)+\frac{1}{\varphi_{\kappa}} \log \left(\frac{d \mathbb{Q}^{\alpha, \kappa}\left(\eta, g^{\kappa}\right)}{d \mathbb{Q}^{\alpha}(\eta)}\right)+\frac{1}{\varphi_{\lambda}} \log \left(\frac{d \mathbb{Q}^{\alpha, \lambda, \kappa}(\eta, g)}{d \mathbb{Q}^{\alpha, \kappa}\left(\eta, g^{\kappa}\right)}\right)
\end{gathered}
$$

Schematically, these two expressions correspond to the association of ambiguity weights as in Figure 2.6. When the expressions in equations (2.19) and (2.20) are considered under the expectation in the candidate measure $\mathbb{Q}^{\alpha, \lambda, \kappa}(\eta, g)$, only one of them can be guaranteed to be positive. This dictates that the appropriate choice of penalty function is the following:

$$
\mathcal{H}\left(\mathbb{Q}^{\alpha, \lambda, \kappa}(\eta, g) \mid \mathbb{P}\right)=\mathcal{H}^{\alpha}\left(\mathbb{Q}^{\alpha}(\eta) \mid \mathbb{P}\right)+\mathcal{H}^{\lambda, \kappa}\left(\mathbb{Q}^{\alpha, \lambda, \kappa}(\eta, g) \mid \mathbb{Q}^{\alpha}(\eta)\right)
$$




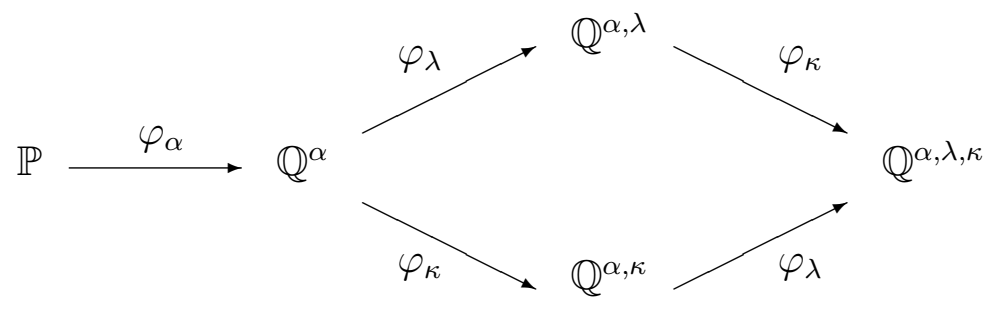

Figure 2.6: The two natural alternative routes from the reference measure $\mathbb{P}$ to a candidate measure $\mathbb{Q}^{\alpha, \lambda, \kappa}$, and the associated levels of ambiguity for each changing factor.

where:

$\mathcal{H}^{\lambda, \kappa}\left(\mathbb{Q}^{\alpha, \lambda, \kappa}(\eta, g) \mid \mathbb{Q}^{\alpha}(\eta)\right)= \begin{cases}\frac{1}{\varphi_{\lambda}} \log \left(\frac{d \mathbb{Q}^{\alpha, \lambda}\left(\eta, g^{\lambda}\right)}{d \mathbb{Q}^{\alpha}(\eta)}\right)+\frac{1}{\varphi_{\kappa}} \log \left(\frac{d \mathbb{Q}^{\alpha, \lambda, \kappa}(\eta, g)}{d \mathbb{Q}^{\alpha, \lambda}\left(\eta, g^{\lambda}\right)}\right), & \text { if } \varphi_{\lambda} \geq \varphi_{\kappa} \\ \frac{1}{\varphi_{\kappa}} \log \left(\frac{d \mathbb{Q}^{\alpha, \kappa}\left(\eta, g^{\kappa}\right)}{d \mathbb{Q}^{\alpha}(\eta)}\right)+\frac{1}{\varphi_{\lambda}} \log \left(\frac{d \mathbb{Q}^{\alpha, \lambda, \kappa}(\eta, g)}{d \mathbb{Q}^{\alpha, \kappa}\left(\eta, g^{\kappa}\right)}\right), & \text { if } \varphi_{\lambda} \leq \varphi_{\kappa}\end{cases}$

Note that when $\varphi_{\lambda}=\varphi_{\kappa}$ the two expressions for $\mathcal{H}^{\lambda, \kappa}\left(\mathbb{Q}^{\alpha, \lambda, \kappa}(\eta, g) \mid \mathbb{Q}^{\alpha}(\eta)\right)$ in $(2.22)$ agree. This will correspond to a special case to be discussed in Section (2.4).

Proposition 6 (Non-negativity of mixed penalty function). The penalty function defined by equations (2.21) and (2.22) satisfies:

$$
\mathbb{E}^{\mathbb{Q}^{\alpha, \lambda, \kappa}(\eta, g)}\left[\mathcal{H}\left(\mathbb{Q}^{\alpha, \lambda, \kappa}(\eta, g) \mid \mathbb{P}\right)\right] \geq 0
$$

Proof. Suppose $\varphi_{\lambda}>\varphi_{\kappa}$. Then the penalty function is:

$\mathcal{H}\left(\mathbb{Q}^{\alpha, \lambda, \kappa}(\eta, g) \mid \mathbb{P}\right)=\frac{1}{\varphi_{\alpha}} \log \left(\frac{d \mathbb{Q}^{\alpha}(\eta)}{d \mathbb{P}}\right)+\frac{1}{\varphi_{\lambda}} \log \left(\frac{d \mathbb{Q}^{\alpha, \lambda}\left(\eta, g^{\lambda}\right)}{d \mathbb{Q}^{\alpha}(\eta)}\right)+\frac{1}{\varphi_{\kappa}} \log \left(\frac{d \mathbb{Q}^{\alpha, \lambda, \kappa}(\eta, g)}{d \mathbb{Q}^{\alpha, \lambda}\left(\eta, g^{\lambda}\right)}\right)$ 
The expectation $\mathbb{E}^{\mathbb{Q}^{\alpha, \lambda, \kappa}(\eta, g)}\left[\mathcal{H}\left(\mathbb{Q}^{\alpha, \lambda, \kappa}(\eta, g) \mid \mathbb{P}\right)\right]$ is taken in two parts. First, compute:

$$
\begin{aligned}
& \mathbb{E}^{\mathbb{Q}^{\alpha, \lambda, \kappa}(\eta, g)}\left[\frac{1}{\varphi_{\alpha}} \log \left(\frac{d \mathbb{Q}^{\alpha}(\eta)}{d \mathbb{P}}\right)\right] \\
= & \frac{1}{\varphi_{\alpha}} \mathbb{E}^{\mathbb{P}}\left[\frac{d \mathbb{Q}^{\alpha, \lambda, \kappa}(\eta, g)}{d \mathbb{P}} \log \left(\frac{d \mathbb{Q}^{\alpha}(\eta)}{d \mathbb{P}}\right)\right] \\
= & \frac{1}{\varphi_{\alpha}} \mathbb{E}^{\mathbb{P}}\left[\frac{d \mathbb{Q}^{\alpha, \lambda, \kappa}(\eta, g)}{d \mathbb{Q}^{\alpha}(\eta)} \frac{d \mathbb{Q}^{\alpha}(\eta)}{d \mathbb{P}} \log \left(\frac{d \mathbb{Q}^{\alpha}(\eta)}{d \mathbb{P}}\right)\right] \\
= & \frac{1}{\varphi_{\alpha}} \mathbb{E}^{\mathbb{P}}\left[\frac{d \mathbb{Q}^{\alpha, \lambda, \kappa}(\eta, g)}{d \mathbb{Q}^{\alpha}(\eta)}\right] \mathbb{E}^{\mathbb{P}}\left[\frac{d \mathbb{Q}^{\alpha}(\eta)}{d \mathbb{P}} \log \left(\frac{d \mathbb{Q}^{\alpha}(\eta)}{d \mathbb{P}}\right)\right] \\
= & \frac{1}{\varphi_{\alpha}} \mathbb{E}^{\mathbb{P}}\left[\frac{d \mathbb{Q}^{\alpha}(\eta)}{d \mathbb{P}} \log \left(\frac{d \mathbb{Q}^{\alpha}(\eta)}{d \mathbb{P}}\right)\right] \\
\geq & 0
\end{aligned}
$$

The expectation can be factored because the Radon-Nikodym derivative $\frac{d \mathbb{Q}^{\alpha, \lambda, \kappa}(\eta, g)}{d \mathbb{Q}^{\alpha}(\eta)}$ is generated by the Poisson random measures, and $\frac{d \mathbb{Q}^{\alpha}(\eta)}{d \mathbb{P}}$ is generated by the Brownian motion, which are independent from each other. That means these terms have zero cross-variation. The second computation to consider is:

$$
\begin{aligned}
& \mathbb{E}^{\mathbb{Q}^{\alpha, \lambda, \kappa}(\eta, g)}\left[\frac{1}{\varphi_{\lambda}} \log \left(\frac{d \mathbb{Q}^{\alpha, \lambda}\left(\eta, g^{\lambda}\right)}{d \mathbb{Q}^{\alpha}(\eta)}\right)+\frac{1}{\varphi_{\kappa}} \log \left(\frac{d \mathbb{Q}^{\alpha, \lambda, \kappa}(\eta, g)}{d \mathbb{Q}^{\alpha, \lambda}\left(\eta, g^{\lambda}\right)}\right)\right] \\
= & \frac{1}{\varphi_{\lambda}} \mathbb{E}^{\mathbb{Q}^{\alpha, \lambda, \kappa}(\eta, g)}\left[\log \left(\frac{d \mathbb{Q}^{\alpha, \lambda}\left(\eta, g^{\lambda}\right)}{d \mathbb{Q}^{\alpha}(\eta)}\right)\right]+\frac{1}{\varphi_{\kappa}} \mathbb{E}^{\mathbb{Q}^{\alpha, \lambda, \kappa}(\eta, g)}\left[\log \left(\frac{d \mathbb{Q}^{\alpha, \lambda, \kappa}(\eta, g)}{d \mathbb{Q}^{\alpha, \lambda}\left(\eta, g^{\lambda}\right)}\right)\right] \\
= & \frac{1}{\varphi_{\lambda}}\left(\mathbb{E}^{\mathbb{Q}^{\alpha, \lambda, \kappa}(\eta, g)}\left[\log \left(\frac{d \mathbb{Q}^{\alpha, \lambda}\left(\eta, g^{\lambda}\right)}{d \mathbb{Q}^{\alpha}(\eta)}\right)\right]+\frac{\varphi_{\lambda}}{\varphi_{\kappa}} \mathbb{E}^{\mathbb{Q}^{\alpha, \lambda, \kappa}(\eta, g)}\left[\log \left(\frac{d \mathbb{Q}^{\alpha, \lambda, \kappa}(\eta, g)}{d \mathbb{Q}^{\alpha, \lambda}\left(\eta, g^{\lambda}\right)}\right)\right]\right) \\
\geq & \frac{1}{\varphi_{\lambda}}\left(\mathbb{E}^{\mathbb{Q}^{\alpha, \lambda, \kappa}(\eta, g)}\left[\log \left(\frac{d \mathbb{Q}^{\alpha, \lambda}\left(\eta, g^{\lambda}\right)}{d \mathbb{Q}^{\alpha}(\eta)}\right)\right]+\mathbb{E}^{\mathbb{Q}^{\alpha, \lambda, \kappa}(\eta, g)}\left[\log \left(\frac{d \mathbb{Q}^{\alpha, \lambda, \kappa}(\eta, g)}{d \mathbb{Q}^{\alpha, \lambda}\left(\eta, g^{\lambda}\right)}\right)\right]\right) \\
= & \frac{1}{\varphi_{\lambda}} \mathbb{E}^{\mathbb{Q}^{\alpha, \lambda, \kappa}(\eta, g)}\left[\log \left(\frac{d \mathbb{Q}^{\alpha, \lambda, \kappa}(\eta, g)}{d \mathbb{Q}^{\alpha}(\eta)}\right)\right] \\
\geq & 0
\end{aligned}
$$

Combining these two inequalities gives the desired inequality:

$$
\mathbb{E}^{\mathbb{Q}^{\alpha, \lambda, \kappa}(\eta, g)}\left[\mathcal{H}\left(\mathbb{Q}^{\alpha, \lambda, \kappa}(\eta, g) \mid \mathbb{P}\right)\right] \geq 0
$$

The case of $\varphi_{\kappa}>\varphi_{\lambda}$ is handled similarly.

In the following sections, the functional $\mathcal{K}_{ \pm}^{c, d}(a, b)$ defined for functions $\{a(y), b(y)\}$ and 
constants $\{c, d\}$ :

$$
\begin{aligned}
\mathcal{K}_{ \pm}^{c, d}(a, b)= & \frac{1}{c} \int_{0}^{\infty}\left[-\left(e^{a(y)}-1\right)+a(y) e^{a(y)+b(y)}\right] \lambda^{ \pm} F^{ \pm}(y) d y \\
& +\frac{1}{d} \int_{0}^{\infty}\left[-\left(e^{b(y)}-1\right) e^{a(y)}+b(y) e^{a(y)+b(y)}\right] \lambda^{ \pm} F^{ \pm}(y) d y
\end{aligned}
$$

will appear several times. With this notation, the $\mathbb{Q}^{\alpha, \lambda \kappa}(\eta, g)$-expectation of $\mathcal{H}^{\lambda, \kappa}\left(\mathbb{Q}^{\alpha, \lambda, \kappa}(\eta, g) \mid \mathbb{Q}^{\alpha}(\eta)\right)$ can be written as:

$$
\begin{aligned}
\mathbb{E}^{\mathbb{Q}^{\alpha, \lambda, \kappa}(\eta, g)}\left[\mathcal{H}^{\varphi_{\lambda}, \varphi_{\kappa}}\right. & \left.\left(\mathbb{Q}^{\alpha, \lambda, \kappa}(\eta, g) \mid \mathbb{Q}^{\alpha}(\eta)\right)\right] \\
= & \sum_{i= \pm}\left\{\mathbb{E}^{\mathbb{Q}^{\alpha, \lambda, \kappa}(\eta, g)}\left[\int_{0}^{T} \mathcal{K}_{i}^{\varphi_{\lambda}, \varphi_{\kappa}}\left(g_{u}^{i \lambda}, g_{u}^{i \kappa}\right) d u\right] \mathbb{1}_{\varphi_{\lambda} \geq \varphi_{\kappa}}\right. \\
& \left.+\mathbb{E}^{\mathbb{Q}^{\alpha, \lambda, \kappa}(\eta, g)}\left[\int_{0}^{T} \mathcal{K}_{i}^{\varphi_{\kappa}, \varphi_{\lambda}}\left(g_{u}^{i \kappa}, g_{u}^{i \lambda}\right) d u\right] \mathbb{1}_{\varphi_{\lambda}<\varphi_{\kappa}}\right\},
\end{aligned}
$$

Denote the triple of ambiguity levels as $\psi=\left(\varphi_{\alpha}, \varphi_{\lambda}, \varphi_{\kappa}\right)$. With the decomposition of the total measure change described in Proposition 5 and choice of penalty function given by equation (2.21), the market maker's value function can now be stated:

$$
H^{\alpha, \lambda, \kappa}(t, x, q, S ; \psi)=\sup _{\left(\delta_{u}^{ \pm}\right)_{t \leq u \leq T} \in \mathcal{A}} \inf _{\mathbb{Q} \in \mathcal{Q}^{\alpha, \lambda, \kappa}} \mathbb{E}_{t, x, q, S}^{\mathbb{Q}}\left[X_{T}+q_{T}\left(S_{T}-\ell\left(q_{T}\right)\right)+\mathcal{H}_{t, T}[\mathbb{Q} \mid \mathbb{P}]\right]
$$

Continuing in the usual fashion, the MM's value function satisfies an HJB equation in which the usual ansatz is made, $H^{\alpha, \lambda, \kappa}(t, x, q, S ; \psi)=x+q S+h_{q}^{\psi}(t)$. The resulting equation for $h_{q}^{\psi}(t)$ is

$$
\begin{aligned}
& \partial_{t} h_{q}^{\psi}+\inf _{\eta}\left\{\eta q+\frac{1}{2 \varphi_{\alpha}}\left(\frac{\alpha-\eta}{\sigma}\right)^{2}\right\} \\
&+\sup _{\delta^{+} \geq 0} \inf _{g^{+\lambda}} \inf _{g^{+\kappa} \in \mathcal{G}^{+}}\left\{\lambda^{+}\left[\int_{\delta^{+}}^{\infty} e^{g_{t}^{+\lambda}+g_{t}^{+\kappa}(y)} F^{+}(y) d y\right]\left(\delta^{+}+h_{q-1}^{\psi}-h_{q}^{\psi}\right) \mathbb{1}_{q \neq \underline{q}}\right. \\
&\left.+\mathcal{K}_{+}^{\varphi_{\lambda}, \varphi_{\kappa}}\left(g_{t}^{+\lambda}, g_{t}^{+\kappa}\right) \mathbb{1}_{\varphi_{\lambda} \geq \varphi_{\kappa}}+\mathcal{K}_{+}^{\varphi_{\kappa}, \varphi_{\lambda}}\left(g_{t}^{+\kappa}, g_{t}^{+\lambda}\right) \mathbb{1}_{\varphi_{\lambda}<\varphi_{\kappa}}\right\} \\
&+\sup _{\delta^{-} \geq 0} \inf _{g^{-\lambda}} \inf _{g^{-\kappa} \in \mathcal{G}^{-}}\left\{\lambda^{-}\left[\int_{\delta^{-}}^{\infty} e^{g_{t}^{-\lambda}+g_{t}^{-\kappa}(y)} F^{-}(y) d y\right]\left(\delta^{-}+h_{q+1}^{\psi}-h_{q}^{\psi}\right) \mathbb{1}_{q \neq \bar{q}}\right. \\
&\left.\quad+\mathcal{K}_{-}^{\varphi_{\lambda}, \varphi_{\kappa}}\left(g_{t}^{-\lambda}, g_{t}^{-\kappa}\right) \mathbb{1}_{\varphi_{\lambda} \geq \varphi_{\kappa}}+\mathcal{K}_{-}^{\varphi_{\kappa}, \varphi_{\lambda}}\left(g_{t}^{-\kappa}, g_{t}^{-\lambda}\right) \mathbb{1}_{\varphi_{\lambda}<\varphi_{\kappa}}\right\}=0 .
\end{aligned}
$$


where the sets $\mathcal{G}^{ \pm}$are:

$$
\mathcal{G}^{ \pm}=\left\{g: \int_{0}^{\infty} e^{g(y)} F^{ \pm}(y) d y=1\right\}
$$

Proposition 7 (Optimal Feedback Controls). The optimal feedback controls of equation (2.23) are given by

$$
\begin{aligned}
\delta^{+*}(t, q ; \psi) & =\left(\frac{1}{\varphi_{\kappa}} \log \left(1+\frac{\varphi_{\kappa}}{\kappa^{+}}\right)-h_{q-1}^{\psi}(t)+h_{q}^{\psi}(t)\right)_{+}, q \neq \underline{q}, \\
\delta^{-*}(t, q ; \psi) & =\left(\frac{1}{\varphi_{\kappa}} \log \left(1+\frac{\varphi_{\kappa}}{\kappa^{-}}\right)-h_{q+1}^{\psi}(t)+h_{q}^{\psi}(t)\right)_{+}, q \neq \bar{q}, \\
g^{\lambda \pm *}(t, q ; \psi) & =\frac{\varphi_{\lambda}}{\varphi_{\kappa}} \log \left(1-e^{-\kappa^{ \pm} \delta^{ \pm *}(t, q ; \psi)}\left(1-e^{-\varphi_{\kappa}\left(\delta^{ \pm *}(t, q ; \psi)+h_{q \mp 1}^{\psi}(t)-h_{q}^{\psi}(t)\right)}\right)\right), \\
g^{\kappa \pm *}(t, q, y ; \psi) & =-\log \left(1-e^{-\kappa^{ \pm} \delta^{ \pm *}(t, q ; \psi)}\left(1-e^{-\varphi_{\kappa}\left(\delta^{ \pm *}(t, q ; \psi)+h_{q \mp 1}^{\psi}(t)-h_{q}^{\psi}(t)\right)}\right)\right) \\
\eta^{*}(t, q ; \psi) & \quad-\varphi_{\kappa}\left(\delta^{ \pm *}(t, q ; \psi)+h_{q \mp 1}^{\psi}(t)-h_{q}^{\psi}(t)\right) \mathbb{1}_{y \geq \delta} \delta^{ \pm *}(t, q ; \psi)
\end{aligned}
$$

Proof. See Appendix B.1.

\section{Discussion and Financial Interpretation}

Substituting the optimal value of $\eta^{*}(t, q ; \psi), g^{\lambda \pm *}(t, q ; \psi)$, and $g^{\kappa \pm *}(t, q, y ; \psi)$ into equation (2.23) results in a system of ODE's for $h_{q}^{\psi}(t)$ that is solved numericaly:

$$
\begin{aligned}
& \partial_{t} h_{q}^{\psi}+\alpha q-\frac{1}{2} \varphi_{\alpha} \sigma^{2} q^{2} \\
&+\sup _{\delta^{+} \geq 0}\left\{\frac{\lambda^{+}}{\varphi_{\lambda}}\left(1-\exp \left\{\frac{\varphi_{\lambda}}{\varphi_{\kappa}} \log \left(1-e^{-\kappa^{+} \delta^{+}}+e^{-\kappa^{+} \delta^{+}-\varphi_{\kappa}\left(\delta^{+}+h_{q-1}^{\psi}-h_{q}^{\psi}\right)}\right)\right\}\right)\right\} \mathbb{1}_{q \neq \underline{q}} \\
&+\sup _{\delta^{-} \geq 0}\left\{\frac{\lambda^{-}}{\varphi_{\lambda}}\left(1-\exp \left\{\frac{\varphi_{\lambda}}{\varphi_{\kappa}} \log \left(1-e^{-\kappa^{-} \delta^{-}}+e^{-\kappa^{-} \delta^{-}-\varphi_{\kappa}\left(\delta^{-}+h_{q+1}^{\psi}-h_{q}^{\psi}\right)}\right)\right\}\right)\right\} \mathbb{1}_{q \neq \bar{q}}=0, \\
& h_{q}^{\psi}(T)=-q \ell(q) .
\end{aligned}
$$

There is no known analytical solution to this equation, but a classical solution exists (see Proposition 10). Solving numerically allows Figure 2.7 to show the optimal sell spreads for different levels of mixed ambiguity. Note that spreads always decrease at maturity due 
to the non-zero level of ambiguity on fill probability. However, the optimal behaviour of the spreads at early times does not exhibit a uniform increasing or decreasing behaviour. The type of modification that the MM makes to their spread relative to the ambiguity neutral case depends on the levels of ambiguity to all three possible factors. Also, as would be intuitively expected, increasing the value of an ambiguity level strengthens the behaviour as seen in previous sections associated with that parameter. For example, in Figure 2.7 (a), the large value of $\varphi_{\lambda}$ relative to $\varphi_{\kappa}$ alters the spreads in such a way that at early times the ambiguity with respect to $\mathrm{MO}$ arrival rate is more apparent than ambiguity with respect to fill probability (if fill probability had a stronger effect, then all of the spreads would decrease as in Section 2.2.2). In Figure 2.7 (b), the larger value of $\varphi_{\kappa}$ amplifies the behaviour associated with that type of ambiguity to a point where that aspect of the behaviour (a downward shift in spread) outweighs the effects of the other types of ambiguity.

If the optimal spreads $\delta^{+*}(t, q ; \psi)$ remain positive, then substituting them into the expressions for $g^{\lambda \pm *}(t, q ; \psi)$ and $g^{\kappa \pm *}(t, q, y ; \psi)$, as well as equation $(2.24)$ give them simplified forms:

$$
\begin{aligned}
g^{\lambda \pm *}(t, q ; \psi) & =\frac{\varphi_{\lambda}}{\varphi_{\kappa}} \log \left(1-\left(1+\frac{\kappa^{ \pm}}{\varphi_{\kappa}}\right)^{-1} e^{-\kappa^{ \pm} \delta^{ \pm *}(t, q ; \psi)}\right) \\
g^{\kappa \pm *}(t, q, y ; \psi) & =-\log \left(1-\left(1+\frac{\kappa^{ \pm}}{\varphi_{\kappa}}\right)^{-1} e^{-\kappa^{ \pm} \delta^{ \pm *}(t, q ; \psi)}\right)-\log \left(1+\frac{\varphi_{\kappa}}{\kappa^{ \pm}}\right) \mathbb{1}_{y>\delta^{ \pm *}(t, q ; \psi)}
\end{aligned}
$$

and

$$
\begin{aligned}
\partial_{t} h_{q}^{\psi}+\alpha q & -\frac{1}{2} \varphi_{\alpha} \sigma^{2} q^{2} \\
+ & \frac{\lambda^{+}}{\varphi_{\lambda}}\left\{1-\exp \left\{\frac{\varphi_{\lambda}}{\varphi_{\kappa}} \log \left(1-\left(1+\frac{\kappa^{+}}{\varphi_{\kappa}}\right)^{-1}\left(1+\frac{\varphi_{\kappa}}{\kappa^{+}}\right)^{-\frac{\kappa^{+}}{\varphi_{\kappa}}} e^{\kappa^{+}\left(h_{q-1}^{\psi}-h_{q}^{\psi}\right)}\right)\right\}\right\} \mathbb{1}_{q \neq \underline{q}} \\
+ & \frac{\lambda^{-}}{\varphi_{\lambda}}\left\{1-\exp \left\{\frac{\varphi_{\lambda}}{\varphi_{\kappa}} \log \left(1-\left(1+\frac{\kappa^{-}}{\varphi_{\kappa}}\right)^{-1}\left(1+\frac{\varphi_{\kappa}}{\kappa^{-}}\right)^{-\frac{\kappa^{-}}{\varphi_{\kappa}}} e^{\kappa^{-}\left(h_{q+1}^{\psi}-h_{q}^{\psi}\right)}\right)\right\}\right\} \mathbb{1}_{q \neq \bar{q}}=0 .
\end{aligned}
$$



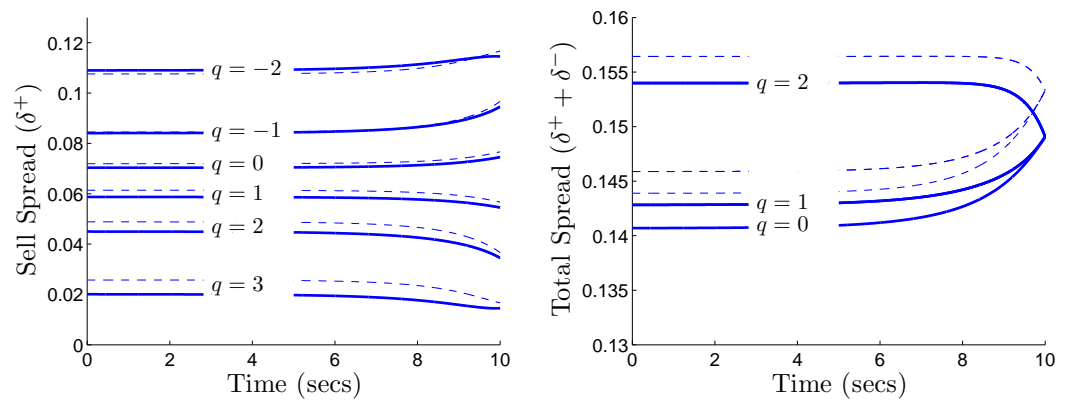

(a) ambiguity levels: $\varphi_{\alpha}=0, \varphi_{\lambda}=6, \varphi_{\kappa}=1$
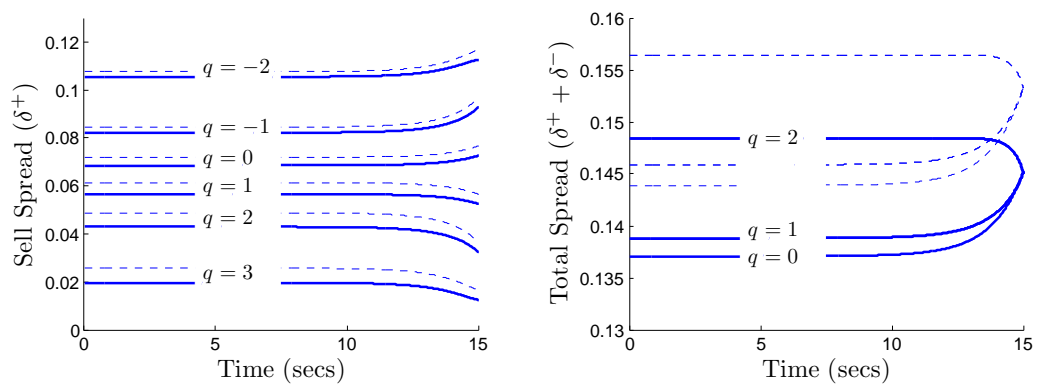

(b) ambiguity levels: $\varphi_{\alpha}=10, \varphi_{\lambda}=2, \varphi_{\kappa}=2$

Figure 2.7: Optimal sell and total spreads for an MM who utilizes mixed ambiguity (dashed lines are ambiguity neutral spreads). Parameter values are $\kappa^{ \pm}=15, \lambda^{ \pm}=2$, $\sigma=0.01, \alpha=0, \ell(q)=\theta q, \theta=0.01, \bar{q}=-\underline{q}=3$ and $T=10$. 


\subsection{Closed Form Solutions}

Suppose the MM has equal levels of ambiguity on the arrival rate of MO's and the fill probability of their posted limit orders. This corresponds to the case $\varphi_{\lambda}=\varphi_{\kappa}=\varphi$. As previously noted, both versions of the penalty function defined in equation (2.22) agree in this case, and the full penalty is equal to:

$$
\mathcal{H}\left(\mathbb{Q}^{\alpha, \lambda, \kappa}(\eta, g) \mid \mathbb{P}\right)=\frac{1}{\varphi_{\alpha}} \log \left(\frac{d \mathbb{Q}^{\alpha}(\eta)}{d \mathbb{P}}\right)+\frac{1}{\varphi} \log \left(\frac{d \mathbb{Q}^{\alpha, \lambda, \kappa}(\eta, g)}{d \mathbb{Q}^{\alpha}(\eta)}\right)
$$

At this point, one can either proceed as usual and derive the HJB equation satisfied by the value function, or consider the case $\varphi_{\lambda}=\varphi_{\kappa}=\varphi$ in equation (2.24). Both procedures naturally yield the same system of equations to be solved:

$$
\begin{aligned}
\partial_{t} h_{q}^{\psi}+\alpha q-\frac{1}{2} \varphi_{\alpha} \sigma^{2} q^{2} & +\sup _{\delta^{+} \geq 0}\left\{\frac{\lambda^{+}}{\varphi} e^{-\kappa^{+} \delta^{+}}\left(1-e^{-\varphi\left(\delta^{+}+h_{q-1}^{\psi}-h_{q}^{\psi}\right)}\right)\right\} \mathbb{1}_{q \neq \underline{q}} \\
& +\sup _{\delta^{-} \geq 0}\left\{\frac{\lambda^{-}}{\varphi} e^{-\kappa^{-} \delta^{-}}\left(1-e^{-\varphi\left(\delta^{-}+h_{q+1}^{\psi}-h_{q}^{\psi}\right)}\right)\right\} \mathbb{1}_{q \neq \bar{q}}=0
\end{aligned}
$$

The optimal spreads have the same feedback form as in Proposition 7. If the optimal spreads remain positive for all $t$ and $q$, then substituting them into equation (2.26) yields:

$$
\partial_{t} h_{q}^{\psi}+\alpha q-\frac{1}{2} \varphi_{\alpha} \sigma^{2} q^{2}+\frac{\xi^{+}}{\kappa^{+}} e^{-\kappa^{+}\left(-h_{q-1}^{\psi}+h_{q}^{\psi}\right)} \mathbb{1}_{q \neq \underline{q}}+\frac{\xi^{-}}{\kappa^{-}} e^{-\kappa^{-}\left(-h_{q+1}^{\psi}+h_{q}^{\psi}\right)} \mathbb{1}_{q \neq \bar{q}}=0,
$$

where

$$
\xi^{ \pm}=\left(1+\frac{\varphi}{\kappa^{ \pm}}\right)^{-\left(1+\frac{\kappa^{ \pm}}{\varphi}\right)} \lambda^{ \pm}
$$

Proposition 8 (Solving for $h_{q}^{\psi}(t)$ in equation $(2.27)$ ). If $\kappa^{ \pm}=\kappa$, write $\omega_{q}^{\psi}=e^{\kappa h_{q}^{\psi}(t)}$. Define the vector

$\boldsymbol{\omega}_{q}^{\psi}(t)=\left[\omega_{\bar{q}}^{\psi}(t), \omega_{\bar{q}-1}^{\psi}(t), \ldots, \omega_{\underline{q}}^{\psi}(t)\right]^{\prime}$. Also, let $\mathbf{A}^{\psi}$ denote the $(\bar{q}-\underline{q}+1)$-square matrix with rows labelled from $\bar{q}$ to $\underline{q}$ with entries

$$
\mathbf{A}_{i, q}^{\psi}=\left\{\begin{aligned}
\alpha \kappa q-\frac{1}{2} \varphi_{\alpha} \sigma^{2} q^{2}, & i=q \\
\xi^{+}, & i=q-1 \\
\xi^{-}, & i=q+1 \\
0, & \text { otherwise }
\end{aligned}\right.
$$


Then,

$$
\boldsymbol{\omega}^{\psi}(t)=e^{\mathbf{A}^{\psi}(T-t)} \boldsymbol{\omega}^{\psi}(T)
$$

where $\omega_{q}^{\psi}(T)=e^{-\kappa q \ell(q)}$.

Proof. See Appendix B.1.

Note that the closed form expression in Proposition 8 also applies when $\varphi_{\alpha}=0$ or $\varphi=0$. In the latter case, the limiting value of $\xi^{ \pm}=e^{-1} \lambda^{ \pm}$is used in the matrix $\mathbf{A}^{\psi}$. This result ensures a closed form analytical solution in any case of the robust market making problem when $\kappa^{+}=\kappa^{-}, \varphi_{\lambda}=\varphi_{\kappa}$, and the corresponding optimal spreads remain positive. Further, note that the form of equation (2.27) is the same as equation (2.15) except with $\lambda^{ \pm}$replaced with $e \xi^{ \pm}$. It may be tempting to conclude that the problem in this case is equivalent to that of an agent that has no ambiguity on market order arrivals or maximum price execution, but with a modified reference arrival rate. This is only half true, as the values of the function $h_{q}^{\psi}(t)$ will be the same, and hence also the full value function $H(t, x, q, S ; \psi)$, but the optimal spreads $\delta^{ \pm *}(t, q ; \psi)$ will have different values (compare the feedback forms to see the difference).

Proposition 9 (Symmetry in spreads). If $\alpha=0, \lambda^{+}=\lambda^{-}, \kappa^{+}=\kappa^{-}, \bar{q}=-\underline{q}$, and $q \ell(q)$ is an even function, then $\delta^{+*}(t, q ; \psi)=\delta^{-*}(t,-q ; \psi)$.

Proof. Under the stated conditions, equation (2.24) and the associated terminal conditions are invariant under $q \mapsto-q, \pm \mapsto \mp$, and $h_{q}^{\psi}(t) \mapsto h_{-q}^{\psi}(t)$. This means $h_{q}^{\psi}(t)=h_{-q}^{\psi}(t)$, and so from the feedback form of $\delta^{ \pm *}(t, q ; \psi)$, the result follows.

All of the figures in this chapter that show the optimal spreads for various levels of ambiguity only show the sell spreads, $\delta^{+*}$. It was claimed that showing the buy spreads, $\delta^{-*}$, would be redundant. This is now justified by Proposition 9 as all of the parameter selections satisfied the symmetry conditions given in the proposition. 


\subsection{Verification Theorem}

Most of the equations to this point have required numerical solutions in order to study the behaviour of the optimal spreads under ambiguity. Although this analysis has depended heavily upon numerics, this section will show two important results. First, all of the differential equations for functions of the form $h_{q}^{\psi}(t)$ have classical solutions, and therefore so do the value functions $H^{\psi}(t, x, q, S)$. This also implies that the optimal spreads, $\delta^{ \pm *}(t, q ; \psi)$ are classical functions. Second, a verification theorem will show that the classical solutions for $H^{\psi}(t, x, q, S)$ are indeed solutions to the original optimization problem in each case.

Proposition 10 (Existence and Uniqueness of Solutions). Equations (2.4), (2.9), (2.12), (2.14), and (2.24) have unique classical solutions for all $t \in[0, T]$ and $q \in$ $\{\underline{q}, \ldots, \bar{q}\}$.

Proof. See Section B.1.1 in Appendix B.1.

Theorem 11 (Verification Theorem). Let $h_{q}^{\psi}(t)$ be a classical solution to

$$
\begin{aligned}
& \partial_{t} h_{q}^{\psi}+\inf _{\eta}\left\{\eta q+\frac{1}{2 \varphi_{\alpha}}\left(\frac{\alpha-\eta}{\sigma}\right)^{2}\right\} \\
&+\sup _{\delta^{+} \geq 0} \inf _{g^{+\lambda}} \inf _{g^{+\kappa} \in \mathcal{G}^{+}}\left\{\lambda^{+}\left[\int_{\delta^{+}}^{\infty} e^{g_{t}^{+\lambda}+g_{t}^{+\kappa}(y)} F^{+}(y) d y\right]\left(\delta^{+}+h_{q-1}^{\psi}-h_{q}^{\psi}\right) \mathbb{1}_{q \neq \underline{q}}\right. \\
&\left.\quad+\mathcal{K}_{+}^{\varphi_{\lambda}, \varphi_{\kappa}}\left(g_{t}^{+\lambda}, g_{t}^{+\kappa}\right) \mathbb{1}_{\varphi_{\lambda} \geq \varphi_{\kappa}}+\mathcal{K}_{+}^{\varphi_{\kappa}, \varphi_{\lambda}}\left(g_{t}^{+\kappa}, g_{t}^{+\lambda}\right) \mathbb{1}_{\varphi_{\lambda}<\varphi_{\kappa}}\right\} \\
&+\sup _{\delta^{-} \geq 0} \inf _{g^{-\lambda}} \inf _{g^{-\kappa} \in \mathcal{G}^{-}}\left\{\lambda^{-}\left[\int_{\delta^{-}}^{\infty} e^{g_{t}^{-\lambda}+g_{t}^{-\kappa}(y)} F^{-}(y) d y\right]\left(\delta^{-}+h_{q+1}^{\psi}-h_{q}^{\psi}\right) \mathbb{1}_{q \neq \bar{q}}\right. \\
&\left.\quad+\mathcal{K}_{-}^{\varphi_{\lambda}, \varphi_{\kappa}}\left(g_{t}^{-\lambda}, g_{t}^{-\kappa}\right) \mathbb{1}_{\varphi_{\lambda} \geq \varphi_{\kappa}}+\mathcal{K}_{-}^{\varphi_{\kappa}, \varphi_{\lambda}}\left(g_{t}^{-\kappa}, g_{t}^{-\lambda}\right) \mathbb{1}_{\varphi_{\lambda}<\varphi_{\kappa}}\right\}=0,
\end{aligned}
$$

subject to $h_{q}^{\psi}(T)=-q \ell(q)$. Then $H^{\alpha, \lambda, \kappa}(t, x, q, S ; \psi)=x+q S+h_{q}^{\psi}(t)$ is the value function to the agent's control problem:

$$
H^{\alpha, \lambda, \kappa}(t, x, q, S ; \psi)=\sup _{\left(\delta_{s}^{ \pm}\right)_{t \leq s \leq T} \in \mathcal{A}} \inf _{\mathbb{Q} \in \mathcal{Q}^{\alpha, \lambda, \kappa}} \mathbb{E}_{t, x, q, S}^{\mathbb{Q}}\left[X_{T}+q_{T}\left(S_{T}-\ell\left(q_{T}\right)\right)+\mathcal{H}_{t, T}[\mathbb{Q} \mid \mathbb{P}]\right]
$$

with $\mathcal{H}_{t, T}[\mathbb{Q} \mid \mathbb{P}]$ defined as in equation (2.21). 
Proof. See Section B.1.1 in Appendix B.1.

\subsection{Numerical Implementation}

With analytic solutions available in only a special case of the parameter set, numerical solutions must be relied upon in order to investigate the effect of ambiguity on optimal spreads. An outline of the numerical algorithm used to solve the associated system of ODE's is presented here. The equations which are of concern in this section are (2.4), (2.9), (2.12), (2.14), and (2.24). With the exception of equation (2.4) which is the classical case of no ambiguity, each of these equations arises from substituting the optimal $g^{ \pm \lambda *}$, $g^{ \pm \kappa *}(y)$, and $\eta^{*}$ where applicable. The resulting equations can all be written in the form:

$$
\begin{aligned}
\partial_{t} h_{q}+\alpha q-\frac{1}{2} \varphi_{\alpha} \sigma^{2} q^{2}+\sup _{\delta^{+} \geq 0} f\left(h_{q-1}, h_{q}, \delta^{+}\right) \mathbb{1}_{q \neq \underline{q}}+\sup _{\delta^{-} \geq 0} f\left(h_{q+1}, h_{q}, \delta^{-}\right) \mathbb{1}_{q \neq \bar{q}} & =0 \\
h_{q}(T) & =-q \ell(q)
\end{aligned}
$$

where the function $f$ is chosen to appropriately reflect which type of penalty function is applied. This equation is treated in a fully explicit method. The time interval $[0, T]$ is subdivided into $N$ subintervals of equal length, and set $\Delta t=\frac{T}{N}$ and $t_{n}=n \Delta t$. Also set $h_{q, n}=h_{q}\left(t_{n}\right)$. Then $h_{,, n-1}$ is computed from $h_{,, n}$ via the process outlined in the following pseudocode:

$$
\begin{aligned}
& \operatorname{if}\left(\varphi_{\kappa}>0\right) \\
& \quad \delta^{+}=\max \left(1 / \varphi_{\kappa} \log \left(1+\varphi_{\kappa} / \kappa^{+}\right)-\mathrm{h}[\bar{q}-1, \mathrm{n}]+\mathrm{h}[\bar{q}, \mathrm{n}], 0\right) \\
& \text { else } \\
& \quad \delta^{+}=\max \left(1 / \kappa^{+}-\mathrm{h}[\bar{q}-1, \mathrm{n}]+\mathrm{h}[\bar{q}, \mathrm{n}], 0\right) \\
& \mathrm{h}[\bar{q}, \mathrm{n}-1]=\mathrm{h}[\bar{q}, \mathrm{n}]+\Delta \mathrm{t} *\left(\mathrm{f}\left(\mathrm{h}[\bar{q}-1, \mathrm{n}], \mathrm{h}[\bar{q}, \mathrm{n}], \delta^{+}\right)+\alpha \bar{q}-\frac{1}{2} \varphi_{\alpha} \sigma^{2} \bar{q}^{2}\right) \\
& \operatorname{if}\left(\varphi_{\kappa}>0\right) \\
& \quad \delta^{-}=\max \left(1 / \varphi_{\kappa} \log \left(1+\varphi_{\kappa} / \kappa^{-}\right)-\mathrm{h}[\underline{q}+1, \mathrm{n}]+\mathrm{h}[\underline{q}, \mathrm{n}], 0\right) \\
& \text { else } \\
& \quad \delta^{-}=\max \left(1 / \kappa^{-}-\mathrm{h}[\underline{q}+1, \mathrm{n}]+\mathrm{h}[\underline{q}, \mathrm{n}], 0\right) \\
& \mathrm{h}[\underline{q}, \mathrm{n}-1]=\mathrm{h}[\underline{q}, \mathrm{n}]+\Delta \mathrm{t} *\left(\mathrm{f}\left(\mathrm{h}[\underline{q}+1, \mathrm{n}], \mathrm{h}[\underline{q}, \mathrm{n}], \delta^{-}\right)+\alpha \underline{q}-\frac{1}{2} \varphi_{\alpha} \sigma^{2} \underline{q}^{2}\right)
\end{aligned}
$$




$$
\begin{aligned}
& \text { for }(j=\underline{q}+1 \text { to } \bar{q}-1) \\
& \text { if }\left(\varphi_{\kappa}>0\right) \\
& \delta^{+}=\max \left(1 / \varphi_{\kappa} \log \left(1+\varphi_{\kappa} / \kappa^{+}\right)-\mathrm{h}[j-1, \mathrm{n}]+\mathrm{h}[\mathrm{j}, \mathrm{n}], 0\right) \\
& \delta^{-}=\max \left(1 / \varphi_{\kappa} \log \left(1+\varphi_{\kappa} / \kappa^{-}\right)-\mathrm{h}[j+1, \mathrm{n}]+\mathrm{h}[\mathrm{j}, \mathrm{n}], 0\right) \\
& \text { else } \\
& \delta^{+}=\max \left(1 / \kappa^{+}-\mathrm{h}[j-1, \mathrm{n}]+\mathrm{h}[j, \mathrm{n}], 0\right) \\
& \delta^{-}=\max \left(1 / \kappa^{-}-h[j+1, n]+h[j, n], 0\right) \\
& h[j, n-1]=h[j, n]+\Delta t *\left(f\left(h[j-1, n], h[j, n], \delta^{+}\right)\right. \\
& \left.+f\left(h[j+1, n], h[j, n], \delta^{-}\right)+\alpha j-\frac{1}{2} \varphi_{\alpha} \sigma^{2} j^{2}\right)
\end{aligned}
$$

This is a fully explicit method that solves for $h_{q, n}$. Although more sophisticated numerical techniques can be used, convergence of solutions using this method (with $\Delta t=\frac{1}{3000}$ ) is observed to be fast enough to easily observe the qualitative behaviour of the solutions.

\subsection{Simulation results}

This section will show the effect of different levels of ambiguity on the performance of an agent posting according to the corresponding optimal strategy. The important feature of this section is the relationship between the data generating process and the calibrated reference model. Ambiguity aversion as outlined in this work is a method of protecting an agent from model misspecification, and so it doesn't make sense for the data generating process to be based on the reference model. It is reasonable to think that the parameters of the reference model $\left(\alpha, \lambda^{ \pm}\right.$, and $\left.\kappa^{ \pm}\right)$can be estimated from historical data and be set equal to their long term averages. This is how the reference model will be related to the data generating process in these simulations.

Midprice: The midprice will evolve according to:

$$
\begin{aligned}
d S_{t} & =\alpha_{t} d t+\sigma d W_{t}, \\
d \alpha_{t} & =-\beta_{\alpha} d t+\epsilon^{+} d M_{t}^{+}-\epsilon^{-} d M_{t}^{-},
\end{aligned}
$$


where $\epsilon^{+}=\epsilon^{-}$are positive constants. This relationship between market orders and the drift will generally cause the midprice to move upwards following a market buy order and downwards after a market sell order. This is typical and temporary behaviour of the midprice following the execution of a market order.

Market order arrivals: The arrival of market orders follows a bivariate Hawkes process with intensities satisfying:

$$
d \lambda_{t}^{ \pm}=\beta_{\lambda}\left(\theta_{\lambda}-\lambda_{t}^{ \pm}\right) d t+\eta_{\lambda} d M_{t}^{ \pm}+\nu_{\lambda} d M_{t}^{\mp}
$$

These dynamics will induce self-exciting behaviour of market orders, which results in trade clustering. This is a feature that can not arise with the Poisson dynamics of the reference model.

Fill probability: Finally, the fill probability of a posted limit order will still be exponential, but with stochastically changing parameters satisfying:

$$
d \kappa_{t}^{ \pm}=\beta_{\kappa}\left(\theta_{\kappa}-\kappa_{t}^{ \pm}\right) d t+\eta_{\kappa} d M_{t}^{ \pm}+\nu_{\kappa} d M_{t}^{\mp}
$$

This reflects the stochastic nature of the depth of the limit order book, which was a static feature of the reference model.

Numerical values used for these simulations are given by:

\begin{tabular}{|r|r|r|r|r|r|r|r|r|r|r|}
\hline$\sigma$ & $\epsilon^{ \pm}$ & $\beta_{\alpha}$ & $\theta_{\lambda}$ & $\beta_{\lambda}$ & $\eta_{\lambda}$ & $\nu_{\lambda}$ & $\theta_{\kappa}$ & $\beta_{\kappa}$ & $\eta_{\kappa}$ & $\nu_{\kappa}$ \\
\hline 0.01 & 0.001 & 1 & 0.2 & $70 / 9$ & 5 & 2 & 15 & $7 / 6$ & 5 & 2 \\
\hline
\end{tabular}

Table 2.1: Parameter values used to generate midprice, LOB, and MOs dynamics

The reference model is then calibrated as follows:

$$
\alpha=\lim _{t \rightarrow \infty} \mathbb{E}\left[\alpha_{t} \mid \mathcal{F}_{0}\right], \quad \lambda^{ \pm}=\lim _{t \rightarrow \infty} \mathbb{E}\left[\lambda_{t}^{ \pm} \mid \mathcal{F}_{0}\right], \quad \kappa^{ \pm}=\lim _{t \rightarrow \infty} \mathbb{E}\left[\kappa_{t}^{ \pm} \mid \mathcal{F}_{0}\right]
$$

which give numerical values $\alpha=0, \lambda^{ \pm}=2, \kappa^{ \pm}=27$. The computation of these values is done using the method outlined in Cartea et al. (2014). Using these parameters in the reference model, the market maker's spreads can be computed for various levels of ambiguity. Once spreads are computed, the data generating process is used to compute 


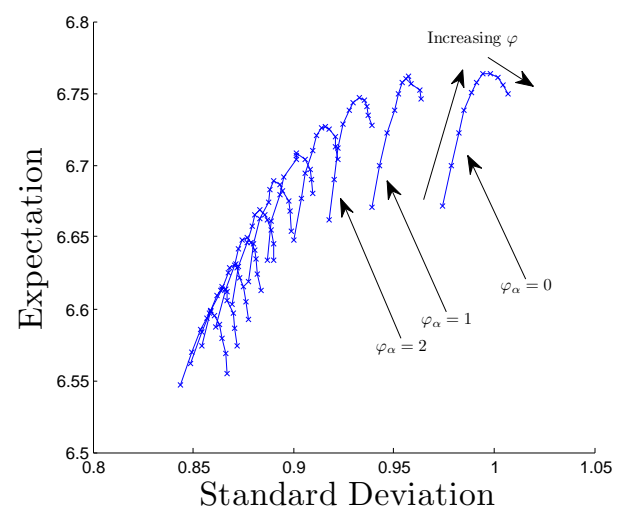

Figure 2.8: Expectation and standard deviation for various levels of ambiguity parameters when trading strategies are executed for 300 seconds on the simulated process outlined above. The $\varphi_{\alpha}$ parameter ranges from 0 to 10 and $\varphi_{\lambda}=\varphi_{\kappa}=\varphi$ ranges from 0 to 20 .

the market maker's terminal wealth and inventory holdings. Any terminal inventory is liquidated at a penalized value of $S_{T}-\theta q_{T}$ per share, where $\theta=0.001$. This simulation is performed 10,000 times to arrive at mean and standard deviations of the market maker's wealth and inventory holdings shown in Figure 2.8.

Each point in Figure 2.8 is intended to represent a Sharpe ratio quantity for that particular combination of ambiguity level parameters. For this particular data generating process, increasing $\varphi_{\alpha}$ appears to have the uniform effect of decreasing both the expected value and standard deviation of terminal wealth. Perhaps this is expected behaviour due to the effect that $\varphi_{\alpha}$ has on the market maker's inventory process: it generally increases the rate of mean reversion to zero inventory. Such behaviour will result in fewer trades being made overall, naturally leading to both smaller mean and standard deviation. As seen in Figure 2.9, not only are there less trades made, but the average spread earned on each trade is affected very little.

The behaviour of the performance as a function of $\varphi$ is less straightforward. Since the $\varphi_{\lambda}$ and $\varphi_{\kappa}$ parameters are being set equal, it is expected that the $\varphi_{\kappa}$ effects will dominate the $\varphi_{\lambda}$ effects (observe Figure 2.7 for similar behaviour). The effect of increasing $\varphi_{\kappa}$ is always to post smaller spreads, which will result in faster overall trading. This is also reflected in Figure 2.9. The effects of $\varphi_{\kappa}$ and $\varphi_{\lambda}$ together on the number of trades and average spread have counteracting effects. One one hand, the larger number of trades would suggest a possible increase in both mean and standard deviation, but the lower average spread per trade has the opposite effect. 


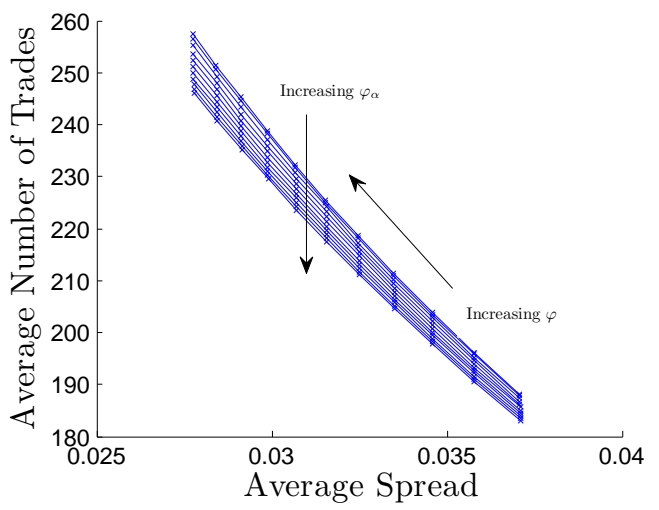

Figure 2.9: Average number of trades and average spread of trades corresponding to parameter ranges of Figure 2.8. 


\section{Chapter 3}

\section{Robust Liquidation}

In the previous chapter, the effects of model uncertainty on the trading strategy of a market maker were examined. While the market maker provides liquidity in return for earning the total spread after their inventory completes a round trip, there are other tasks that agents undertake within the same trading setting for different reasons. An example that is investigated here is that of optimally liquidating a large number of shares in a finite time period. Various approaches to this problem that do not incorporate the effects of model uncertainty have been taken in the past. See Almgren (2003) for an example where the agent's actions use continuously executed market orders that have an impact on the dynamics of the market. See Kharroubi and Pham (2010) in which trading occurs in discrete-time. In Bayraktar and Ludkovski (2011), the problem is considered in the case of illiquid markets.

This optimization problem can arise in a number of different situations. For example, an agent may be required to rebalance a hedging position by selling a certain number of shares of the asset by the end of the trading day. The requirement to liquidate a number of shares is also open for a more natural use of the agent placing market orders in addition to limit orders, and so this setting allows for the investigation of how ambiguity aversion affects the strategy for optimal market order execution. The formulation of the problem in this chapter can equally be applied to an optimal acquisition problem, but due to the similarities to liquidation, only the latter task is investigated here. 


\subsection{Reference Model}

The reference model is structured in a similar manner to the previously considered market making problem. It is modified in such a way that it becomes a one sided model; only the behaviour on one side of the limit order book is of interest.

Midprice: The midprice $S_{t}$ satisfies:

$$
d S_{t}=\alpha d t+\sigma d W_{t}
$$

where $\alpha$ and $\sigma>0$ are constants and $\left(W_{t}\right)_{0 \leq t \leq T}$ is a standard Brownian motion.

Market Orders: As the agent is now undertaking a liquidation problem through the posting of limit orders, only market buy orders are of interest. Thus, only a single Poisson random measure $\mu$ needs to be introduced to the model. The compensator of $\mu$ will be denoted $\nu_{\mathbb{P}}(d y, d t)=\lambda F(y) d y d t$ where $F(y)=\kappa e^{-\kappa y}$. The number of market buy orders that has arrived up to time $t$ is then $M_{t}=\int_{0}^{t} \int_{0}^{\infty} \mu(d y, d s)$.

Limit Order Placement: The agent places limit orders only on the sell side of the limit order book. The distance of the limit order from the midprice is denoted by $\delta_{t}$. In a similar fashion to the market making problem, the agent's limit order is only lifted by a market order that has an execution price greater than $S_{t}+\delta_{t}$. The number of executed limit order is then $N_{t}=\int_{0}^{t} \int_{\delta_{s}}^{\infty} \mu(d y, d s)$.

The appropriate completed filtered probability space to consider is $\left(\Omega, \mathbb{F}, \mathcal{F}=\left\{\mathcal{F}_{t}\right\}_{0 \leq t \leq T}, \mathbb{P}\right)$ where $\mathcal{F}$ is generated by the midprice $\left(S_{t}\right)_{0 \leq t \leq T}$, the number of market orders that have arrived $\left(M_{t}\right)_{0 \leq t \leq T}$, and the number of filled limit orders $\left(N_{t}\right)_{0 \leq t \leq T}$. The agent uses $\delta_{t}$, an $\mathcal{F}_{t}$-predictable process, as their control.

These market dynamics allow the agent's inventory and wealth dynamics to be written. If the agent's inventory is denoted by $q_{t}$, and they begin with a total of $Q$ shares to be liquidated, then $q_{t}=Q-N_{t}$. This implies:

$$
d q_{t}=-d N_{t}
$$

Immediately after a market order lifts the agent's limit order, the agent's wealth increases 
by an amount equal to the trade value $S_{t}+\delta_{t}$. The wealth, $X_{t}$, therefore, has dynamics:

$$
d X_{t}=\left(S_{t}+\delta_{t^{-}}\right) d N_{t}
$$

Unlike the market making problem, the agent does not set minimum and maximum inventory values due to capital constraints. The minimum and maximum values of inventory are dictated by the liquidation problem itself. The agent begins with the maximum value of inventory, $Q$, and trades in a single direction towards the minimum value of inventory, 0 . Once the agent reaches zero inventory, the liquidation is complete and trading ceases. The agent remains risk-neutral so that the effects of model uncertainty can be isolated from those of risk aversion. The agent's optimization problem is to select the strategy $\left(\delta_{t}\right)_{0 \leq t \leq T}$ which maximizes the expected terminal wealth:

$$
H(t, x, q, S)=\sup _{\left(\delta_{s}\right)_{t \leq s \leq T} \in \mathcal{A}} \mathbb{E}_{t, x, q, S}^{\mathbb{P}}\left[X_{\tau \wedge T}+q_{\tau \wedge T}\left(S_{\tau \wedge T}-\ell\left(q_{\tau \wedge T}\right)\right)\right]
$$

where $\tau=\inf \left\{t: q_{t}=0\right\}, T$ is the terminal time of the strategy, $q_{T}$ final inventory, $\mathbb{E}_{t, x, q, S}^{\mathbb{P}}[\cdot]$ denotes $\mathbb{P}$ expectation conditional on $X_{t^{-}}=x, q_{t^{-}}=q$ and $S_{t}=S$, and $\mathcal{A}$ denotes the set of admissible strategies which are non-negative $\mathcal{F}_{t}$-predictable processes. Moreover, the function $\ell\left(q_{T}\right)$, with $\ell(0)=0$ and $\ell(q)$ increasing in $q$, is a liquidation penalty which consists of fees and market impact costs when the agent unwinds terminal inventory. For example $\ell(q)=\theta q$ represents a linear impact when liquidating $q$ shares. Note that since $q_{\tau}=0$, this gives $X_{\tau}+q_{\tau \wedge T}\left(S_{\tau \wedge T}-\ell\left(q_{\tau \wedge T}\right)\right)=X_{\tau}$, and so $H(t, x, 0, S)=$ $x$.

The general form of the HJB equation satisfied by $H(t, x, q, S)$ is the same as that of the market making problem, but the infinitesimal generator will have a different form because the liquidation problem only involves one side of the limit order book.

$$
\partial_{t} H+\sup _{\delta \geq 0} \mathcal{L} H=0
$$

where $\mathcal{L}$ is the infinitesimal generator of the underlying state variables given by:

$$
\mathcal{L} H(t, x, q, S)=\alpha \partial_{S} H+\frac{1}{2} \sigma^{2} \partial_{S S} H+\lambda e^{-\kappa \delta} \mathcal{D} H \mathbb{1}_{q \neq 0}
$$

where the operator $\mathcal{D}$ acts as follows:

$$
\mathcal{D} H=H(t, x+(S+\delta), q-1, S)-H(t, x, q, S),
$$


and subject to the terminal and boundary conditions

$$
\begin{aligned}
H(T, x, q, S) & =x+q(S-\ell(q)) \\
H(t, x, 0, S) & =x
\end{aligned}
$$

\subsubsection{Feedback controls}

Proceeding similarly as to the market making case, the ansatz $H(t, x, q, S)=x+q S+h_{q}(t)$ is still applicable and results in the following system of equations:

$$
\begin{aligned}
\partial_{t} h_{q}+\alpha q+\sup _{\delta \geq 0}\left\{\lambda e^{-\kappa \delta}\left(\delta+h_{q-1}-h_{q}\right)\right\} \mathbb{1}_{q \neq 0} & =0, \\
h_{q}(T) & =-q \ell(q) \\
h_{0}(t) & =0
\end{aligned}
$$

Proposition 12 (Optimal Feedback Controls). The optimal feedback controls of the HJB equation (3.1) are given by

$$
\delta_{q}^{*}(t)=\left(\frac{1}{\kappa}-h_{q-1}(t)+h_{q}(t)\right)_{+}, \quad q \neq 0,
$$

where $(x)_{+}=\max (x, 0)$.

Proof. Apply first order conditions to the supremum term in equation (3.5). The computations to solve for $\delta$ and check that it is indeed a maximizer are equivalent to those in Proposition 1.

As long as the stated feedback controls remain positive, they can be substituted into equation (3.5) to obtain a non-linear system of equations for $h_{q}(t)$ :

$$
\partial_{t} h_{q}+\alpha q+\frac{\lambda}{\kappa} e^{-\kappa\left(\frac{1}{\kappa}-h_{q-1}+h_{q}\right)} \mathbb{1}_{q \neq 0}=0,
$$

along with terminal conditions $h_{q}(T)=-q \ell(q)$ and boundary condition $h_{0}(t)=0$. This equation has a closed form analytical solution which will be discussed in Section (3.3).

In Figure 3.1, the optimal spread to be posted by the agent is plotted for each value of 
inventory. Clearly the qualitative behaviour of the spread as a function of time is very different from that of a market making agent. Namely, the spreads at each inventory level do not approach asymptotic values when the time to maturity is large. The intuitive explanation for this lies in the fact that the liquidating agent is only able to make a finite number of trades, but the market maker does not have a bound on the number of trades they may execute. This means that for the market maker, when the time to maturity is large, they seek a balance between executing trades at a high rate and executing trades at largely profitable prices. The liquidating agent achieves no benefit from selling shares quickly when the time to maturity is large, and so they simply elect to post large spreads in order to possibly benefit from a small number of highly profitable trades. This behaviour changes as time approaches maturity where posting smaller spreads is optimal since they would prefer to sell shares through the use of limit orders rather than suffer the terminal liquidation penalty. This effect is so strong for large inventory approaching maturity that the agent prefers to essentially give away inventory at no premium rather than risk paying this penalty. This is indicated in Figure 3.1 by the spreads that are equal to zero.

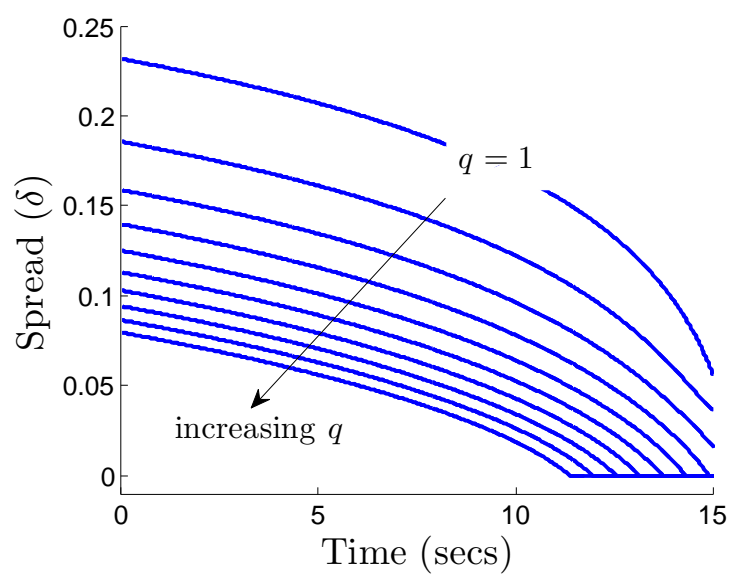

Figure 3.1: Optimal spread for an ambiguity neutral agent. Parameter values are $\kappa=15$, $\lambda=2, \sigma=0.01, \alpha=0, \ell(q)=\theta q, \theta=0.01, Q=10$ and $T=15$ seconds.

\subsection{Ambiguity Aversion}

The different posting behaviour within the reference model of the liquidating agent compared to the market maker begs the question as to whether the effects of ambiguity 
aversion will have similar effects on the spreads. This is the topic that is to be explored in this section. The method in which the agent imposes ambiguity aversion is equivalent to that of the market maker in Section 2.2. The agent selects a factor of the model which they declare to be misspecified, and then introduces a class of equivalent measures under which that factor may take on any value. A penalization term added to the optimization problem quantifies the level of ambiguity aversion that the agent has towards that factor of the reference model. Once the appropriate class of measures has been selected, the optimization problem becomes:

$$
H(t, x, q, S ; \varphi)=\sup _{\left(\delta_{u}\right)_{t \leq u \leq T} \in \mathcal{A}} \inf _{\mathbb{Q} \in \mathcal{Q}} \mathbb{E}_{t, x, q, S}^{\mathbb{Q}}\left[X_{\tau \wedge T}+q_{\tau \wedge T}\left(S_{\tau \wedge T}-\ell\left(q_{\tau \wedge T}\right)\right)+\mathcal{H}_{t, \tau \wedge T}(\mathbb{Q} \mid \mathbb{P})\right]
$$

where

$$
\mathcal{H}_{t, \tau \wedge T}(\mathbb{Q} \mid \mathbb{P})=\frac{1}{\varphi} \log \left\{\left(\frac{d \mathbb{Q}}{d \mathbb{P}}\right)_{\tau \wedge T} /\left(\frac{d \mathbb{Q}}{d \mathbb{P}}\right)_{t}\right\}
$$

The resulting HJB equation to be solved is of the form:

$$
\partial_{t} H+\sup _{\delta \geq 0} \inf _{\mathbb{Q} \in \mathcal{Q}}\left\{\mathcal{L}^{\mathbb{Q}} H-\partial_{t} \mathbb{E}_{t, x, q, S}^{\mathbb{Q}}\left[\mathcal{H}_{t, T}[\mathbb{Q} \mid \mathbb{P}]\right]\right\}=0
$$

For the remainder of this section, the full HJB equation for $H(t, x, q, S)$ will not be displayed, rather the equations associated with the ansatz $h_{q}(t)$ will be given, as they offer a more easily written form of the feedback controls.

\subsubsection{Ambiguity aversion with respect to market order arrivals}

When considering ambiguity with respect to market order arrivals, the measure changes are defined by:

$$
\left(\frac{d \mathbb{Q}(g)}{d \mathbb{P}}\right)=\exp \left\{-\int_{0}^{T} \int_{0}^{\infty}\left(e^{g_{t}}-1\right) \nu_{\mathbb{P}}(d y, d t)+\int_{0}^{T} \int_{0}^{\infty} g_{t} \mu(d y, d t)\right\},
$$

where the set of candidate measures belong to:

$$
\mathcal{Q}^{\lambda}=\left\{\mathbb{Q}(g): g_{t} \text { are } \mathcal{F}_{t} \text {-predictable, Markov, and bounded }\right\}
$$


The infinitesimal generator $\mathcal{L}^{\mathbb{Q}(g)}$ is given by:

$$
\mathcal{L}^{\mathbb{Q}(g)} H^{\lambda}\left(t, x, q, S ; \varphi_{\lambda}\right)=\alpha \partial_{S} H^{\lambda}+\frac{1}{2} \sigma^{2} \partial_{S S} H^{\lambda}+\lambda e^{g_{t}} e^{-\kappa \delta} \mathcal{D} H^{\lambda} \mathbb{1}_{q \neq 0}
$$

The corresponding system of ODEs for the ansatz function $h_{q}^{\varphi_{\lambda}}(t)$ is:

$$
\begin{aligned}
\partial_{t} h_{q}^{\varphi_{\lambda}}+\alpha q+\sup _{\delta \geq 0} \inf _{g}\left\{\lambda e^{g} e^{-\kappa \delta}\left(\delta+h_{q-1}^{\varphi_{\lambda}}-h_{q}^{\varphi_{\lambda}}\right)+\frac{1}{\varphi_{\lambda}} \lambda\left(e^{g}(g-1)+1\right)\right\} & =0,(3.10) \\
h_{q}^{\varphi_{\lambda}}(T) & =-q \ell(q) . \\
h_{0}^{\varphi_{\lambda}}(t) & =0
\end{aligned}
$$

Proposition 13 (Optimal Feedback Controls). The optimal feedback controls of equation (3.10) are given by

$$
\begin{aligned}
& \delta^{*}\left(t, q ; \varphi_{\lambda}\right)=\left(\frac{1}{\kappa}-h_{q-1}^{\varphi_{\lambda}}(t)+h_{q}^{\varphi_{\lambda}}(t)\right)_{+}, q \neq 0, \\
& g^{*}\left(t, q ; \varphi_{\lambda}\right)=-\varphi_{\lambda} e^{-\kappa \delta^{*}\left(t, q ; \varphi_{\lambda}\right)}\left(\delta^{*}\left(t, q ; \varphi_{\lambda}\right)+h_{q-1}^{\varphi_{\lambda}}(t)-h_{q}^{\varphi_{\lambda}}(t)\right) .
\end{aligned}
$$

Proof. Apply first order conditions in $g$ then $\delta$ to achieve the results. The computations involved are equivalent to those in Proposition 2.

\section{Discussion and Financial Interpretation}

Substituting the expression for $g^{*}\left(t, q ; \varphi_{\lambda}\right)$ into equation (3.10) results in an equation which can easily be solved numerically:

$$
\partial_{t} h_{q}^{\varphi_{\lambda}}+\alpha q+\sup _{\delta \geq 0}\left\{\frac{\lambda}{\varphi_{\lambda}}\left(1-e^{-\varphi_{\lambda} e^{-\kappa \delta}\left(\delta+h_{q-1}^{\varphi_{\lambda}}-h_{q}^{\varphi_{\lambda}}\right)}\right)\right\} \mathbb{1}_{q \neq 0}=0
$$

Solving for $h_{q}^{\varphi_{\lambda}}(t)$ and computing the optimal spreads allows for a comparison to the spreads corresponding to the reference model as shown in Figure 3.2. Of particular interest is how different the effective market order intensities can be depending on the remaining time to maturity. For a fixed level of inventory, the effective market order intensity decreases as maturity approaches because that is the time in which such a change can impair the agent's performance the most (however the effective market order rate can not decrease below $\left.\lambda e^{-\frac{\varphi_{\lambda}}{\kappa}}\right)$. Also note that the largest change in the optimal 

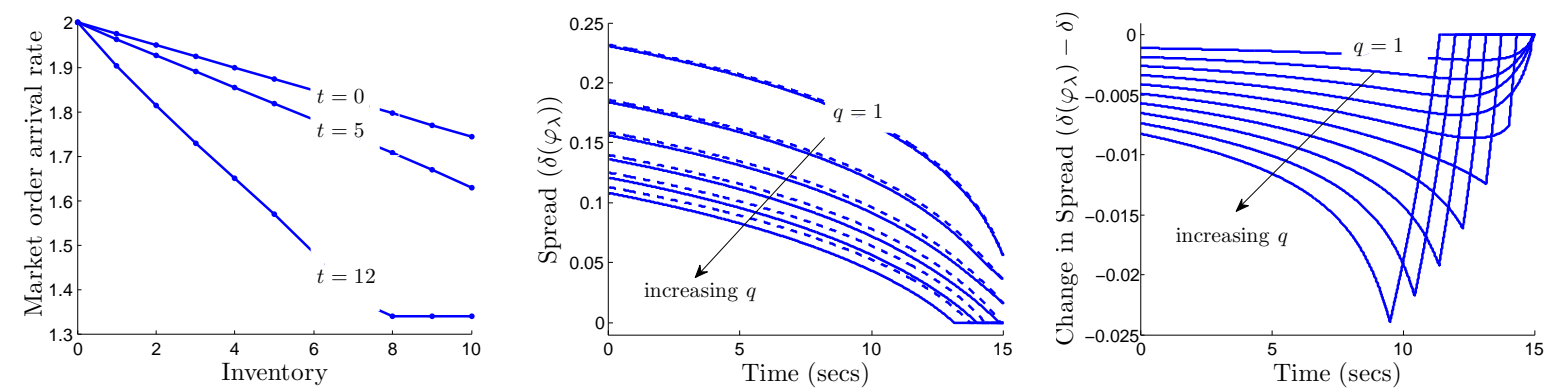

Figure 3.2: Market order arrival rate induced by optimal $g_{t}$, optimal spread, and change in spread due to ambiguity for an agent who is ambiguity averse to MO rate of arrival (dashed lines are ambiguity neutral spreads). Parameter values are $\varphi_{\lambda}=6, \kappa=15$, $\lambda=2, \sigma=0.01, \alpha=0, \ell(q)=\theta q, \theta=0.01, Q=10$ and $T=15$.

posting occurs for the largest value of inventory. When the inventory is at its maximum, this is when the agent's fear of a misspecified arrival rate can have most significant impact on their trading performance.

\subsubsection{Ambiguity aversion with respect to fill probability}

The appropriate set of measure changes to enforce ambiguity on the fill probability are:

$$
\left(\frac{d \mathbb{Q}(g)}{d \mathbb{P}}\right)=\exp \left\{-\int_{0}^{T} \int_{0}^{\infty}\left(e^{g_{t}(y)}-1\right) \nu_{\mathbb{P}}(d y, d t)+\int_{0}^{T} \int_{0}^{\infty} g_{t}(y) \mu(d y, d t)\right\},
$$

the candidate measures are:

$$
\begin{gathered}
\mathcal{Q}^{\kappa}=\left\{\mathbb{Q}(g): g_{t}(y) \text { are } \mathcal{F}_{t^{-} \text {-predictable, Markov, and bounded, }}\right. \\
\text { and satisfy } \left.\int_{0}^{\infty} e^{g_{t}(y)} F(y) d y=1 \text { for all } t \in[0, T]\right\} .
\end{gathered}
$$

The infinitesimal generator $\mathcal{L}^{\mathbb{Q}(g)}$ is given by:

$$
\mathcal{L}^{\mathbb{Q}(g)} H^{\kappa}\left(t, x, q, S ; \varphi_{\kappa}\right)=\alpha \partial_{S} H^{\kappa}+\frac{1}{2} \sigma^{2} \partial_{S S} H^{\kappa}+\lambda \int_{\delta}^{\infty} e^{g_{t}(y)} F(y) d y \mathcal{D} H^{\kappa} \mathbb{1}_{q \neq 0}
$$


This results in a system of ODE's for the ansatz function $h_{q}^{\varphi_{\kappa}}(t)$ of the following form:

$$
\begin{aligned}
\partial_{t} h_{q}^{\varphi_{\kappa}}+\alpha q+\sup _{\delta \geq 0} \inf _{g \in \mathcal{G}}\left\{\lambda\left[\int_{\delta}^{\infty} e^{g(y)} F(y) d y\right]\left(\delta+h_{q-1}^{\varphi_{\kappa}}-h_{q}^{\varphi_{\kappa}}\right)\right. & \\
\left.+\frac{1}{\varphi_{\kappa}} \lambda \int_{0}^{\infty}\left(e^{g(y)}(g(y)-1)+1\right) F(y) d y\right\} & =0, \\
h_{q}^{\varphi_{\kappa}}(T) & =-q \ell(q) . \\
h_{0}^{\varphi_{\kappa}}(t) & =0
\end{aligned}
$$

where the class of functions:

$$
\mathcal{G}=\left\{g: \int_{0}^{\infty} e^{g(y)} F(y) d y=1\right\} .
$$

Proposition 14 (Optimal Feedback Controls). The optimal feedback controls of equation (3.12) are given by

$$
\begin{aligned}
\delta^{*}\left(t, q ; \varphi_{\kappa}\right) & =\left(\frac{1}{\varphi_{\kappa}} \log \left(1+\frac{\varphi_{\kappa}}{\kappa}\right)-h_{q-1}^{\varphi_{\kappa}}(t)+h_{q}^{\varphi_{\kappa}}(t)\right)_{+}, q \neq 0 \\
g^{*}\left(t, q, y ; \varphi_{\kappa}\right)= & -\log \left(1-e^{-\kappa \delta^{*}\left(t, q ; \varphi_{\kappa}\right)}\left(1-e^{-\varphi_{\kappa}\left(\delta^{*}\left(t, q ; \varphi_{\kappa}\right)+h_{q-1}^{\varphi_{\kappa}}(t)-h_{q}^{\varphi_{\kappa}}(t)\right)}\right)\right) \\
& \quad-\varphi_{\kappa}\left(\delta^{*}\left(t, q ; \varphi_{\kappa}\right)+h_{q-1}^{\varphi_{\kappa}}(t)-h_{q}^{\varphi_{\kappa}}(t)\right) \mathbb{1}_{y \geq \delta^{*}\left(t, q ; \varphi_{\kappa}\right)} .
\end{aligned}
$$

Proof. These quantities are computed in an identical manner to those of Proposition 3.

\section{Discussion and Financial Interpretation}

Substituting the expression for $g^{*}\left(t, q, y ; \varphi_{\kappa}\right)$ into equation (3.12) gives an equation for $h_{q}^{\varphi_{\kappa}}(t)$ that is easily solved numerically:

$$
\partial_{t} h_{q}^{\varphi_{\kappa}}+\alpha q+\sup _{\delta \geq 0}\left\{\frac{-\lambda}{\varphi_{\kappa}} \log \left(1-e^{-\kappa \delta}+e^{-\kappa \delta-\varphi_{\kappa}\left(\delta+h_{q-1}^{\varphi_{\kappa}}-h_{q}^{\varphi_{\kappa}}\right)}\right)\right\} \mathbb{1}_{q \neq 0}=0
$$

The change in optimal posting due to ambiguity on the fill probability, shown in Figure 3.3, is qualitatively different than in the case of market order intensity. First, note that the change is smallest when the inventory position is largest. This is because the agent 

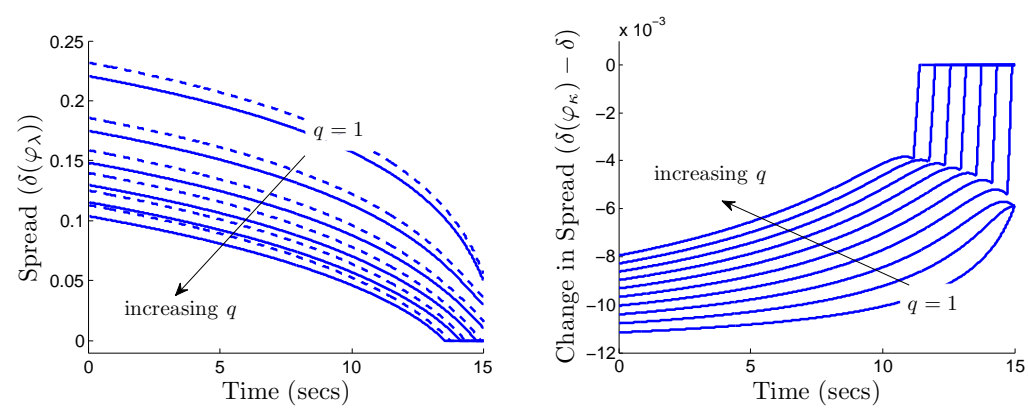

Figure 3.3: Optimal spread, and change in spread due to ambiguity for an agent who is ambiguity averse to fill probability (dashed lines are ambiguity neutral spreads). Parameter values are $\varphi_{\kappa}=3, \kappa=15, \lambda=2, \sigma=0.01, \alpha=0, \ell(q)=\theta q, \theta=0.01, Q=10$ and $T=15$.

naturally protects themselves against ambiguity aversion when inventory levels are large by posting small spreads. When comparing a small spread to a large spread, an equal change in the fill probability suffers a larger penalty for the small spread. Second, the magnitude of the changes increase with time to maturity rather than decrease.

\subsubsection{Ambiguity aversion with respect to midprice drift}

The appropriate form of measure change to account for ambiguity in the midprice drift is:

$$
\left(\frac{d \mathbb{Q}(\eta)}{d \mathbb{P}}\right)=\exp \left\{-\frac{1}{2} \int_{0}^{T}\left(\frac{\alpha-\eta_{t}}{\sigma}\right)^{2} d t-\int_{0}^{T} \frac{\alpha-\eta_{t}}{\sigma} d W_{t}\right\}
$$

where the class of measures to be considered is restricted to the set:

$$
\mathcal{Q}^{\alpha}=\left\{\mathbb{Q}^{\alpha}(\eta): \eta \text { is } \mathcal{F}_{t} \text {-predictable, Markov, and bounded }\right\} .
$$

The infinitesimal generator $\mathcal{L}^{\mathbb{Q}(\eta)}$ is given by:

$$
\mathcal{L}^{\mathbb{Q}(\eta)} H^{\alpha}\left(t, x, q, S ; \varphi_{\alpha}\right)=\eta_{t} \partial_{S} H^{\alpha}+\frac{1}{2} \sigma^{2} \partial_{S S} H^{\alpha}+\lambda e^{-\kappa \delta} \mathcal{D} H^{\alpha} \mathbb{1}_{q \neq 0}
$$


The system of ODE's which results from the corresponding penalty function is:

$$
\begin{aligned}
\partial_{t} h_{q}^{\varphi_{\alpha}}+\sup _{\delta \geq 0} \inf _{\eta}\left\{\lambda e^{-\kappa \delta}\left(\delta+h_{q-1}^{\varphi_{\alpha}}-h_{q}^{\varphi_{\alpha}}\right)+\eta q+\frac{1}{2 \varphi_{\alpha}}\left(\frac{\alpha-\eta}{\sigma}\right)^{2}\right\} & =0 \\
h_{q}^{\varphi_{\alpha}}(T) & =-q \ell(q) \\
h_{0}^{\varphi_{\alpha}}(t) & =0
\end{aligned}
$$

Proposition 15 (Optimal Feedback Controls). The optimal feedback controls of the HJB equation (3.15) are given by

$$
\begin{aligned}
& \delta^{*}\left(t, q ; \varphi_{\alpha}\right)=\left(\frac{1}{\kappa}-h_{q-1}^{\varphi_{\alpha}}(t)+h_{q}^{\varphi_{\alpha}}(t)\right)_{+}, \quad q \neq 0, \\
& \eta^{*}\left(t, q ; \varphi_{\alpha}\right)=\alpha-\varphi_{\alpha} \sigma^{2} q .
\end{aligned}
$$

Proof. Apply first order conditions to the supremum and infimum terms in equation (3.15).

\section{Discussion and Financial Interpretation}

Substituting $\eta^{*}\left(t, q ; \varphi_{\alpha}\right)$ into equation (3.15) gives the following equation to be solved numerically:

$$
\partial_{t} h_{q}^{\varphi_{\alpha}}+\alpha q-\frac{1}{2} \varphi_{\alpha} \sigma^{2} q^{2}+\sup _{\delta \geq 0}\left\{\lambda e^{-\kappa \delta}\left(\delta+h_{q-1}^{\varphi_{\alpha}}-h_{q}^{\varphi_{\alpha}}\right)\right\} \mathbb{1}_{q \neq 0}=0
$$
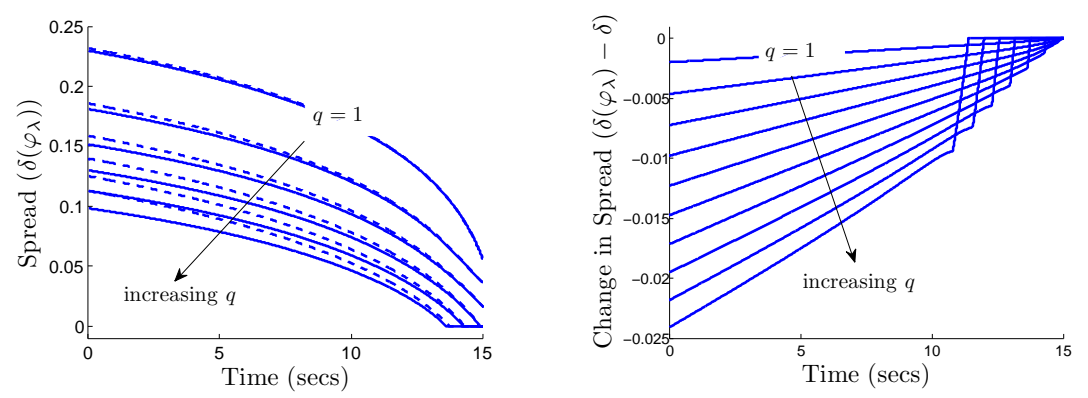

Figure 3.4: Optimal spread, and change in spread due to ambiguity for an agent who is ambiguity averse to midprice drift (dashed lines are ambiguity neutral spreads). Parameter values are $\varphi_{\alpha}=5, \kappa=15, \lambda=2, \sigma=0.01, \alpha=0, \ell(q)=\theta q, \theta=0.01, Q=10$ and $T=15$. 


\section{Equivalence to Inventory Penalization}

Once again, consider an agent with the goal of liquidating a number of shares before some time $T$, but without the consideration of ambiguity aversion. Instead, the agent will enforce a quadratic inventory penalization so that their value function is:

$$
H^{\phi}(t, x, q, S)=\sup _{\left(\delta_{u}\right)_{t \leq u \leq T} \in \mathcal{A}} \mathbb{E}_{t, x, q, S}^{\mathbb{P}}\left[X_{\tau \wedge T}+q_{\tau \wedge T}\left(S_{\tau \wedge T}-\ell\left(q_{\tau \wedge T}\right)\right)-\frac{1}{2} \phi \sigma^{2} \int_{t}^{\tau \wedge T} q_{u}^{2} d u\right]
$$

After the usual ansatz, substitution into the corresponding HJB equation, and solving for the optimal controls $\delta$, the resulting system of ODE's is:

$$
\partial_{t} h_{q}^{\phi}+\alpha q-\frac{1}{2} \phi \sigma^{2} q^{2}+\sup _{\delta \geq 0}\left\{\lambda e^{-\kappa \delta}\left(\delta+h_{q-1}^{\phi}-h_{q}^{\phi}\right)\right\} \mathbb{1}_{q \neq 0}=0
$$

In the same fashion as the market making problem, this equation is seen to be equivalent to equation (3.16), and so the two value functions are also equal:

$$
\begin{array}{r}
\sup _{\left(\delta_{u}\right)_{t \leq u \leq T} \in \mathcal{A}} \mathbb{E}_{t, x, q, S}^{\mathbb{P}}\left[X_{\tau \wedge T}+q_{\tau \wedge T}\left(S_{\tau \wedge T}-\ell\left(q_{\tau \wedge T}\right)\right)-\frac{1}{2} \phi \sigma^{2} \int_{t}^{\tau \wedge T} q_{u}^{2} d u\right] \\
=\sup _{\left(\delta_{u}\right)_{t \leq u \leq T} \in \mathcal{A}} \inf _{\mathbb{Q} \in \mathcal{Q}^{\alpha}} \mathbb{E}_{t, x, q, S}^{\mathbb{Q}}\left[X_{\tau \wedge T}+q_{\tau \wedge T}\left(S_{\tau \wedge T}-\ell\left(q_{\tau \wedge T}\right)\right)+\mathcal{H}_{t, \tau \wedge T}[\mathbb{Q} \mid \mathbb{P}]\right]
\end{array}
$$

Once again, a cumulative inventory penalization is seen to be equivalent to considering ambiguity on the drift of the midprice of the asset.

As shown in Figure 3.4, the change in the optimal spread is smallest when inventory is smallest. However, the change in optimal spread consistently becomes more negative with time to maturity. Both of these behaviours make sense when midprice ambiguity is interpreted as a cumulative inventory penalization. The first characteristic is made clear by noting that the larger the agent's inventory position is, the larger the accumulation of the inventory penalty is, and the faster they desire to liquidate shares. The second characteristic is explained by noting that the impact on the agent's performance due to a misspecified drift is approximately linear in time to maturity.

The effect of ambiguity on the drift of the midprice will turn out to be more significant than ambiguity on the other two factors in the case of the liquidation problem. As shown in Section 3.3, specifically in Proposition 18, the optimal limit order price grows without bound as the time to maturity increases when there is no ambiguity on drift. However, 
when ambiguity on drift is considered, all of the optimal posting levels become finite for all time.

\subsubsection{Mixed Ambiguity}

Just as the market maker may experience ambiguity aversion from several sources, so too may the liquidating agent. Fortunately, the case of mixed ambiguity is handled in an equivalent manner by performing the change of measure as a sequence of three measure changes, each one appropriately penalized. For the sake of brevity, the details of the measure changes and penalty function are not repeated as they are identical to what is discussed in Section 2.3. The value function for the agent in this case is still given by equation (3.7), and the penalty is selected as:

$$
\mathcal{H}\left(\mathbb{Q}^{\alpha, \lambda, \kappa}(\eta, g) \mid \mathbb{P}\right)=\mathcal{H}^{\alpha}\left(\mathbb{Q}^{\alpha}(\eta) \mid \mathbb{P}\right)+\mathcal{H}^{\lambda, \kappa}\left(\mathbb{Q}^{\alpha, \lambda, \kappa}(\eta, g) \mid \mathbb{Q}^{\alpha}(\eta)\right)
$$

where:

$$
\mathcal{H}^{\lambda, \kappa}\left(\mathbb{Q}^{\alpha, \lambda, \kappa}(\eta, g) \mid \mathbb{Q}^{\alpha}(\eta)\right)= \begin{cases}\frac{1}{\varphi_{\lambda}} \log \left(\frac{d \mathbb{Q}^{\alpha, \lambda}\left(\eta, g^{\lambda}\right)}{d \mathbb{Q}^{\alpha}(\eta)}\right)+\frac{1}{\varphi_{\kappa}} \log \left(\frac{d \mathbb{Q}^{\alpha, \lambda, \kappa}(\eta, g)}{d \mathbb{Q}^{\alpha, \lambda}\left(\eta, g^{\lambda}\right)}\right), & \text { if } \varphi_{\lambda} \geq \varphi_{\kappa} \\ \frac{1}{\varphi_{\kappa}} \log \left(\frac{d \mathbb{Q}^{\alpha, \kappa}\left(\eta, g^{\kappa}\right)}{d \mathbb{Q}^{\alpha}(\eta)}\right)+\frac{1}{\varphi_{\lambda}} \log \left(\frac{d \mathbb{Q}^{\alpha, \lambda, \kappa}(\eta, g)}{d \mathbb{Q}^{\alpha, \kappa}\left(\eta, g^{\kappa}\right)}\right), & \text { if } \varphi_{\lambda} \leq \varphi_{\kappa}\end{cases}
$$

A skip is made directly to the resulting system of ODEs in the ansatz function. Denoting the triple of ambiguity levels by $\psi=\left(\varphi_{\alpha}, \varphi_{\lambda}, \varphi_{\kappa}\right)$, and reintroducing the functional $\mathcal{K}^{c, d}(a, b)$, the ansatz function $h_{q}^{\psi}(t)$ satisfies:

$$
\begin{aligned}
& \partial_{t} h_{q}^{\psi}+\inf _{\eta}\left\{\eta q+\frac{1}{2 \varphi_{\alpha}}\left(\frac{\alpha-\eta}{\sigma}\right)^{2}\right\} \\
&+\sup _{\delta \geq 0} \inf _{g^{\lambda}} \inf _{g^{\kappa} \in \mathcal{G}}\left\{\lambda\left[\int_{\delta}^{\infty} e^{g_{t}^{\lambda}+g_{t}^{\kappa}(y)} F(y) d y\right]\left(\delta+h_{q-1}^{\psi}-h_{q}^{\psi}\right)\right. \\
&\left.+\mathcal{K}^{\varphi_{\lambda}, \varphi_{\kappa}}\left(g_{t}^{\lambda}, g_{t}^{\kappa}\right) \mathbb{1}_{\varphi_{\lambda} \geq \varphi_{\kappa}}+\mathcal{K}^{\varphi_{\kappa}, \varphi_{\lambda}}\left(g_{t}^{\kappa}, g_{t}^{\lambda}\right) \mathbb{1}_{\varphi_{\lambda}<\varphi_{\kappa}}\right\}=0 . \\
& h_{q}^{\psi}(T)=-q \ell(q) \\
& h_{0}^{\psi}(t)=0
\end{aligned}
$$


where

$$
\begin{aligned}
\mathcal{K}^{c, d}(a, b)= & \frac{1}{c} \int_{0}^{\infty}\left[-\left(e^{a(y)}-1\right)+a(y) e^{a(y)+b(y)}\right] \lambda F(y) d y \\
& +\frac{1}{d} \int_{0}^{\infty}\left[-\left(e^{b(y)}-1\right) e^{a(y)}+b(y) e^{a(y)+b(y)}\right] \lambda F(y) d y .
\end{aligned}
$$

Proposition 16 (Optimal Feedback Controls). The optimal feedback controls of equation (3.17) are given by:

$$
\begin{aligned}
\delta^{*}(t, q ; \psi) & =\left(\frac{1}{\varphi_{\kappa}} \log \left(1+\frac{\varphi_{\kappa}}{\kappa}\right)-h_{q-1}^{\psi}(t)+h_{q}^{\psi}(t)\right)_{+}, q \neq 0, \\
g^{\lambda *}(t, q ; \psi)= & \frac{\varphi_{\lambda}}{\varphi_{\kappa}} \log \left(1-e^{-\kappa \delta^{*}(t, q ; \psi)}\left(1-e^{-\varphi_{\kappa}\left(\delta^{*}(t, q ; \psi)+h_{q-1}^{\psi}(t)-h_{q}^{\psi}(t)\right)}\right)\right), \\
g^{*}(t, q, y ; \psi)= & -\log \left(1-e^{-\kappa \delta^{*}(t, q ; \psi)}\left(1-e^{-\varphi_{\kappa}\left(\delta^{*}(t, q ; \psi)+h_{q-1}^{\psi}(t)-h_{q}^{\psi}(t)\right)}\right)\right) \\
\quad & \quad-\varphi_{\kappa}\left(\delta^{*}(t, q ; \psi)+h_{q-1}^{\psi}(t)-h_{q}^{\psi}(t)\right) \mathbb{1}_{y \geq \delta^{*}\left(t, q ; \varphi_{\kappa}\right)} . \\
\eta^{*}(t, q ; \psi)= & \alpha-\varphi_{\alpha} \sigma^{2} q .
\end{aligned}
$$

Proof. The computations that yield these optimal values are equivalent to those of Proposition 7 .

In Figure 3.5 it can be seen that the way in which the liquidating agent alters their spreads to account for mixed ambiguity certainly depends on the relative sizes of each of the three ambiguity levels. Aspects of the modification to consider are whether the spreads should change more for large or small inventory, and whether the magnitude of the change should grow with time to maturity or become stable. With mixed ambiguity, there is no single rule by which the agent changes their spreads that properly accounts for all of their types of aversion.

\subsection{Closed Form Solutions}

In the market making problem, there is a special case in which closed form analytical solutions exist for the value function, and therefore also the optimal spreads. A similar situation holds for the liquidating agent, but the form of these solutions is quite different. 

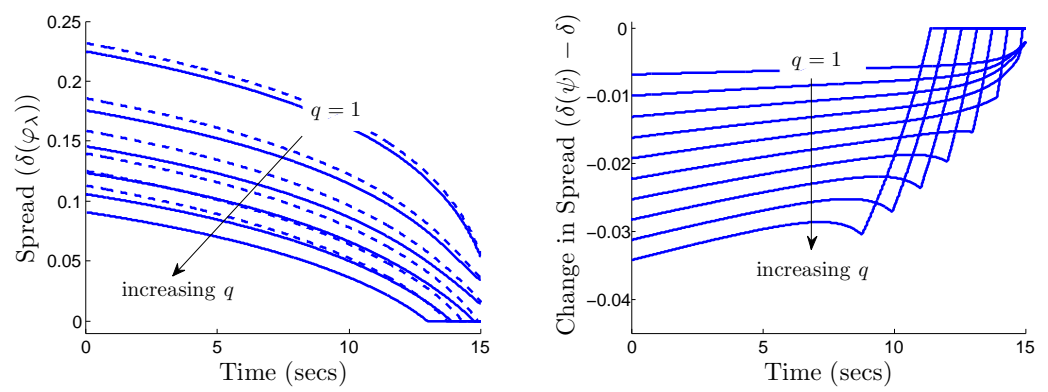

(a) ambiguity levels: $\varphi_{\alpha}=5, \varphi_{\lambda}=5, \varphi_{\kappa}=1$
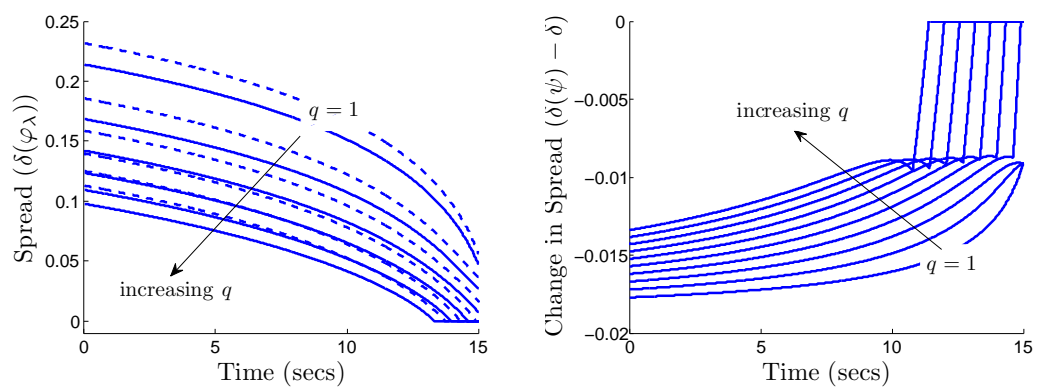

(b) ambiguity levels: $\varphi_{\alpha}=0, \varphi_{\lambda}=1, \varphi_{\kappa}=5$

Figure 3.5: Optimal sell and total spreads for an MM who utilizes mixed ambiguity (dashed lines are ambiguity neutral spreads). Parameter values are $\kappa=15, \lambda=2$, $\sigma=0.01, \alpha=0, \ell(q)=\theta q, \theta=0.01, Q=10$ and $T=15$. 
Suppose the agent has equal levels of ambiguity to MO arrival rate and fill probabilities so that $\varphi_{\lambda}=\varphi_{\kappa}=\varphi$. Suppose further that the optimal spreads $\delta^{*}(t, q ; \psi)$ given by Proposition 16 are positive. Then substituting all of the optimal controls into equation (3.17) gives:

$$
\partial_{t} h_{q}^{\psi}+\alpha q-\frac{1}{2} \varphi_{\alpha} \sigma^{2} q^{2}+\frac{\xi}{\kappa} e^{-\kappa\left(-h_{q-1}^{\psi}+h_{q}^{\psi}\right)}=0
$$

where

$$
\xi=\left(1+\frac{\varphi}{\kappa}\right)^{-\left(1+\frac{\kappa}{\varphi}\right)} \lambda
$$

with terminal and boundary conditions $h_{q}^{\psi}(T)=-q \ell(q)$ and $h_{0}^{\psi}(t)=0$.

Proposition 17 (Solving for $h_{q}^{\psi}(t)$ in equation (3.18)). Let $K_{q}=\alpha \kappa q-\frac{1}{2} \varphi_{\alpha} \sigma^{2} \kappa q^{2}$.

i) Suppose $K_{q}=0$ for all $q$ (that is, $\varphi_{\alpha}=0$ and $\alpha=0$ ). Then:

$$
h_{q}^{\psi}(t)=\frac{1}{\kappa} \log \left(\sum_{n=0}^{q} C_{q, n}(T-t)^{n}\right)
$$

where

$$
C_{q, n}=\frac{\xi^{n}}{n !} e^{-\kappa(q-n) \ell(q-n)}
$$

ii) Suppose $K_{q}=0$ only when $q=0$. Then:

$$
h_{q}^{\psi}(t)=\frac{1}{\kappa} \log \left(\sum_{n=0}^{q} C_{q, n} e^{K_{n}(T-t)}\right)
$$

where

$$
\begin{aligned}
C_{n+j, n} & =(-\xi)^{j} \prod_{p=1}^{j} \frac{1}{K_{n+p}-K_{n}} C_{n, n} \\
C_{q, q} & =-\sum_{n=1}^{q-1} C_{q, n}+e^{-\kappa q \ell(q)} \\
C_{0,0} & =1
\end{aligned}
$$


Proof. See Appendix B.2.

The fact that proposition 17 shows that the value function can have two completely different functional forms depending on the possible values of $K_{q}=\alpha \kappa q-\frac{1}{2} \varphi_{\alpha} \sigma^{2} \kappa q^{2}$ begs the question as to what effect this has on the optimal spread $\delta^{*}(t, q ; \psi)$. Indeed, the behaviour of the spreads for case i) $\left(K_{q}=0, \forall q>0\right)$ and a more specific case ii) $\left(K_{q}<0, \forall q>0\right)$ are quite different as the time to maturity gets very large:

Proposition 18 (Behaviour of optimal spreads as $(T-t) \rightarrow \infty$ ). Let $\tau=(T-t)$. In case i) of Proposition 17, the optimal spreads $\delta^{*}(t, q ; \psi)$ grow as $\frac{1}{\kappa} \log \left(\frac{\xi}{q} \tau\right)$ as $\tau \rightarrow \infty$. In case ii) of Proposition 17, the optimal spreads $\delta^{*}(t, q ; \psi)$ approach $\frac{1}{\varphi_{\kappa}} \log \left(1+\frac{\varphi_{\kappa}}{\kappa}\right)+$ $\frac{1}{\kappa} \log \left(\frac{-\xi}{K_{q}}\right)$ as $\tau \rightarrow \infty$.

Proof. See Appendix B.2.

\subsection{Inclusion of Market Orders}

Up to this point, both the market maker and the liquidating agent have been restricted to trades using limit orders only. However, there is a possibility that they improve their trading performance if they are also allowed to execute market orders. This allowance is more natural in the setting of a liquidation problem, as a market maker is generally thought of as an agent that provides liquidity to the market rather than taking it. Mathematically, the inclusion of market orders corresponds to the additional use of an impulse control by the agent.

The effect of market orders on the agent's inventory and wealth dynamics will be treated as follows: all market sell orders executed by the agent will be at a price $\frac{\Delta}{2}$ less than the midprice. Denote the number of market orders executed by the agent up to time $t$ by $J_{t}$. 
Then the updated dynamics of the agent's inventory and wealth are:

$$
\begin{aligned}
d q_{t} & =-d N_{t}-d J_{t} \\
d X_{t} & =\left(S_{t}+\delta_{t}\right) d N_{t}+\left(S_{t}-\frac{\Delta}{2}\right) d J_{t} .
\end{aligned}
$$

The agent's additional control is the support of the object $d J_{t}$.

\subsubsection{Feedback controls}

The agent must select a sequence of stopping times $\tau_{k}$ at which they execute a market order. The updated optimization problem for the ambiguity neutral agent is:

$$
H(t, x, q, S)=\sup _{\substack{\left(\delta_{s}\right)_{t \leq s \leq T} \in \mathcal{A} \\\left(\tau_{k}\right)_{k=1, \ldots, Q}}} \mathbb{E}_{t, x, q, S}^{\mathbb{P}}\left[X_{\tau \wedge T}+q_{\tau \wedge T}\left(S_{\tau \wedge T}-\ell\left(q_{\tau \wedge T}\right)\right)\right]
$$

The inclusion of market orders changes the equation satisfied by the value function $H(t, x, q, S)$. Rather than a standard HJB equation, $H$ now satisfies a quasi-variational inequality of the following form:

$$
\begin{aligned}
\max \left\{\left(\partial_{t} H+\alpha \partial_{S} H+\frac{1}{2} \sigma^{2} \partial_{S S} H+\sup _{\delta \geq 0}\left\{\lambda e^{-\kappa \delta} \mathcal{D} H\right\} \mathbb{1}_{q \neq 0}\right),\right. & \\
\left.\left(H\left(t, x+\left(S-\frac{\Delta}{2}\right), q-1, S\right)-H(t, x, q, S)\right)\right\} & =0,
\end{aligned}
$$

From equation (3.19), it is clear that one of the two terms must be equal to zero, and the other term must be less than or equal to zero. This allows the definition of a continuation region and execution region:

$$
\begin{aligned}
& \mathcal{C}=\left\{(t, x, q, S): \partial_{t} H+\alpha \partial_{S} H+\frac{1}{2} \sigma^{2} \partial_{S S} H+\sup _{\delta}\left\{\lambda e^{-\kappa \delta} \mathcal{D} H\right\} \mathbb{1}_{q \neq 0}=0\right\}, \\
& \mathcal{E}=\left\{(t, x, q, S): H\left(t, x+\left(S-\frac{\Delta}{2}\right), q-1, S\right)-H(t, x, q, S)=0\right\} .
\end{aligned}
$$

Whenever $(t, x, q, S) \in \mathcal{E}$, it is beneficial for the agent to execute a market order. When $(t, x, q, S) \in \mathcal{C}$, it is more beneficial for the agent to refrain from executing a market order and continue with the optimal placement of limit orders. Whereas in the case of using only limit orders, it was the optimal spreads $\delta_{t}$ that were of interest, in this case the 
boundary between the continuation and execution regions is also of interest. The usual ansatz $H(t, x, q, S)=x+q S+h_{q}(t)$ still applies which simplifies the quasi-variational inequality to:

$$
\max \left\{\left(\partial_{t} h+\alpha q+\sup _{\delta \geq 0}\left\{\lambda e^{-\kappa \delta}\left(\delta+h_{q-1}-h_{q}\right)\right\} \mathbb{1}_{q \neq 0}\right),\left(h_{q-1}-h_{q}-\frac{\Delta}{2}\right)\right\}=0,
$$

It is clear that the feedback expression for the optimal spreads are of the same form as in Proposition 12. However, the numerical values of these spreads will be different because the values of the functions $h_{q}(t)$ will be different. Also of importance is to note that after making the ansatz, the continuation and execution regions can be redefined in terms of $h_{q}(t)$ and therefore will not depend on the state variables $x$ and $S$. The boundary between the two regions will therefore be a curve in the $(t, q)$ plane. In Figure
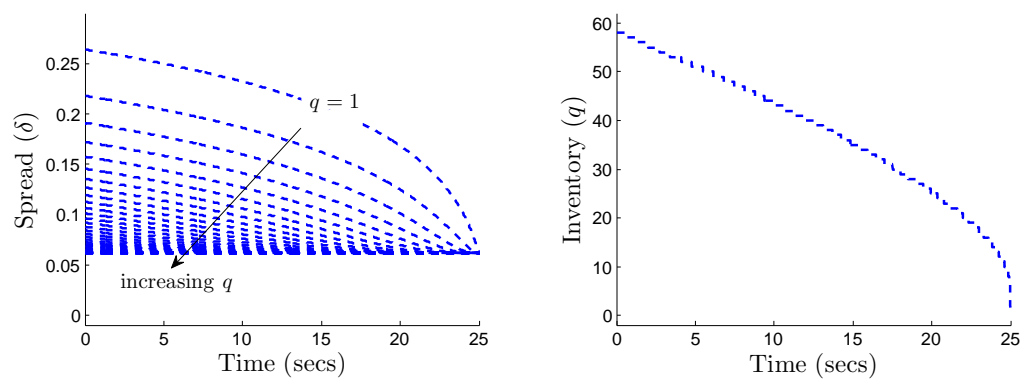

Figure 3.6: Optimal spread, and market order execution boundary for ambiguity neutral agent. Parameter values are $\kappa=15, \lambda=2, \sigma=0.01, \alpha=0, \ell(q)=\theta q, \theta=0.01$, $\Delta=0.01, Q=60$ and $T=25$.

3.6, the optimal spreads and market order execution boundary for a liquidating agent allowed to implement market orders is shown. The notable difference in the optimal spreads between this case and that of an agent that does not execute market orders is that presently, they are bounded below by $\max \left(0, \frac{1}{\kappa}-\frac{\Delta}{2}\right)$, while without market orders they are bounded below by 0 . This is easily seen from the feedback form of the spreads, $\delta=\left(\frac{1}{\kappa}-h_{q-1}+h_{q}\right)_{+}$, combined with the inequality $h_{q-1}-h_{q}-\frac{\Delta}{2} \leq 0$. 


\subsubsection{The effects of ambiguity aversion on market order execu- tion}

This section will investigate the effects of ambiguity on the liquidating agent's use of market orders. These effects will be observed as a modification to the market order exercise curve in the $(t, q)$ plane depending on the levels of ambiguity. Each type of ambiguity will modify the quasi-variational inequality satisfied by the value function; the infinitesimal generator is modified to use the dynamics in the candidate measure, and an entropic penalization term is added. The feedback form of the optimal controls will be the same in each case, but they will result in quantitatively different spreads due to the change in the value function. The appropriate equations are:

\section{Ambiguity on market order arrival rate}

$$
\begin{aligned}
\max \left\{\left[\partial_{t} h^{\varphi_{\lambda}}+\alpha q+\sup _{\delta \geq 0} \inf _{g}\left\{\lambda e^{g} e^{-\kappa \delta}\left(\delta+h_{q-1}^{\varphi \lambda}-h_{q}^{\varphi_{\lambda}}\right) \mathbb{1}_{q \neq 0}\right.\right.\right. \\
\left.\left.\left.+\frac{1}{\varphi_{\lambda}} \lambda\left(e^{g}(g-1)+1\right)\right\}\right],\left[h_{q-1}^{\varphi_{\lambda}}-h_{q}^{\varphi_{\lambda}}-\frac{\Delta}{2}\right]\right\}=0
\end{aligned}
$$

\section{Ambiguity on fill probability}

$$
\begin{aligned}
\max \left\{\left[\partial_{t} h^{\varphi_{\kappa}}+\alpha q+\sup _{\delta \geq 0} \inf _{g \in \mathcal{G}}\left\{\lambda \int_{\delta}^{\infty} e^{g(y)} F(y) d y\left(\delta+h_{q-1}^{\varphi_{\kappa}}-h_{q}^{\varphi_{\kappa}}\right) \mathbb{1}_{q \neq 0}\right.\right.\right. \\
\left.\left.\left.\quad+\frac{1}{\varphi_{\kappa}} \lambda \int_{0}^{\infty} e^{g(y)}(g(y)-1)+1 F(y) d y\right\}\right],\left[h_{q-1}^{\varphi_{\kappa}}-h_{q}^{\varphi_{\kappa}}-\frac{\Delta}{2}\right]\right\}=0
\end{aligned}
$$

\section{Ambiguity on midprice drift}

$$
\begin{array}{r}
\max \left\{\left[\partial_{t} h^{\varphi_{\alpha}}+\sup _{\delta \geq 0} \inf _{\eta}\left\{\lambda e^{-\kappa \delta}\left(\delta+h_{q-1}^{\varphi_{\alpha}}-h_{q}^{\varphi_{\alpha}}\right) \mathbb{1}_{q \neq 0}+\eta q+\frac{1}{2 \varphi_{\alpha}}\left(\frac{\alpha-\eta}{\sigma}\right)^{2}\right\}\right]\right. \\
\left.\left[h_{q-1}^{\varphi_{\alpha}}-h_{q}^{\varphi_{\alpha}}-\frac{\Delta}{2}\right]\right\}=0
\end{array}
$$

The effect of ambiguity on the MO execution boundary can be explained with similar reasoning to the change in the optimal spreads previously discussed. The most notable feature in Figure 3.7 is the magnitude of the change for an agent that is ambiguity averse to the midprice drift. The significant change in MO execution strategy can be intuitively understood again by interpreting this type of ambiguity as a cumulative inventory 


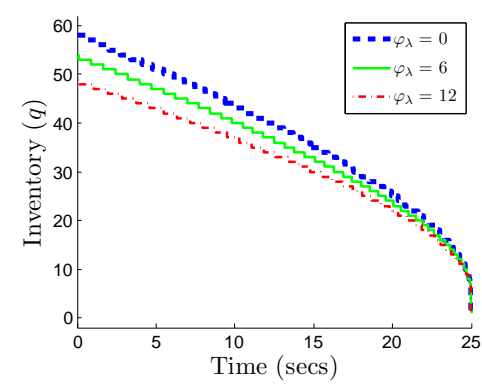

(a) MO arrival rate

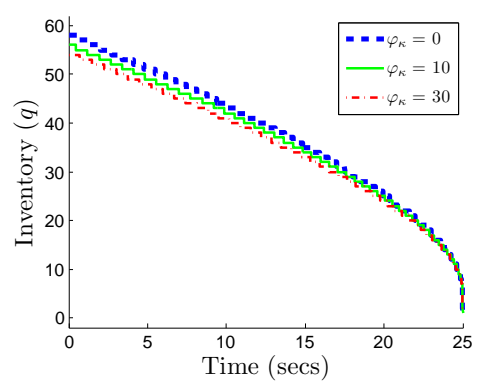

(b) Fill probability

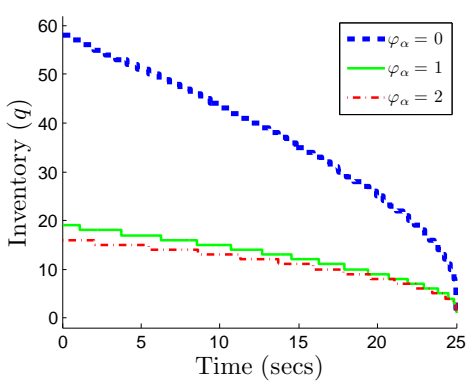

(c) Midprice drift

Figure 3.7: Effect on market order execution boundary for an agent that is ambiguity averse to different factors of the reference model. Parameter values are $\kappa=15, \lambda=2$, $\sigma=0.01, \alpha=0, \ell(q)=\theta q, \theta=0.01, Q=60$ and $T=25$.

penalty. The agent is strongly encouraged to sell shares very quickly with this type of penalty, much more so than compared to only the terminal inventory penalty.

The relatively small change due to ambiguity on fill probability can be understood as follows: for large inventory positions, the natural inclination of the agent is to post small spreads because they are in a hurry to liquidate their position before maturity. But as the agent posts smaller spreads, the change in the execution price distribution must be larger to have a significant impact on the fill probability and hence also the performance of the strategy. This type of large change is prevented by the entropic penalty. Essentially, by naturally wanting to post small spreads, the agent has already gained a significant amount of protection against a misspecified fill probability.

Ambiguity with respect to $\mathrm{MO}$ arrival rate lies in a middle ground. On one hand, the significance of this type of ambiguity is less than that of the midprice drift because changing the arrival rate does not directly penalize the holding of inventory. On the other hand, posting smaller spreads naturally for larger inventory positions does not provide a natural protection against a change in the arrival rate as it did for a change in the distribution of trade executions.

\subsubsection{Numerical implementation with market orders}

In Section 2.6, the numerical scheme used for the equations governing the market making problem was outlined. This section will outline the numerical scheme used for the liquidation problem which includes the numerical implementation of market orders. Once 
again, the equations that are of interest in this section are equations $(3.20),(3.21),(3.22)$, and (3.23), each of which can be written in the form:

$$
\begin{aligned}
\max \left\{\left(\partial_{t} h_{q}+\alpha q-\frac{1}{2} \varphi_{\alpha} \sigma^{2} q^{2}+\sup _{\delta \geq 0} f\left(h_{q-1}, h_{q}, \delta\right) \mathbb{1}_{q \neq 0}\right),\left(h_{q-1}^{\varphi_{\alpha}}-h_{q}^{\varphi_{\alpha}}-\frac{\Delta}{2}\right)\right\} & =0 \\
h_{q}(T) & =-q \ell(q)
\end{aligned}
$$

for an appropriate function $f$ which arises from substituting the optimal $g^{ \pm \lambda *}, g^{ \pm \kappa *}(y)$, and $\eta^{*}$ where applicable. This equation is treated in a fully explicit method. The time interval $[0, T]$ is subdivided into $N$ subintervals of equal length, and set $\Delta t=\frac{T}{N}$ and $t_{n}=n \Delta t$. Also set $h_{q, n}=h_{q}\left(t_{n}\right)$ and note that $h_{0, n}=0$ for all $n$. Then $h_{\cdot, n-1}$ is computed from $h_{\cdot, n}$ via the process outlined in the following pseudocode. The main addition here is that after the explicit propagation of the solution, the boundary condition of the quasi-variational inequality must be enforced:

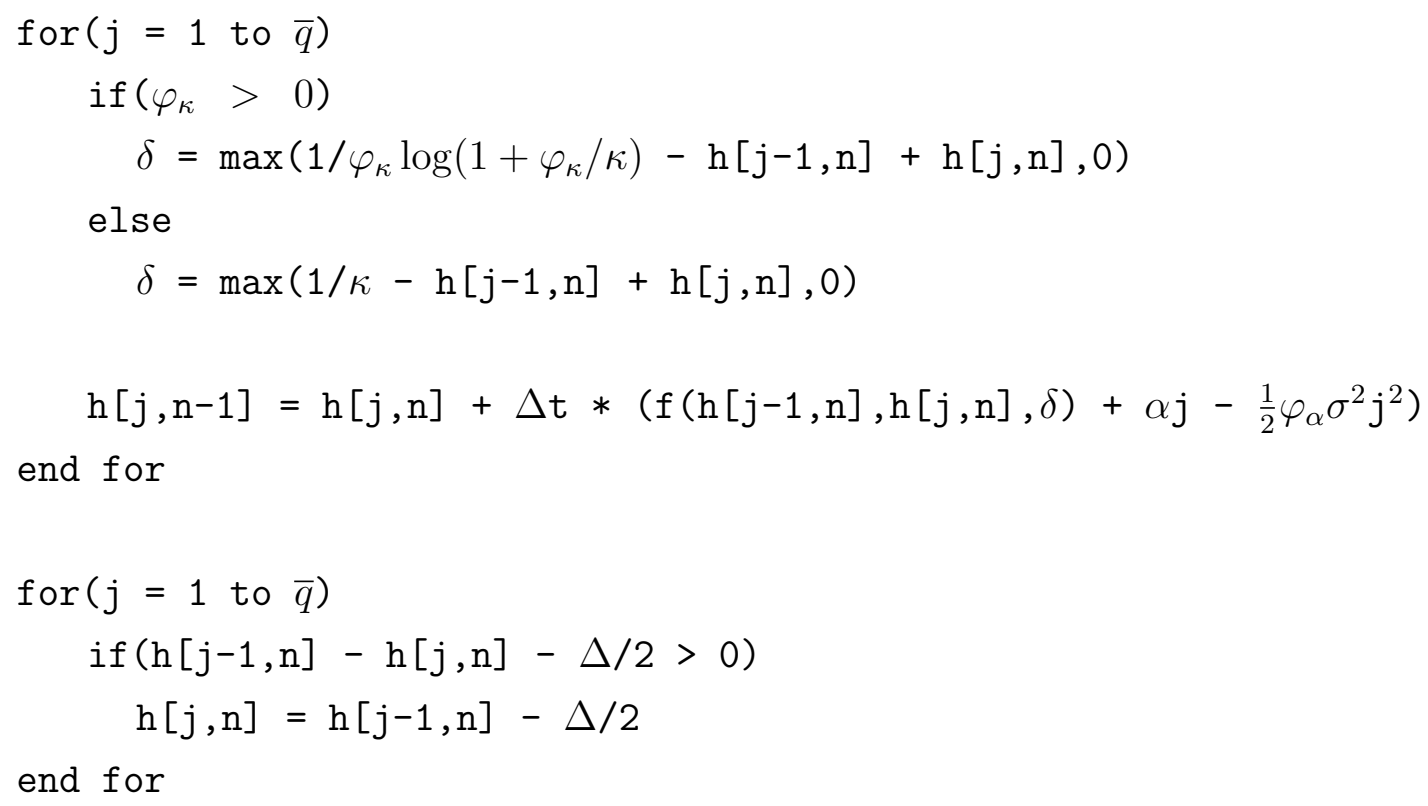




\section{Chapter 4}

\section{Volume Order Imbalance}

The main goal of this chapter will be to introduce a new reference model in the setting of the market making problem. Although the models used in Chapters 2 and 3 have been studied extensively in previous literature, albeit without ambiguity, they suffer from shortcomings when calibrated to real trade data. Specific details will be expanded upon in Section 4.1, and these results will be used to motivate some of the assumptions to be made in the new model. The most significant addition to the new model, a quantity called volume order imbalance, will be introduced in Section 4.2. This section will give motivation as to why a market maker could benefit from basing their trading strategy off of order imbalance.

\subsection{Shortcomings of Continuous Model}

This section will discuss the model introduced in Section 2.1, to which the model used in Section 3.1 is nearly identical. The two main characteristics of the model which contribute to its shortcomings are its continuous structure and non-speculative nature. By continuous structure, this makes reference to the fact that limit orders can be posted at any real valued distance $\delta \geq 0$ from the midprice. In reality, this is not the case; agents may post limit orders at integer multiples of the "tick" size (often one cent). An agent acting according to the model in Section 2.1 would have to compute their optimal trading strategy and then round it to an appropriate tick. The non-speculative nature of the model will be discussed in Section 4.2. 


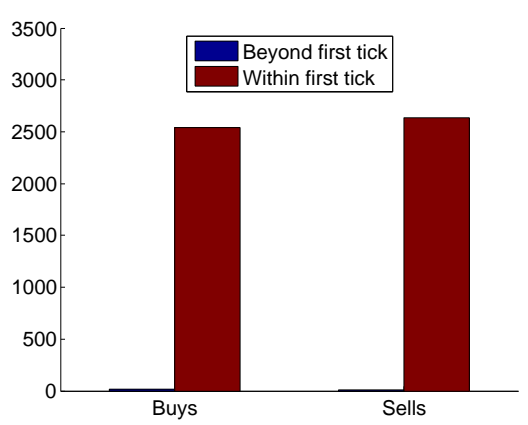

(a) BBBY

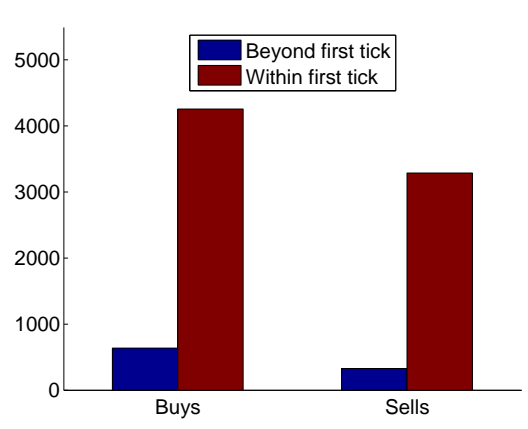

(b) MSFT

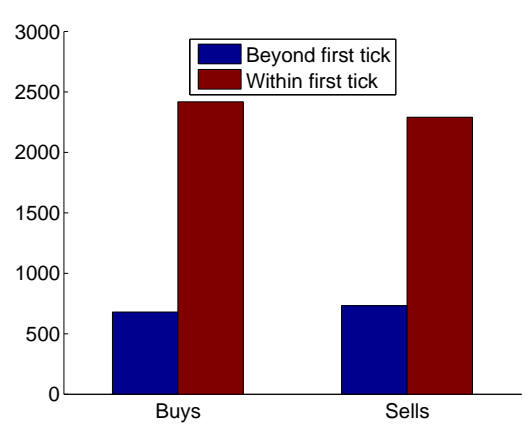

(c) TEVA

Figure 4.1: Number of market buy and sell orders that trade with limit orders at the first tick or beyond. Data is taken from a full day of trading on January 3, 2011 on the NASDAQ exchange.

\subsubsection{Tick activity}

The side effects of a continuous limit order book will be illustrated here. In Figure 4.1, data for three different equities is used to show how many market orders in a single trading day are executed at the first available tick past the midprice, versus how many are executed beyond the first tick due to a large market order volume.

Clearly, a significant proportion of all trades occur at the first tick. With a continuous range of limit order prices, the likelihood of an order to be filled by a given market order can be finely adjusted by making small changes to the limit order price. But with discrete ticks that exhibit drastically different activity throughout the day, this fine adjustment is not possible. Instead, the agent's order will either be filled with some probability at the best tick, or be filled with a probability that is an order of magnitude smaller at the next tick. The data contained in Figure 4.1 is enough to compute a crude estimate for the values of $\kappa^{ \pm}$which appear in the continuous model. These are the parameters that most directly dictate the typical posting price. This is done as follows: according to the model, if the agent posts at $\delta^{ \pm}=0$, then their probability of being filled is equal to 1 . If they increase the distance of their post by one tick, the probability that their order is filled decreases by the proportion of total trades represented by the red region in Figure 4.1. Specifically, if $n_{1}^{ \pm}$is the number of trades that execute at the first tick and $n_{2}^{ \pm}$is the number of trades that execute beyond the first tick, then:

$$
e^{-\kappa^{ \pm} \Delta}=\frac{n_{2}^{ \pm}}{n_{1}^{ \pm}+n_{2}^{ \pm}}
$$


where $\Delta$ is the tick size. Computing $\kappa^{ \pm}$via this method for the above three equities yields values:

\begin{tabular}{cccc}
\hline & BBBY & MSFT & TEVA \\
\hline$\kappa^{+}$ & 485 & 205 & 152 \\
$\frac{1}{\kappa^{+}}$ & 0.0021 & 0.0049 & 0.0066 \\
\hline$\kappa^{-}$ & 548 & 240 & 142 \\
$\frac{1}{\kappa^{-}}$ & 0.0018 & 0.0042 & 0.0070
\end{tabular}

Table 4.1: Estimation of $\kappa^{ \pm}$

Recall that the optimal posting strategy for the ambiguity neutral agent is equal to $\delta^{ \pm}=\frac{1}{\kappa^{ \pm}}-h_{q \mp 1}+h_{q}$. Although $\kappa^{ \pm}$also has effects on $h_{q}$, the main influence of $\kappa^{ \pm}$on the spread is through the first term and all of the spreads will centre around $\frac{1}{\kappa^{ \pm}}$with small variations depending on the inventory level. As seen in Table 4.1, all of the values of $\frac{1}{\kappa^{ \pm}}$lie significantly below the single tick value of 0.01 (this behaviour is consistent over different days of trade data). This will have the effect of making the optimal posting prices in the market making problem always equal to the first tick beyond the midprice. Essentially, the agent's strategy becomes static, not even changing as a function of their current inventory level. One might think that using such a strategy would result in the agent earning cash equal to the spread every time they buy and subsequently sell a share of the asset. However, this trading strategy can be shown to be extremely ineffective and risky due to the effects of adverse selection. Adverse selection is a phenomenon which results in an agent selling an asset for a price less than the original buying price and occurs due to price impact following a market order. See Figure 4.2 and Table 4.2 for the results of backtesting this strategy on a month of trade data. In the next section, a method will be demonstrated for estimating the significance of these adverse selection effects, and whether they pose a risk to the agent posting limit orders.

\begin{tabular}{cccc}
\hline & BBBY & MSFT & TEVA \\
\hline Mean & -0.347 & -1.278 & -0.706 \\
Standard Deviation & 3.236 & 9.439 & 8.030 \\
\hline
\end{tabular}

Table 4.2: Mean value and standard deviation of empirical distributions from Figure 4.2.

The disproportionate amount of activity at the fist tick also serves as motivation for the agent's class of control processes in Section 4.4 . 


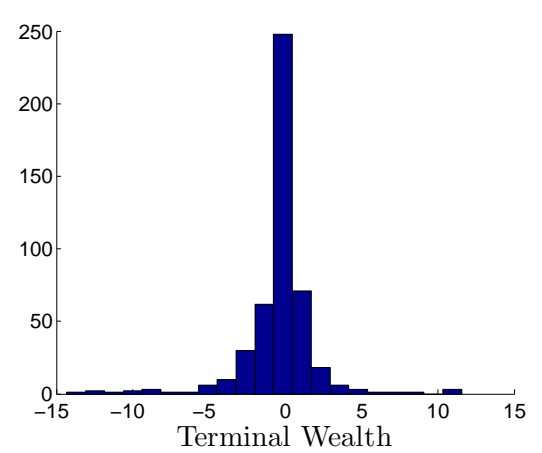

(a) BBBY

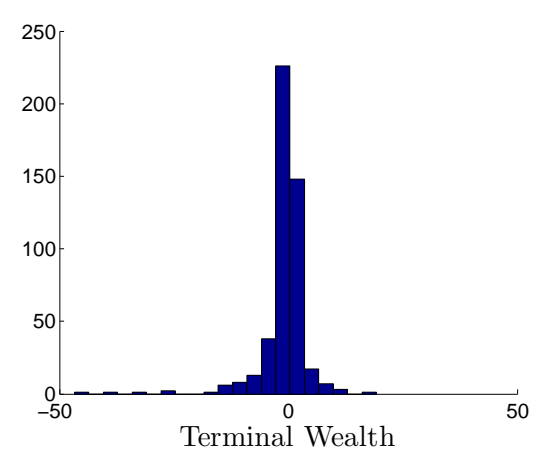

(b) MSFT

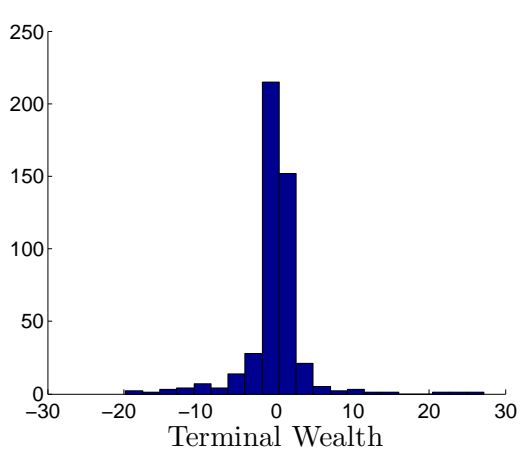

(c) TEVA

Figure 4.2: Distribution of terminal wealth achieved by always posting limit orders at the first tick. Data is taken from a full month of trading (January 2011). Each sample represents executing the strategy over a 15 minute period and liquidating terminal inventory at midprice (without any liquidation penalty).

\subsection{Imbalance as a Market Predictor}

The model of Section 2.1 is static in the sense that none of the dynamics are dependent on changing market conditions. A change in market conditions can have an effect on whether one specific trading strategy is beneficial or detrimental to the agent. Unfortunately, the agent is able to make no distinction between these cases if the model does not account for this behaviour. This section will introduce the quantity called volume order imbalance and show how it acts as an indicator of short term future market behaviour.

Volume order imbalance is defined as the proportion of limit order volume at the best bid with respect to the total volume at the best bid and ask. If the volume at the best bid (ask) is denoted $V_{t}^{b}\left(V_{t}^{a}\right)$, then imbalance may be defined:

$$
I_{t}=\frac{V_{t}^{b}}{V_{t}^{a}+V_{t}^{b}} .
$$

Clearly, $I_{t}$ is a stochastic process with $I_{t} \in[0,1]$.

\subsubsection{Market order activity}

In Figure 4.1, the total number of market buy and sell orders that touched the first tick or beyond the first tick were counted. Here, a similar counting exercise will be performed, except instead of being concerned with the ticks that are involved with the market order, 
the likelihood of a buy or sell order will be shown to depend on the value of $I_{t}$. To do so, the interval $[0,1]$ is divided into three subintervals: $[0,0.3),[0.3,0.7]$, and $(0.7,1]$ which will be referred to as the low, mid, and high regimes of imbalance respectively. The number of market buy and sell orders that occur with $I_{t}$ lying in each of these intervals is counted and displayed in Figure 4.3. The three intervals are chosen symmetrically around 0.5 , but otherwise arbitrarily, except to keep the total number of trades within each regime roughly the same over all three equities.

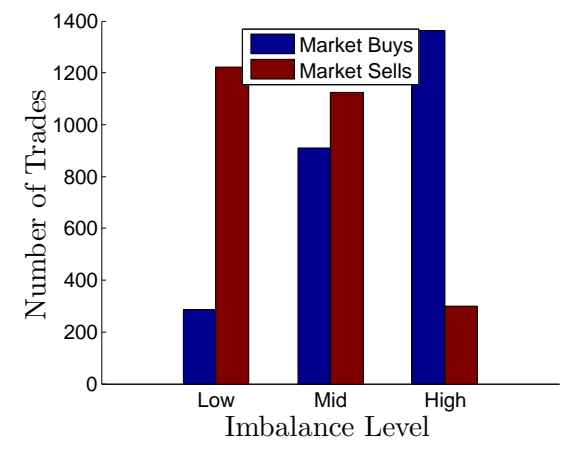

(a) BBBY

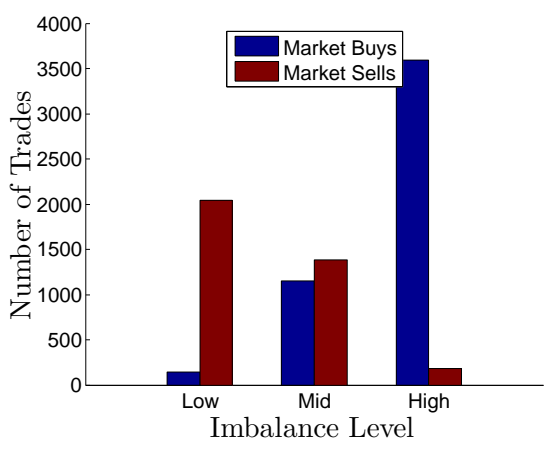

(b) MSFT

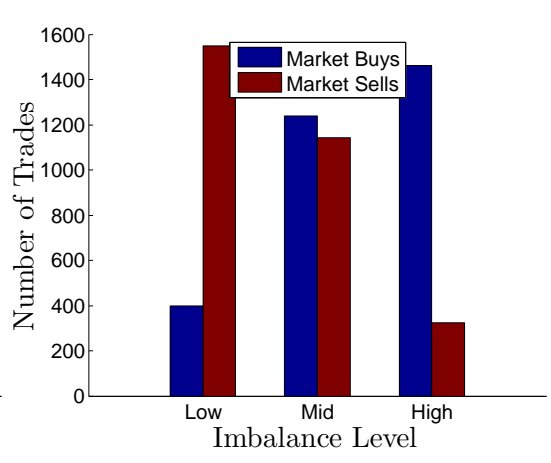

(c) TEVA

Figure 4.3: Number of market buy and sell orders that occur in each regime of imbalance. Data is taken from a full day of trading on January 3, 2011.

This is the first example of the type of behaviour that can not be captured by the model of Section 2.1 due to its static nature. If the original model were accurate, then bars of equal colour would have equal heights, independent of the imbalance regime. Clearly, acting under such a model blinds the agent to a significant source of information, namely the likelihood of the next market order being a buy or a sell. This dependence on market order intensity as a function of imbalance will be incorporated into the model in Section 4.4 .

\subsubsection{Midprice dynamics}

In addition to the regime dependent intensity of market orders, the movement of the asset's midprice will also be shown to depend on the imbalance regime. Consider once again the three intervals $[0,0.3),[0.3,0.7]$, and $(0.7,1]$. For each of these intervals, the midprice will be observed $20 \mathrm{~ms}$ after a market order, and the change with respect to the midprice immediately before the market order is computed.

In Figure 4.4, similar behaviour is observed over all three equities. Specifically, when 


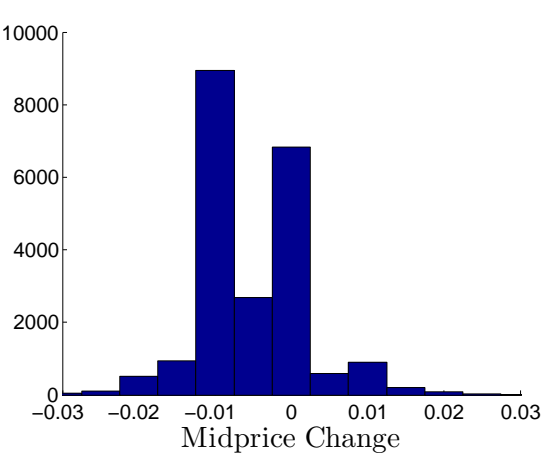

$(\text { a) })_{4}$ Low Imbalance - BBBY

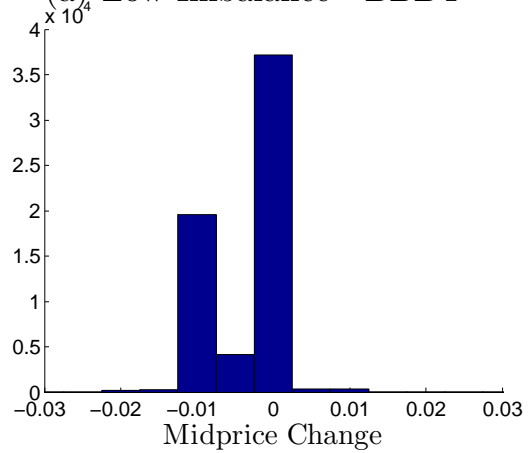

(d) Low Imbalance - MSFT

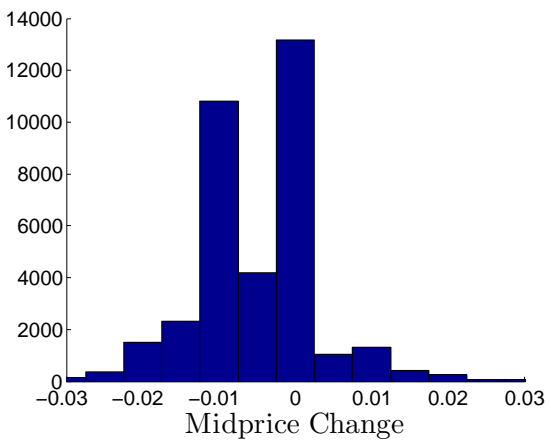

(g) Low Imbalance - TEVA

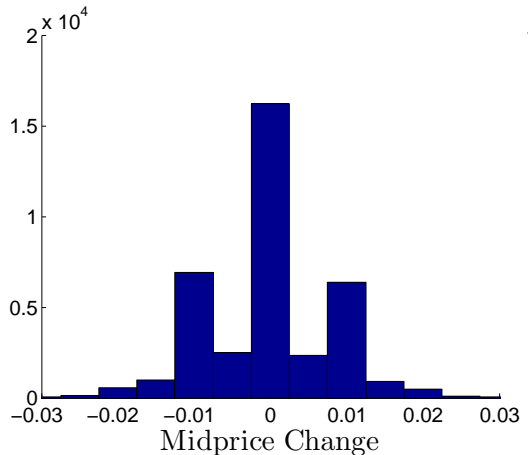

(b) Mid Imbalance - BBBY

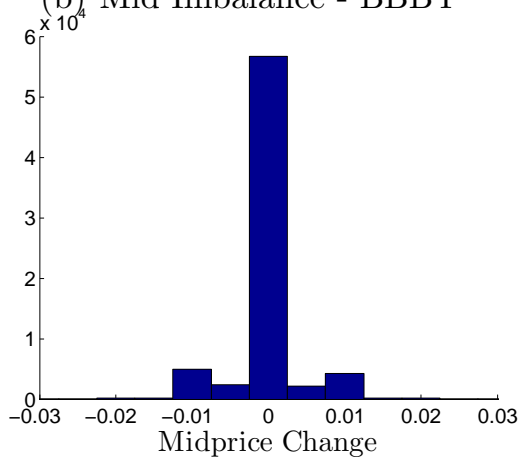

$(\mathrm{e})_{4}$ Mid Imbalance - MSFT

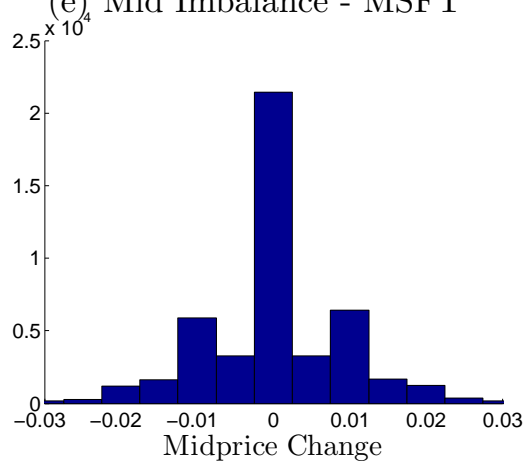

(h) Mid Imbalance - TEVA

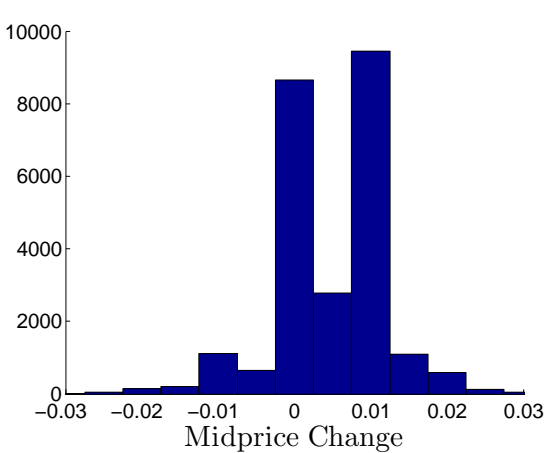

(c) ${ }_{4}$ High Imbalance - BBBY

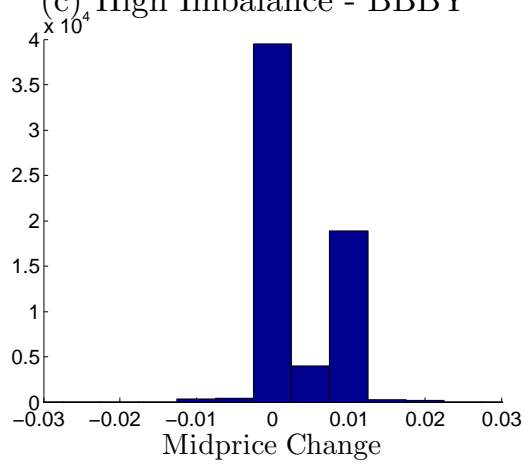

(f) High Imbalance - MSFT

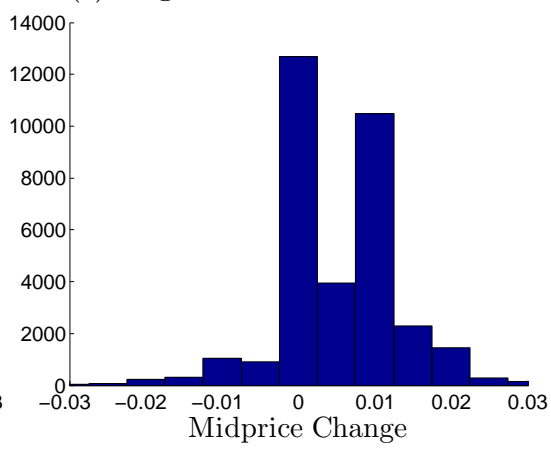

(i) High Imbalance - TEVA

Figure 4.4: Distribution of midprice changes $20 \mathrm{~ms}$ after a market order. Data is taken from a full month of trading (January 2011).

imbalance is low, the midprice generally decreases shortly after a market order. Similarly, it increases shortly after a market order when imbalance is high. The changes are roughly symmetric when imbalance is in the mid regime. This is another significant observation of market behaviour which is ignored in the model of Section 2.1.

It will also be of importance to see how these midprice changes behave not only after market orders, but after a specific type of market order (buy or sell). This decomposition is displayed in Figure 4.5 for market buy orders only. Again it is clear that there is regime dependence on the distribution of these changes. When imbalance is high, the size of 
an upward movement after a market buy order is generally larger than in the other two regimes. In the low imbalance regime, the changes are generally still positive (as would be expected after a market buy order), but the magnitude of the changes are smaller than the other two regimes. Similar behaviour occurs for market sell orders, except changes are generally negative and larger for the low imbalance regime rather than the high imbalance regime. This is the second example of behaviour that is not captured by the model of Section 2.1.

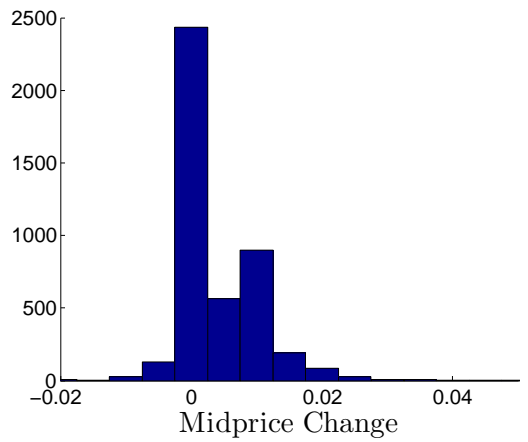

(a) Low Imbalance - BBBY

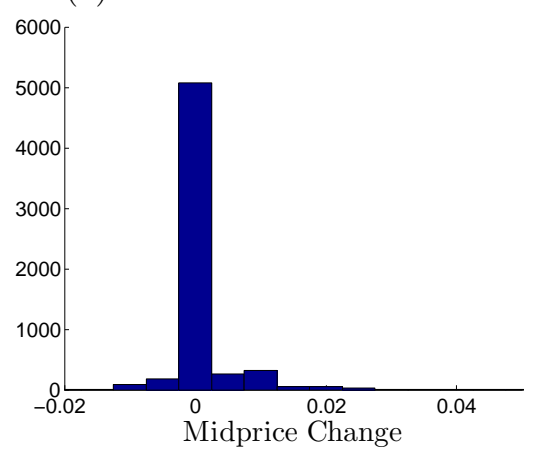

(d) Low Imbalance - MSFT

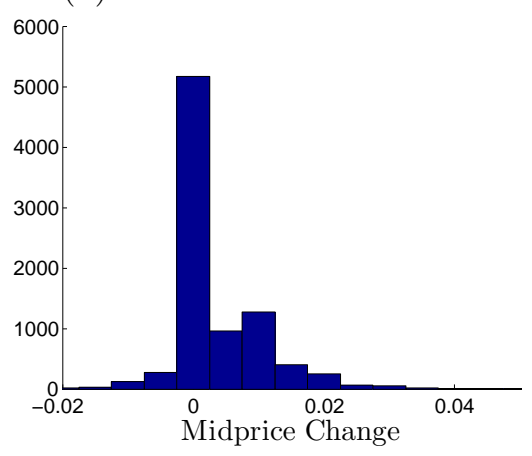

(g) Low Imbalance - TEVA

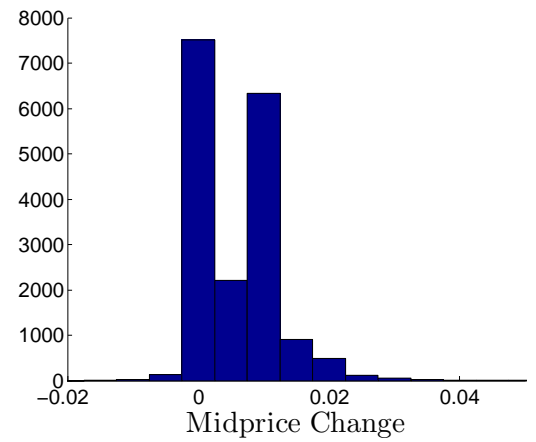

(b) Mid Imbalance - BBBY

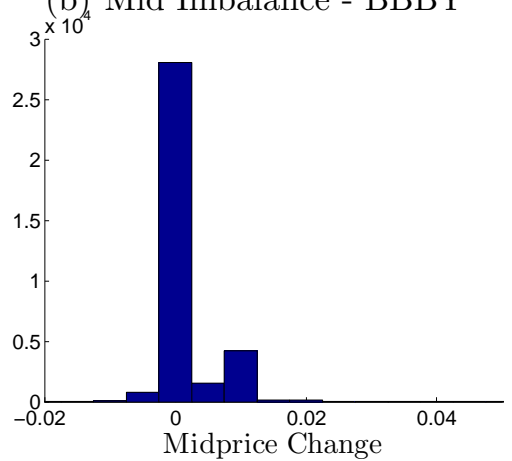

(e) Mid Imbalance - MSFT

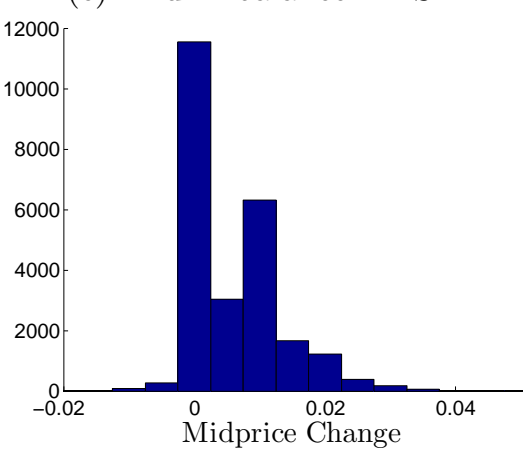

(h) Mid Imbalance - TEVA

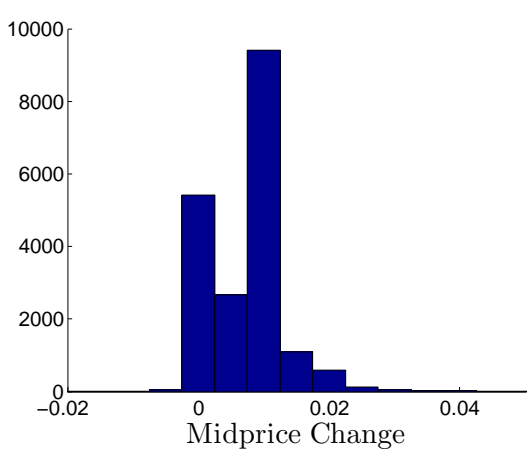

(c) High Imbalance - BBBY

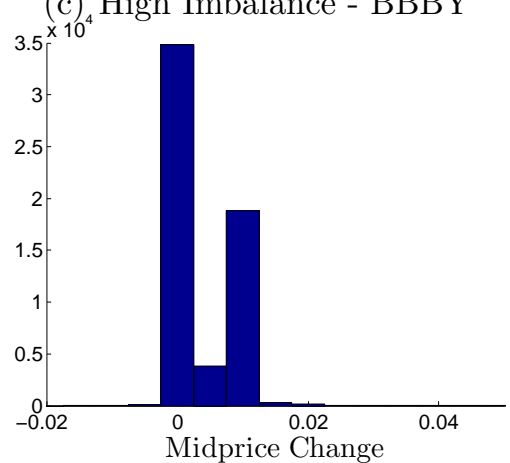

(f) High Imbalance - MSFT

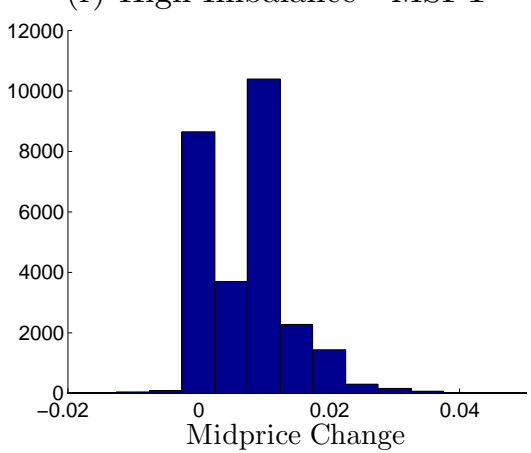

(i) High Imbalance - TEVA

Figure 4.5: Distribution of midprice changes $20 \mathrm{~ms}$ after a market buy order. Data is taken from a full month of trading (January 2011).

By ignoring the differences seen above in the short term dynamics of midprice movements and market order intensity, the agent is unable to protect themselves against adverse 
selection effects, and they are unable to take advantage of situations which would result in a benefit to their objective.

\subsection{Modelling Imbalance}

The nature of the limit order book makes the imbalance process itself a volatile pure jump process. Some of the volatility can be smoothed out by redefining the imbalance process to include limit order volumes at ticks beyond the first, or by defining it to be an average over a moving window. In Figure 4.6, the path of $I_{t}$ is shown over a time period of one minute.

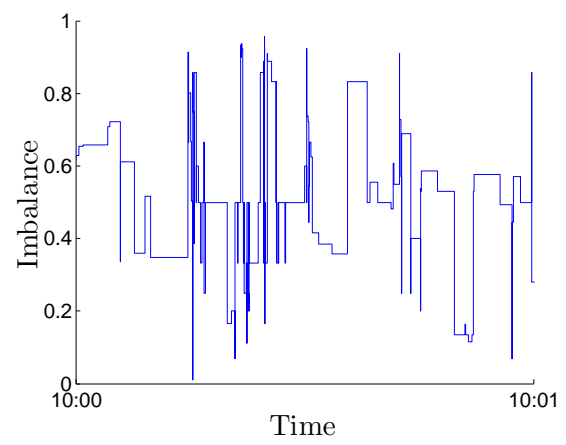

Figure 4.6: Imbalance process for TEVA on one minute of data.

At this point, a modelling decision must be made for the imbalance process. Rather than model the process of imbalance directly, the imbalance regime will be modelled using a continuous time Markov chain. This simplifying choice is motivated by the following observations:

- When imbalance is considered as a regime switching process with only three states, it has a significant amount of predictive power as seen in the previous section.

- The dependence of market order intensity and midprice changes on imbalance are very easy to calibrate using a regime switching process.

- Modelling the imbalance process directly would be significantly more complicated than modelling the regime switching process.

Up to this point, only three regime states of imbalance have been considered. This was done to simplify the demonstration of its predictive power, and also to demonstrate that 
even with a small number of regimes, its predictive power is significant. As one considers more regimes for imbalance, the resulting fine structure becomes more realistic, but the dependence of other market dynamics become more difficult to calibrate accurately. In Figure 4.7, the regime process that corresponds to the path in Figure 4.6 is shown when the number of regime states is 3 and 5 .

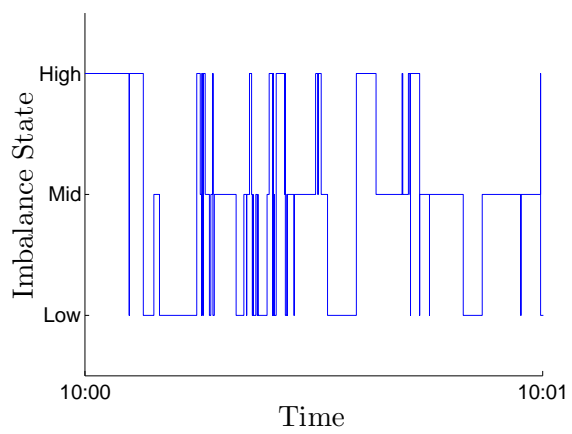

(a) Three states

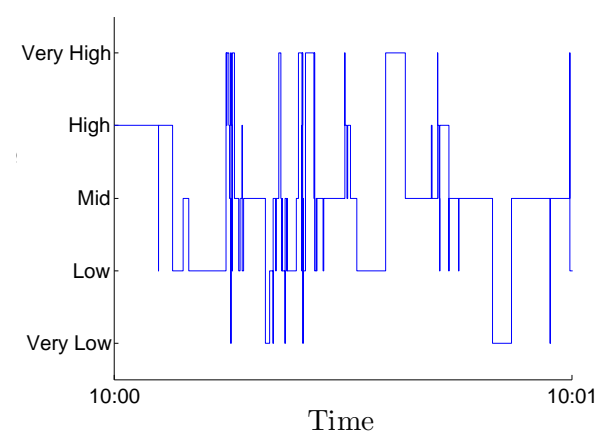

(b) Five states

Figure 4.7: Imbalance regime processes for TEVA on one minute of data.

\subsection{A New Reference Model}

In this section, dynamics for the market underliers are proposed which reflect the dependence on volume order imbalance. The factors to consider are i) imbalance itself, ii) the midprice of the asset, iii) the dynamics of market orders, and iv) the interaction between market orders and limit orders. Once these factors have specified dynamics, the agent's optimal control problem can be stated.

Order Imbalance: The imbalance process $I_{t}$ is not modelled directly. Instead, a process which acts as an approximation to $I_{t}$ is modelled as follows: divide the interval $[0,1]$ into $n$ subintervals, $U_{k}$, such that $\cup_{k=1}^{n} U_{k}=[0,1]$ and $U_{i} \cap U_{j}=\emptyset$ for all $i \neq j$. Let $Z_{t}$ be a continuous time Markov chain with generator matrix $G$ that has elements $G_{i, j}$ (recall that $\left.\mathbb{P}\left(Z_{t}=j \mid Z_{s}=i\right)=[\exp \{G(t-s)\}]_{i, j}\right)$. The process $Z_{t}$ is intended to serve as an approximation to $I_{t}$ by the relation $Z_{t}=k$ when $I_{t} \in U_{k}$. Each subinterval will be referred to as an imbalance regime, and the set of regimes will be denoted $\mathcal{Z}$.

Market Orders: Let $\mu^{ \pm}$be independent doubly stochastic Poisson random measures with compensators $\nu_{\mathbb{P}}^{ \pm}(d y, d t)=\lambda_{t}^{ \pm} F_{Z_{t}}^{ \pm}(y) d y d t$ where $\lambda_{t}^{ \pm}=\lambda^{ \pm}\left(Z_{t}\right)$. Each point in the support of $\mu^{ \pm}$represents a market buy order $(+)$or market sell order $(-)$, and specifically, 
the total number of market buy orders is $M_{t}^{+}=\int_{0}^{t} \int_{-\infty}^{\infty} \mu^{+}(d y, d t)$, and of market sell orders is $M_{t}^{-}=\int_{0}^{t} \int_{-\infty}^{\infty} \mu^{-}(d y, d t)$. With this formulation, market buy (sell) orders arrive according to a doubly stochastic Poisson process with intensity that depends directly on the imbalance regime $Z_{t}$ through the function $\lambda^{+}\left(Z_{t}\right)\left(\lambda^{-}\left(Z_{t}\right)\right)$. This dependence is intended to reflect the information conveyed in Section 4.2.1.

Midprice: The midprice of the asset is defined as:

$$
S_{t}=S_{0}+\int_{0}^{t} \int_{-\infty}^{\infty} y \mu^{+}(d y, d t)-\int_{0}^{t} \int_{-\infty}^{\infty} y \mu^{-}(d y, d t)+\xi^{+} J_{t}^{+}-\xi^{-} J_{t}^{-},
$$

where $\xi^{ \pm}$are constants and $J_{t}^{ \pm}$are Poisson processes with intensities $\beta^{ \pm}$. With this formulation, the $y$ dimension of a point in the support of $\mu^{ \pm}$represents the distance that the midprice jumps after a market buy or sell order. This jump size has probability density function $F_{Z_{t}}^{ \pm}(y)$, depending on the state at the time of the jump. The dependence of the jump distribution on $Z_{t}$ reflects the information from Section 4.2.2. The terms $\xi^{ \pm} J_{t}^{ \pm}$represent changes in the midprice that are not a result of market order executions. These can arise, for example, due to placements and cancellations of limit orders.

Consider the completed filtered probability space $\left(\Omega, \mathbb{F}, \mathcal{F}=\left\{\mathcal{F}_{t}\right\}_{0 \leq t \leq T}, \mathbb{P}\right)$ where $\mathcal{F}$ is generated by the midprice $\left(S_{t}\right)_{0 \leq t \leq T}$, the number of market buy and sell orders that have arrived $\left(M_{t}^{ \pm}\right)_{0 \leq t \leq T}$, and the imbalance regime process $\left(Z_{t}\right)_{0 \leq t \leq T}$. The MM is given control processes $\gamma_{t}^{ \pm} \in\{0,1\}$ which are $\mathcal{F}_{t}$-predictable processes and give rise to the following dynamics of the MM's inventory and wealth:

The MM is restricted to posting their buy and sell limit orders at a fixed distance from the midprice, $\frac{\Delta}{2}$, which represent only posting at the best bid and ask. The control processes $\gamma_{t}^{ \pm}$represent whether the MM posts an order or not. If $\gamma_{t}^{+}=1$, then the MM is posting a limit sell order at time $t$ at a price of $S_{t}+\frac{\Delta}{2}$. If $\gamma_{t}^{+}=0$, then the MM is not posting any limit sell order at time $t$. Similarly for the buy side. The MM is assumed to be filled by any market order that is executed as long as they have an active limit order at the time of the execution. It is not difficult to generalize to the case where the MM's limit orders are filled only by some proportion of all market orders, $\rho$. The restriction that the MM may only post at a fixed spread is motivated by the discussion in Section 4.1.1. This gives the following dynamics to the MM's inventory, $\left(q_{t}\right)_{0 \leq t \leq T}$ :

$$
d q_{t}=\gamma_{t^{-}}^{-} d M_{t}^{-}-\gamma_{t^{-}}^{+} d M_{t}^{+}
$$


If a market buy order lifts the MM's limit sell order, then their wealth increases by $S_{t^{-}}+\frac{\Delta}{2}$. Similarly, a market sell order that hits the MM's bid will decrease the MM's wealth by $S_{t^{-}}-\frac{\Delta}{2}$. If the MM does not have a limit order posted on one side of the book, then activity on that side will not change their wealth. This results in the following dynamics of wealth:

$$
d X_{t}=\gamma_{t^{-}}^{+}\left(S_{t^{-}}+\frac{\Delta}{2}\right) d M_{t}^{+}-\gamma_{t^{-}}^{-}\left(S_{t^{-}}-\frac{\Delta}{2}\right) d M_{t}^{-}
$$

The MM is risk-neutral but capital constrained, so they can not acquire large long or short inventory positions. The MM restricts their inventory such that $-\infty<\underline{q} \leq q_{t} \leq \bar{q}<\infty$. This is done by setting $\gamma_{t}^{+}=0$ whenever $q_{t}=\underline{q}$ and $\gamma_{t}^{-}=0$ whenever $q_{t}=\bar{q}$. This ensures that the inventory will never deviate beyond the finite bounds. The MM selects the strategy $\left(\gamma_{t}^{ \pm}\right)_{0 \leq t \leq T}$ which maximizes the expected terminal wealth:

$$
H(t, x, q, S, Z)=\sup _{\left(\gamma_{s}^{ \pm}\right)_{t \leq s \leq T} \in \mathcal{A}} \mathbb{E}_{t, x, q, S, Z}^{\mathbb{P}}\left[X_{T}+q_{T}\left(S_{T}-\ell\left(q_{T}\right)\right)\right]
$$

where $T$ is the terminal time of the strategy, $q_{T}$ final inventory, $\mathbb{E}_{t, x, q, S}^{\mathbb{P}}[\cdot]$ denotes $\mathbb{P}$ expectation conditional on $X_{t^{-}}=x, q_{t^{-}}=q$ and $S_{t^{-}}=S$, and $\mathcal{A}$ denotes the set of admissible strategies which are $\mathcal{F}_{t}$-predictable processes such that inventories are bounded above by $\bar{q}$ and below by $\underline{q}$. Moreover, the function $\ell$, with $\ell(0)=0$ and $\ell(q)$ increasing in $q$, is a liquidation penalty which consists of fees and market impact costs when the MM sends an MO to unwind terminal inventory. For example $\ell(q)=\theta q$ represents a linear impact when liquidating $q$ shares.

The approach to solving for the value function $H(t, x, q, S, Z)$ and the optimal posting strategies $\gamma_{t}^{ \pm}$follows along the same lines as the previous market making problem. The value function should satisfy an HJB equation of the form:

$$
\partial_{t} H+\sup _{\gamma^{ \pm}} \mathcal{L} H=0
$$

subject to terminal conditions $H(T, x, q, S, Z)=x+q(S-\ell(q))$. The form of the infinitesimal generator $\mathcal{L}$ for the relevant state variables $\left(X_{t}, q_{t}, S_{t}\right.$, and $\left.Z_{t}\right)$ will be very different from those that appeared in Chapter 2 due to the use of a completely different 
reference model. The infinitesimal generator in this scenario is given by:

$$
\begin{aligned}
& \mathcal{L} H(t, x, q, S, Z)=\beta^{+} \mathcal{D}_{L}^{+} H+\beta^{-} \mathcal{D}_{L}^{-} H \\
&+\lambda^{+}(Z) \int_{-\infty}^{\infty} \mathcal{D}_{M}^{+} H F_{Z}^{+}(y) d y \mathbb{1}_{q \neq \underline{q}}+\lambda^{-}(Z) \int_{-\infty}^{\infty} \mathcal{D}_{M}^{-} H F_{Z}^{-}(y) d y \mathbb{1}_{q \neq \bar{q}} \\
&+\sum_{k=1}^{n} G_{Z, k}(H(t, x, q, S, k)-H(t, x, q, S, Z))
\end{aligned}
$$

where the operators $\mathcal{D}_{L}^{ \pm}$act as follows:

$$
\mathcal{D}_{L}^{ \pm} H=H\left(t, x, q, S \pm \xi^{ \pm}, Z\right)-H(t, x, q, S, Z)
$$

and the operators $\mathcal{D}_{M}^{ \pm}$act as follows:

$$
\mathcal{D}_{M}^{ \pm} H=H\left(t, x \pm \gamma^{ \pm}\left(S \pm \frac{\Delta}{2}\right), q \mp \gamma^{ \pm}, S \pm y, Z\right)-H(t, x, q, S, Z)
$$

The first two terms in $\mathcal{L}$ represent the change in the value function due to changes in the midprice that are not the result of market orders. The second two terms represent the change in the value function due to market orders. These events happen with intensity $\lambda^{ \pm}(Z)$, and the effect they have on the value function depends on both the strategy of the MM at that time, $\gamma^{ \pm}$, and the magnitude of the midprice change after the market order, $y$. Since the infinitesimal generator represents the expected rate of change of the value function, an expectation must be taken over all possible jump sizes. This is what is represented by the integrals. The last term containing the summation represents the expected rate of change of the value function due to the imbalance regime switching to different states.

\subsubsection{Feedback controls}

In much the same manner as Chapters 2 and 3, the form of the infinitesimal generator and the terminal conditions allow for a convenient ansatz to be applied: $H(t, x, q, S, Z)=$ $x+q S+h_{q, Z}(t)$. Substituting this into equation (4.2) gives a system of ODE's for the 
functions $h_{q, Z}(t)$ :

$$
\begin{aligned}
& \partial_{t} h_{q, Z}+\beta^{+} \xi^{+} q-\beta^{-} \xi^{-} q+\lambda^{+}(Z) \epsilon_{Z}^{+} q-\lambda^{-}(Z) \epsilon_{Z}^{-} q \\
&+\lambda^{+}(Z) \sup _{\gamma^{+} \in\{0,1\}}\left\{\gamma^{+}\left(\frac{\Delta}{2}-\epsilon_{Z}^{+}\right)+h_{q-\gamma^{+}, Z}-h_{q, Z}\right\} \mathbb{1}_{q \neq \underline{q}} \\
&+\lambda^{-}(Z) \sup _{\gamma^{-} \in\{0,1\}}\left\{\gamma^{-}\left(\frac{\Delta}{2}-\epsilon_{Z}^{-}\right)+h_{q+\gamma^{-}, Z}-h_{q, Z}\right\} \mathbb{1}_{q \neq \bar{q}} \\
&+\sum_{k=1}^{n} G_{Z, k}\left(h_{q, k}-h_{q, Z}\right)=0, \\
& h_{q, Z}(T)=-q \ell(q),
\end{aligned}
$$

where $\epsilon_{Z}^{ \pm}=\int_{-\infty}^{\infty} y F_{Z}^{ \pm}(y) d y$ represents the expected jump size of the midprice due to a market order. Due to discrete nature of the controls, there is little hope for finding an analytical solution to this equation. The calculation of the feedback controls, however, is quite simple:

Proposition 19 (Optimal Feedback Controls). The optimal feedback controls of the HJB equation (4.3) are given by:

$$
\begin{aligned}
& \gamma^{+*}(t, q, Z)=\left\{\begin{array}{cc}
1, & \frac{\Delta}{2}-\epsilon_{Z}^{+}+h_{q-1, Z}(t)-h_{q, Z}(t)>0 \quad \text { and } q \neq \underline{q} \\
0, & \frac{\Delta}{2}-\epsilon_{Z}^{+}+h_{q-1, Z}(t)-h_{q, Z}(t) \leq 0 \quad \text { or } q=\underline{q}
\end{array}\right. \\
& \gamma^{-*}(t, q, Z)=\left\{\begin{array}{ccc}
1, & \frac{\Delta}{2}-\epsilon_{Z}^{-}+h_{q+1, Z}(t)-h_{q, Z}(t)>0 & \text { and } q \neq \bar{q} \\
0, & \frac{\Delta}{2}-\epsilon_{Z}^{-}+h_{q+1, Z}(t)-h_{q, Z}(t) \leq 0 & \text { or } q=\bar{q}
\end{array}\right.
\end{aligned}
$$

Proof. This is clear from the binary nature of the controls and form of the supremum terms in equation (4.3).

With no analytical solution available, resorting to numerical solutions allows for the optimal strategy to be shown in Figure 4.8. The parameters corresponding to this optimal strategy are the following:

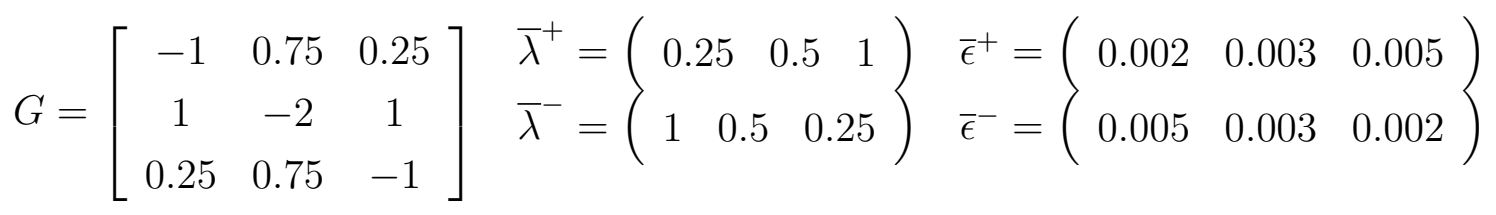

$$
\begin{aligned}
& \Delta=0.01 \quad \beta^{ \pm}=1 \quad \xi^{ \pm}=0.005
\end{aligned}
$$


This set of parameters has been chosen to reflect the impact of order imbalance on the dynamics of market orders and midprice movements. The states 1, 2, and 3 are intended to represent states of low, medium, and high imbalance respectively. The increasing (decreasing) values of $\lambda^{+}$and $\epsilon^{+}\left(\lambda^{-}\right.$and $\left.\epsilon^{-}\right)$are chosen to reflect the appropriate dependence of market order intensity and jump sizes on imbalance, as has been demonstrated in Sections 4.2.1 and 4.2.2.

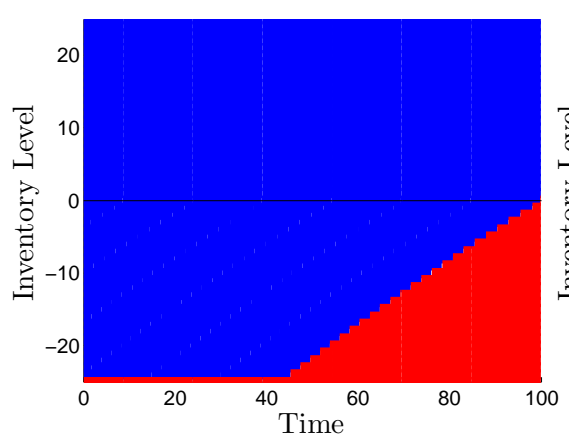

(a) Imbalance State 1 (Limit Sell)

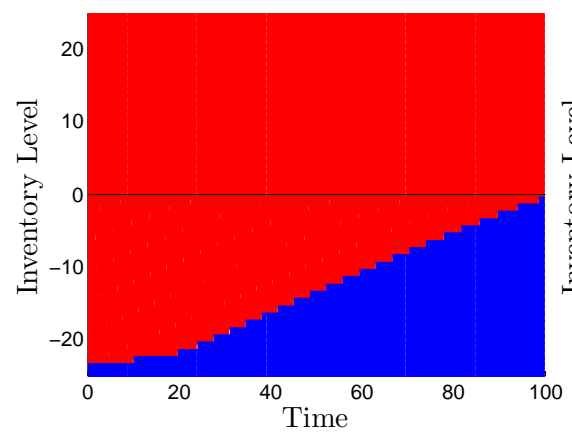

(d) Imbalance State 1 (Limit Buy)

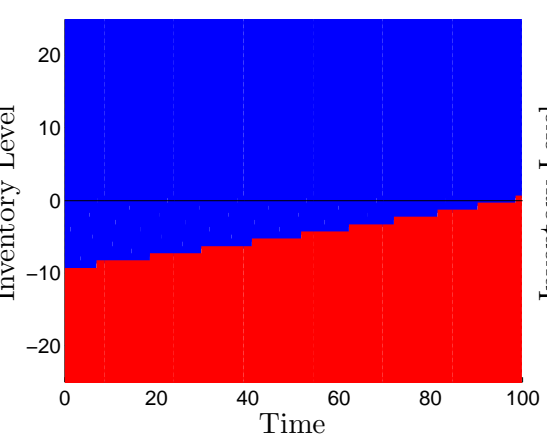

(b) Imbalance State 2 (Limit Sell)

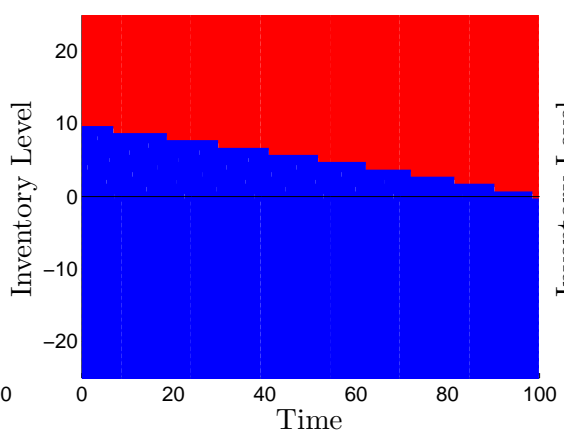

(e) Imbalance State 2 (Limit Buy)

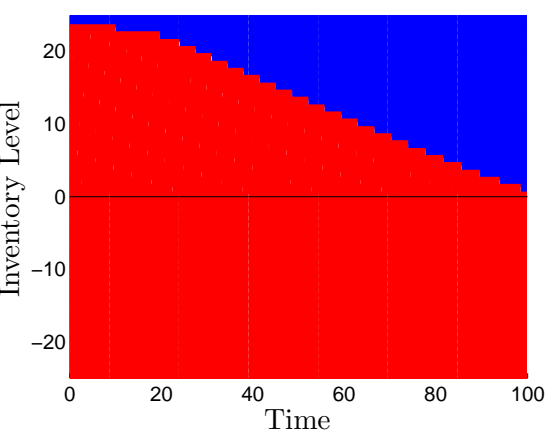

(c) Imbalance State 3 (Limit Sell)

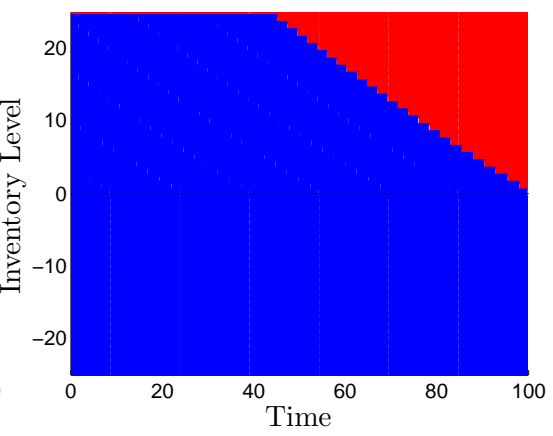

(f) Imbalance State 3 (Limit Buy)

Figure 4.8: Optimal posting strategies for parameters listed above. The agent places the corresponding limit buy or sell order posting in the blue regions and does not place a posting in the red regions. Additional parameters are $T=100, \ell(q)=-\theta q, \theta=0.005$, $\bar{q}=-\underline{q}=25$.

It is clear from the visual representation of the trading strategy in Figure 4.8 that for any single state of imbalance, there is a boundary between the trade and no-trade region. For the sake of brevity, and so that the strategy with more imbalance states can easily be plotted in the future, all of the information in Figure 4.8 is condensed into Figure 4.9 by plotting only the boundary between the two regions for each state of imbalance. 


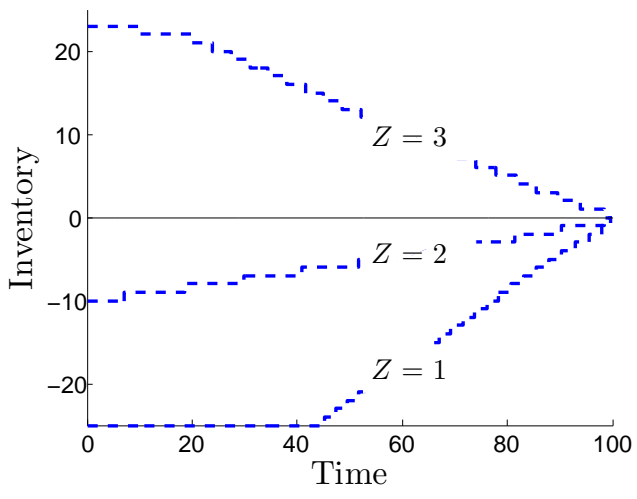

(a) Sell Boundary

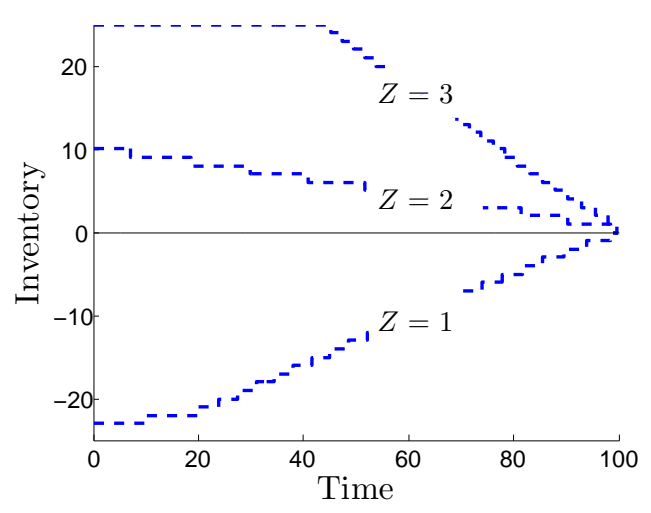

(b) Buy Boundary

Figure 4.9: The market maker posts a sell (buy) limit order if their current inventory lies above (below) the corresponding imbalance state curve.

There are two main features of the trade boundaries which can be easily explained from a financial perspective. The first is that all of the boundaries imply that at an inventory value of zero as time approaches maturity, trades are not made. This is in fact a consequence of the parameter values, namely that $\frac{\Delta}{2}-\theta-\epsilon^{ \pm}(Z)<0$ for each value of $Z$. This results in there being no benefit from executing a trade immediately before maturity when the agent is already at zero inventory, knowing that it will have to be liquidated for the same price as the initial transaction, and also suffering from a very small amount of inventory penalization. Indeed, in Figure 4.10, the trade boundaries are shown for the same set of parameters except with $\theta$ decreased to a value of 0.0005 . Note now the willingness of the MM to make trades in some circumstances immediately before maturity, because the benefit of these trades now outweighs the terminal penalty that they cost.

The second feature is the ordering of the boundaries with respect to the imbalance state. The boundary for the limit sell orders is increasing with the state label meaning that the MM is most willing to sell the asset when imbalance is low. This is a consequence of typical market behaviour when imbalance is low. Low imbalance makes market sell orders more likely, and it also makes the magnitude of a price change after a market sell order larger than after a market buy order. Together, these imply that the midprice of the asset is likely to decrease in the immediate future when imbalance is low, and so the MM should be more willing to sell shares in the low imbalance state compared to the other states. The MM will not always be willing to sell shares in the low imbalance state, however, due to both the possibility of having to pay a terminal penalty at maturity and 


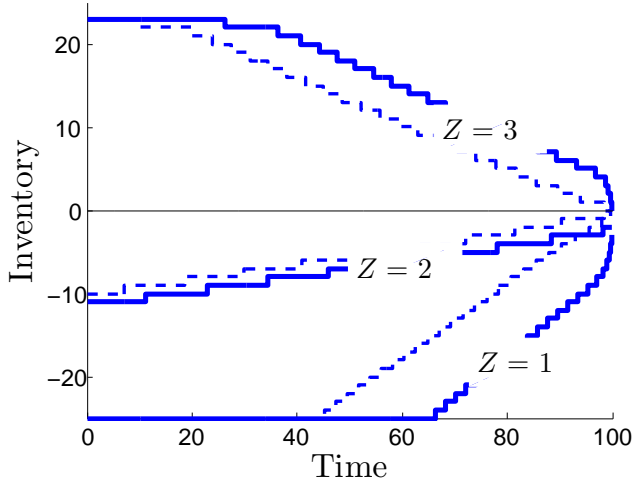

(a) Sell Boundary

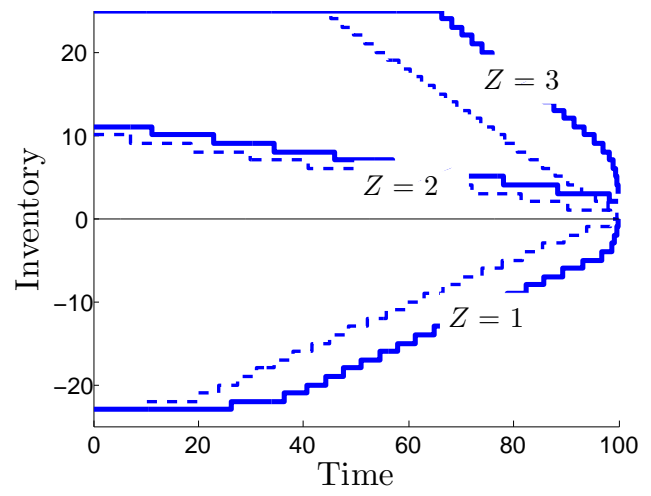

(b) Buy Boundary

Figure 4.10: The market maker posts a sell (buy) limit order if their current inventory lies above (below) the corresponding imbalance state curve. Dotted line represents original parameter set with $\theta=0.005$. Solid line uses $\theta=0.0005$.

due to the running inventory penalty.

As a final note, one additional case will be studied as a demonstration of incorporating additional imbalance regimes and observing the existence of no-trade regions. For this example, the parameter set is changed to the following:

$$
\begin{aligned}
& G=\left[\begin{array}{ccccc}
-1.75 & 0.8 & 0.5 & 0.3 & 0.15 \\
0.45 & -1.5 & 0.5 & 0.35 & 0.2 \\
0.3 & 0.7 & -2 & 0.7 & 0.3 \\
0.2 & 0.35 & 0.5 & -1.5 & 0.45 \\
0.15 & 0.3 & 0.5 & 0.8 & -1.75
\end{array}\right] \overline{\bar{\lambda}}^{-}=\begin{array}{l}
\bar{\lambda}^{+}=\left(\begin{array}{ccccc}
0.2 & 0.3 & 0.5 & 1 & 2 \\
2 & 1 & 0.5 & 0.3 & 0.2
\end{array}\right) \\
\overline{\bar{\epsilon}}^{-}=\left(\begin{array}{lllll}
0.001 & 0.002 & 0.003 & 0.006 & 0.011 \\
0.011 & 0.006 & 0.003 & 0.002 & 0.001
\end{array}\right)
\end{array} \\
& \xi^{ \pm}=0.005 \quad \beta^{ \pm}=1 \quad \Delta=0.01
\end{aligned}
$$

This results in the optimal trade boundaries shown in Figure 4.11. In Figure 4.12, both the buy and sell trade boundaries are shown for each imbalance regime. This makes it clear that there are some regions in which the market maker posts both buy and sell limit orders, and some regions in which they post no limit orders. A no-trade region is indicated by the buy boundary (solid) being below the sell boundary (dashed).

An analysis of the effects that each parameter has on the trade boundaries is not conducted directly, but rather will be performed in the context of ambiguity aversion. In the next chapter, the effects of ambiguity aversion will be considered with respect to several of the model's dynamic factors. 


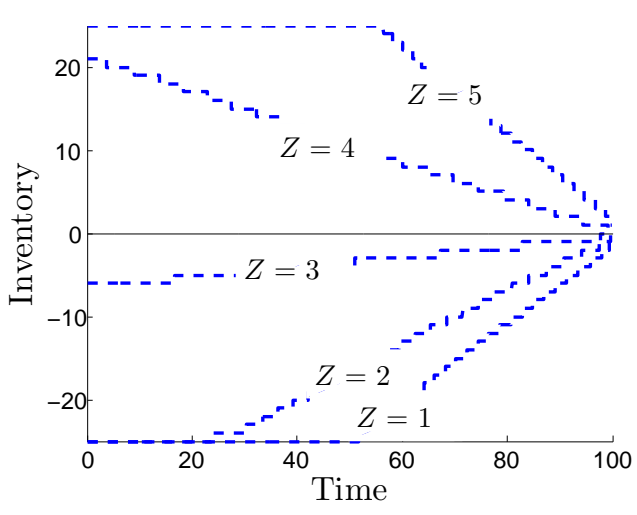

(a) Sell Boundary

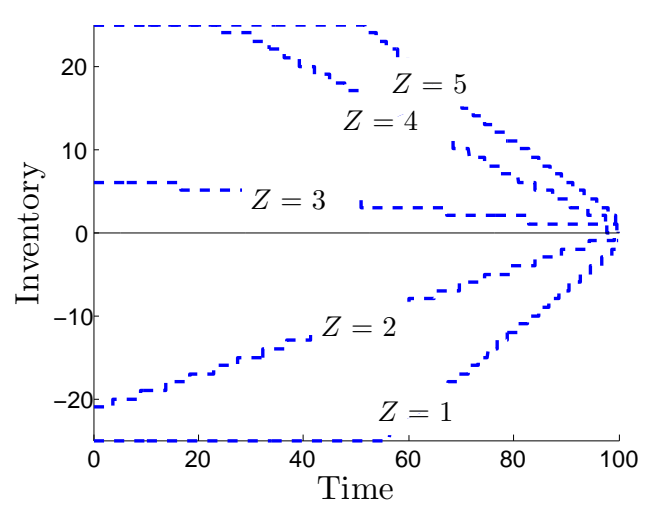

(b) Buy Boundary

Figure 4.11: The market maker posts a sell (buy) limit order if their current inventory lies above (below) the corresponding imbalance state curve. Additional parameters are $T=100, \ell(q)=-\theta q, \theta=0.005, \bar{q}=-\underline{q}=25$.

\subsection{Calibration of Imbalance Dependent Factors}

In Section 4.3, one of the reasons given for choosing a regime switching model for the imbalance process was ease of calibration. In this section, the maximum likelihood estimates for all of the imbalance dependent parameters is given based on the observation of a path of the imbalance regime process and market order executions. In addition, the values of these calibrated parameters is given when the model is restricted to be symmetric between the imbalance states.

Some notation will be introduced to make the calibration formulas more readable. Suppose a single path of the imbalance regime process, $Z_{t}$, is observed from time 0 to time $T$, along with the times of all market buy and sell orders. Let $N$ be the total number of regime switches in the observed path. For $k \in\{1, \ldots, n\}$, let $N_{k}$ be the number of disjoint time intervals in which $Z_{t}=k$, and let $T_{k}$ be the total amount of time that the process $Z_{t}$ spends equal to $k$. Let $N_{i, j}$ be the number of times that the regime process switches from state $i$ to state $j$. Finally, let $N_{i}^{ \pm}$be the number of buy and sell market orders that occurred at times when $Z_{t}=i$.

Let the generator matrix $G$ take the form $G_{i, j}=G_{i, i} p_{i, j}$ for $i \neq j$ where $G_{i, i}>0$ and $\sum_{i \neq j} p_{i, j}=1$. Then the parameters that must be calibrated are $G_{i, i}$ for $i \in\{1, \ldots, n\}$, $p_{i, j}$ for $i \in\{1, \ldots, n\}$ and $j \neq i$, and $\lambda^{ \pm}(i)$ for $i \in\{1, \ldots, n\}$. This is a total of $n^{2}+2 n$ parameters. 


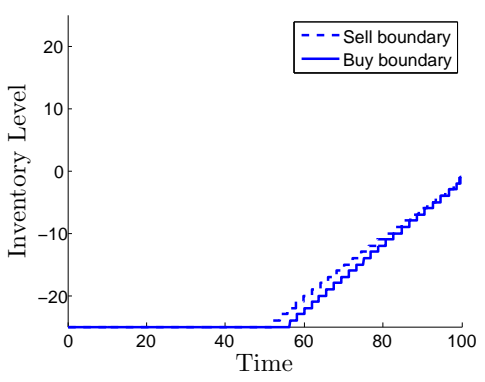

(a) Imbalance State 1

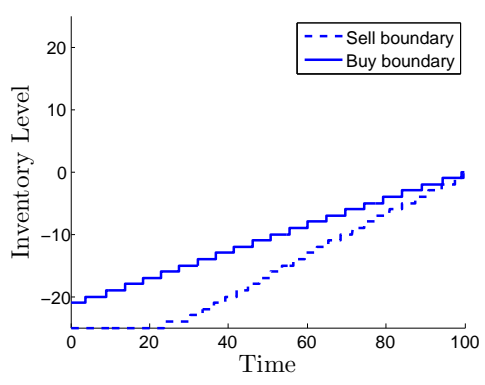

(b) Imbalance State 2

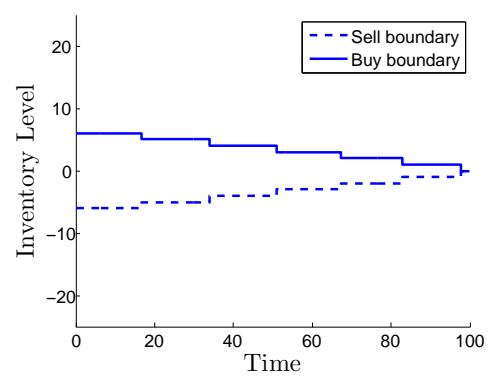

(c) Imbalance State 3

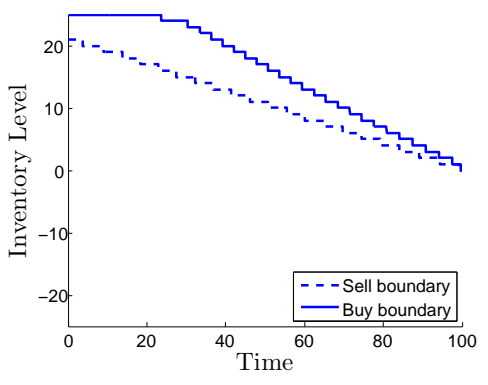

(d) Imbalance State 4

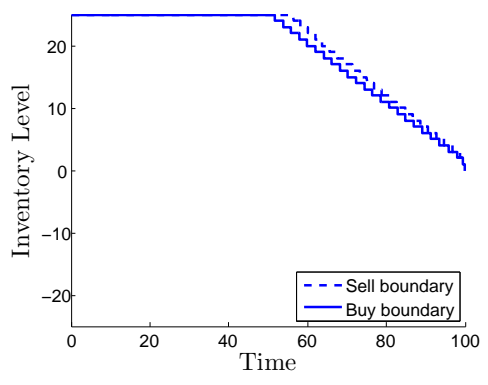

(e) Imbalance State 5

Figure 4.12: Buy (solid line) and sell (dashed line) trade boundaries for each imbalance state. No-trade regions are indicated by a dashed curve lying above a solid curve as in states 1 and 5 .

Proposition 20 (Maximum likelihood estimation of imbalance based parameters). The following formulas give the calibrated parameters of interest based on maximum likelihood estimation:

$$
G_{i, i}=\frac{N_{i}}{T_{i}} \quad p_{i, j}=\frac{N_{i, j}}{N_{i}} \quad \lambda^{ \pm}(i)=\frac{N_{i}^{ \pm}}{T_{i}}
$$

Proof. For a path of the imbalance regime process, $\left(Z_{t}\right)_{0 \leq t \leq T}$, let $\tau_{k}$ be the times at which a transition event occur with $\tau_{0}=0$ and $\tau_{M}=T$, and let $Z_{\tau_{k}}$ denote the value of $Z_{t}$ between times $\tau_{k}$ and $\tau_{k+1}$. With the notation described above, the likelihood of $\left(Z_{t}\right)_{0 \leq t \leq T}$ is:

$$
\mathcal{L}(Z)=\prod_{k=0}^{M-1} G_{Z_{\tau_{k}}, Z_{\tau_{k}}} e^{-G_{Z_{\tau_{k}}, Z_{\tau_{k}}}\left(\tau_{k+1}-\tau_{k}\right)} p_{Z_{\tau_{k}}, Z_{\tau_{k+1}}}
$$

This gives the log likelihood function:

$$
\log \mathcal{L}(Z)=\sum_{k=0}^{M-1} \log \left(G_{Z_{\tau_{k}}, Z_{\tau_{k}}}\right)-G_{Z_{\tau_{k}}, Z_{\tau_{k}}}\left(\tau_{k+1}-\tau_{k}\right)+\log \left(p_{Z_{\tau_{k}}, Z_{\tau_{k+1}}}\right)
$$


The values of $G_{i, i}$ and $p_{i, j}, i \neq j$ must be chosen to maximize this expression subject to the constraint $\sum_{j \neq i} p_{i, j}=1$ for each $i$. To enforce this constraint, Lagrange multipliers $L_{i}$ are introduced and the new objective to maximize is:

$\log \mathcal{L}(Z)=\sum_{k=0}^{M-1} \log \left(G_{Z_{\tau_{k}}, Z_{\tau_{k}}}\right)-G_{Z_{\tau_{k}}, Z_{\tau_{k}}}\left(\tau_{k+1}-\tau_{k}\right)+\log \left(p_{Z_{\tau_{k}}, Z_{\tau_{k+1}}}\right)+\sum_{i=1}^{n} L_{i}\left(\sum_{j \neq i} p_{i, j}-1\right)$

Taking a derivative of this objective with respect to $G_{i, i}$ and enforcing first order conditions gives:

$$
\begin{aligned}
\sum_{k=0}^{M-1}\left(-\mathbb{1}_{Z_{\tau_{k}}=i}\left(\tau_{k+1}-\tau_{k}\right)+\frac{\mathbb{1}_{Z_{\tau_{k}}=i}}{G_{i, i}}\right) & =0 \\
-T_{i}+\frac{N_{i}}{G_{i, i}} & =0 \\
G_{i, i} & =\frac{N_{i}}{T_{i}}
\end{aligned}
$$

This is the first desired quantity. Now taking a derivative with respect to $p_{i, j}$ and enforcing first order conditions gives:

$$
\begin{aligned}
\sum_{k=0}^{M-1} \frac{1}{p_{i, j}} \mathbb{1}_{Z_{\tau_{k}}=i, Z_{\tau_{k+1}}=j}+L_{i} & =0 \\
\frac{N_{i, j}}{p_{i, j}}+L_{i} & =0 \\
p_{i, j} & =\frac{-N_{i, j}}{L_{i}}
\end{aligned}
$$

Substituting this expression into the constraint gives:

$$
\begin{aligned}
\sum_{j \neq i} p_{i, j} & =1 \\
\sum_{j \neq i} \frac{-N_{i, j}}{L_{i}} & =1 \\
L_{i} & =\sum_{j \neq i}-N_{i, j} \\
L_{i} & =-N_{i}
\end{aligned}
$$

And so $p_{i, j}=\frac{N_{i, j}}{N_{i}}$, which is the second desired quantity. To solve for the market order 
intensities, introduce values $\tau_{k}^{ \pm}$with $\tau_{0}^{ \pm}$. For $k>0$, each $\tau_{k}^{+}$represents a time of a market buy order, and $\tau_{k}^{-}$represents a time of a market sell order. Market buy and sell orders arrive according to two independent doubly stochastic Poisson processes, and so the likelihood function for a sequence of market orders is:

$$
\mathcal{L}\left(\tau^{ \pm}\right)=\prod_{ \pm} \prod_{k=1}^{N^{ \pm}} \lambda_{\tau_{k}^{ \pm}}^{ \pm} e^{-\int_{\tau_{k-1}^{ \pm}}^{\tau_{k}^{ \pm}} \lambda_{t}^{ \pm} d t}
$$

where $\lambda_{\tau_{k}^{ \pm}}^{ \pm}$represents the intensity immediately prior to the market order at time $\tau_{k}^{ \pm}$. The corresponding log likelihood is:

$$
\log \mathcal{L}\left(\tau^{ \pm}\right)=\sum_{ \pm} \sum_{k=1}^{N^{ \pm}}\left(\log \left(\lambda_{\tau_{k}^{ \pm}}^{ \pm}\right)-\int_{\tau_{k-1}^{ \pm}}^{\tau_{k}^{ \pm}} \lambda_{t}^{ \pm} d t\right)
$$

The intensities are of the form $\lambda_{t}^{ \pm}=\lambda^{ \pm}\left(Z_{t}\right)$, so there is one value of $\lambda^{ \pm}$for each possible value of the imbalance process. To maximize the log likelihood, take a derivative with respect to $\lambda^{ \pm}(i)$ and enforce first order conditions:

$$
\begin{aligned}
\sum_{k=1}^{N^{ \pm}}\left(\frac{1}{\lambda^{ \pm}(i)} \mathbb{1}_{Z_{\tau_{k}}=i}\right)-T_{i}^{ \pm} & =0 \\
\frac{N_{i}^{ \pm}}{\lambda^{ \pm}(i)}-T_{i}^{ \pm} & =0 \\
\lambda^{ \pm}(i) & =\frac{N_{i}^{ \pm}}{T_{i}^{ \pm}}
\end{aligned}
$$

This is the third and final desired quantity.

It may be of interest for the market maker to use a model which upholds some symmetry constraints, otherwise they are essentially partaking in some form of longer term market speculation. In the following, referring to complementary states of imbalance means states of the imbalance regime process which are symmetric around the middle of $\{1, \ldots, n\}$. The complementary state of state $i$ is state $n-i+1$. If $n$ is odd, then the middle state is its own complementary state. The following desired symmetry conditions 
enforce equivalent behaviour between complementary states:

$$
\begin{aligned}
G_{i, i} & =G_{n-i+1, n-i+1} \\
p_{i, j} & =p_{n-i+1, n-j+1} \\
\lambda^{ \pm}(i) & =\lambda^{\mp}(n-i+1)
\end{aligned}
$$

Condition (4.4) comes from wanting the expected amount of time that the imbalance regime process stays in one state to be the same for its complementary state. For example, the expected amount of time that the imbalance regime is very high is the same as the expected amount of time that the imbalance regime is very low. Condition (4.5) arises from wanting the transition probabilities from one state to another to be mirrored by transition probabilities from and into the corresponding complementary states. For example, the probability of transitioning from very high imbalance to moderately high imbalance is the same as the probability of transitioning from very low imbalance to moderately low imbalance. Finally, condition (4.6) reflects having the same intensities of opposite types of market orders in complementary imbalance states. The rate of market buys in high imbalance should be the same as the rate of market sells in low imbalance, and vice versa. The correct maximum likelihood estimates with these conditions are:

Proposition 21 (Maximum likelihood estimation of symmetric imbalance based parameters). With the constraints given by equations (4.4) to (4.6), the maximum likelihood estimators are:

$$
G_{i, i}=\frac{N_{i}+N_{n-i+1}}{T_{i}+T_{n-i+1}} \quad p_{i, j}=\frac{N_{i, j}+N_{n-i+1, n-j+1}}{N_{i}+N_{n-i+1}} \quad \lambda^{ \pm}(i)=\frac{N_{i}^{ \pm}+N_{n-i+1}^{\mp}}{T_{i}+T_{n-i+1}}
$$

Proof. See Appendix B.3.

Once the formulas for these estimates are interpreted, this result makes intuitive sense. In computing $G_{i, i}$, recognize that with respect to the generator matrix, this quantity represents the rate at which the imbalance regime process transitions out of state $i$. By the nature of homogeneous continuous time Markov chains, this is the inverse of the expected amount of time that the process remains in that state for a single visit (hence, why the estimate without symmetry constraints is $G_{i, i}=\frac{N_{i}}{T_{i}}$ ). With the relationship between complementary states discussed above, it is clear that calibration information of $G_{i, i}$ comes from the time spent in state $i$, but also in state $n-i+1$. The time spent in both of these states can be used for calibration purposes for both $G_{i, i}$ and $G_{n-i+1, n-i+1}$. 
The calibration formula for $p_{i, j}$ considers not only transitions from state $i$ to state $j$ (as the non-symmetric case does), but also transitions from state $n-i+1$ to state $n-j+1$. As these states are complementary, transitions from $i$ to $j$ yield the same type of information as transitions from $n-i+1$ to $n-j+1$. Finally, the formula for $\lambda^{ \pm}(i)$ shows that information about buy orders occurring during state $i$ is complemented by information about sell orders occurring during state $n-i+1$.

An example is given below which shows the result of performing the symmetric calibration on one full day of BBBY trade data (January 3, 2011). This can be compared with a calibration which does not enforce symmetry. As expected, the symmetric parameters appear as weighted averages of the non-symmetric parameters corresponding to complementary states. The weights that appear depend on the amount of time that the imbalance process spent in each regime during this particular day.

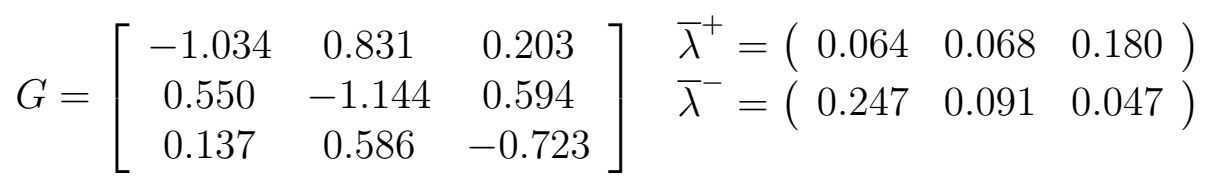

Table 4.3: Calibration of imbalance dependent factors

$$
G=\left[\begin{array}{ccc}
-0.847 & 0.683 & 0.164 \\
0.572 & -1.144 & 0.572 \\
0.164 & 0.683 & -0.847
\end{array}\right] \quad \begin{aligned}
& \bar{\lambda}^{+}=\left(\begin{array}{ccc}
0.054 & 0.080 & 0.207
\end{array}\right) \\
& \bar{\lambda}^{-}=\left(\begin{array}{lll}
0.207 & 0.080 & 0.054
\end{array}\right)
\end{aligned}
$$

Table 4.4: Calibration of imbalance dependent factors with symmetry conditions enforced 


\section{Chapter 5}

\section{Imbalance Ambiguity}

Given the imbalance modulated microstructure model developed in the last chapter, the question of how model misspecification and ambiguity aversion affect optimal strategies is able to be posed again. In the same manner as Chapters 2 and 3, the agent's level of ambiguity with respect to different factors of the model will be incorporated through the use of an entropic penalization. As the volume imbalance regime process is the most significant addition to this new model, the factors to be considered are those that have dynamics dependent on the level of imbalance: i) the arrival rate of market orders, ii) the distribution of midprice changes due to market orders, and iii) the transitions between imbalance regimes. Subsections will also be dedicated to studying the effects of ambiguity with respect to transitions with certain restrictions: i) ambiguity will only be placed on the rate of transitioning out of a state with conditional transition probabilities remaining fixed, and ii) ambiguity will only be placed on the conditional transition probabilities with the rates of transition out of a state remaining fixed. Finally, the equivalence seen in previous chapters between inventory penalization and ambiguity on midprice drift call for an analogous result now that the midprice dynamics have been fundamentally altered. The last section will compare the appropriate result for a jump process with the naive quadratic penalty.

The approach to incorporating ambiguity aversion will mimic the method of Chapters 2 and 3. A class of equivalent measures, $\mathcal{Q}$, is introduced which corresponds to a change in the dynamics of the appropriate model factor. These measure changes have an associated entropic penalty function which is added to the market maker's objective function to give a modified performance criterion. The market maker's goal is to maximize their 
performance criterion, and this is done using the standard tools of optimal stochastic control.

The modified value function is:

$$
H(t, x, q, S, Z ; \varphi)=\sup _{\left(\gamma_{u}^{ \pm}\right)_{t \leq u \leq T} \in \mathcal{A}} \inf _{\mathbb{Q} \in \mathcal{Q}} \mathbb{E}_{t, x, q, S, Z}^{\mathbb{Q}}\left[X_{T}+q_{T}\left(S_{T}-\ell\left(q_{T}\right)\right)+\mathcal{H}_{t, T}[\mathbb{Q} \mid \mathbb{P}]\right]
$$

where

$$
\mathcal{H}_{t, T}[\mathbb{Q} \mid \mathbb{P}]=\frac{1}{\varphi} \log \left\{\left(\frac{d \mathbb{Q}}{d \mathbb{P}}\right)_{T} /\left(\frac{d \mathbb{Q}}{d \mathbb{P}}\right)_{t}\right\}
$$

This value function satisfies an HJB equation of the form:

$$
\partial_{t} H+\sup _{\gamma^{ \pm}} \inf _{\mathbb{Q} \in \mathcal{Q}}\left\{\mathcal{L}^{\mathbb{Q}} H-\partial_{t} \mathbb{E}_{t, x, q, S, Z}^{\mathbb{Q}}\left[\mathcal{H}_{t, T}[\mathbb{Q} \mid \mathbb{P}]\right]\right\}=0
$$

The form of the infinitesimal generator $\mathcal{L}^{\mathbb{Q}}$ will depend on the class of measures $\mathcal{Q}$ that is being considered. As in Chapter 4, there will be little hope of finding a closed form analytical solution to equation (5.2), so numerical computations will be relied upon to study the effects on the optimal trading strategy.

\subsection{Ambiguity aversion with respect to market order arrivals}

The first type of ambiguity to be considered is on the rate of market order arrivals. The details of the class of measures used in this section are similar to those of Sections 2.2.1 and 3.2.1. Recall that in the new reference model, the number of market orders that have been executed, $M_{t}$, and the midprice, $S_{t}$, are equal to:

$$
\begin{aligned}
M_{t}^{ \pm} & =\int_{0}^{t} \int_{-\infty}^{\infty} \mu^{ \pm}(d y, d t) \\
S_{t} & =S_{0}+\int_{0}^{t} \int_{-\infty}^{\infty} y \mu^{+}(d y, d t)-\int_{0}^{t} \int_{-\infty}^{\infty} y \mu^{-}(d y, d t)+\xi^{+} J_{t}^{+}-\xi^{-} J_{t}^{-},
\end{aligned}
$$


where $\mu^{ \pm}(d y, d t)$ have compensators $\nu_{\mathbb{P}}^{ \pm}(d y, d t)=\lambda_{t}^{ \pm} F_{Z}^{ \pm}(y) d y d t$ with $\lambda_{t}^{ \pm}=\lambda^{ \pm}\left(Z_{t}\right)$. The class of equivalent measures are introduced through Radon-Nikodym derivatives of the form:

$$
\left(\frac{d \mathbb{Q}(g)}{d \mathbb{P}}\right)=\prod_{i=+,-} \exp \left\{-\int_{0}^{T} \int_{-\infty}^{\infty}\left(e^{g_{t}^{i}}-1\right) \nu_{\mathbb{P}}^{i}(d y, d t)+\int_{0}^{T} \int_{-\infty}^{\infty} g_{t}^{i} \mu^{i}(d y, d t)\right\},
$$

In the measure $\mathbb{Q}(g)$, the compensators of $\mu^{ \pm}(d y, d t)$ are $\nu_{\mathbb{Q}}(d y, d t)=e^{g_{t}^{ \pm}} \nu_{\mathbb{P}}^{ \pm}(d y, d t)$. The lack of $y$ dependence of $g_{t}^{ \pm}$is what preserves the distribution of midprice jumps and only has an effect on the rate of market order executions. The class of processes $g_{t}^{ \pm}$which are admissible is such that:

$$
\mathcal{Q}^{\lambda}=\left\{\mathbb{Q}(g): g^{ \pm} \text {are } \mathcal{F}_{t} \text {-predictable, Markov, and bounded }\right\}
$$

The entropic penalty for this class of measures satisfies:

$$
\begin{aligned}
\mathbb{E}_{t, x, q, S}^{\mathbb{Q}(g)}\left[\mathcal{H}_{t, T}^{\varphi_{\lambda}}[\mathbb{Q}(g) \mid \mathbb{P}]\right] & =\frac{1}{\varphi_{\lambda}} \mathbb{E}_{t, x, q, S}^{\mathbb{Q}(g)}\left[\sum_{i=+,-} \int_{t}^{T} \int_{-\infty}^{\infty}\left(e^{g_{u}^{i}}\left(g_{u}^{i}-1\right)+1\right) \nu_{\mathbb{P}}^{i}(d y, d u)\right] \\
& =\frac{1}{\varphi_{\lambda}} \mathbb{E}_{t, x, q, S}^{\mathbb{Q}(g)}\left[\sum_{i=+,-} \lambda^{i} \int_{t}^{T}\left(e^{g_{u}^{i}}\left(g_{u}^{i}-1\right)+1\right) d u\right],
\end{aligned}
$$

and the infinitesimal generator $\mathcal{L}^{\mathbb{Q}(g)}$ is given by:

$$
\begin{aligned}
& \mathcal{L}^{\mathbb{Q}(g)} H^{\lambda}\left(t, x, q, S, Z ; \varphi_{\lambda}\right)=\beta^{+} \mathcal{D}_{L}^{+} H^{\lambda}+\beta^{-} \mathcal{D}_{L}^{-} H^{\lambda} \\
&+e^{g_{t}^{+}} \lambda^{+}(Z) \int_{-\infty}^{\infty} \mathcal{D}_{M}^{+} H^{\lambda} F_{Z}^{+}(y) d y \mathbb{1}_{q \neq \underline{q}}+e^{g_{t}^{-}} \lambda^{-}(Z) \int_{-\infty}^{\infty} \mathcal{D}_{M}^{-} H^{\lambda} F_{Z}^{-}(y) d y \mathbb{1}_{q \neq \bar{q}} \\
& \quad+\sum_{k=1}^{n} G_{Z, k}\left(H^{\lambda}\left(t, x, q, S, k ; \varphi_{\lambda}\right)-H^{\lambda}\left(t, x, q, S, Z ; \varphi_{\lambda}\right)\right) .
\end{aligned}
$$


The same ansatz can be made as in the case with no ambiguity, $H^{\lambda}\left(t, x, q, S, Z ; \varphi_{\lambda}\right)=$ $x+q S+h_{q, Z}^{\varphi_{\lambda}}(t)$, and the resulting equations for $h_{q, Z}^{\varphi_{\lambda}}(t)$ are:

$$
\begin{aligned}
\partial_{t} h_{q, Z}^{\varphi_{\lambda}}+\beta^{+} \xi^{+} q-\beta^{-} \xi^{-} q & \\
+\sum_{ \pm} \sup _{\gamma^{ \pm} \in\{0,1\}} \inf _{g^{ \pm}}\left\{e^{g^{ \pm}} \lambda^{ \pm}(Z)\right. & \left(\gamma^{ \pm} \frac{\Delta}{2}-\left(\gamma^{ \pm} \mp q\right) \epsilon_{Z}^{ \pm}+h_{q \mp \gamma^{ \pm}, Z}^{\varphi_{\lambda}}-h_{q, Z}^{\varphi_{\lambda}}\right) \\
\left.+\frac{\lambda^{ \pm}(Z)}{\varphi_{\lambda}}\left(e^{g^{ \pm}}\left(g^{ \pm}-1\right)+1\right)\right\}+\sum_{k=1}^{n} G_{Z, k}\left(h_{q, k}^{\varphi_{\lambda}}-h_{q, Z}^{\varphi_{\lambda}}\right) & =0 \\
h_{q, Z}^{\varphi_{\lambda}}(T) & =-q \ell(q),
\end{aligned}
$$

Proposition 22 (Optimal Feedback Controls). The optimal feedback controls of equation (5.4) are given by:

$$
\begin{aligned}
& \gamma^{+*}\left(t, q, Z ; \varphi_{\lambda}\right)=\left\{\begin{array}{lcc}
1, & \frac{\Delta}{2}-\epsilon_{Z}^{+}+h_{q-1, Z}^{\varphi_{\lambda}}(t)-h_{q \lambda}^{\varphi_{\lambda}}(t)>0 & \text { and } q \neq \underline{q} \\
0, & \frac{\Delta}{2}-\epsilon_{Z}^{+}+h_{q-1, Z}^{\varphi_{\lambda}}(t)-h_{q, Z}^{\varphi_{\lambda}}(t) \leq 0 & \text { or } q=\underline{q}
\end{array},\right. \\
& \gamma^{-*}\left(t, q, Z ; \varphi_{\lambda}\right)=\left\{\begin{array}{ccc}
1, & \frac{\Delta}{2}-\epsilon_{Z}^{-}+h_{q+1, Z}^{\varphi_{\lambda}}(t)-h_{q, Z}^{\varphi_{\lambda}}(t)>0 & \text { and } q \neq \bar{q} \\
0, & \frac{\Delta}{2}-\epsilon_{Z}^{-}+h_{q+1, Z}^{\varphi_{\lambda}}(t)-h_{q, Z}^{\varphi_{\lambda}}(t) \leq 0 & \text { or } q=\bar{q}
\end{array},\right. \\
& g^{ \pm *}\left(t, q, Z ; \varphi_{\lambda}\right)=-\varphi_{\lambda}\left(\gamma^{ \pm} \frac{\Delta}{2}-\left(\gamma^{ \pm} \mp q\right) \epsilon_{Z}^{ \pm}+h_{q \mp \gamma^{ \pm}, Z}^{\varphi_{\lambda}}(t)-h_{q, Z}^{\varphi_{\lambda}}(t)\right) .
\end{aligned}
$$

Proof. See Appendix B.4.

\section{Discussion and financial interpretation}

Substitution of $g^{ \pm *}\left(t, q, Z ; \varphi_{\lambda}\right)$ into equation (5.4) gives an equation which is easily solved numerically:

$$
\begin{aligned}
& \partial_{t} h_{q, Z}^{\varphi_{\lambda}}+\beta^{+} \xi^{+} q-\beta^{-} \xi^{-} q \\
&+\sum_{ \pm} \sup _{\gamma^{ \pm} \in\{0,1\}}\left\{\frac{\lambda^{ \pm}(Z)}{\varphi_{\lambda}}\left(1-e^{-\varphi_{\lambda}\left(\gamma^{ \pm} \frac{\Delta}{2}-\left(\gamma^{ \pm} \mp q\right) \epsilon_{Z}^{ \pm}+h_{q \mp \gamma^{ \pm}, Z^{\varphi}}^{\varphi_{\lambda}}-h_{q, Z}^{\varphi_{\lambda}}\right)}\right)\right\} \\
&+\sum_{k=1}^{n} G_{Z, k}\left(h_{q, k}^{\varphi_{\lambda}}-h_{q, Z}^{\varphi_{\lambda}}\right)=0 \\
& h_{q, Z}^{\varphi_{\lambda}}(T)=-q \ell(q)
\end{aligned}
$$

Numerically solving for the value function allows for the trade boundaries to be computed. These are shown in Figure 5.1 for the following reference model parameters (these are 
the same set of five-state parameters used in Section 19):

$$
\begin{aligned}
& G=\left[\begin{array}{ccccc}
-1.75 & 0.8 & 0.5 & 0.3 & 0.15 \\
0.45 & -1.5 & 0.5 & 0.35 & 0.2 \\
0.3 & 0.7 & -2 & 0.7 & 0.3 \\
0.2 & 0.35 & 0.5 & -1.5 & 0.45 \\
0.15 & 0.3 & 0.5 & 0.8 & -1.75
\end{array}\right] \begin{array}{l}
\bar{\lambda}^{+}=\left(\begin{array}{ccccc}
0.2 & 0.3 & 0.5 & 1 & 2
\end{array}\right) \\
\bar{\lambda}^{-}=\left(\begin{array}{ccccc}
2 & 0.5 & 0.3 & 0.2
\end{array}\right) \\
\bar{\epsilon}^{-}=\left(\begin{array}{lllll}
0.001 & 0.002 & 0.003 & 0.006 & 0.011 \\
0.011 & 0.006 & 0.003 & 0.002 & 0.001
\end{array}\right)
\end{array} \\
& \xi^{ \pm}=0.005 \quad \beta^{ \pm}=1 \quad \Delta=0.01
\end{aligned}
$$

Observing the change in trade boundaries in Figure 5.1, the effect of ambiguity aversion

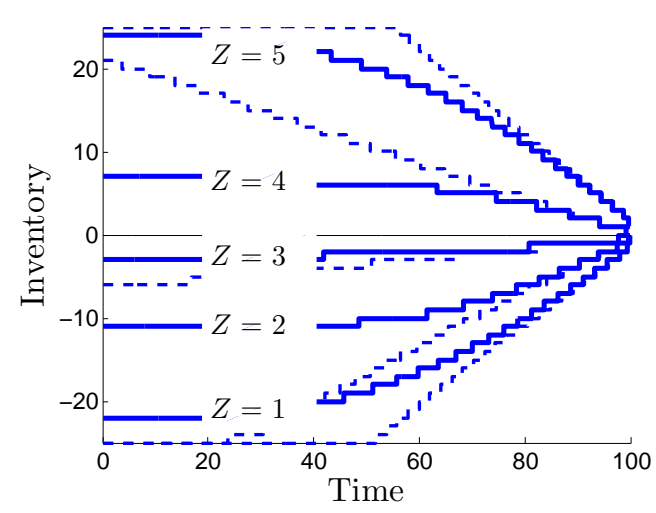

(a) Sell Boundary

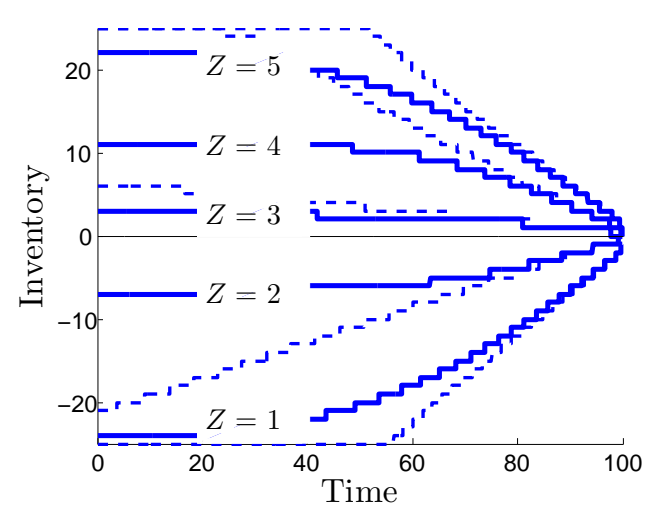

(b) Buy Boundary

Figure 5.1: Trade boundaries for a market maker that is ambiguity averse to market order arrival rate (dashed lines are ambiguity neutral boundaries). The market maker places a limit sell (buy) order if their inventory lies above (below) the corresponding state curve. Additional parameters are $\varphi_{\lambda}=0.2, T=100, \ell(q)=-\theta q, \theta=0.005, \bar{q}=-\underline{q}=25$.

on market order arrival is to generally hold less inventory. Consider the change in the sell boundaries: each ambiguity neutral curve that lies above zero (which correspond to the high and very high imbalance states) has been moved downward. In the ambiguity neutral case, when the market maker holds positive inventory and the imbalance state indicates that short term price movements will likely be positive, then they wish to remain in a positive inventory state. When the market maker becomes ambiguity averse, they are willing to do more selling, moving them further out of this positive state, because they are less certain that prices will in fact move upwards. Conversely, each ambiguity neutral curve that lies below zero has been moved upwards. When the imbalance state indicates that short term price changes will be negative, the ambiguity neutral agent would like to sell shares to a certain negative threshold. When the agent is ambiguity averse, these 
thresholds remain negative but are closer to zero. This is indicative of the agent's fear that these downward price movements may not occur as fast as their model specifies, and so holding negative inventory is not as beneficial as to the ambiguity neutral agent.

\subsection{Ambiguity aversion with respect to jump distri- bution}

In this section, the focus will be on the distribution of midprice jumps due to the arrival of a market order, $F_{Z}^{ \pm}(y)$. The following additional technical assumption is put on the allowed form of distributions: for some neighbourhood $U$ of 0 ,

$$
\int_{-\infty}^{\infty} y e^{a y} F_{Z}^{ \pm}(y) d y<\infty
$$

for all $a \in U$. The analysis in this section will encounter issues of existence of certain integrals, which correspond to whether the infimum in equations (5.1) and (5.2) is actually attained by a particular measure $\mathbb{Q}$. The condition in equation (5.6) will be used for two purposes: first, it will establish that there exists sufficiently small $\varphi_{\epsilon}$ such that all associated integrals are finite and the analysis proceeds as usual. Second, in the case where the agent does not have a sufficiently small level of ambiguity, the condition will be used in the proof that derives the form of the optimal feedback controls to show that in fact there are cases where the infimum is equal to $-\infty$ but not attained by an admissible measure.

Equivalent measures are defined through the Radon-Nikodym derivatives:

$$
\left(\frac{d \mathbb{Q}(g)}{d \mathbb{P}}\right)=\prod_{i=+,-} \exp \left\{-\int_{0}^{T} \int_{-\infty}^{\infty}\left(e^{g_{t}^{i}(y)}-1\right) \nu_{\mathbb{P}}^{i}(d y, d t)+\int_{0}^{T} \int_{-\infty}^{\infty} g_{t}^{i}(y) \mu^{i}(d y, d t)\right\}
$$

and the full class of candidate measures is:

$$
\begin{aligned}
\mathcal{Q}^{\epsilon}= & \left\{\mathbb{Q}(g): g_{t}^{ \pm}(y) \text { are } \mathcal{F}_{t}\right. \text {-predictable, Markov, and satisfy } \\
& \left.\int_{-\infty}^{\infty} e^{g_{t}^{ \pm}(y)} F_{Z_{t}}^{ \pm}(y) d y=1 \text { and } \int_{-\infty}^{\infty} y e^{g_{t}^{ \pm}(y)} F_{Z_{t}}^{ \pm}(y) d y<\infty \text { for all } t \in[0, T]\right\} .
\end{aligned}
$$


Let $\mathcal{G}_{Z}^{ \pm}$be the corresponding classes of functions which respect these integral constraints for each $Z$ :

$$
\mathcal{G}_{Z}^{ \pm}=\left\{g: \int_{-\infty}^{\infty} e^{g(y)} F_{Z}^{ \pm}(y) d y=1 \text { and } \int_{-\infty}^{\infty} y e^{g(y)} F_{Z}^{ \pm}(y) d y<\infty\right\} .
$$

The entropic penalization satisfies:

$$
\begin{aligned}
\mathbb{E}_{t, x, q, S, Z}^{\mathbb{Q}(g)}\left[\mathcal{H}_{t, T}^{\varphi_{\epsilon}}[\mathbb{Q}(g) \mid \mathbb{P}]\right] & =\frac{1}{\varphi_{\epsilon}} \mathbb{E}_{t, x, q, S, Z}^{\mathbb{Q}(g)}\left[\sum_{i=+,-} \int_{t}^{T} \int_{-\infty}^{\infty}\left(e^{g_{u}^{i}(y)}\left(g_{u}^{i}(y)-1\right)+1\right) \nu_{\mathbb{P}}^{i}(d y, d u)\right] \\
& =\frac{1}{\varphi_{\epsilon}} \mathbb{E}_{t, x, q, S, Z}^{\mathbb{Q}(g)}\left[\sum_{i=+,-} \lambda^{i} \int_{t}^{T} \int_{-\infty}^{\infty}\left(e^{g_{u}^{i}(y)}\left(g_{u}^{i}(y)-1\right)+1\right) F_{Z}^{ \pm}(y) d u\right],
\end{aligned}
$$

Under the measure $\mathbb{Q}(g)$, the infinitesimal generator $\mathcal{L}^{\mathbb{Q}(g)}$ is given by:

$$
\begin{aligned}
& \mathcal{L}^{\mathbb{Q}(g)} H^{\epsilon}\left(t, x, q, S, Z ; \varphi_{\epsilon}\right)=\beta^{+} \mathcal{D}_{L}^{+} H^{\epsilon}+\beta^{-} \mathcal{D}_{L}^{-} H^{\epsilon} \\
&+\lambda^{+}(Z) \int_{-\infty}^{\infty} e^{g_{t}^{+}(y)} \mathcal{D}_{M}^{+} H^{\epsilon} F_{Z}^{+}(y) d y \mathbb{1}_{q \neq \underline{q}}+\lambda^{-}(Z) \int_{-\infty}^{\infty} e^{g_{t}^{-}(y)} \mathcal{D}_{M}^{-} H^{\epsilon} F_{Z}^{-}(y) d y \mathbb{1}_{q \neq \bar{q}} \\
&+\sum_{k=1}^{n} G_{Z, k}\left(H^{\epsilon}\left(t, x, q, S, k ; \varphi_{\epsilon}\right)-H^{\epsilon}\left(t, x, q, S, Z ; \varphi_{\epsilon}\right)\right) .
\end{aligned}
$$

Making the standard ansatz, $H^{\epsilon}\left(t, x, q, S, Z ; \varphi_{\epsilon}\right)=x+q S+h_{q, Z}^{\varphi_{\epsilon}}(t)$ and substituting into equation (5.2) gives:

$$
\begin{aligned}
\partial_{t} h_{q, Z}^{\varphi_{\epsilon}}+\beta^{+} \xi^{+} q-\beta^{-} \xi^{-} q & \\
+\sum_{ \pm} \sup _{\gamma^{ \pm} \in\{0,1\}} \inf _{g^{ \pm} \in \mathcal{G}_{Z}^{ \pm}}\left\{\lambda^{ \pm}(Z)\left(\gamma^{ \pm} \frac{\Delta}{2}-\left(\gamma^{ \pm} \mp q\right) \int_{-\infty}^{\infty} y e^{g^{ \pm}(y)} F_{Z}^{ \pm}(y) d y+h_{q \mp \gamma^{ \pm}, Z}^{\varphi_{\epsilon}}-h_{q, Z}^{\varphi_{\epsilon}}\right)\right. & \\
\left.+\frac{\lambda^{ \pm}(Z)}{\varphi_{\epsilon}} \int_{-\infty}^{\infty} e^{g^{ \pm}(y)}\left(g^{ \pm}(y)-1\right)+1 F_{Z}^{ \pm}(y) d y\right\}+\sum_{k=1}^{n} G_{Z, k}\left(h_{q, k}^{\varphi_{\epsilon}}-h_{q, Z}^{\varphi_{\epsilon}}\right) & =0, \\
h_{q, Z}^{\varphi_{\epsilon}}(T) & =-q \ell(q),
\end{aligned}
$$

In the following proposition, it will be assumed that all relevant integrals are finite. The case of what happens when certain integrals become infinite will be discussed in a subsequent proposition.

Proposition 23 (Optimal Feedback Controls I). Suppose $\varphi_{\epsilon}$ is small enough that the 
following two conditions are satisfied for all $q \in\{\underline{q}, \ldots, \bar{q}\}$ :

$$
\begin{gathered}
\int_{-\infty}^{\infty} e^{\mp q \varphi_{\epsilon} y} F_{Z}^{ \pm}(y) d y<\infty \\
\int_{-\infty}^{\infty} y e^{\mp q \varphi_{\epsilon} y} F_{Z}^{ \pm}(y) d y<\infty
\end{gathered}
$$

Such $\varphi_{\epsilon}>0$ exists by the condition in equation (5.6). Then the optimal feedback controls of equation (5.8) are given by:

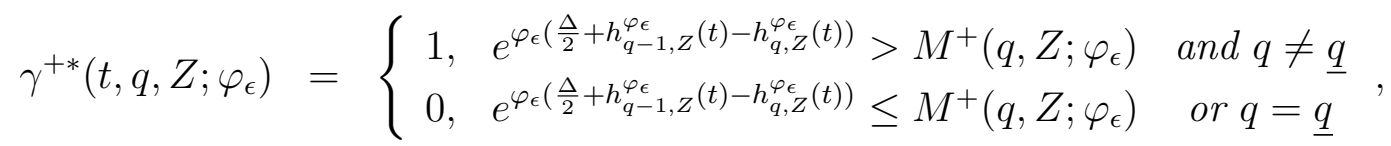

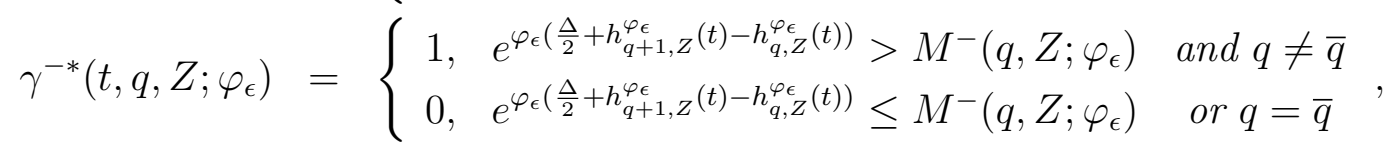

$$
\begin{aligned}
& g^{ \pm *}\left(t, q, Z, y ; \varphi_{\epsilon}\right)=-\log \left(\int_{-\infty}^{\infty} e^{\left(\gamma_{q, Z}^{ \pm *}(t) \mp q\right) \varphi_{\epsilon} y} F^{ \pm}(y) d y\right)+\left(\gamma_{q, Z}^{ \pm *}(t) \mp q\right) \varphi_{\epsilon} y
\end{aligned}
$$

where $M^{ \pm}\left(q, Z ; \varphi_{\epsilon}\right)$ is defined by:

$$
M^{ \pm}\left(q, Z ; \varphi_{\epsilon}\right)=\frac{\int_{-\infty}^{\infty} e^{(1 \mp q) \varphi_{\epsilon} y} F_{Z}^{ \pm}(y) d y}{\int_{-\infty}^{\infty} e^{\mp q \varphi_{\epsilon} y} F_{Z}^{ \pm}(y) d y}
$$

Proof. See Appendix B.4.

\section{Discussion and financial interpretation}

Substituting the expression for $g^{ \pm *}\left(t, q, Z, y ; \varphi_{\epsilon}\right)$ into equation (5.8) results in the following:

$$
\begin{aligned}
& \partial_{t} h_{q, Z}^{\varphi_{\epsilon}}+\beta^{+} \xi^{+} q-\beta^{-} \xi^{-} q \\
&+\sum_{ \pm} \sup _{\gamma^{ \pm} \in\{0,1\}}\left\{\lambda^{ \pm}(Z)\left(\gamma^{ \pm} \frac{\Delta}{2}+h_{q \mp \gamma^{ \pm}, Z}^{\varphi_{\epsilon}}-h_{q, Z}^{\varphi_{\epsilon}}-\frac{1}{\varphi_{\epsilon}} \log \left[\int_{-\infty}^{\infty} e^{\left(\gamma^{ \pm} \mp q\right) \varphi_{\epsilon} y} F_{Z}^{ \pm}(y) d y\right]\right)\right\} \\
&+\sum_{k=1}^{n} G_{Z, k}\left(h_{q, k}^{\varphi_{\epsilon}}-h_{q, Z}^{\varphi_{\epsilon}}\right)=0, \\
& h_{q, Z}^{\varphi_{\epsilon}}(T)=-q \ell(q),
\end{aligned}
$$

Note that for this expression to be valid, $\int_{-\infty}^{\infty} e^{\left(\gamma^{ \pm} \mp q\right) \varphi_{\epsilon} y} F_{Z}^{ \pm}(y) d y$ must be finite. Although the condition in equation (5.6) implies that this is finite for some $\varphi_{\epsilon}>0$, it does not guarantee it for all $\varphi_{\epsilon}>0$. But it is unreasonable to think that an agent's level of 
ambiguity should be constrained by the dynamics of the reference model. The following proposition does not place a restriction on the size of $\varphi_{\epsilon}$, and the result offers a financial interpretation for the how $\varphi_{\epsilon}$ affects the agent's optimal strategy.

Proposition 24 (Optimal Feedback Controls II). The optimal feedback controls of equation (5.8) are given by:

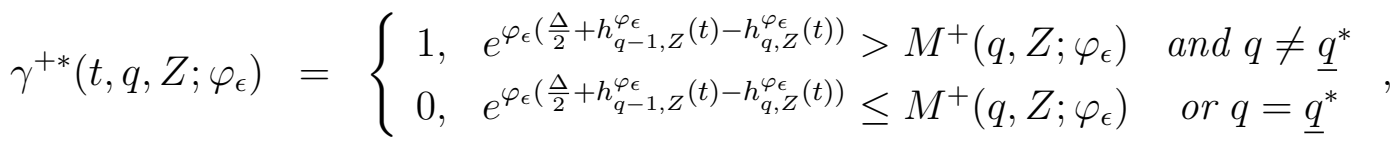

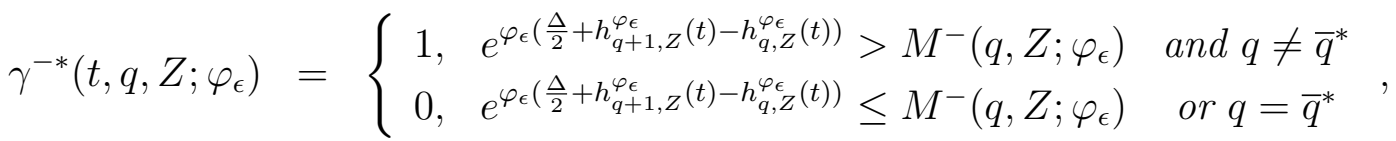

$$
\begin{aligned}
& g^{ \pm *}\left(t, q, Z, y ; \varphi_{\epsilon}\right)=\left\{\begin{array}{cc}
k^{ \pm *}\left(t, q, Z, y ; \varphi_{\epsilon}\right) & \text { for } q \in\left\{\underline{q}^{*}, \ldots, \bar{q}^{*}\right\} \\
\text { undefined } & \text { for } q \notin\left\{\underline{q}^{*}, \ldots, \bar{q}^{*}\right\}
\end{array}\right. \text {, }
\end{aligned}
$$

where $k^{ \pm *}\left(t, q, Z, y ; \varphi_{\epsilon}\right)$ is defined by:

$$
k^{ \pm *}\left(t, q, Z, y ; \varphi_{\epsilon}\right)=-\log \left(\int_{-\infty}^{\infty} e^{\left(\gamma_{q, Z}^{ \pm *}(t) \mp q\right) \varphi_{\epsilon} y} F^{ \pm}(y) d y\right)+\left(\gamma_{q, Z}^{ \pm *}(t) \mp q\right) \varphi_{\epsilon} y
$$

$M^{ \pm}\left(q, Z ; \varphi_{\epsilon}\right)$ is defined by:

$$
M^{ \pm}\left(q, Z ; \varphi_{\epsilon}\right)=\frac{\int_{-\infty}^{\infty} e^{(1 \mp q) \varphi_{\epsilon} y} F_{Z}^{ \pm}(y) d y}{\int_{-\infty}^{\infty} e^{\mp q \varphi_{\epsilon} y} F_{Z}^{ \pm}(y) d y}
$$

and $\underline{q}^{*}$ and $\bar{q}^{*}$ are defined by:

$$
\begin{aligned}
& \underline{q}^{*}=\min \left\{q \geq \underline{q}: \int_{-\infty}^{\infty} e^{\mp q \varphi_{\epsilon} y} F_{Z}^{ \pm}(y) d y<\infty \text { and } \Upsilon^{ \pm}\left(q, Z ; \varphi_{\epsilon}\right)<\infty \text { for } Z \in\{1, \ldots, n\}\right\} \\
& \bar{q}^{*}=\max \left\{q \leq \bar{q}: \int_{-\infty}^{\infty} e^{\mp q \varphi_{\epsilon} y} F_{Z}^{ \pm}(y) d y<\infty \text { and } \Upsilon^{ \pm}\left(q, Z ; \varphi_{\epsilon}\right)<\infty \text { for } Z \in\{1, \ldots, n\}\right\}
\end{aligned}
$$

where $\Upsilon^{ \pm}\left(q, Z ; \varphi_{\epsilon}\right)$ is defined by:

$$
\Upsilon^{ \pm}\left(q, Z ; \varphi_{\epsilon}\right)=\lim _{\phi \rightarrow \varphi_{\epsilon}}\left(1-\frac{\phi}{\varphi_{\epsilon}}\right) \frac{\int_{-\infty}^{\infty} y e^{\mp q \phi y} F_{Z}^{ \pm}(y) d y}{\int_{-\infty}^{\infty} e^{\mp q \phi y} F_{Z}^{ \pm}(y) d y}
$$

Proof. See Appendix B.4.

There is no difference between the form of the feedback controls in Propositions 23 
and 24, only the values of $q$ for which the choice of $\gamma^{ \pm}=0$ is necessary are changed. The interpretation of this result is the following: if the ambiguity level $\varphi_{\epsilon}$ does not satisfy the constraints in (5.9) and (5.10) for all $q \in\{\underline{q}, \ldots, \bar{q}\}$ and $Z \in\{1, \ldots, n\}$ because it is too large, then the minimum and maximum allowable inventory levels are redefined to a smaller interval until these constraints are satisfied. The agent's fear of an unknown distribution of jump sizes is so great that they become infinitely averse to holding large values of inventory. This change of allowable inventory levels is not a matter of mathematical convenience to avoid infinities - it is a matter of the agent's desire to avoid states where their value function is $-\infty$. As shown in the proof of Proposition 24, outside of this redefined inventory range, the value of the function $h_{q, Z}^{\varphi_{\epsilon}}(t)$ is $-\infty$. Also of noteworthy importance is that the redefined upper and lower inventory values depend on the reference distributions $F_{Z}^{ \pm}(y)$ for all $Z \in\{1, \ldots, n\}$ and for both market buy and sell orders.

In order to perform any numerical computations, a specific form of $F_{Z}^{ \pm}(y)$ will be specified. For the remainder of this section, choose:

$$
F_{Z}^{ \pm}(y)=\kappa_{Z}^{ \pm} e^{-\kappa_{Z}^{ \pm} y} \mathbb{1}_{y \geq 0}
$$

so that the distribution of jump sizes after market orders is exponential, with parameter depending on the imbalance state. With this distribution, the feedback expression for $g^{ \pm *}\left(t, q, Z, y ; \varphi_{\epsilon}\right)$ only gives finite values for $\left(\gamma^{ \pm} \mp q\right) \varphi_{\epsilon}-\kappa_{Z}^{ \pm}<0$, and the expression simplifies to:

$$
g^{ \pm *}\left(t, q, Z, y ; \varphi_{\epsilon}\right)=-\log \left(\frac{-\kappa_{Z}^{ \pm}}{\left(\gamma^{ \pm} \mp q\right) \varphi_{\epsilon}-\kappa_{Z}^{ \pm}}\right)+\left(\gamma^{ \pm} \mp q\right) \varphi_{\epsilon} y
$$

The corresponding differential equation of interest is:

$$
\begin{aligned}
& \partial_{t} h_{q, Z}^{\varphi_{\epsilon}}+\beta^{+} \xi^{+} q-\beta^{-} \xi^{-} q \\
&+\sum_{ \pm} \sup _{\gamma^{ \pm} \in\{0,1\}}\left\{\lambda^{ \pm}(Z)\left(\gamma^{ \pm} \frac{\Delta}{2}+h_{q \mp \gamma^{ \pm}, Z}^{\varphi_{\epsilon}}-h_{q, Z}^{\varphi_{\epsilon}}-\frac{1}{\varphi_{\epsilon}} \log \left[\frac{\kappa_{Z}^{ \pm}}{\kappa_{Z}^{ \pm}-\left(\gamma^{ \pm} \mp q\right) \varphi_{\epsilon}}\right]\right)\right\} \\
&+\sum_{k=1}^{n} G_{Z, k}\left(h_{q, k}^{\varphi_{\epsilon}}-h_{q, Z}^{\varphi_{\epsilon}}\right)=0, \\
& h_{q, Z}^{\varphi_{\epsilon}}(T)=-q \ell(q),
\end{aligned}
$$

For this choice of jump distribution, the constraint $\mp q \varphi_{\epsilon}-\kappa_{Z}^{ \pm}<0$ must be satisfied to achieve finite values. This is how $\underline{q}^{*}$ and $\bar{q}^{*}$ of Proposition 24 are chosen. 


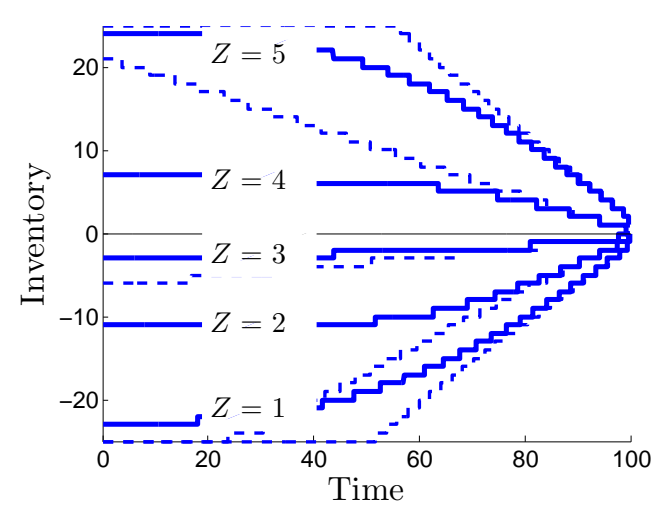

(a) Sell Boundary

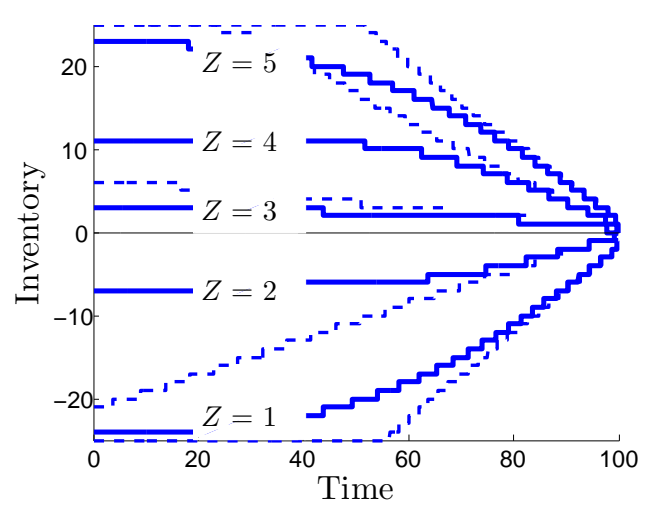

(b) Buy Boundary

Figure 5.2: Trade boundaries for a market maker that is ambiguity averse to the distribution of midprice jumps following market orders (dashed lines are ambiguity neutral boundaries). Additional parameters are $\varphi_{\epsilon}=0.2, T=100, \ell(q)=-\theta q, \theta=0.005$, $\bar{q}=-\underline{q}=25$.

\subsection{Ambiguity aversion with respect to imbalance regime changes}

In this section, the standard method for incorporating ambiguity aversion will be conducted with respect to the generator matrix, $G$, of the imbalance regime process. The technical details of this process must be expanded before the proper class of measure changes and corresponding penalization can be introduced. To this end, introduce a doubly stochastic Poisson random measure $\pi(d s, d t)$ with compensator $\rho_{t}^{\mathbb{P}}(d s, d t)=$ $\sum_{k \neq Z_{t}} G_{Z_{t}, k} \delta_{k}(s) d s d t$. Recall that $G_{Z, k}$ are the elements of the generator matrix of the process $Z_{t}$, and $\delta_{k}$ is the Dirac delta functional centred at $k$. This doubly stochastic Poisson random measure exists on the state space of the imbalance regime process, $\mathcal{Z}=\{1, \ldots, n\}$. Now define $Z_{t}$ to satisfy:

$$
d Z_{t}=\int_{\mathcal{Z}}<Z_{t^{-}}, s>\pi(d s, d t)
$$

where $\langle a, b\rangle$ is an element of the transition groupoid representing transition from regime $a$ to regime $b$. This definition of $Z_{t}$ will result in the same dynamics as that of the reference model (see Appendix A.3 for more details). This definition also allows for the appropriate class of Radon-Nikodym derivatives and entropic penalizations to be easily 
defined:

$$
\frac{d \mathbb{Q}(g)}{d \mathbb{P}}=\exp \left\{-\int_{0}^{T} \int_{\mathcal{Z}}\left(e^{g_{t}(s)}-1\right) \rho_{t}^{\mathbb{P}}(d s, d t)+\int_{0}^{T} \int_{\mathcal{Z}} g_{t}(s) \pi(d s, d t)\right\}
$$

The full class of candidate measures is selected to be:

$$
\mathcal{Q}^{G}=\left\{\mathbb{Q}(g): g \text { are } \mathcal{F}_{t} \text {-predictable, Markov, and bounded }\right\} .
$$

In the measure $\mathbb{Q}(g)$, the compensator of $\pi(d s, d t)$ is $\rho_{Z}^{\mathbb{Q}}(d s, d t)=e^{g_{t}(s)} \rho_{Z}^{\mathbb{P}}(d s, d t)=$ $\sum_{k \neq Z_{t}} e^{g_{t}(s)} G_{Z_{t}, k} \delta_{k}(s) d s d t$. The entropic penalization satisfies in this case:

$$
\begin{aligned}
\mathbb{E}_{t, x, q, S, Z}^{\mathbb{Q}(g)}\left[\mathcal{H}_{t, T}^{\varphi_{G}}[\mathbb{Q}(g) \mid \mathbb{P}]\right] & =\frac{1}{\varphi_{G}} \mathbb{E}_{t, x, q, S, Z}^{\mathbb{Q}(g)}\left[\int_{t}^{T} \int_{\mathcal{Z}}\left(e^{g_{u}(s)}\left(g_{u}(s)-1\right)+1\right) \rho_{u}^{\mathbb{P}}(d s, d u)\right] \\
& =\frac{1}{\varphi_{G}} \mathbb{E}_{t, x, q, S, Z}^{\mathbb{Q}(g)}\left[\int_{t}^{T} \int_{\mathcal{Z}}\left(e^{g_{u}(s)}\left(g_{u}(s)-1\right)+1\right) \sum_{k \neq Z_{u}} G_{Z_{u}, k} \delta_{k}(s) d s d u\right] \\
& =\frac{1}{\varphi_{G}} \mathbb{E}_{t, x, q, S, Z}^{\mathbb{Q}(g)}\left[\int_{t}^{T} \int_{\mathcal{Z}} \sum_{k \neq Z_{u}}\left(e^{g_{u}(s)}\left(g_{u}(s)-1\right)+1\right) G_{Z_{u}, k} \delta_{k}(s) d s d u\right] \\
& =\frac{1}{\varphi_{G}} \mathbb{E}_{t, x, q, S, Z}^{\mathbb{Q}(g)}\left[\int_{t}^{T} \sum_{k \neq Z_{u}}\left(e^{g_{u}(k)}\left(g_{u}(k)-1\right)+1\right) G_{Z_{u}, k} d u\right]
\end{aligned}
$$

Under the measure $\mathbb{Q}(g)$, the infinitesimal generator $\mathcal{L}^{\mathbb{Q}(g)}$ is given by:

$$
\begin{aligned}
\mathcal{L}^{\mathbb{Q}(g)} H^{G}\left(t, x, q, S, Z ; \varphi_{G}\right) & =\beta^{+} \mathcal{D}_{L}^{+} H^{G}+\beta^{-} \mathcal{D}_{L}^{-} H^{G} \\
+\lambda^{+}(Z) \int_{-\infty}^{\infty} & \mathcal{D}_{M}^{+} H^{G} F_{Z}^{+}(y) d y \mathbb{1}_{q \neq \underline{q}}+\lambda^{-}(Z) \int_{-\infty}^{\infty} \mathcal{D}_{M}^{-} H^{G} F_{Z}^{-}(y) d y \mathbb{1}_{q \neq \bar{q}} \\
& \quad+\sum_{k=1}^{n} e^{g_{t}(k)} G_{Z, k}\left(H^{G}\left(t, x, q, S, k ; \varphi_{G}\right)-H^{G}\left(t, x, q, S, Z ; \varphi_{G}\right)\right) .
\end{aligned}
$$


Making the standard ansatz, $H^{G}\left(t, x, q, S, Z ; \varphi_{G}\right)=x+q S+h_{q, Z}^{\varphi_{G}}(t)$ and substituting into equation (5.2) gives:

$$
\begin{aligned}
& \partial_{t} h_{q, Z}^{\varphi_{G}}+\beta^{+} \xi^{+} q-\beta^{-} \xi^{-} q \\
&+\sum_{ \pm} \sup _{\gamma^{ \pm} \in\{0,1\}} \inf _{g}\left\{\lambda^{ \pm}(Z)\left(\gamma^{ \pm} \frac{\Delta}{2}-\left(\gamma^{ \pm} \mp q\right) \epsilon_{Z}^{ \pm}+h_{q \mp \gamma^{ \pm}, Z}^{\varphi_{G}}-h_{q, Z}^{\varphi_{G}}\right)\right. \\
&\left.+\sum_{k=1}^{n} e^{g(k)} G_{Z, k}\left(h_{q, k}^{\varphi_{G}}-h_{q, Z}^{\varphi_{G}}\right)+\frac{1}{\varphi_{G}} \sum_{k \neq Z}\left(e^{g(k)}(g(k)-1)+1\right) G_{Z, k}\right\}=0, \\
& h_{q, Z}^{\varphi_{G}}(T)=-q \ell(q),
\end{aligned}
$$

Proposition 25 (Optimal Feedback Controls). The optimal feedback controls of equation (5.13) are given by:

$$
\begin{aligned}
& \gamma^{+*}\left(t, q, Z ; \varphi_{G}\right)=\left\{\begin{array}{c}
1, \quad \frac{\Delta}{2}-\epsilon_{Z}^{+}+h_{q-1, Z}^{\varphi_{G}}(t)-h_{q, Z}^{\varphi_{G}}(t)>0 \quad \text { and } q \neq \underline{q} \\
0, \quad \frac{\Delta}{2}-\epsilon_{Z}^{+}+h_{q-1, Z}^{\varphi_{G}}(t)-h_{q, Z}^{\varphi_{G}}(t) \leq 0 \quad \text { or } q=\underline{q}
\end{array}\right. \\
& \gamma^{-*}\left(t, q, Z ; \varphi_{G}\right)=\left\{\begin{array}{c}
1, \quad \frac{\Delta}{2}-\epsilon_{Z}^{-}+h_{q+1, Z}^{\varphi_{G}}(t)-h_{q, Z}^{\varphi_{G}}(t)>0 \quad \text { and } q \neq \bar{q} \\
0, \quad \frac{\Delta}{2}-\epsilon_{Z}^{-}+h_{q+1, Z}^{\varphi_{G}}(t)-h_{q, Z}^{\varphi_{G}}(t) \leq 0 \quad \text { or } q=\bar{q}
\end{array}\right. \\
& g^{*}\left(t, q, Z, s ; \varphi_{G}\right)=-\varphi_{G}\left(h_{q, k}^{\varphi_{G}}(t)-h_{z, Z}^{\varphi_{G}}(t)\right)
\end{aligned}
$$

Proof. See Appendix B.4.

\section{Discussion and financial interpretation}

Substituting the optimal feedback expression for $g^{*}\left(t, q, Z, s ; \varphi_{G}\right)$ into equation (5.13) gives an equation which is easily solved numerically:

$$
\begin{aligned}
\partial_{t} h_{q, Z}^{\varphi_{G}}+\beta^{+} \xi^{+} q-\beta^{-} \xi^{-} q & \\
+\sum_{ \pm} \sup _{\gamma^{ \pm} \in\{0,1\}}\left\{\lambda^{ \pm}(Z)\left(\gamma^{ \pm} \frac{\Delta}{2}-\left(\gamma^{ \pm} \mp q\right) \epsilon_{Z}^{ \pm}+h_{q \mp \gamma^{ \pm}, Z}^{\varphi_{G}}-h_{q, Z}^{\varphi_{G}}\right)\right\} & \\
+\frac{1}{\varphi_{G}} \sum_{k \neq Z}\left(1-e^{-\varphi_{G}\left(h_{q, k}^{\varphi_{G}}-h_{q, Z}^{\varphi_{G}}\right)}\right) G_{Z, k} & =0, \quad(5.14) \\
h_{q, Z}^{\varphi_{G}}(T) & =-q \ell(q),
\end{aligned}
$$

Solving this equation numerically results in the trade boundaries shown in Figure 5.3. 


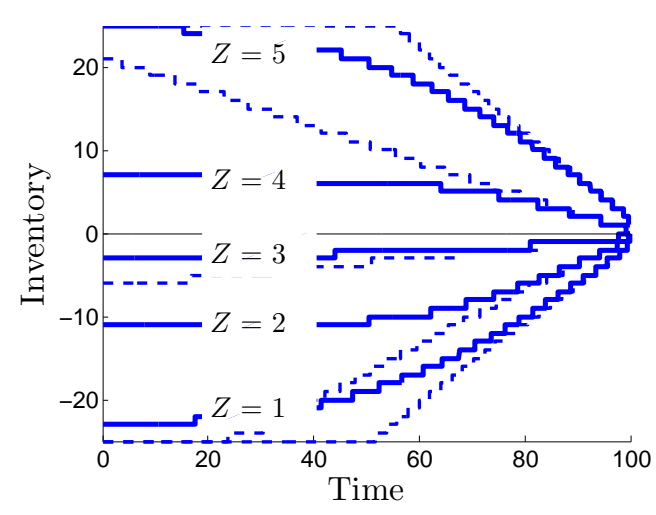

(a) Sell Boundary

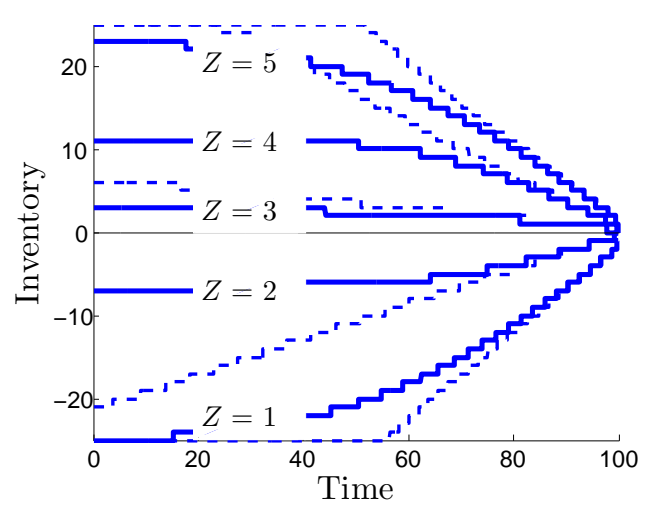

(b) Buy Boundary

Figure 5.3: Trade boundaries for a market maker that is ambiguity averse to imbalance regime changes (dashed lines are ambiguity neutral boundaries). Additional parameters are $\varphi_{G}=0.1, T=100, \ell(q)=-\theta q, \theta=0.005, \bar{q}=-\underline{q}=25$.

At this point, ambiguity aversion has been considered with respect to three aspects of the model: the rate of market order arrivals, the distribution of midprice jumps, and the generator matrix associated with the imbalance regime process. The generator matrix in essence encapsulates two different types of quantities that fully describe the evolution of the imbalance regime process: the rate at which the process exits from each regime and the probability of entering all other regimes at the instant of a regime switch. The market maker could have different levels of ambiguity with respect to both of these quantities (possibly arising from having more confidence in certain stages of the calibration process outlined in Section 4.5). The next sections will investigate the effects of ambiguity aversion on these factors of the model separately.

\subsubsection{Transition rates}

Ambiguity aversion with respect to only the transition rates out of an imbalance regime state is handled in the same way as ambiguity on the entire generator matrix with the added constraint that the transition probabilities to other states (conditional on a transition event occuring) remain fixed. This is accomplished in a similar fashion as other cases where only the rate is changed: the process $g_{t}$ corresponding to the measure change is allowed to only depend on $t$, not on the spacial variable. Then only the time dependence of $\rho_{t}^{\mathbb{P}}(d s, d t)$ will be affected. The form of measure changes to be used in this 
case then is:

$$
\frac{d \mathbb{Q}(g)}{d \mathbb{P}}=\exp \left\{-\int_{0}^{T} \int_{\mathcal{Z}}\left(e^{g_{t}}-1\right) \rho_{t}^{\mathbb{P}}(d s, d t)+\int_{0}^{T} \int_{\mathcal{Z}} g_{t} \pi(d s, d t)\right\},
$$

and the full class of candidate measures is selected to be:

$$
\mathcal{Q}^{R}=\left\{\mathbb{Q}(g): g \text { are } \mathcal{F}_{t} \text {-predictable, Markov, and bounded }\right\} .
$$

In the measure $\mathbb{Q}(g)$, the compensator of $\pi(d s, d t)$ is $\rho_{t}^{\mathbb{Q}}(d s, d t)=e^{g t} \rho_{t}^{\mathbb{P}}(d s, d t)$. The entropic penalization satisfies in this case:

$$
\begin{aligned}
\mathbb{E}_{t, x, q, S, Z}^{\mathbb{Q}(g)}\left[\mathcal{H}_{t, T}^{\varphi_{R}}[\mathbb{Q}(g) \mid \mathbb{P}]\right] & =\frac{1}{\varphi_{R}} \mathbb{E}_{t, x, q, S, Z}^{\mathbb{Q}(g)}\left[\int_{t}^{T} \int_{\mathcal{Z}}\left(e^{g_{u}}\left(g_{u}-1\right)+1\right) \rho_{u}^{\mathbb{P}}(d s, d u)\right] \\
& =\frac{1}{\varphi_{R}} \mathbb{E}_{t, x, q, S, Z}^{\mathbb{Q}(g)}\left[\int_{t}^{T} \int_{\mathcal{Z}}\left(e^{g_{u}}\left(g_{u}-1\right)+1\right) \sum_{k \neq Z_{u}} G_{Z_{u}, k} \delta_{k}(s) d s d u\right] \\
& =\frac{1}{\varphi_{R}} \mathbb{E}_{t, x, q, S, Z}^{\mathbb{Q}(g)}\left[\int_{t}^{T}\left(e^{g_{u}}\left(g_{u}-1\right)+1\right) \sum_{k \neq Z_{u}} G_{Z_{u}, k} d u\right]
\end{aligned}
$$

Under the measure $\mathbb{Q}(g)$, the infinitesimal generator $\mathcal{L}^{\mathbb{Q}(g)}$ is given by:

$$
\begin{aligned}
\mathcal{L}^{\mathbb{Q}(g)} H^{R}\left(t, x, q, S, Z ; \varphi_{G}\right)= & \beta^{+} \mathcal{D}_{L}^{+} H^{R}+\beta^{-} \mathcal{D}_{L}^{-} H^{R} \\
+\lambda^{+}(Z) \int_{-\infty}^{\infty} & \mathcal{D}_{M}^{+} H^{R} F_{Z}^{+}(y) d y \mathbb{1}_{q \neq \underline{q}}+\lambda^{-}(Z) \int_{-\infty}^{\infty} \mathcal{D}_{M}^{-} H^{R} F_{Z}^{-}(y) d y \mathbb{1}_{q \neq \bar{q}} \\
& +e^{g_{t}} \sum_{k=1}^{n} G_{Z, k}\left(H^{R}\left(t, x, q, S, k ; \varphi_{R}\right)-H^{R}\left(t, x, q, S, Z ; \varphi_{R}\right)\right) .
\end{aligned}
$$

Making the standard ansatz, $H^{R}\left(t, x, q, S, Z ; \varphi_{R}\right)=x+q S+h_{q, Z}^{\varphi_{R}}(t)$ and substituting into equation (5.2) gives:

$$
\begin{aligned}
\partial_{t} h_{q, Z}^{\varphi_{R}}+\beta^{+} \xi^{+} q-\beta^{-} \xi^{-} q & \\
+\sum_{ \pm} \sup _{\gamma^{ \pm} \in\{0,1\}} \inf _{g}\left\{\lambda^{ \pm}(Z)\left(\gamma^{ \pm} \frac{\Delta}{2}-\left(\gamma^{ \pm} \mp q\right) \epsilon_{Z}^{ \pm}+h_{q \mp \gamma^{ \pm}, Z}^{\varphi_{R}}-h_{q, Z}^{\varphi_{R}}\right)\right. & \\
\left.+e^{g} \sum_{k=1}^{n} G_{Z, k}\left(h_{q, k}^{\varphi_{R}}-h_{q, Z}^{\varphi_{R}}\right)+\frac{1}{\varphi_{R}}\left(e^{g}(g-1)+1\right) \sum_{k \neq Z} G_{Z, k}\right\} & =0, \quad(5.15) \\
h_{q, Z}^{\varphi_{R}}(T) & =-q \ell(q),
\end{aligned}
$$

Proposition 26 (Optimal Feedback Controls). The optimal feedback controls of equation 
(5.15) are given by:

$$
\begin{aligned}
& \gamma^{+*}\left(t, q, Z ; \varphi_{R}\right)=\left\{\begin{array}{ll}
1, & \frac{\Delta}{2}-\epsilon_{Z}^{+}+h_{q-1, Z}^{\varphi_{R}}(t)-h_{q, Z}^{\varphi_{R}}(t)>0 \quad \text { and } q \neq \underline{q} \\
0, & \frac{\Delta}{2}-\epsilon_{Z}^{+}+h_{q-1, Z}^{\varphi_{R}}(t)-h_{q, Z}^{\varphi_{R}}(t) \leq 0 \quad \text { or } q=\underline{q}
\end{array},\right. \\
& \gamma^{-*}\left(t, q, Z ; \varphi_{R}\right)=\left\{\begin{array}{ccc}
1, & \frac{\Delta}{2}-\epsilon_{Z}^{-}+h_{q+1, Z}^{\varphi_{R}}(t)-h_{q, Z}^{\varphi_{R}}(t)>0 \quad \text { and } q \neq \bar{q} \\
0, & \frac{\Delta}{2}-\epsilon_{Z}^{-}+h_{q+1, Z}^{\varphi_{R}}(t)-h_{q, Z}^{\varphi_{R}}(t) \leq 0 \quad \text { or } q=\bar{q}
\end{array},\right. \\
& g^{*}\left(t, q, Z, s ; \varphi_{R}\right)=-\varphi_{R} \frac{\left(\sum_{k=1}^{n} G_{Z, k}\left(h_{q, k}^{\varphi_{R}}(t)-h_{q, Z}^{\varphi_{R}}(t)\right)\right)}{\sum_{k \neq Z} G_{Z, k}}
\end{aligned}
$$

Proof. See Appendix B.4.

\section{Discussion and financial interpretation}

Substituting the optimal feedback expression for $g^{*}\left(t, q, Z, s ; \varphi_{R}\right)$ into equation (5.15) gives an equation which is easily solved numerically:

$$
\begin{aligned}
& \partial_{t} h_{q, Z}^{\varphi_{R}}+\beta^{+} \xi^{+} q-\beta^{-} \xi^{-} q \\
&+\sum_{ \pm} \sup _{\gamma^{ \pm} \in\{0,1\}}\left\{\lambda^{ \pm}(Z)\left(\gamma^{ \pm} \frac{\Delta}{2}-\left(\gamma^{ \pm} \mp q\right) \epsilon_{Z}^{ \pm}+h_{q \neq \gamma^{ \pm}, Z}^{\varphi_{R}}-h_{q, Z}^{\varphi_{R}}\right)\right\} \\
&+\frac{\sum_{k \neq Z} G_{Z, k}}{\varphi_{R}}\left(1-\exp \left\{-\varphi_{R} \frac{\sum_{k \neq Z} G_{Z, k}\left(h_{q, k}^{\varphi_{R}}-h_{q, Z}^{\varphi_{R}}\right)}{\sum_{k \neq Z} G_{Z, k}}\right\}\right)=0, \quad(5.16) \\
& h_{q, Z}^{\varphi_{R}}(T)=-q \ell(q),
\end{aligned}
$$

\subsubsection{Transition probabilities}

The market maker may be confident in the model with respect to how quickly the imbalance regime changes states, but lack confidence in the probabilities that it changes to other states conditional on a change occurring. In this case, they are only ambiguity averse to these transition probabilities, and the class of measure changes must be selected appropriately to make sure the rate at which regime switches happen remains fixed. This is done by putting restrictions on the possible compensators in the new measure. The 


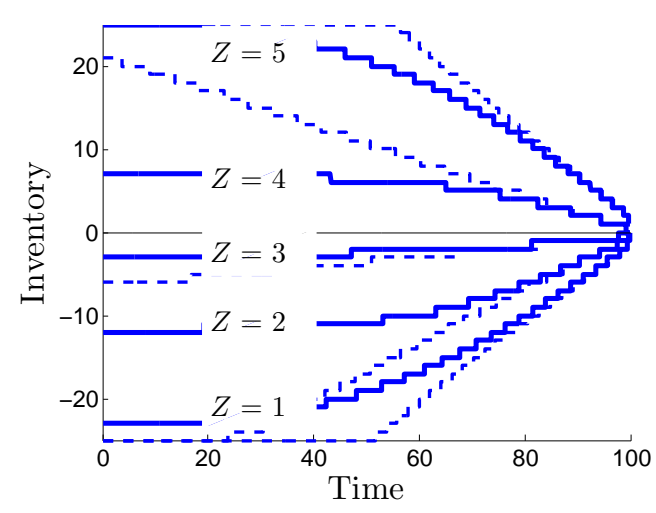

(a) Sell Boundary

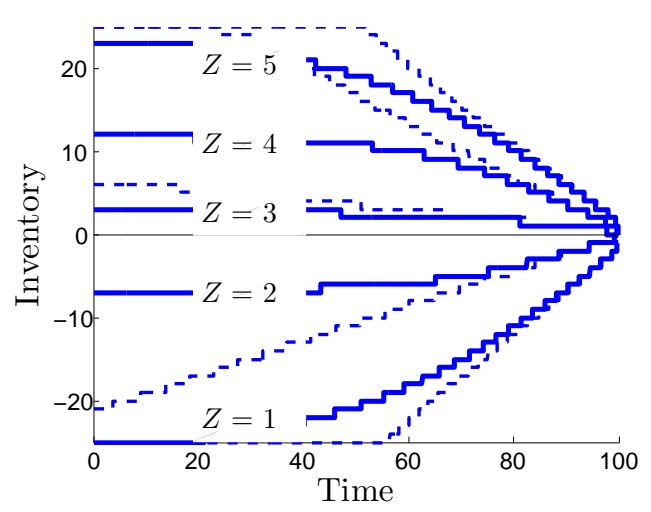

(b) Buy Boundary

Figure 5.4: Trade boundaries for a market maker that is ambiguity averse to rate of imbalance regime changes (dashed lines are ambiguity neutral boundaries). Additional parameters are $\varphi_{R}=0.2, T=100, \ell(q)=-\theta q, \theta=0.005, \bar{q}=-\underline{q}=25$.

Radon-Nikodym derivatives that generate the candidate measures are given by:

$$
\frac{d \mathbb{Q}(g)}{d \mathbb{P}}=\exp \left\{-\int_{0}^{T} \int_{\mathcal{Z}}\left(e^{g_{t}(s)}-1\right) \rho_{t}^{\mathbb{P}}(d s, d t)+\int_{0}^{T} \int_{\mathcal{Z}} g_{t}(s) \pi(d s, d t)\right\}
$$

The full class of candidate measures is selected to be:

$$
\begin{gathered}
\mathcal{Q}^{P}=\left\{\mathbb{Q}(g): g \text { are } \mathcal{F}_{t^{-} \text {-predictable, Markov, bounded, and satisfy }}\right. \\
\left.\sum_{k \neq Z_{t}} e^{g_{t}(k)} G_{Z_{t}, k}=\sum_{k \neq Z_{t}} G_{Z_{t}, k} \text { for all } t \in[0, T]\right\} .
\end{gathered}
$$

In the measure $\mathbb{Q}(g)$, the compensator of $\pi(d s, d t)$ is $\rho_{t}^{\mathbb{Q}}(d s, d t)=e^{g_{t}(s)} \rho_{t}^{\mathbb{P}}(d s, d t)=$ $\sum_{k \neq Z_{t}} e^{g_{t}(s)} G_{Z_{t}, k} \delta_{k}(s) d s d t$. Let $\mathcal{G}_{Z}$ be the corresponding classes of functions which respect these integral constraints for each $Z$ :

$$
\mathcal{G}_{Z}=\left\{g: \sum_{k \neq Z} e^{g(k)} G_{Z, k}=\sum_{k \neq Z} G_{Z, k}\right\}
$$


The entropic penalization satisfies in this case:

$$
\begin{aligned}
\mathbb{E}_{t, x, q, S, Z}^{\mathbb{Q}(g)}\left[\mathcal{H}_{t, T}^{\varphi_{P}}[\mathbb{Q}(g) \mid \mathbb{P}]\right] & =\frac{1}{\varphi_{P}} \mathbb{E}_{t, x, q, S, Z}^{\mathbb{Q}(g)}\left[\int_{t}^{T} \int_{\mathcal{Z}}\left(e^{g_{u}(s)}\left(g_{u}(s)-1\right)+1\right) \rho_{Z_{u}}^{\mathbb{P}}(d s, d u)\right] \\
& =\frac{1}{\varphi_{P}} \mathbb{E}_{t, x, q, S, Z}^{\mathbb{Q}(g)}\left[\int_{t}^{T} \int_{\mathcal{Z}}\left(e^{g_{u}(s)}\left(g_{u}(s)-1\right)+1\right) \sum_{k \neq Z_{u}} G_{Z_{u}, k} \delta_{k}(s) d s d u\right] \\
& =\frac{1}{\varphi_{P}} \mathbb{E}_{t, x, q, S, Z}^{\mathbb{Q}(g)}\left[\int_{t}^{T} \int_{\mathcal{Z}} \sum_{k \neq Z_{u}}\left(e^{g_{u}(s)}\left(g_{u}(s)-1\right)+1\right) G_{Z_{u}, k} \delta_{k}(s) d s d u\right] \\
& =\frac{1}{\varphi_{P}} \mathbb{E}_{t, x, q, S, Z}^{\mathbb{Q}(g)}\left[\int_{t}^{T} \sum_{k \neq Z_{u}}\left(e^{g_{u}(k)}\left(g_{u}(k)-1\right)+1\right) G_{Z_{u}, k} d u\right]
\end{aligned}
$$

The infinitesimal generator $\mathcal{L}^{\mathbb{Q}(g)}$ is given by:

$$
\begin{aligned}
\mathcal{L}^{\mathbb{Q}(g)} H^{P}\left(t, x, q, S, Z ; \varphi_{P}\right) & =\beta^{+} \mathcal{D}_{L}^{+} H^{P}+\beta^{-} \mathcal{D}_{L}^{-} H^{P} \\
& +\lambda^{+}(Z) \int_{-\infty}^{\infty} \mathcal{D}_{M}^{+} H^{P} F_{Z}^{+}(y) d y \mathbb{1}_{q \neq \underline{q}}+\lambda^{-}(Z) \int_{-\infty}^{\infty} \mathcal{D}_{M}^{-} H^{P} F_{Z}^{-}(y) d y \mathbb{1}_{q \neq \bar{q}} \\
& +\sum_{k=1}^{n} e^{g_{t}(k)} G_{Z, k}\left(H^{P}\left(t, x, q, S, k ; \varphi_{P}\right)-H^{P}\left(t, x, q, S, Z ; \varphi_{P}\right)\right) .
\end{aligned}
$$

Making the standard ansatz, $H^{P}\left(t, x, q, S, Z ; \varphi_{P}\right)=x+q S+h_{q, Z}^{\varphi_{P}}(t)$ and substituting into equation (5.2) gives:

$$
\begin{aligned}
& \partial_{t} h_{q, Z}^{\varphi_{P}}+\beta^{+} \xi^{+} q-\beta^{-} \xi^{-} q \\
&+\sum_{ \pm} \sup _{\gamma^{ \pm} \in\{0,1\}} \inf _{g \in \mathcal{G}_{Z}}\left\{\lambda^{ \pm}(Z)\left(\gamma^{ \pm} \frac{\Delta}{2}-\left(\gamma^{ \pm} \mp q\right) \epsilon_{Z}^{ \pm}+h_{q \mp \gamma^{ \pm}, Z}^{\varphi_{P}}-h_{q, Z}^{\varphi_{P}}\right)\right. \\
&\left.+\sum_{k=1}^{n} e^{g(k)} G_{Z, k}\left(h_{q, k}^{\varphi_{P}}-h_{q, Z}^{\varphi_{P}}\right)+\frac{1}{\varphi_{P}} \sum_{k \neq Z}\left(e^{g(k)}(g(k)-1)+1\right) G_{Z, k}\right\}=0, \quad(5.1) \\
& h_{q, Z}^{\varphi_{P}}(T)=-q \ell(q),
\end{aligned}
$$

Proposition 27 (Optimal Feedback Controls). The optimal feedback controls of equation 
(5.17) are given by:

$$
\begin{aligned}
& \gamma^{+*}\left(t, q, Z ; \varphi_{P}\right)=\left\{\begin{array}{c}
1, \quad \frac{\Delta}{2}-\epsilon_{Z}^{+}+h_{q-1, Z}^{\varphi_{P}}(t)-h_{q, Z}^{\varphi_{P}}(t)>0 \quad \text { and } q \neq \underline{q} \\
0, \quad \frac{\Delta}{2}-\epsilon_{Z}^{+}+h_{q-1, Z}^{\varphi_{P}}(t)-h_{q, Z}^{\varphi_{P}}(t) \leq 0 \quad \text { or } q=\underline{q}
\end{array}\right. \\
& \gamma^{-*}\left(t, q, Z ; \varphi_{P}\right)=\left\{\begin{array}{cc}
1, & \frac{\Delta}{2}-\epsilon_{Z}^{-}+h_{q+1, Z}^{\varphi_{P}}(t)-h_{q, Z}^{\varphi_{P}}(t)>0 \quad \text { and } q \neq \bar{q} \\
0, & \frac{\Delta}{2}-\epsilon_{Z}^{-}+h_{q+1, Z}^{\varphi_{P}}(t)-h_{q, Z}^{\varphi_{P}}(t) \leq 0 \quad \text { or } q=\bar{q}
\end{array},\right. \\
& g^{*}\left(t, q, Z, s ; \varphi_{P}\right)=-\log \left(\frac{\sum_{k \neq Z} G_{Z, k} e^{-\varphi_{P}\left(h_{q, k}^{\varphi_{P}}(t)-h_{q, Z}^{\varphi_{P}}(t)\right)}}{\sum_{k \neq Z} G_{Z, k}}\right)-\varphi_{P}\left(h_{q, s}^{\varphi_{P}}(t)-h_{q, Z}^{\varphi_{P}}(t)\right)
\end{aligned}
$$

Proof. See Appendix B.4.

\section{Discussion and financial interpretation}

Substituting the expression for $g^{*}\left(t, q, Z, s ; \varphi_{P}\right)$ into equation (5.17) gives an equation which is easily solved numerically:

$$
\begin{aligned}
\partial_{t} h_{q, Z}^{\varphi_{P}}+\beta^{+} \xi^{+} q-\beta^{-} \xi^{-} q & \\
+\sum_{ \pm} \sup _{\gamma^{ \pm} \in\{0,1\}}\left\{\lambda^{ \pm}(Z)\left(\gamma^{ \pm} \frac{\Delta}{2}-\left(\gamma^{ \pm} \mp q\right) \epsilon_{Z}^{ \pm}+h_{q \mp \gamma^{ \pm}, Z}^{\varphi_{P}}-h_{q, Z}^{\varphi_{P}}\right)\right\} & \\
-\frac{\sum_{k \neq Z} G_{Z, k}}{\varphi_{P}} \log \left(\frac{\sum_{k \neq Z} G_{Z, k} e^{-\varphi_{P}\left(h_{q, s}^{\varphi_{P}}-h_{q, Z}^{\varphi_{P}}\right)}}{\sum_{k \neq Z} G_{Z, k}}\right) & =0, \\
h_{q, Z}^{\varphi_{P}}(T) & =-q \ell(q),
\end{aligned}
$$

\subsubsection{Penalties due to ambiguity aversion on regime switching}

By inspecting equations (4.3), (5.14), (5.16), and (5.18) and comparing the terms which contribute to a mixing over different imbalance states, one may notice that each of these terms represents a weighted average of the change in value due to a regime switch, but the averaging methods differ depending on which type of ambiguity is being considered. The four different mixing terms are repeated below for convenience, where $\Delta_{Z, k}=h_{q, k}-h_{q, Z}$. 


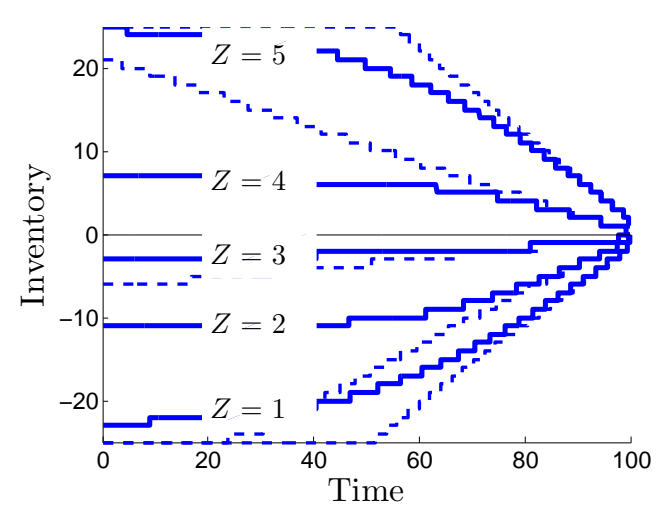

(a) Sell Boundary

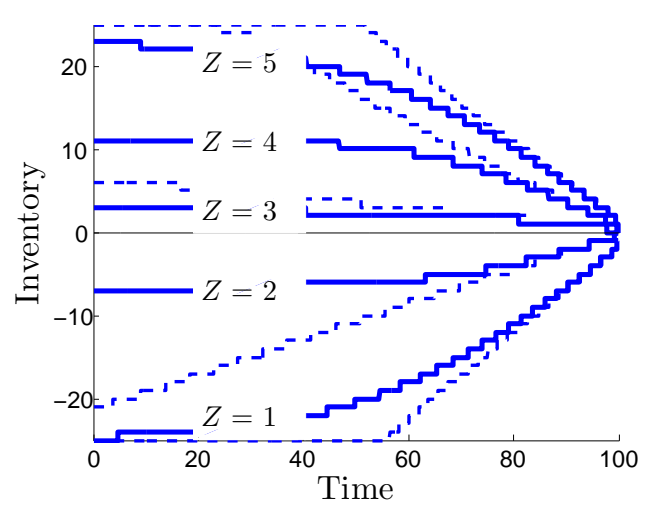

(b) Buy Boundary

Figure 5.5: Trade boundaries for a market maker that is ambiguity averse to transition probabilities of imbalance regimes (dashed lines are ambiguity neutral boundaries). Additional parameters are $\varphi_{R}=0.2, T=100, \ell(q)=-\theta q, \theta=0.005, \bar{q}=-\underline{q}=25$.

\section{i) Ambiguity neutral}

$$
\sum_{k \neq Z} G_{Z, k} \Delta_{Z, k}
$$

ii) Full ambiguity on regime switching

$$
\frac{1}{\varphi} \sum_{k \neq Z} G_{Z, k}\left(1-e^{-\varphi \Delta_{Z, k}}\right)
$$

iii) Ambiguity on rate of switching

$$
\frac{\sum_{k \neq Z} G_{Z, k}}{\varphi}\left(1-\exp \left\{-\varphi \frac{\sum_{k \neq Z} G_{Z, k} \Delta_{Z, k}}{\sum_{k \neq Z} G_{Z, k}}\right\}\right)
$$

iv) Ambiguity on transition probabilities

$$
-\frac{\sum_{k \neq Z} G_{Z, k}}{\varphi} \log \left(\frac{\sum_{k \neq Z} G_{Z, k} e^{-\varphi \Delta_{Z, k}}}{\sum_{k \neq Z} G_{Z, k}}\right)
$$

Some simple analysis shows that term i) is greater than the other three. To show i) is greater than ii), simply note that $\Delta_{Z, k} \geq \frac{1}{\varphi}\left(1-e^{-\varphi \Delta_{Z, k}}\right)$. Similarly, i) is greater than iii) 
because $x \geq \frac{1}{\varphi^{\prime}}\left(1-e^{-\varphi^{\prime} x}\right)$, so letting $x=\sum_{k \neq Z} G_{Z, k} \Delta_{Z, k}$ and $\varphi^{\prime}=\frac{\varphi}{\sum_{k \neq Z} G_{Z, k}}$ yields the result. Finally, to see that i) is greater than iv):

$$
\begin{aligned}
\frac{\sum_{k \neq Z} G_{Z, k} e^{-\varphi \Delta_{Z, k}}}{\sum_{k \neq Z} G_{Z, k}} & \geq \exp \left\{-\varphi \frac{\sum_{k \neq Z} G_{Z, k} \Delta_{Z, k}}{\sum_{k \neq Z} G_{Z, k}}\right\} \\
\log \left(\frac{\sum_{k \neq Z} G_{Z, k} e^{-\varphi \Delta_{Z, k}}}{\sum_{k \neq Z} G_{Z, k}}\right) & \geq-\varphi \frac{\sum_{k \neq Z} G_{Z, k} \Delta_{Z, k}}{\sum_{k \neq Z} G_{Z, k}} \\
-\frac{\sum_{k \neq Z} G_{Z, k}}{\varphi} \log \left(\frac{\sum_{k \neq Z} G_{Z, k} e^{-\varphi \Delta_{Z, k}}}{\sum_{k \neq Z} G_{Z, k}}\right) & \leq \sum_{k \neq Z} G_{Z, k} \Delta_{Z, k}
\end{aligned}
$$

where the first line is a result of Jensen's inequality. Each of these inequalities lead to the conclusion that each type of ambiguity on the imbalance process acts as a penalty when compared to no ambiguity. This is the result seen in Figures 5.3, 5.4, and 5.5. Each ambiguity averse strategy will generally carry less inventory than the ambiguity neutral strategy.

There is one final comparison between these penalties which can be made: Jensen's inequality again implies that term iii) is greater than term ii), meaning that full ambiguity on the regime switching process imposes a larger penalty than ambiguity only on the rate of regime switching. With this comparison in mind, it would be wise to confirm that the market maker will generally hold less inventory in case ii) when compared to case iii). This is indeed shown in Figure 5.6. The dashed lines represent the market maker's trade boundaries when they are ambiguity averse to the full process. The solid lines represent the trade boundaries when only ambiguity aversion is considered on the rate of switches. All of the dashed lines lie closer to zero, indicating a tendency for inventory to be closer to zero in case ii).

Note: only the form of these feedback mixing terms is considered for these inequalities, and it is assumed that the same values for $\Delta_{Z, k}$ are used to derive them. In reality, each type of ambiguity will result in different quantitative values of $\Delta_{Z, k}$ and so these inequalities may not hold in general. This discussion is only meant to indicate that some types of ambiguity generally have stronger or weaker implications on the trading strategy in a feedback sense. 


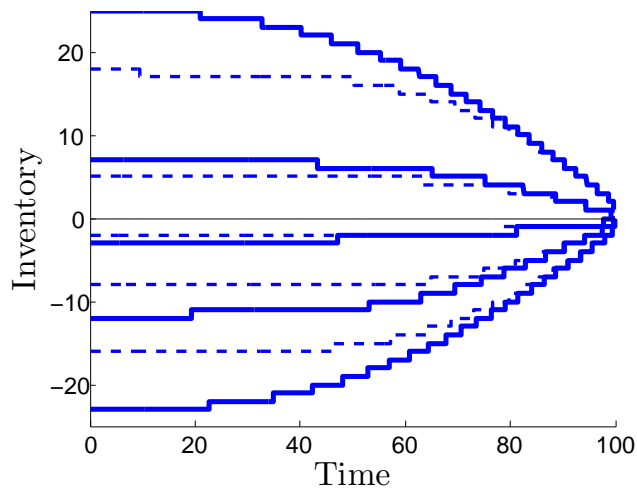

(a) Sell Boundary

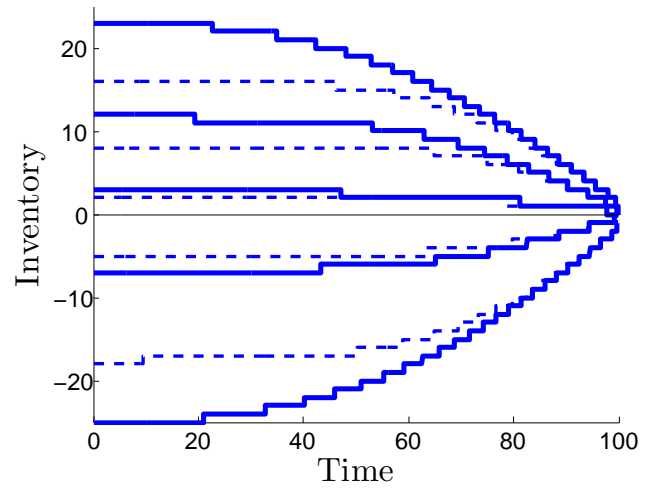

(b) Buy Boundary

Figure 5.6: Trade boundaries for a market maker that is ambiguity averse to imbalance regime process (dashed lines) and ambiguity averse to only rate of regime switches (solid lines). Additional parameters are $\varphi_{G}=0.2, \varphi_{R}=0.2, T=100, \ell(q)=-\theta q, \theta=0.005$, $\bar{q}=-\underline{q}=25$.

\subsection{Ambiguity aversion with respect to rate of mid- price jumps}

In Sections 2.2.3 and 3.2.3, it was shown that ambiguity aversion with respect to the drift of the midprice is equivalent to imposing a quadratic inventory penalization over time. This form of penalty relied on the fact that the midprice process in that case was an arithmetic Brownian motion. In the present setting, the midprice is a pure jump process, so it is unclear what the appropriate equivalent inventory penalization is when compared to ambiguity on the midprice dynamics. This will be answered by considering ambiguity aversion with respect to the rate of midprice changes due to limit order placements and cancellations. That is, the measure changes will be chosen so that the rates of the processes $J_{t}$ are no longer constant at $\beta^{ \pm}$.

To this end, define a set of equivalent measures through the Radon-Nikodym derivatives:

$$
\frac{d \mathbb{Q}(g)}{d \mathbb{P}}=\prod_{ \pm} \exp \left\{-\int_{0}^{T}\left(e^{g_{t}^{ \pm}}-1\right) \beta^{ \pm} d t+\int_{0}^{T} g_{t}^{ \pm} d J_{t}^{ \pm}\right\}
$$

The full class of candidate measures is selected to be:

$$
\mathcal{Q}^{\beta}=\left\{\mathbb{Q}(g): g^{ \pm} \text {are } \mathcal{F}_{t} \text {-predictable, Markov, and bounded }\right\} .
$$


In the measure $\mathcal{Q}(g)$, the processes $J_{t}^{ \pm}$become double stochastic Poisson processes with intensities $e^{g_{t}^{ \pm}} \beta^{ \pm}$. The entropic penalization in this case satisfies:

$$
\mathbb{E}_{t, x, q, S, Z}^{\mathbb{Q}(g)}\left[\mathcal{H}_{t, T}^{\varphi_{\beta}}[\mathbb{Q}(g) \mid \mathbb{P}]\right]=\frac{1}{\varphi_{\beta}} \mathbb{E}_{t, x, q, S, Z}^{\mathbb{Q}(g)}\left[\sum_{ \pm} \int_{t}^{T}\left(e^{g_{u}^{ \pm}}\left(g_{u}^{ \pm}-1\right)+1\right) \beta^{ \pm} d u\right]
$$

and the corresponding infinitesimal generator $\mathcal{L}^{\mathbb{Q}(g)}$ is given by:

$$
\begin{aligned}
\mathcal{L}^{\mathbb{Q}(g)} H^{\beta}\left(t, x, q, S, Z ; \varphi_{\beta}\right) & =e^{g_{t}^{+}} \beta^{+} \mathcal{D}_{L}^{+} H^{\beta}+e^{g_{t}^{-}} \beta^{-} \mathcal{D}_{L}^{-} H^{\beta} \\
+\lambda^{+}(Z) \int_{-\infty}^{\infty} \mathcal{D}_{M}^{+} & H^{\beta} F_{Z}^{+}(y) d y \mathbb{1}_{q \neq \underline{q}}+\lambda^{-}(Z) \int_{-\infty}^{\infty} \mathcal{D}_{M}^{-} H^{\beta} F_{Z}^{-}(y) d y \mathbb{1}_{q \neq \bar{q}} \\
& \quad+\sum_{k=1}^{n} G_{Z, k}\left(H^{\beta}\left(t, x, q, S, k ; \varphi_{\beta}\right)-H^{\beta}\left(t, x, q, S, Z ; \varphi_{\beta}\right)\right) .
\end{aligned}
$$

Making the standard ansatz, $H^{\beta}\left(t, x, q, S, Z ; \varphi_{\beta}\right)=x+q S+h_{q, Z}^{\varphi_{\beta}}(t)$ and substituting into equation (5.2) gives:

$$
\begin{aligned}
\partial_{t} h_{q, Z}^{\varphi_{\beta}}+\inf _{g^{ \pm}}\left\{e^{g^{+}} \beta^{+} \xi^{+} q-e^{g^{-}} \beta^{-} \xi^{-} q+\frac{1}{\varphi_{\beta}} \sum_{ \pm}\left(e^{g^{ \pm}}\left(g^{ \pm}-1\right)+1\right) \beta^{ \pm}\right\} & \\
+\sum_{ \pm} \sup _{\gamma^{ \pm} \in\{0,1\}}\left\{\lambda^{ \pm}(Z)\left(\gamma^{ \pm} \frac{\Delta}{2}-\left(\gamma^{ \pm} \mp q\right) \epsilon_{Z}^{ \pm}+h_{q \mp \gamma^{ \pm}, Z}^{\varphi_{\beta}}-h_{q, Z}^{\varphi_{\beta}}\right)\right\} & \\
+\sum_{k=1}^{n} G_{Z, k}\left(h_{q, k}^{\varphi_{\beta}}-h_{q, Z}^{\varphi_{\beta}}\right) & =0, \quad(5.19) \\
h_{q, Z}^{\varphi_{\beta}}(T) & =-q \ell(q),
\end{aligned}
$$

Proposition 28 (Optimal Feedback Controls). The optimal feedback controls of equation (5.17) are given by:

$$
\begin{aligned}
& \gamma^{+*}\left(t, q, Z ; \varphi_{\beta}\right)=\left\{\begin{array}{lll}
1, & \frac{\Delta}{2}-\epsilon_{Z}^{+}+h_{q-1, Z}^{\varphi_{\beta}}(t)-h_{q, Z}^{\varphi_{\beta}}(t)>0 \quad \text { and } q \neq \underline{q} \\
0, & \frac{\Delta}{2}-\epsilon_{Z}^{+}+h_{q-1, Z}^{\varphi_{\beta}}(t)-h_{q, Z}^{\varphi_{\beta}}(t) \leq 0 \quad \text { or } q=\underline{q}
\end{array},\right. \\
& \gamma^{-*}\left(t, q, Z ; \varphi_{\beta}\right)=\left\{\begin{array}{ll}
1, & \frac{\Delta}{2}-\epsilon_{Z}^{-}+h_{q+1, Z}^{\varphi_{\beta}}(t)-h_{q, Z}^{\varphi_{\beta}}(t)>0 \quad \text { and } q \neq \bar{q} \\
0, & \frac{\Delta}{2}-\epsilon_{Z}^{-}+h_{q+1, Z}^{\varphi_{\beta}}(t)-h_{q, Z}^{\varphi_{\beta}}(t) \leq 0 \quad \text { or } q=\bar{q}
\end{array},\right. \\
& g^{ \pm *}\left(t, q, Z ; \varphi_{\beta}\right)=\mp \varphi_{\beta} \xi^{ \pm} q
\end{aligned}
$$

Proof. The optimization over $\gamma^{ \pm}$is independent from the optimization over $g^{ \pm}$, and equivalent to the ambiguity neutral case. For the optimization over $g^{ \pm}$, first order conditions 
give:

$$
\begin{aligned}
0 & = \pm e^{g^{ \pm}} \beta^{ \pm} \xi^{ \pm} q+\frac{\beta^{ \pm}}{\varphi_{\beta}} e^{g^{ \pm}} g^{ \pm} \\
0 & = \pm \xi^{ \pm} q+\frac{g^{ \pm}}{\varphi_{\beta}} \\
g^{ \pm} & =\mp \varphi_{\beta} \xi^{ \pm} q
\end{aligned}
$$

Checking that this does indeed yield a minimum is easily done by taking a second derivative.

\section{Discussion and financial interpretation}

Substituting the expression for $g^{ \pm *}$ into equation (5.19) gives an equation to be solved numerically:

$$
\begin{aligned}
& \partial_{t} h_{q, Z}^{\varphi_{\beta}}+\frac{\beta^{+}}{\varphi_{\beta}}\left(1-e^{-\varphi_{\beta} \xi^{+} q}\right)+\frac{\beta^{-}}{\varphi_{\beta}}\left(1-e^{\varphi_{\beta} \xi^{-} q}\right) \\
&+\sum_{ \pm} \sup _{\gamma^{ \pm} \in\{0,1\}}\left\{\lambda^{ \pm}(Z)\left(\gamma^{ \pm} \frac{\Delta}{2}-\left(\gamma^{ \pm} \mp q\right) \epsilon_{Z}^{ \pm}+h_{q \mp \gamma^{ \pm}, Z}^{\varphi_{\beta}}-h_{q, Z}^{\varphi_{\beta}}\right)\right\} \\
&+\sum_{k=1}^{n} G_{Z, k}\left(h_{q, k}^{\varphi_{\beta}}-h_{q, Z}^{\varphi_{\beta}}\right)=0, \\
& h_{q, Z}^{\varphi_{\beta}}(T)=-q \ell(q),
\end{aligned}
$$

In equation (5.20), terms of the form $\frac{\beta^{+}}{\varphi_{\beta}}\left(1-e^{-\varphi_{\beta} \xi^{+} q}\right)$ and $\frac{\beta^{-}}{\varphi_{\beta}}\left(1-e^{\varphi_{\beta} \xi^{-} q}\right)$ appear when compared to the original equation (4.3). This is analogous to the appearance of the term $-\frac{1}{2} \varphi_{\mu} \sigma^{2} q^{2}$ in equation (2.15) when compared to equation (2.4). In Section 2.2.3, the term $-\frac{1}{2} \varphi_{\mu} \sigma^{2} q^{2}$ was shown to be equivalent to the introduction of a quadratic inventory penalization with corresponding value function and optimization problem in equation (2.16). The sum of the two exponentials above gives a term which is equal to 0 at $q=0$ and which is concave in $q$, similar conditions shared by $-\frac{1}{2} \varphi_{\mu} \sigma^{2} q^{2}$, and conditions that should be satisfied by any reasonable inventory penalization. This can be interpreted as solving an ambiguity neutral market making problem, but with an inventory penalization of the following form: 


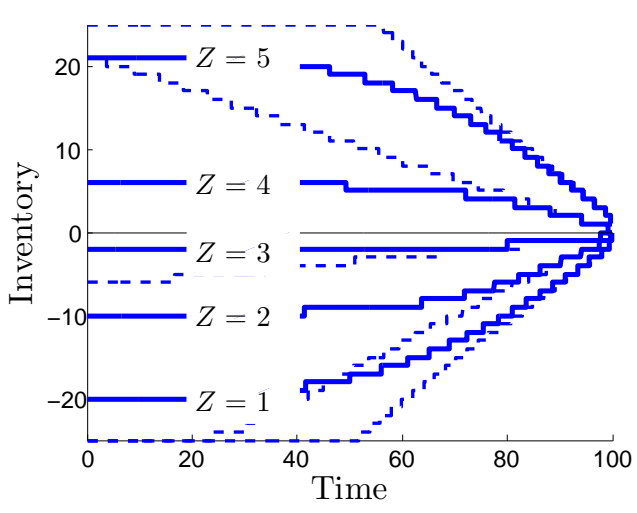

(a) Sell Boundary

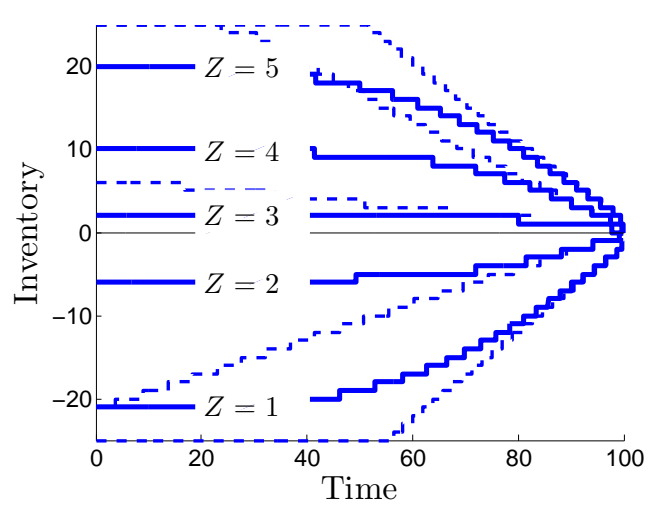

(b) Buy Boundary

Figure 5.7: Trade boundaries for a market maker that is ambiguity averse to rate of midprice jumps (dashed lines are ambiguity neutral boundaries). Additional parameters are $\varphi_{\beta}=0.5, T=100, \ell(q)=-\theta q, \theta=0.005, \bar{q}=-\underline{q}=25$.

$H^{\phi}(t, x, q, S, Z)=\sup _{\left(\gamma_{u}^{ \pm}\right)_{t \leq u \leq T} \in \mathcal{A}} \mathbb{E}_{t, x, q, S, Z}^{\mathbb{P}}\left[X_{T}+q_{T}\left(S_{T}-\ell\left(q_{T}\right)\right)-\frac{1}{\phi} \sum_{ \pm} \int_{t}^{T} \beta^{ \pm}\left(1-e^{\mp \phi \xi^{ \pm} q_{u}}\right) d u\right]$

In Figure 5.8, the strengths of these two penalizations is compared. The parameters of the quadratic penalty are chosen so that both penalties are equal when $q= \pm 1$. It is clear for that this set of parameters, the magnitude of the penalties is nearly identical for values of $q$ near 0 (they are only exactly equal at $q=0$ and $q= \pm 1$ ). Of course, as $q$ gets very large, the exponential penalty will become stronger. 


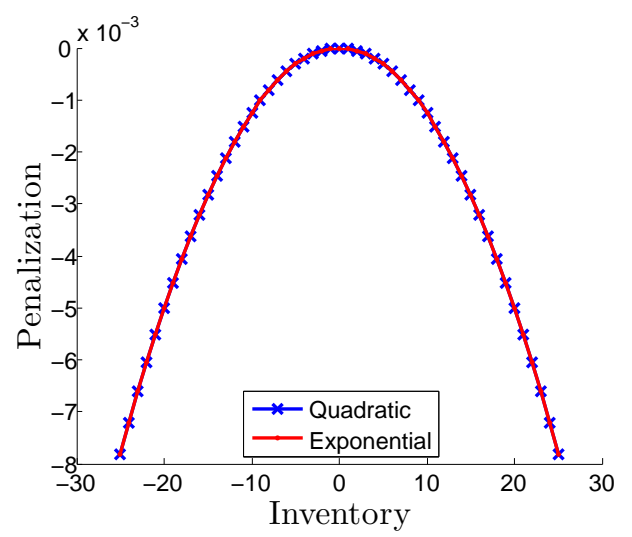

(a) $\varphi_{\beta}=0.5, \xi^{ \pm}=0.005$

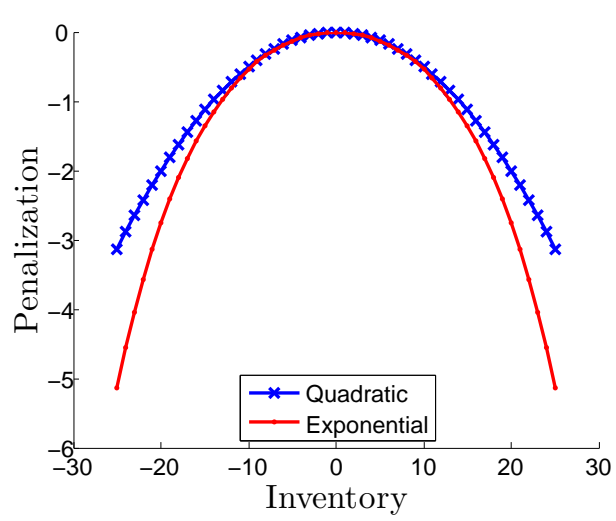

(b) $\varphi_{\beta}=2, \xi^{ \pm}=0.05$

Figure 5.8: Magnitude of inventory penalizations for quadratic and exponential penalties. Additional parameters are $\beta^{ \pm}=1$. 


\section{Chapter 6}

\section{Future Work}

\subsection{Model Uncertainty and Ambiguity Aversion}

The effects of model uncertainty in finance is an ongoing area of study within many areas beyond high frequency and algorithmic trading. Of particular interest and importance are cases when behaviour due to ambiguity aversion is qualitatively different than behaviour due to risk aversion. The existence of such cases has already been demonstrated in Sections 2.3 and 3.2.4 where it was seen that different types of ambiguity lead to different changes in optimal trading strategies. There is no uniform change that can be made to the trading strategy which corresponds to all types of ambiguity, and so this ambiguity averse behaviour can not be equivalent in all cases to risk averse behaviour. The discovery of additional cases where this distinction occurs is important because it means that standard risk averse agents are still exposed to different types of risks due to model uncertainty if it is not explicitly considered.

Within the specific context of high frequency and algorithmic trading, the effects of ambiguity aversion have yet to be investigated when an agent considers multiple assets or when the market has different priority rules for matching orders. In the case of multiple assets, it is possible that complex structure between assets can give rise to unforeseen modelling risks (for example, consider foreign exchange trading, in which the rates between three currencies should tend to satisfy certain relationships). In the case of different priority rules, vastly different market structures could give rise to different changes in trading strategies. In this work, strict priority rules were not generally adhered 
to, but the model assumptions dictating how limit orders are filled by market orders closely resembles a chronological priority. Incorporating different priority rules, such as a pro rata structure, and performing the appropriate ambiguity aversion analysis should be performed.

Finally, the interpretation of certain ambiguity aversion results as giving rise to commonly used risk controls (as in Sections 2.2.3 and 3.2.3 for the cumulative inventory penalization, and Proposition 24 for minimum and maximum inventory constraints) leads to the question of whether there are others with similar interpretations. Further, it also gives motivation for how to properly incorporate similar risk controls when the model does not easily lend itself to techniques that have been used in the past (as in Section 5.4). If additional risk control type interpretations of ambiguity aversion results are discovered, then these relationships will give motivation for how to properly specify similar risk controls in more general situations.

\subsection{Limit Order Queues}

In this work, there were two different methods for modelling in the reference measure how market orders were matched with the limit orders placed by the agent. In Chapters 2 and 3, a probability of fill was assigned to any limit order of the agent. This probability only depended on the distance of the limit order price from the midprice. In Chapters 4 and 5, any limit order placed at the best bid or ask would be filled by any market order with probability 1 (this assumption is not difficult to generalize to a probability $p$ with nearly the same analysis and results). The first assumption appears to give too much control to the agent when compared to what happens in practice (as discussed in Section 4.1 ), and the second assumption is an oversimplification.

The most significant difference between what happens in the two models considered here and reality is the existence of a queue for all limit orders at a single price. This queue ensures that limit orders that are submitted first get matched with market orders before limit orders that were submitted later if there is not enough volume to fill all orders at a certain price. In essence, the longer a limit order has been in existence, the larger its probability of being filled. There is still be dependence on the probability of fill with the distance of the limit order from the midprice, but due to the fact that limit orders exist at a fixed price (not at a fixed distance from the midprice), constant cancellation and 
resubmission of a limit order due to fluctuations in the midprice leave little chance of the order ever reaching the front of the queue. By incorporating the resting time of a limit order as an additional state variable, it is possible that a more realistic model for the fill probability can be considered at the cost of tractability (such a model must be supported by data), and appropriate optimization of limit order placement can be investigated.

\subsection{Model Improvements}

As discussed in Section 4.1, the model of Chapters 2 and 3 do not perform well when adapted to real trading environments with discrete tick sizes. The incorporation of the imbalance process as a predictor and fixed tick size show improvements upon the earlier model through minimal amounts of backtesting, but are still not a significant improvement in performance. Backtesting shows that the agent still suffers from price movements, even when they may elect not to trade depending on the current imbalance regime. There are two model assumptions made in Chapter 4 which lend themselves the easiest to immediate attention.

First, in equation (4.1), the changes in the midprice which are not due to the arrival of market orders are assumed to be independent of imbalance. The magnitude of such a change is always almost limited to a half tick (reflected by the constant values of $\xi^{ \pm}$), but the rates of such movements may be affected by the imbalance process. Ignoring these effects exposes the agent to price trends which they assume are not present.

Second, the best bid and ask prices are assumed to be a fixed distance, $\frac{\Delta}{2}$, from the midprice. Although in many equities the spread does spend a large portion of the time at a fixed value, it certainly does change with respect to time. Even with keeping the restriction that the agent may only post at the best bid and ask, modifying the possible values of these prices will more accurately reflect reality and likely improve the agent's performance. Further, there may also be strong predictive power in the spread, or jointly in the spread and imbalance, which is not being accounted for.

A more complicated model adjustment would incorporate behaviour which is completely ignored through this work: the effect of the agent's order placements on the dynamics of the market. In this work, the placement of a limit order has no effect on the dynamics of incoming market orders or the dynamics of the midprice. In reality, since so much 
trading is done by computers, and many of their strategies are sensitive to changes in the state of the limit order book, changes in the agent's strategy must be accounted for in how it affects other factors. 


\section{Appendix A}

\section{Background Material}

\section{A.1 The Hamilton-Jacobi-Bellman PDE}

In Section 2.1, an informal justification for the validity of the HJB equation was given. This argument implicitly made some assumptions which can be eliminated with a more detailed argument, most importantly the assumption that an optimal control exists. This section will not be in the context of algorithmic trading, but will consider a general stochastic control problem.

For any choice of $u_{t}=u\left(t, X_{t}\right)$, where $u:[0, T] \times \mathbb{R} \rightarrow U \subset \mathbb{R}$, let:

$$
d X_{t}^{u}=\alpha\left(t, X_{t}^{u}, u_{t}\right) d t+\sigma\left(t, X_{t}^{u}, u_{t}\right) d W_{t}+\int_{-\infty}^{\infty} f\left(t, X_{t^{-}}^{u}, u_{t^{-}}, y\right) \mu(d y, d t)
$$

where all coefficients have sufficient growth conditions so that $X_{t}^{u}$ is well defined, and $\mu$ has compensator $\nu_{t}(d y, d t)=\lambda_{X_{t}} F_{X_{t}}(y) d y d t$. The infinitesimal generator of the process $X_{T}^{u}$ acts as follows:

$$
\begin{aligned}
\mathcal{L}^{u} \Phi(t, x)=\alpha(t, x, u(t, x)) \partial_{x} & \Phi(t, x)+\frac{1}{2} \sigma^{2}(t, x, u(t, x)) \partial_{x x} \Phi(t, x) \\
& +\lambda_{x} \int_{-\infty}^{\infty}(\Phi(t, x+f(t, x, u(t, x), y))-\Phi(t, x)) F_{x}(y) d y
\end{aligned}
$$

Let $J^{u}(t, x)$ be the performance criteria of the control $u$ when the process $X^{u}$ is started 
at time $t$ with initial data $x$ so that:

$$
J^{u}(t, x)=\mathbb{E}_{t, x}\left[\int_{t}^{T} h\left(s, X_{s}^{u}, u\left(s, X_{s}\right)\right) d s+\Psi\left(X_{T}^{u}\right)\right]
$$

The goal of the optimization is to maximize $J^{u}(t, x)$ over the set of all Markov controls $u \in \mathcal{U}$, where

$$
\mathcal{U}=\{u:[0, T] \times \mathbb{R} \rightarrow U \subset \mathbb{R}\}
$$

Define the value function to be the supremum of the performance criteria:

$$
H(t, x)=\sup _{u \in \mathcal{U}} J^{u}(t, x)=\sup _{u \in \mathcal{U}} \mathbb{E}_{t, x}\left[\int_{t}^{T} h\left(s, X_{s}^{u}, u\left(s, X_{s}\right)\right) d s+\Psi\left(X_{T}^{u}\right)\right]
$$

The following assumptions are made:

- The value function $H(t, x)$ satisfies the Dynamic Programming Principle:

$$
H(t, x)=\sup _{u \in \mathcal{U}} \mathbb{E}_{t, x}\left[\int_{t}^{\tau} h\left(s, X_{s}^{u}, u\left(s, X_{s}^{u}\right)\right) d s+H\left(\tau, X_{\tau}^{u}\right)\right]
$$

for any stopping time $\tau$ such that $t \leq \tau \leq T$.

- $H \in C^{1,2}([0, T] \times \mathbb{R})$.

It will be shown that with these assumptions the value function $H$ satisfies the HamiltonJacobi-Bellman (HJB) equation:

$$
\begin{aligned}
\partial_{t} H(t, x)+\sup _{u \in U}\left(\mathcal{L}^{u} H(t, x)+h(t, x, u)\right) & =0 \\
H(T, x) & =\Psi(x)
\end{aligned}
$$

The terminal conditions are clear from the definition of the performance criteria. From 
the Dynamic Programming Principle, the value function satisfies:

$$
\begin{aligned}
H(t, x) \geq & \mathbb{E}_{t, x}\left[\int_{t}^{\tau} h\left(s, X_{s}^{u}, u\left(s, X_{s}^{U}\right)\right) d s+H\left(\tau, X_{\tau}^{u}\right)\right] \\
0 \geq & \mathbb{E}_{t, x}\left[\int_{t}^{\tau} h\left(s, X_{s}^{u}, u\left(s, X_{s}^{u}\right)\right) d s+H\left(\tau, X_{\tau}^{u}\right)-H(t, x)\right] \\
= & \mathbb{E}_{t, x}\left[\int_{t}^{\tau} h\left(s, X_{s}^{u}, u\left(s, X_{s}^{u}\right)\right) d s\right. \\
& +\int_{t}^{\tau}\left(\partial_{t}+\alpha\left(s, X_{s}^{u}, u\left(s, X_{s}^{u}\right)\right) \partial_{x}+\frac{1}{2} \sigma^{2}\left(s, X_{s}^{u}, u\left(s, X_{s}^{u}\right)\right) \partial_{x x}\right) H\left(s, X_{s}^{u}\right) d s \\
& +\int_{t}^{\tau} \sigma\left(s, X_{s}^{u}, u\left(s, X_{s}^{u}\right)\right) \partial_{x} H\left(s, X_{s}^{u}\right) d W_{s} \\
& \left.+\int_{t}^{\tau} \int_{-\infty}^{\infty}\left(H\left(s, X_{s^{-}}^{u}+f\left(s, X_{s^{-}}^{u}, u\left(s, X_{s^{-}}^{u}\right), y\right)\right)-H\left(s, X_{s^{-}}^{u}\right)\right) \mu(d y, d s)\right]
\end{aligned}
$$

By selecting $\tau=\inf \left\{s>t: X_{s}>R\right\} \wedge t^{\prime}$ where $t<t^{\prime} \leq T$, this expectation becomes:

$$
\begin{aligned}
0 \geq & \mathbb{E}_{t, x}\left[\int_{t}^{\tau} h\left(s, X_{s}^{u}, u\left(s, X_{s}^{u}\right)\right) d s\right. \\
& +\int_{t}^{\tau}\left(\partial_{t}+\alpha\left(s, X_{s}^{u}, u\left(s, X_{s}^{u}\right)\right) \partial_{x}+\frac{1}{2} \sigma^{2}\left(s, X_{s}^{u}, u\left(s, X_{s}^{u}\right)\right) \partial_{x x}\right) H\left(s, X_{s}^{u}\right) d s \\
& \left.+\int_{t}^{\tau} \int_{-\infty}^{\infty} \lambda_{X_{s}^{u}}\left(H\left(s, X_{s^{-}}^{u}+f\left(s, X_{s^{-}}^{u}, u\left(s, X_{s^{-}}^{u}\right), y\right)\right)-H\left(s, X_{s^{-}}^{u}\right)\right) F_{X_{s^{-}}^{u}}(y) d y d s\right] \\
= & \mathbb{E}_{t, x}\left[\int_{t}^{\tau} h\left(s, X_{s}^{u}, u\left(s, X_{s}^{u}\right)\right) d s+\int_{t}^{\tau}\left(\partial_{t}+\mathcal{L}^{u}\right) H\left(s, X_{s}^{u}\right) d s\right]
\end{aligned}
$$

Dividing by $\mathbb{E}_{t, x}[\tau-t]$ and letting $t^{\prime} \rightarrow t$, the above becomes:

$$
\begin{aligned}
& 0 \geq\left(\partial_{t}+\mathcal{L}^{u}\right) H(t, x)+h(t, x, u(t, x)) \\
& 0 \geq \partial_{t} H(t, x)+\sup _{u \in U}\left(\mathcal{L}^{u} H(t, x)+h(t, x, u)\right)
\end{aligned}
$$

To show the reverse inequality, choose the same stopping time as above, let $\epsilon>0$ and choose $u^{\epsilon} \in \mathcal{U}$ so that:

$$
H(t, x)-\epsilon\left(t^{\prime}-t\right) \leq \mathbb{E}_{t, x}\left[\int_{t}^{\tau} h\left(s, X_{s}^{u^{\epsilon}}, u^{\epsilon}\left(s, X_{s}^{u^{\epsilon}}\right)\right) d s+H\left(\tau, X_{\tau}^{u^{\epsilon}}\right)\right]
$$




$$
\begin{aligned}
-\epsilon\left(t^{\prime}-t\right) \leq & \mathbb{E}_{t, x}\left[\int_{t}^{\tau} h\left(s, X_{s}^{u^{\epsilon}}, u^{\epsilon}\left(s, X_{s}^{u^{\epsilon}}\right)\right) d s+H\left(\tau, X_{\tau}^{u^{\epsilon}}\right)-H(t, x)\right] \\
-\epsilon\left(t^{\prime}-t\right) \leq & \mathbb{E}_{t, x}\left[\int_{t}^{\tau} h\left(s, X_{s}^{u^{\epsilon}}, u^{\epsilon}\left(s, X_{s}^{u^{\epsilon}}\right)\right) d s\right. \\
& +\int_{t}^{\tau}\left(\partial_{t}+\alpha\left(s, X_{s}^{u^{\epsilon}}, u^{\epsilon}\left(s, X_{s}^{u^{\epsilon}}\right)\right) \partial_{x}+\frac{1}{2} \sigma^{2}\left(s, X_{s}^{u^{\epsilon}}, u^{\epsilon}\left(s, X_{s}^{u^{\epsilon}}\right)\right) \partial_{x x}\right) H\left(s, X_{s}^{u^{\epsilon}}\right) d s \\
& +\int_{t}^{\tau} \sigma\left(s, X_{s}^{u^{\epsilon}}, u^{\epsilon}\left(s, X_{s}^{u^{\epsilon}}\right)\right) \partial_{x} H\left(s, X_{s}^{u^{\epsilon}}\right) d W_{s} \\
& \left.+\int_{t}^{\tau} \int_{-\infty}^{\infty}\left(H\left(s, X_{s^{-}}^{u^{\epsilon}}+f\left(s, X_{s^{-}}^{u^{\epsilon}}, u^{\epsilon}\left(s, X_{s^{-}}^{u^{\epsilon}}\right), y\right)\right)-H\left(s, X_{s^{-}}^{u^{\epsilon}}\right)\right) \mu(d y, d s)\right] \\
-\epsilon\left(t^{\prime}-t\right) \leq & \mathbb{E}_{t, x}\left[\int_{t}^{\tau} h\left(s, X_{s}^{u^{\epsilon}}, u^{\epsilon}\left(s, X_{s}^{u^{\epsilon}}\right)\right) d s\right. \\
& +\int_{t}^{\tau}\left(\partial_{t}+\alpha\left(s, X_{s}^{u^{\epsilon}}, u^{\epsilon}\left(s, X_{s}^{u^{\epsilon}}\right)\right) \partial_{x}+\frac{1}{2} \sigma^{2}\left(s, X_{s}^{u^{\epsilon}}, u^{\epsilon}\left(s, X_{s}^{u^{\epsilon}}\right)\right) \partial_{x x}\right) H\left(s, X_{s}^{u^{\epsilon}}\right) d s \\
& \left.+\int_{t}^{\tau} \int_{-\infty}^{\infty} \lambda_{X_{s}^{u^{\epsilon}}}\left(H\left(s, X_{s^{-}}^{u^{\epsilon}}+f\left(s, X_{s^{-}}^{u^{\epsilon}}, u^{\epsilon}\left(s, X_{s^{-}}^{u^{\epsilon}}\right), y\right)\right)-H\left(s, X_{s^{-}}^{u^{\epsilon}}\right)\right) F_{X_{s^{-}}}(y) d y d s\right] \\
-\epsilon\left(t^{\prime}-t\right) \leq & \mathbb{E}_{t, x}\left[\int_{t}^{\tau}\left(\partial_{t}+\mathcal{L}^{u^{\epsilon}}+h\left(s, X_{s}^{u^{\epsilon}}, u^{\epsilon}\left(s, X_{s}^{u^{\epsilon}}\right)\right)\right) H\left(s, X_{s}^{u^{\epsilon}}\right) d s\right] \\
-\epsilon\left(t^{\prime}-t\right) \leq & \mathbb{E}_{t, x}\left[\int_{t}^{\tau} \sup _{u \in U}\left(\partial_{t}+\mathcal{L}^{u}+h\left(s, X_{s}^{u}, u\left(s, X_{s}^{u}\right)\right)\right) H\left(s, X_{s}^{u}\right) d s\right]
\end{aligned}
$$

Dividing again by $\mathbb{E}_{t, x}[\tau-t]$ and taking $t^{\prime} \rightarrow t$ gives:

$$
-\epsilon \leq \partial_{t} H(t, x)+\sup _{u \in U}\left(\mathcal{L}^{u} H(t, x)+h(t, x, u)\right)
$$

Since this is true for all $\epsilon>0$, combining (A.1) and (A.2) give the HJB PDE. For more details, see Yong and Zhou (1999), Fleming and Soner (2006), Pham (2009), and Øksendal and Sulem (2009).

\section{A.2 Poisson Random Measures}

This work makes use in several locations of Poisson random measures for modelling purposes as they are particularly useful for modelling processes with jumps. This section will give the definition of a Poisson random measure followed by an equivalent interpretation which helps with its conceptual use. Finally, a brief discussion on Girsanov's Theorem for Poisson random measures will be presented so that computations of relative entropy can be performed when a measure change is generated through the use of a Poisson random 
measure.

Let $\left(\mathbb{R}^{+} \times E, \mathcal{B}\left(\mathbb{R}^{+} \times E\right), \nu\right)$ be a measure space with $\sigma$-finite measure $\nu(d t, d y)$, and let $(\Omega, \mathcal{F}, \mathbb{P})$ be a probability space. Then $\mu$ is a Poisson random measure on $(\Omega, \mathcal{F}, \mathbb{P})$ with compensator $\nu$ if:

- For all $A \in \mathcal{B}\left(\mathbb{R}^{+} \times E\right), \mu(A)$ is a Poisson random variable with parameter $\nu(A)$.

- If $A_{1}, \ldots, A_{n}$ are disjoint, then $\mu\left(A_{i}\right)$ are mutually independent.

- For all $\omega \in \Omega, \mu_{\omega}(\cdot)$ is a measure on $\left(\mathbb{R}^{+} \times E, \mathcal{B}\left(\mathbb{R}^{+} \times E\right)\right)$.

In this work, the set $E$ is either equal to $\mathbb{R}$ when the Poisson random measure is used to model the arrival of market orders, or a finite state space $\mathcal{Z}$ when it is used to model the evolution of a continuous time Markov chain. If $\nu$ is a finite measure on $\left(\mathbb{R}^{+} \times E, \mathcal{B}\left(\mathbb{R}^{+} \times\right.\right.$ $E)$ ) (as it is in each case of this work), then $\mu$ is equivalently defined as follows: Let $X_{k}$ be i.i.d. with distribution $\frac{\nu}{\nu\left(\mathbb{R}^{+} \times E\right)}$, and let $Y$ be a Poisson random variable with parameter $\nu\left(\mathbb{R}^{+} \times E\right)$. Then:

$$
\mu_{\omega}(\cdot)=\sum_{n=1}^{Y(\omega)} \delta_{X_{i}(\omega)}(\cdot)
$$

where $\delta_{x}$ is the Dirac point mass centred at $x$. In the case of modelling the arrival of market orders with a Poisson random measure, this representation is interpreted as each $\delta_{X_{i}}$ representing a single market order. The $t \in \mathbb{R}^{+}$dimension of $X_{i}$ is the time of the incoming order, and the $y \in E$ dimension is some other quantity related to the interaction of market orders and limit orders (in Chapters 2 and 3,y represents the maximum distance from the midprice $S_{t}$ that was executed by the market order. In Chapters 4 and 5, $y$ represents the change in the midprice immediately following the market order).

\section{A.2.1 Girsanov's Theorem for Poisson random measures}

There are numerous locations in this work where a measure change is defined in terms of a Poisson random measure. When such a measure change is used, it is important to know the dynamics of the involved processes in the new measure. Suppose a process is 
defined by:

$$
\begin{aligned}
\frac{d \eta_{t}}{\eta_{t^{-}}} & =\int_{y \in E}\left(e^{g_{t}(y)}-1\right)(\mu(d t, d y)-\nu(d t, d y)) \\
\eta_{0} & =1
\end{aligned}
$$

If $g$ is chosen so that $\left.\mathbb{E}^{\mathbb{P}}\left[\left|\eta_{t}\right|\right]<\infty\right]$, then $\eta$ is a martingale and can be used to define a measure change process by:

$$
\left(\frac{d \mathbb{Q}}{d \mathbb{P}}\right)_{t}=\eta_{t}
$$

The process $\eta$ can be written in terms of $\mu$ and $\nu$ by noting the following: if there is no jump due to $\mu$ at time $t$, then $\eta$ simply decays exponentially with rate $\int_{y \in E}\left(e^{g_{t}(y)}-\right.$ 1) $\nu(d t, d t)$. At any time $t$ where there is a jump, the process behaves as $\eta_{t} \mapsto \eta_{t}+$ $\eta_{t}\left(e^{g_{t}(y)}-1\right)=\eta_{t} e^{g_{t}(y)}$. This leads to:

$$
\eta_{t}=\exp \left\{-\int_{0}^{t} \int_{y \in E}\left(e^{g_{s}(y)}-1\right) \nu(d s, d y)+\int_{0}^{t} \int_{y \in E} g_{s}(y) \mu(d s, d y)\right\}
$$

The question of interest now is: what is the compensator of $\mu$ ?

Theorem 29 (Girsanov's Theorem for Poisson random measures). Let the measure $\mathbb{Q}$ be defined by using the process in equation (A.3) as a Radon-Nikodym derivative process. Let $\nu^{\mathbb{Q}}$ denote the compensator for $\mu$ in $\mathbb{Q}$. Then $\nu^{\mathbb{Q}}(d t, d y)=e^{g_{t}(y)} \nu(d t, d y)$.

The easiest way to observe the result of this theorem is to realize that any process defined by:

$$
\frac{d \zeta_{t}}{\zeta_{t^{-}}}=\int_{y \in E}\left(e^{h_{t}(y)}-1\right)\left(\mu(d t, d y)-\nu^{\mathbb{Q}}(d t, d y)\right)
$$

must be a $\mathbb{Q}$-martingale for any $h$ that is $\mathcal{F}_{t}$ predictable such that $\mathbb{E}^{\mathbb{Q}}\left[\left|\zeta_{t}\right|\right]<\infty$, and then simply use this to compute $\nu^{\mathbb{Q}}$. For similar arguments used to arrive at equation (A.3), the process $\zeta_{t}$ may be written:

$$
\zeta_{t}=\exp \left\{-\int_{0}^{t} \int_{y \in E}\left(e^{h_{s}(y)}-1\right) \nu^{\mathbb{Q}}(d s, d y)+\int_{0}^{t} \int_{y \in E} h_{s}(y) \mu(d s, d y)\right\}
$$


Now substituting into the martingale condition:

$$
\begin{aligned}
\zeta_{s}= & \mathbb{E}^{\mathbb{Q}}\left[\zeta_{t} \mid \mathcal{F}_{s}\right] \\
\zeta_{s}= & \mathbb{E}^{\mathbb{P}}\left[\zeta_{t} \frac{\eta_{t}}{\eta_{s}} \mid \mathcal{F}_{s}\right] \\
\zeta_{s}= & \zeta_{s} \mathbb{E}^{\mathbb{P}}\left[\frac{\zeta_{t}}{\zeta_{s}} \frac{\eta_{t}}{\eta_{s}} \mid \mathcal{F}_{s}\right] \\
1= & \mathbb{E}^{\mathbb{P}}\left[\exp \left\{-\int_{s}^{t} \int_{y \in E}\left(e^{h_{s}(y)}-1\right) \nu^{\mathbb{Q}}(d s, d y)+\int_{s}^{t} \int_{y \in E} h_{s}(y) \mu(d s, d y)\right\} \times\right. \\
1= & \quad \mathbb{E}^{\mathbb{P}}\left[\exp \left\{-\int_{s}^{t} \int_{y \in E}\left(e^{h_{s}(y)}-1\right) \nu^{\mathbb{Q}}(d s, d y)+\left(e^{g_{s}(y)}-1\right) \nu(d s, d y)+\int_{s}^{t} \int_{y \in E} g_{s}(y) \mu(d s, d y)\right\} \mid \mathcal{F}_{s}\right] \\
& \left.\left.\quad+\int_{s}^{t} \int_{y \in E}\left(h_{s}(y)+g_{s}(y)\right) \mu(d s, d y)\right\} \mid \mathcal{F}_{s}\right]
\end{aligned}
$$

Note that for this equation:

$$
\begin{aligned}
\int_{s}^{t} \int_{y \in E}\left(e^{h_{s}(y)+g_{s}(y)}-1\right) \nu(d s, d y) & =\int_{s}^{t} \int_{y \in E}\left(e^{h_{s}(y)}-1\right) \nu^{\mathbb{Q}}(d s, d y)+\left(e^{g_{s}(y)}-1\right) \nu(d s, d y) \\
0 & =\int_{s}^{t} \int_{y \in E}\left(e^{h_{s}(y)}-1\right) \nu^{\mathbb{Q}}(d s, d y) \\
& +\left(\left(e^{g_{s}(y)}-1\right)-\left(e^{h_{s}(y)+g_{s}(y)}-1\right)\right) \nu(d s, d y) \\
0 & =\int_{s}^{t} \int_{y \in E}\left(e^{h_{s}(y)}-1\right) \nu^{\mathbb{Q}}(d s, d y)-\left(e^{h_{s}(y)}-1\right) e^{g_{s}(y)} \nu(d s, d y) \\
0 & =\int_{s}^{t} \int_{y \in E}\left(e^{h_{s}(y)}-1\right)\left(\nu^{\mathbb{Q}}(d s, d y)-e^{g_{s}(y)} \nu(d s, d y)\right)
\end{aligned}
$$

This is clearly only valid when $\nu^{\mathbb{Q}}(d t, d y)=e^{g_{t}(y)} \nu(d t, d y)^{1}$. The relative entropy between $\mathbb{Q}$ and $\mathbb{P}$ is also of interest. By using this compensator under the measure $\mathbb{Q}$ and

\footnotetext{
${ }^{1}$ For more information regarding measure changes involving Poisson random measures, see Jacod and Shiryaev (1987) Chapter III.3
} 
performing a direct computation, it is seen to satisfy:

$$
\begin{aligned}
\mathbb{E}^{\mathbb{Q}}\left[\log \left(\frac{d \mathbb{Q}}{d \mathbb{P}}\right)_{t}\right] & =\mathbb{E}^{\mathbb{Q}}\left[\log \left(\eta_{t}\right)\right] \\
& =\mathbb{E}^{\mathbb{Q}}\left[-\int_{0}^{t} \int_{y \in E}\left(e^{g_{s}(y)}-1\right) \nu(d s, d y)+\int_{0}^{t} \int_{y \in E} g_{s}(y) \mu(d s, d y)\right] \\
& =\mathbb{E}^{\mathbb{Q}}\left[-\int_{0}^{t} \int_{y \in E}\left(e^{g_{s}(y)}-1\right) \nu(d s, d y)+\int_{0}^{t} \int_{y \in E} g_{s}(y) \nu^{\mathbb{Q}}(d s, d y)\right] \\
& =\mathbb{E}^{\mathbb{Q}}\left[-\int_{0}^{t} \int_{y \in E}\left(e^{g_{s}(y)}-1\right) \nu(d s, d y)+\int_{0}^{t} \int_{y \in E} g_{s}(y) e^{g_{t}(y)} \nu(d s, d y)\right] \\
& =\mathbb{E}^{\mathbb{Q}}\left[\int_{0}^{t} \int_{y \in E}\left(e^{g_{s}(y)}\left(g_{s}(y)-1\right)+1\right) \nu(d s, d y)\right]
\end{aligned}
$$

\section{A.3 The Transition Groupoid}

This section will serve to accomplish two goals. First, the transition groupoid and an action on the state space is defined. This is necessary because the state space of imbalance regimes does not have an additive structure, but there is a large benefit in using Poisson random measures to model processes because of their well understood structure. Second, once an alternate definition of the imbalance regime process is given, it will be shown that this definition does in fact have the same dynamics as the original one.

Let $\mathcal{Z}=\{1, \ldots, n\}$. Then let $\mathcal{T}_{\mathcal{Z}}$ denote the transition groupoid:

$$
\mathcal{T}_{\mathcal{Z}}=\{\langle a, b\rangle: a, b \in \mathcal{Z}\}
$$

equipped with inverse operator $\left.\langle a, b\rangle^{-1}=<b, a\right\rangle$ and partial function + defined by $\langle a, b\rangle+\langle b, c\rangle=\langle a, c\rangle$. The action of $\mathcal{T}_{\mathcal{Z}}$ on $\mathcal{Z}$ is defined by $a+\langle a, b\rangle=b$. Intuitively, $\langle a, b\rangle$ represents a transition event from imbalance regime $a$ to imbalance regime $b$. In Chapter 4 , the imbalance regime process was defined to be a continuous time Markov chain with generator matrix $G$. In equation (5.12), the process was redefined in terms of a Poisson random measure using the transition groupoid $\mathcal{T}_{\mathcal{Z}}$. The claim that these definitions result in the same dynamics is now shown. Let $Z_{t}$ satisfy equation 


$$
d Z_{t}=\int_{\mathcal{Z}}<Z_{t^{-}}, s>\pi(d s, d t)
$$

where the compensator of $\pi$ is $\rho_{t}^{\mathbb{P}}(d s, d t)=\sum_{k \neq Z_{t}} G_{Z_{t}, k} \delta_{k}(s) d s d t$. Let $\Phi: \mathcal{Z} \rightarrow \mathbb{R}$, and define:

$$
F_{t}=\mathbb{E}\left[\Phi\left(Z_{T}\right) \mid \mathcal{F}_{t}\right]
$$

Clearly $F_{t}$ is a martingale and $F_{t}=f\left(t, Z_{t}\right)$ for some function $f:[0, T] \times \mathcal{Z} \rightarrow \mathbb{R}$. Then $f$ satisfies the equation:

$$
\begin{aligned}
0 & =\partial_{t} f(t, Z)+\int_{\mathcal{Z}}(f(t, Z+<Z, s>)-f(t, Z)) \sum_{k \neq Z} G_{Z, k} \delta_{k}(s) d s \\
0 & =\partial_{t} f(t, Z)+\int_{\mathcal{Z}}(f(t, s)-f(t, Z)) \sum_{k \neq Z} G_{Z, k} \delta_{k}(s) d s \\
0 & =\partial_{t} f(t, Z)+\sum_{k \neq Z} G_{Z, k}(f(t, k)-f(t, Z)) \\
0 & =\partial_{t} f(t, Z)+\sum_{k \neq Z} G_{Z, k} f(t, k)-f(t, Z) \sum_{k \neq Z} G_{Z, k} \\
0 & =\partial_{t} f(t, Z)+\sum_{k \neq Z} G_{Z, k} f(t, k)+f(t, Z) G_{Z, Z} \\
0 & =\partial_{t} f(t, Z)+\sum_{k \in \mathcal{Z}} G_{Z, k} f(t, k)
\end{aligned}
$$

Letting $[\boldsymbol{f}(t)]_{Z}=f(t, Z)$, this equation can be written:

$$
\partial_{t} \boldsymbol{f}=-G \boldsymbol{f}
$$

which has solution:

$$
\boldsymbol{f}(t)=e^{G(T-t)} \boldsymbol{f}(T)=e^{G(T-t)} \boldsymbol{\Phi}
$$

If $\Phi(Z)$ is an indicator function of state $k$, then $\boldsymbol{f}(t)$ is a vector of transition probabilities to state $k$ from each other state over a time of $T-t$. This is the same expression which dictates transition probabilities for a continuous time Markov chain with generator matrix 
$G$, and so these definitions are equivalent. 


\section{Appendix B}

\section{Proofs}

\section{B.1 Proofs from Chapter 2}

Proof of Proposition 2. Solving for $g^{ \pm}$: First consider the infimum:

$$
\inf _{g^{ \pm}}\left\{\lambda^{ \pm} e^{g^{ \pm}} e^{-\kappa^{ \pm} \delta^{ \pm}}\left(\delta^{ \pm}+h_{q \mp 1}^{\varphi_{\lambda}}-h_{q}^{\varphi_{\lambda}}\right)+\frac{1}{\varphi_{\lambda}} \lambda^{ \pm}\left(e^{g^{ \pm}}\left(g^{ \pm}-1\right)+1\right)\right\}
$$

First order conditions in $g^{ \pm}$give:

$$
\begin{aligned}
0 & =\lambda^{ \pm} e^{g^{ \pm}} e^{-\kappa^{ \pm} \delta^{ \pm}}\left(\delta^{ \pm}+h_{q \mp 1}^{\varphi_{\lambda}}-h_{q}^{\varphi_{\lambda}}\right)+\frac{\lambda^{ \pm}}{\varphi_{\lambda}} g^{ \pm} e^{g^{ \pm}} \\
g^{ \pm} & =-\varphi_{\lambda} e^{-\kappa^{ \pm} \delta^{ \pm}}\left(\delta^{ \pm}+h_{q \mp 1}^{\varphi_{\lambda}}-h_{q}^{\varphi_{\lambda}}\right)
\end{aligned}
$$

Taking a second derivative with respect to $g^{ \pm}$of the term to be minimized, and evaluating at the value of $g^{ \pm}$which satisfies first order conditions gives:

$$
\begin{aligned}
& \lambda^{ \pm} e^{g^{ \pm}}\left(e^{-\kappa^{ \pm} \delta^{ \pm}}\left(\delta^{ \pm}+h_{q \mp 1}^{\varphi_{\lambda}}-h_{q}^{\varphi_{\lambda}}\right)+\frac{g^{ \pm}+1}{\varphi_{\lambda}}\right) \\
= & \lambda^{ \pm} e^{-\varphi_{\lambda} e^{-\kappa^{ \pm} \delta^{ \pm}}\left(\delta^{ \pm}+h_{q \mp 1}^{\varphi_{\lambda}}-h_{q}^{\varphi_{\lambda}}\right)} \\
> & 0
\end{aligned}
$$

and so $g^{ \pm}$is indeed a minimizer. Clearly, as long as $h_{q \mp 1}^{\varphi_{\lambda}}-h_{q}^{\varphi_{\lambda}}$ is bounded (a result of Proposition 10), $g^{ \pm}$is also bounded and thus admissible. 
Solving for $\delta^{ \pm}$: Substituting this expression for $g^{ \pm}$into equation (2.8) gives equation (2.9). Computing first order conditions for $\delta^{ \pm}$in equation (2.9) gives:

$$
\begin{aligned}
0 & =\frac{\lambda^{ \pm}}{\varphi_{\lambda}} e^{-\varphi_{\lambda} e^{-\kappa^{ \pm} \delta^{ \pm}}\left(\delta^{ \pm}+h_{q \mp 1}^{\varphi_{\lambda}}-h_{q}^{\varphi_{\lambda}}\right)}\left(\varphi_{\lambda} e^{-\kappa^{ \pm} \delta^{ \pm}}-\varphi_{\lambda} \kappa^{ \pm} e^{-\kappa^{ \pm} \delta^{ \pm}}\left(\delta^{ \pm}+h_{q \mp 1}^{\varphi_{\lambda}}-h_{q}^{\varphi_{\lambda}}\right)\right) \\
0 & =\left(\varphi_{\lambda} e^{-\kappa^{ \pm} \delta^{ \pm}}-\varphi_{\lambda} \kappa^{ \pm} e^{-\kappa^{ \pm} \delta^{ \pm}}\left(\delta^{ \pm}+h_{q \mp 1}^{\varphi_{\lambda}}-h_{q}^{\varphi_{\lambda}}\right)\right) \\
0 & =\left(1-\kappa^{ \pm}\left(\delta^{ \pm}+h_{q \mp 1}^{\varphi_{\lambda}}-h_{q}^{\varphi_{\lambda}}\right)\right) \\
\delta^{ \pm} & =\frac{1}{\kappa^{ \pm}}-h_{q \mp 1}^{\varphi_{\lambda}}+h_{q}^{\varphi_{\lambda}}
\end{aligned}
$$

Suppose this expression is non-negative. Then a second derivative of the supremum term in equation (2.9) evaluated at $\delta^{ \pm}$computed above gives:

$$
-\lambda^{ \pm} e^{\frac{-\varphi_{\lambda}}{\kappa^{ \pm}} e^{\kappa^{ \pm}\left(h_{q \mp 1}^{\varphi}-h_{q}^{\varphi} \lambda\right)}} \kappa^{ \pm} e^{\kappa^{ \pm}\left(h_{q \mp 1}^{\varphi}-h_{q}^{\varphi}\right)}<0
$$

and so the computed $\delta^{ \pm}$is indeed a minimizer. If on the other hand the feedback expression for $\delta^{ \pm}$is negative, then compute the first derivative of the supremum term at $\delta^{ \pm}=0$ to see that:

$$
\begin{aligned}
& \frac{\lambda^{ \pm}}{\varphi_{\lambda}} e^{-\varphi_{\lambda} e^{-\kappa^{ \pm} \delta^{ \pm}}\left(\delta^{ \pm}+h_{q \mp 1}^{\varphi_{\lambda}}-h_{q}^{\varphi_{\lambda}}\right)}\left(\varphi_{\lambda} e^{-\kappa^{ \pm} \delta^{ \pm}}-\varphi_{\lambda} \kappa^{ \pm} e^{-\kappa^{ \pm} \delta^{ \pm}}\left(\delta^{ \pm}+h_{q \mp 1}^{\varphi_{\lambda}}-h_{q}^{\varphi_{\lambda}}\right)\right) \\
= & \lambda^{ \pm} e^{-\varphi_{\lambda}\left(h_{q \mp 1}^{\varphi_{\lambda}}-h_{q}^{\varphi_{\lambda}}\right)}\left(1-\kappa^{ \pm}\left(h_{q \mp 1}^{\varphi_{\lambda}}-h_{q}^{\varphi_{\lambda}}\right)\right) \\
< & 0
\end{aligned}
$$

which follows from $\frac{1}{\kappa^{ \pm}}-h_{q \mp 1}^{\varphi_{\lambda}}+h_{q}^{\varphi_{\lambda}}<0$. And so the optimal $\delta^{ \pm}$is $\left(\frac{1}{\kappa^{ \pm}}+h_{q \mp 1}^{\varphi_{\lambda}}-h_{q}^{\varphi_{\lambda}}\right)_{+}$as desired.

Proof of Proposition 3. Solving for $g^{ \pm}$: First consider the infimum:

$$
\begin{aligned}
\inf _{g^{ \pm} \in \mathcal{G}^{ \pm}}\left\{\lambda^{ \pm}[\right. & \left.\int_{\delta^{ \pm}}^{\infty} e^{g^{ \pm}(y)} F^{ \pm}(y) d y\right]\left(\delta^{ \pm}+h_{q \mp 1}^{\varphi_{\kappa}}-h_{q}^{\varphi_{\kappa}}\right) \\
& \left.+\frac{1}{\varphi_{\kappa}} \lambda^{ \pm} \int_{0}^{\infty}\left(e^{g^{ \pm}(y)}\left(g^{ \pm}(y)-1\right)+1\right) F^{ \pm}(y) d y\right\}
\end{aligned}
$$

Recall that the sets $\mathcal{G}^{ \pm}$are defined as:

$$
\mathcal{G}=\left\{g: \int_{0}^{\infty} e^{g(y)} F(y) d y=1\right\} .
$$


In order to perform the optimization with the constraint $g^{ \pm} \in \mathcal{G}^{ \pm}$, introduce a Lagrange multiplier $\gamma^{ \pm}$in the form of an additional term to the infimum:

$$
\begin{aligned}
& \inf _{g^{ \pm}}\left\{\lambda^{ \pm}\left[\int_{\delta^{ \pm}}^{\infty} e^{g^{ \pm}(y)} F^{ \pm}(y) d y\right]\left(\delta^{ \pm}+h_{q \mp 1}^{\varphi_{\kappa}}-h_{q}^{\varphi_{\kappa}}\right)\right. \\
+ & \left.\frac{1}{\varphi_{\kappa}} \lambda^{ \pm} \int_{0}^{\infty}\left(e^{g^{ \pm}(y)}\left(g^{ \pm}(y)-1\right)+1\right) F^{ \pm}(y) d y+\gamma^{ \pm}\left(\int_{0}^{\infty} e^{g^{ \pm}(y)} F^{ \pm}(y) d y-1\right)\right\}
\end{aligned}
$$

The infimum over $g^{ \pm}$is now unrestricted. Since the infimum is to be taken over all functions $g^{ \pm}(y)$, and the term to be minimized only has integrals of functions of $g^{ \pm}(y)$, the minimization with respect to $g^{ \pm}$can be accomplished by minimizing the integrand for each $y \in[0, \infty)$. This is done in each of two domains $\left(y \in\left[0, \delta^{ \pm}\right]\right.$and $\left.y \in\left(\delta^{ \pm}, \infty\right)\right)$ separately. For $y \in\left(\delta^{ \pm}, \infty\right)$, consider the minimization with respect to $g^{ \pm}$:

$$
\inf _{g^{ \pm}}\left\{\lambda^{ \pm} e^{g^{ \pm}(y)}\left(\delta^{ \pm}+h_{q \mp 1}^{\varphi_{\kappa}}-h_{q}^{\varphi_{\kappa}}\right)+\frac{1}{\varphi_{\kappa}} \lambda^{ \pm}\left(e^{g^{ \pm}(y)}\left(g^{ \pm}(y)-1\right)+1\right)+\gamma^{ \pm}\left(e^{g^{ \pm}(y)}-1\right)\right\}
$$

First order conditions give:

$$
\begin{aligned}
& \lambda^{ \pm} e^{g^{ \pm}(y)}\left(\delta^{ \pm}+h_{q \mp 1}^{\varphi_{\kappa}}-h_{q}^{\varphi_{\kappa}}\right)+\frac{\lambda^{ \pm}}{\varphi_{\lambda}} g^{ \pm}(y) e^{g^{ \pm}(y)}+\gamma^{ \pm} e^{g^{ \pm}(y)}=0 \\
& g^{ \pm}(y)=-\frac{\gamma^{ \pm} \varphi_{\kappa}}{\lambda^{ \pm}}-\varphi_{\kappa}\left(\delta^{ \pm}+h_{q \mp 1}^{\varphi_{\kappa}}-h_{q}^{\varphi_{\kappa}}\right)
\end{aligned}
$$

And for $y \in\left[0, \delta^{ \pm}\right]$:

$$
\inf _{g^{ \pm}}\left\{\frac{1}{\varphi_{\kappa}} \lambda^{ \pm}\left(e^{g^{ \pm}(y)}\left(g^{ \pm}(y)-1\right)+1\right)+\gamma^{ \pm}\left(e^{g^{ \pm}(y)}-1\right)\right\}
$$

First order conditions imply:

$$
g^{ \pm}(y)=-\frac{\gamma^{ \pm} \varphi_{\kappa}}{\lambda^{ \pm}}
$$

combining equations (B.3) and (B.4) give:

$$
g^{ \pm}(y)=-\frac{\gamma^{ \pm} \varphi_{\kappa}}{\lambda^{ \pm}}-\varphi_{\kappa}\left(\delta^{ \pm}+h_{q \mp 1}^{\varphi_{\kappa}}-h_{q}^{\varphi_{\kappa}}\right) \mathbb{1}_{y>\delta^{ \pm}}
$$

Solving for $\gamma^{ \pm}$: Now substitute this expression for $g^{ \pm}(y)$ into the constraint $\int_{0}^{\infty} e^{g^{ \pm}(y)} F^{ \pm}(y) d y=$ 
1 in order to solve for the Lagrange multiplier $\gamma^{ \pm}$:

$$
\begin{aligned}
1 & =\int_{0}^{\infty} e^{g^{ \pm}(y)} F^{ \pm}(y) d y \\
1 & =\int_{0}^{\delta^{ \pm}} e^{g^{ \pm}(y)} F^{ \pm}(y) d y+\int_{\delta^{ \pm}}^{\infty} e^{g^{ \pm}(y)} F^{ \pm}(y) d y \\
1 & =e^{-\frac{\gamma^{ \pm} \varphi_{\kappa}}{\lambda^{ \pm}}} \int_{0}^{\delta^{ \pm}} F^{ \pm}(y) d y+e^{-\frac{\gamma^{ \pm} \varphi_{\kappa}}{\lambda^{ \pm}}-\varphi_{\kappa}\left(\delta^{ \pm}+h_{q \mp 1}^{\varphi_{\kappa}}-h_{q}^{\varphi_{\kappa}}\right)} \int_{\delta^{ \pm}}^{\infty} F^{ \pm}(y) d y \\
e^{\frac{\gamma^{ \pm} \varphi_{\kappa}}{\lambda^{ \pm}}} & =1-e^{-\kappa^{ \pm} \delta^{ \pm}}+e^{-\varphi_{\kappa}\left(\delta^{ \pm}+h_{q \mp 1}^{\varphi_{\kappa}}-h_{q}^{\varphi_{\kappa}}\right)} e^{-\kappa^{ \pm} \delta^{ \pm}} \\
\gamma^{ \pm} & =\frac{\lambda^{ \pm}}{\varphi_{\kappa}} \log \left(1-e^{-\kappa^{ \pm} \delta^{ \pm}}+e^{-\varphi_{\kappa}\left(\delta^{ \pm}+h_{q \mp 1}^{\varphi_{\kappa}}-h_{q}^{\varphi_{\kappa}}\right)} e^{-\kappa^{ \pm} \delta^{ \pm}}\right)
\end{aligned}
$$

Substituting this expression for $\gamma^{ \pm}$into equation (B.5) gives:

$$
\begin{aligned}
& g^{ \pm}(y)=-\log \left(1-e^{-\kappa^{ \pm} \delta^{ \pm}}+e^{-\varphi_{\kappa}\left(\delta^{ \pm}+h_{q \mp 1}^{\varphi_{\kappa}}-h_{q}^{\varphi_{\kappa}}\right)} e^{-\kappa^{ \pm} \delta^{ \pm}}\right) \\
& -\varphi_{\kappa}\left(\delta^{ \pm}+h_{q \mp 1}^{\varphi_{\kappa}}-h_{q}^{\varphi_{\kappa}}\right) \mathbb{1}_{y>\delta^{ \pm}}
\end{aligned}
$$

This expression of $g^{ \pm}(y)$ is bounded, and thus admissible, as long as $h_{q \mp 1}^{\varphi_{\kappa}}-h_{q}^{\varphi_{\kappa}}$ is bounded (a consequence of Proposition 10).

Verify that $g^{ \pm}$provides a minimum: To show that this expression for $g^{ \pm}(y)$ is indeed a minimizer, proceed as follows: let $f(y)$ be any other function in $\mathcal{G}^{ \pm}$. Define $k(y)=$ $e^{f(y)}-e^{g^{ \pm}(y)}$. Now define $f_{\epsilon}(y)=\log \left(\epsilon k(y)+e^{g^{ \pm}(y)}\right)$ for $\epsilon \in[0,1]$. The parametrization of functions $f_{\epsilon}(y)$ satisfies $f_{0}(y)=g^{ \pm}(y), f_{1}(y)=f(y)$, and $f_{\epsilon} \in \mathcal{G}^{ \pm}$. To show the last claim:

$$
\begin{aligned}
\int_{0}^{\infty} e^{f_{\epsilon}(y)} F^{ \pm}(y) d y & =\int_{0}^{\infty} \epsilon k(y)+e^{g^{ \pm}(y)} F^{ \pm}(y) d y \\
& =\int_{0}^{\infty} \epsilon\left(e^{f(y)}-e^{g^{ \pm}(y)}\right) F^{ \pm}(y) d y+\int_{0}^{\infty} e^{g^{ \pm}(y)} F^{ \pm}(y) d y \\
& =\int_{0}^{\infty} e^{g^{ \pm}(y)} F^{ \pm}(y) d y \\
& =1
\end{aligned}
$$

Now, evaluate the term to be minimized in (B.2) at $f_{\epsilon}(y)$ :

$$
\lambda^{ \pm}\left[\int_{\delta^{ \pm}}^{\infty} e^{f_{\epsilon}(y)} F^{ \pm}(y) d y\right]\left(\delta^{ \pm}+h_{q \mp 1}^{\varphi_{\kappa}}-h_{q}^{\varphi_{\kappa}}\right)+\frac{1}{\varphi_{\kappa}} \lambda^{ \pm} \int_{0}^{\infty}\left(e^{f_{\epsilon}(y)}\left(f_{\epsilon}(y)-1\right)+1\right) F^{ \pm}(y) d y
$$

Derivatives of this expression with respect to $\epsilon$ will be taken. The first derivative will 
vanish at $\epsilon=0$, as should be expected. The second derivative will be positive for all $\epsilon \in[0,1]$, showing that $g^{ \pm}(y)$ is indeed a minimizer. Expanding the expression in terms of $g^{ \pm}(y)$ and $k(y)$ gives:

$$
\begin{gathered}
\lambda^{ \pm}\left[\int_{\delta^{ \pm}}^{\infty}\left(e^{g^{ \pm}(y)}+\epsilon k(y)\right) F^{ \pm}(y) d y\right]\left(\delta^{ \pm}+h_{q \mp 1}^{\varphi_{\kappa}}-h_{q}^{\varphi_{\kappa}}\right) \\
+\frac{1}{\varphi_{\kappa}} \lambda^{ \pm} \int_{0}^{\infty}\left(\left(e^{g^{ \pm}(y)}+\epsilon k(y)\right)\left(\log \left(e^{g^{ \pm}(y)}+\epsilon k(y)\right)-1\right)+1\right) F^{ \pm}(y) d y
\end{gathered}
$$

Taking a first derivative with respect to $\epsilon$ :

$$
\begin{gathered}
\lambda^{ \pm}\left[\int_{\delta^{ \pm}}^{\infty} k(y) F^{ \pm}(y) d y\right]\left(\delta^{ \pm}+h_{q \mp 1}^{\varphi_{\kappa}}-h_{q}^{\varphi_{\kappa}}\right) \\
+\frac{1}{\varphi_{\kappa}} \lambda^{ \pm} \int_{0}^{\infty} k(y)\left(\log \left(e^{g^{ \pm}(y)}+\epsilon k(y)\right)-1\right) F^{ \pm}(y) d y+\frac{1}{\varphi_{\kappa}} \lambda^{ \pm} \int_{0}^{\infty} k(y) F^{ \pm}(y) d y \\
=\lambda^{ \pm}\left[\int_{\delta^{ \pm}}^{\infty} k(y) F^{ \pm}(y) d y\right]\left(\delta^{ \pm}+h_{q \mp 1}^{\varphi_{\kappa}}-h_{q}^{\varphi_{\kappa}}\right)+\frac{1}{\varphi_{\kappa}} \lambda^{ \pm} \int_{0}^{\infty} k(y) \log \left(e^{g^{ \pm}(y)}+\epsilon k(y)\right) F^{ \pm}(y) d y
\end{gathered}
$$

Let:

$$
\begin{aligned}
& \bar{g}^{ \pm}=-\log \left(A^{ \pm}\right)-\varphi_{\kappa}\left(\delta^{ \pm}+h_{q \mp 1}^{\varphi_{\kappa}}-h_{q}^{\varphi_{\kappa}}\right) \\
& \underline{g}^{ \pm}=-\log \left(A^{ \pm}\right) \\
& A^{ \pm}=1-e^{-\kappa^{ \pm} \delta^{ \pm}}+e^{-\varphi_{\kappa}\left(\delta^{ \pm}+h_{q \mp 1}^{\varphi_{\kappa}}-h_{q}^{\varphi_{\kappa}}\right)} e^{-\kappa^{ \pm} \delta^{ \pm}}
\end{aligned}
$$

Then evaluating the first derivative expression at $\epsilon=0$ gives:

$$
\begin{aligned}
& \lambda^{ \pm}\left[\int_{\delta^{ \pm}}^{\infty} k(y) F^{ \pm}(y) d y\right]\left(\delta^{ \pm}+h_{q \mp 1}^{\varphi_{\kappa}}-h_{q}^{\varphi_{\kappa}}\right)+\frac{1}{\varphi_{\kappa}} \lambda^{ \pm} \int_{0}^{\infty} k(y) g^{ \pm}(y) F^{ \pm}(y) d y \\
& =\lambda^{ \pm}\left[\int_{\delta^{ \pm}}^{\infty} k(y) F^{ \pm}(y) d y\right]\left(\delta^{ \pm}+h_{q \mp 1}^{\varphi_{\kappa}}-h_{q}^{\varphi_{\kappa}}\right)+\frac{1}{\varphi_{\kappa}} \lambda^{ \pm} \int_{0}^{\delta^{ \pm}} k(y) \underline{g}^{ \pm} F^{ \pm}(y) d y \\
& +\frac{1}{\varphi_{\kappa}} \lambda^{ \pm} \int_{\delta^{ \pm}}^{\infty} k(y) \bar{g}^{ \pm} F^{ \pm}(y) d y \\
& =\lambda^{ \pm}\left[\int_{\delta^{ \pm}}^{\infty} k(y) F^{ \pm}(y) d y\right]\left(\delta^{ \pm}+h_{q \mp 1}^{\varphi_{\kappa}}-h_{q}^{\varphi_{\kappa}}\right)-\log \left(A^{ \pm}\right) \frac{1}{\varphi_{\kappa}} \lambda^{ \pm} \int_{0}^{\infty} k(y) F^{ \pm}(y) d y \\
& -\left(\delta^{ \pm}+h_{q \mp 1}^{\varphi_{\kappa}}-h_{q}^{\varphi_{\kappa}}\right) \lambda^{ \pm} \int_{\delta^{ \pm}}^{\infty} k(y) F^{ \pm}(y) d y \\
& =0
\end{aligned}
$$


as expected. Now, taking a second derivative with respect to $\epsilon$ yields:

$$
\frac{1}{\varphi_{\kappa}} \lambda^{ \pm} \int_{0}^{\infty} \frac{k^{2}(y)}{e^{g^{ \pm}(y)}+\epsilon k(y)} F^{ \pm}(y) d y=\frac{1}{\varphi_{\kappa}} \lambda^{ \pm} \int_{0}^{\infty} \frac{k^{2}(y)}{e^{f_{\epsilon}(y)}} F^{ \pm}(y) d y
$$

This expression is positive for all $\epsilon \in[0,1]$, proving that $g^{ \pm}(y)$ in equation (B.6) is indeed a minimizer.

Solving for $\delta^{ \pm}$: Continuing now to find the optimal $\delta^{ \pm}$, the expression for $g^{ \pm}(y)$ from equation (B.6) is substituted into equation (B.2), and performing some tedious computations gives the term to be maximized with respect to $\delta^{ \pm}$:

$$
\sup _{\delta^{ \pm} \geq 0}\left\{-\frac{\lambda^{ \pm}}{\varphi_{\kappa}} \log \left(1-e^{-\kappa^{ \pm} \delta^{ \pm}}+e^{-\kappa^{ \pm} \delta^{ \pm}-\varphi_{\kappa}\left(\delta^{ \pm}+h_{q+1}^{\varphi_{\kappa}}-h_{q}^{\varphi_{\kappa}}\right)}\right)\right\}
$$

Solving for the maximizing $\delta^{ \pm}$here is equivalent to solving for the minimizing $\delta^{ \pm}$of:

$$
\inf _{\delta^{ \pm} \geq 0}\left\{1-e^{-\kappa^{ \pm} \delta^{ \pm}}+e^{-\kappa^{ \pm} \delta^{ \pm}-\varphi_{\kappa}\left(\delta^{ \pm}+h_{q \neq 1}^{\varphi_{\kappa}}-h_{q}^{\varphi_{\kappa}}\right)}\right\}
$$

First order conditions of this expression give:

$$
\begin{aligned}
0 & =\kappa^{ \pm} e^{-\kappa^{ \pm} \delta^{ \pm}}-\left(\kappa^{ \pm}+\varphi_{\kappa}\right) e^{-\kappa^{ \pm} \delta^{ \pm}-\varphi_{\kappa}\left(\delta^{ \pm}+h_{q \mp 1}^{\varphi_{\kappa}}-h_{q}^{\varphi_{\kappa}}\right)} \\
0 & =\kappa^{ \pm}-\left(\kappa^{ \pm}+\varphi_{\kappa}\right) e^{-\varphi_{\kappa}\left(\delta^{ \pm}+h_{q \mp 1}^{\varphi_{\kappa}}-h_{q}^{\varphi_{\kappa}}\right)} \\
\delta^{ \pm} & =\frac{1}{\varphi_{\kappa}} \log \left(1+\frac{\varphi_{\kappa}}{\kappa^{ \pm}}\right)-h_{q \mp 1}^{\varphi_{\kappa}}+h_{q}^{\varphi_{\kappa}}
\end{aligned}
$$

Suppose this expression is positive. Then the second derivative of the term to be minimized, evaluated at this value of $\delta^{ \pm}$, is:

$$
\begin{aligned}
& -\kappa^{ \pm 2} e^{-\kappa^{ \pm} \delta^{ \pm}}+\left(\kappa^{ \pm}+\varphi_{\kappa}\right)^{2} e^{-\kappa^{ \pm} \delta^{ \pm}-\varphi_{\kappa}\left(\delta^{ \pm}+h_{q \mp 1}^{\varphi_{\kappa}}-h_{q}^{\varphi_{\kappa}}\right)} \\
= & e^{-\kappa^{ \pm} \delta^{ \pm}}\left(\left(\kappa^{ \pm}+\varphi_{\kappa}\right)^{2} \frac{\kappa^{ \pm}}{\kappa^{ \pm}+\varphi_{\kappa}}-\kappa^{ \pm 2}\right) \\
= & \kappa^{ \pm} \varphi_{\kappa} e^{-\kappa^{ \pm} \delta^{ \pm}} \\
> & 0
\end{aligned}
$$

and so this value of $\delta^{ \pm}$is indeed a maximizer equation (B.7). Now suppose the given expression for $\delta^{ \pm}$is negative. Then the first derivative computed above and evaluated at 
$\delta^{ \pm}=0$ gives:

$$
\begin{aligned}
& \kappa^{ \pm} e^{-\kappa^{ \pm} \delta^{ \pm}}-\left(\kappa^{ \pm}+\varphi_{\kappa}\right) e^{-\kappa^{ \pm} \delta^{ \pm}-\varphi_{\kappa}\left(\delta^{ \pm}+h_{q+1}^{\varphi_{\kappa}}-h_{q}^{\varphi_{\kappa}}\right)} \\
= & \kappa^{ \pm}-\left(\kappa^{ \pm}+\varphi_{\kappa}\right) e^{-\varphi_{\kappa}\left(h_{q+1}^{\varphi_{\kappa}}-h_{q}^{\varphi_{\kappa}}\right)} \\
> & 0
\end{aligned}
$$

which follows from $\frac{1}{\varphi_{\kappa}} \log \left(1+\frac{\varphi_{\kappa}}{\kappa^{ \pm}}\right)-h_{q \mp 1}^{\varphi_{\kappa}}+h_{q}^{\varphi_{\kappa}}<0$. Thus, $\delta^{ \pm}=0$ is a maximizer of equation (B.7) when the feedback expression is negative. This gives

$$
\delta^{ \pm}=\left(\frac{1}{\varphi_{\kappa}} \log \left(1+\frac{\varphi_{\kappa}}{\kappa^{ \pm}}\right)-h_{q \mp 1}^{\varphi_{\kappa}}+h_{q}^{\varphi_{\kappa}}\right)_{+}
$$

as desired.

Proof of Proposition 7. The minimization in $\eta$ is independent of the optimization in $\delta^{ \pm}, g^{ \pm \lambda}$, and $g^{ \pm \kappa}$ and identical to that of Proposition 4. For the minimization in $g^{ \pm \lambda}$ and $g^{ \pm \kappa}$, first consider $\varphi_{\lambda}>\varphi_{\kappa}$. Then the term to be minimized is:

$$
\begin{aligned}
& \lambda^{ \pm}\left[\int_{\delta^{ \pm}}^{\infty} e^{g^{ \pm \lambda}+g^{ \pm \kappa}(y)} F^{ \pm}(y) d y\right]\left(\delta^{ \pm}+h_{q \mp 1}^{\psi}-h_{q}^{\psi}\right)+\mathcal{K}_{ \pm}^{\varphi_{\lambda}, \varphi_{\kappa}}\left(g^{ \pm \lambda}, g^{ \pm \kappa}\right) \\
= & \lambda^{ \pm}\left[\int_{\delta^{ \pm}}^{\infty} e^{g^{\lambda \pm}+g^{\kappa \pm}(y)} F^{ \pm}(y) d y\right]\left(\delta^{ \pm}+h_{q \mp 1}^{\psi}-h_{q}^{\psi}\right) \\
& +\frac{1}{\varphi_{\lambda}}\left[\lambda^{ \pm} \int_{0}^{\infty}-\left(e^{g^{\lambda \pm}}-1\right)+g^{\lambda \pm} e^{g^{\lambda \pm}+g^{\kappa \pm}(y)} F^{ \pm}(y) d y\right] \\
& +\frac{1}{\varphi_{\kappa}}\left[\lambda^{ \pm} \int_{0}^{\infty}-\left(e^{g^{\kappa \pm}(y)}-1\right) e^{g^{\lambda \pm}}+g^{\kappa \pm}(y) e^{g^{\lambda \pm}+g^{\kappa \pm}(y)} F^{ \pm}(y) d y\right]
\end{aligned}
$$

Solving for $g^{ \pm \kappa}$ : The constraint $\int_{0}^{\infty} e^{g^{ \pm \kappa}(y)} F^{ \pm}(y) d y=1$ is again handled by introducing Lagrange multipliers $\gamma^{ \pm}$and then minimizing over unconstrained $g^{ \pm \kappa}$. The optimization with respect to $g^{ \pm \kappa}$ is also handled in a pointwise fashion by minimizing the integrand as in the proof of Proposition 3. For $y \in\left(\delta^{ \pm}, \infty\right)$, the quantity to be minimized is:

$$
\begin{aligned}
\lambda^{ \pm} e^{g^{\lambda \pm}+g^{\kappa \pm}}(y) & \left(\delta^{ \pm}+h_{q \mp 1}^{\psi}-h_{q}^{\psi}\right)+\frac{\lambda^{ \pm}}{\varphi_{\lambda}}\left(-\left(e^{g^{\lambda \pm}}-1\right)+g^{\lambda \pm} e^{g^{\lambda \pm}+g^{\kappa \pm}}(y)\right) \\
+ & \frac{\lambda^{ \pm}}{\varphi_{\kappa}}\left(-\left(e^{g^{\kappa \pm}(y)}-1\right) e^{g^{\lambda \pm}}+g^{\kappa \pm}(y) e^{g^{\lambda \pm}+g^{\kappa \pm}(y)}\right)+\gamma^{ \pm}\left(e^{g^{\kappa \pm}(y)}-1\right)
\end{aligned}
$$


First order conditions in $g^{ \pm \kappa}(y)$ give:

$$
\begin{gathered}
0=\lambda^{ \pm} e^{g^{\lambda^{ \pm}}+g^{\kappa \pm}(y)}\left(\delta^{ \pm}+h_{q \mp 1}^{\psi}-h_{q}^{\psi}\right)+\frac{\lambda^{ \pm}}{\varphi_{\lambda}} g^{ \pm \lambda} e^{g^{ \pm \lambda}+g^{ \pm \kappa}(y)} \\
+\frac{\lambda^{ \pm}}{\varphi_{\kappa}} g^{ \pm \kappa}(y) e^{g^{ \pm \lambda}+g^{ \pm \kappa}(y)}+\gamma^{ \pm} e^{g^{ \pm \kappa}(y)} \\
0=\lambda^{ \pm} e^{g^{\lambda \pm}}\left(\delta^{ \pm}+h_{q \mp 1}^{\psi}-h_{q}^{\psi}\right)+\frac{\lambda^{ \pm}}{\varphi_{\lambda}} g^{ \pm \lambda} e^{g^{ \pm \lambda}}+\frac{\lambda^{ \pm}}{\varphi_{\kappa}} g^{ \pm \kappa}(y) e^{g^{ \pm \lambda}}+\gamma^{ \pm} \\
g^{ \pm \kappa}(y)=-\frac{\varphi_{\kappa}}{\varphi_{\lambda}} g^{ \pm \lambda}-\frac{\gamma^{ \pm} \varphi_{\kappa}}{\lambda^{ \pm}} e^{-g^{ \pm \lambda}}-\varphi_{\kappa}\left(\delta^{ \pm}+h_{q \mp 1}^{\psi}-h_{q}^{\psi}\right)
\end{gathered}
$$

Similarly, first order conditions in $g^{ \pm \kappa}(y)$ for $y \in\left[0, \delta^{ \pm}\right]$give:

$$
g^{ \pm \kappa}(y)=-\frac{\varphi_{\kappa}}{\varphi_{\lambda}} g^{ \pm \lambda}-\frac{\gamma^{ \pm} \varphi_{\kappa}}{\lambda^{ \pm}} e^{-g^{ \pm \lambda}}
$$

Combining equations (B.10) and (B.11) gives:

$$
g^{ \pm \kappa}(y)=-\frac{\varphi_{\kappa}}{\varphi_{\lambda}} g^{ \pm \lambda}-\frac{\gamma^{ \pm} \varphi_{\kappa}}{\lambda^{ \pm}} e^{-g^{ \pm \lambda}}-\varphi_{\kappa}\left(\delta^{ \pm}+h_{q \mp 1}^{\psi}-h_{q}^{\psi}\right) \mathbb{1}_{y>\delta^{ \pm}}
$$

Solving for $\gamma^{ \pm}$: Substituting this expression into the integral constraint and performing some computations gives an expression for $\gamma^{ \pm}$:

$$
\begin{aligned}
& 1=\int_{0}^{\infty} e^{g^{ \pm \kappa}(y)} F^{ \pm}(y) d y \\
& 1=\int_{0}^{\delta^{ \pm}} e^{g^{ \pm \kappa}(y)} F^{ \pm}(y) d y+\int_{\delta^{ \pm}}^{\infty} e^{g^{ \pm \kappa}(y)} F^{ \pm}(y) d y \\
& 1=e^{-\frac{\varphi_{\kappa}}{\varphi_{\lambda}} g^{ \pm \lambda}-\frac{\gamma^{ \pm} \varphi_{\kappa}}{\lambda^{ \pm}} e^{-g^{ \pm \lambda}}} \int_{0}^{\delta^{ \pm}} F^{ \pm}(y) d y \\
& +e^{-\frac{\varphi_{\kappa}}{\varphi_{\lambda}} g^{ \pm \lambda}-\frac{\gamma^{ \pm} \varphi_{\kappa}}{\lambda \pm} e^{-g^{ \pm \lambda}}-\varphi_{\kappa}\left(\delta^{ \pm}+h_{q \mp 1}^{\varphi_{\kappa}}-h_{q}^{\varphi_{\kappa}}\right)} \int_{\delta^{ \pm}}^{\infty} F^{ \pm}(y) d y \\
& e^{\frac{\gamma^{ \pm} \varphi_{\kappa}}{\lambda \pm} e^{-g^{ \pm \lambda}}}=e^{-\frac{\varphi_{\kappa}}{\varphi_{\lambda}} g^{ \pm \lambda}}\left(1-e^{-\kappa^{ \pm} \delta^{ \pm}}+e^{-\varphi_{\kappa}\left(\delta^{ \pm}+h_{q \mp 1}^{\varphi_{\kappa}}-h_{q}^{\varphi_{\kappa}}\right)} e^{-\kappa^{ \pm} \delta^{ \pm}}\right) \\
& \gamma^{ \pm}=-\frac{g^{ \pm \lambda} \lambda^{ \pm}}{\varphi_{\lambda}} e^{g^{ \pm \lambda}}+\frac{\lambda^{ \pm} e^{g^{ \pm \lambda}}}{\varphi_{\kappa}} \log \left(1-e^{-\kappa^{ \pm} \delta^{ \pm}}+e^{-\varphi_{\kappa}\left(\delta^{ \pm}+h_{q+1}^{\varphi_{\kappa}}-h_{q}^{\varphi_{\kappa}}\right)} e^{-\kappa^{ \pm} \delta^{ \pm}}\right)
\end{aligned}
$$

Substituting this into equation (B.12) gives:

$$
\begin{gathered}
g^{ \pm \kappa}(y)=-\log \left(1-e^{-\kappa^{ \pm} \delta^{ \pm}}+e^{-\varphi_{\kappa}\left(\delta^{ \pm}+h_{q \mp 1}^{\varphi_{\kappa}}-h_{q}^{\varphi_{\kappa}}\right)} e^{-\kappa^{ \pm} \delta^{ \pm}}\right) \\
-\varphi_{\kappa}\left(\delta^{ \pm}+h_{q \mp 1}^{\psi}-h_{q}^{\psi}\right) \mathbb{1}_{y>\delta^{ \pm}}
\end{gathered}
$$


Note that $g^{ \pm \kappa}(y)$ is bounded as long as $h_{q \mp 1}^{\psi}-h_{q}^{\psi}$ is bounded (a consequence of Proposition 10 ), and thus is admissible.

Verify that $g^{ \pm \kappa}$ provides a minimum: To prove that this expression for $g^{ \pm \kappa}(y)$ is indeed a minimum, proceed in a similar fashion to the proof of Proposition 3. First, it will be convenient to introduce some shorthand notation:

$$
\begin{aligned}
\Delta^{ \pm} & =h_{q \mp 1}^{\varphi_{\kappa}}-h_{q}^{\varphi_{\kappa}} \\
A^{ \pm} & =1-e^{-\kappa^{ \pm} \delta^{ \pm}}+e^{-\varphi_{\kappa}\left(\delta^{ \pm}+\Delta^{ \pm}\right)} e^{-\kappa^{ \pm} \delta^{ \pm}} \\
\underline{g}^{ \pm} & =-\log A^{ \pm} \\
\bar{g}^{ \pm} & =-\log A^{ \pm}-\varphi_{\kappa}\left(\delta^{ \pm}+\Delta^{ \pm}\right)
\end{aligned}
$$

It is important to note that none of these quantities depends on $g^{ \pm \lambda}$. Also note that $\underline{g}^{ \pm}$ and $\bar{g}^{ \pm}$are the two possible values that $g^{ \pm \kappa}(y)$ can take. Let $f$ be any other function in $\mathcal{G}^{ \pm}$and define $k(y)=e^{f(y)}-e^{g^{ \pm \kappa}(y)}$. Then define $f_{\epsilon}(y)=\log \left(\epsilon k(y)+e^{g^{ \pm \kappa}(y)}\right)$. Evaluating the term to be minimized at $f_{\epsilon}$ gives:

$$
\begin{aligned}
& \lambda^{ \pm}\left[\int_{\delta^{ \pm}}^{\infty} e^{g^{\lambda \pm}+f_{\epsilon}(y)} F^{ \pm}(y) d y\right]\left(\delta^{ \pm}+\Delta^{ \pm}\right) \\
+ & \frac{1}{\varphi_{\lambda}}\left[\lambda^{ \pm} \int_{0}^{\infty}-\left(e^{g^{\lambda \pm}}-1\right)+g^{\lambda \pm} e^{g^{\lambda \pm}+f_{\epsilon}(y)} F^{ \pm}(y) d y\right] \\
& +\frac{1}{\varphi_{\kappa}}\left[\lambda^{ \pm} \int_{0}^{\infty}-\left(e^{f_{\epsilon}(y)}-1\right) e^{g^{\lambda \pm}}+f_{\epsilon}(y) e^{g^{\lambda \pm}+f_{\epsilon}(y)} F^{ \pm}(y) d y\right] \\
= & \lambda^{ \pm}\left[\int_{\delta^{ \pm}}^{\infty} e^{g^{\lambda \pm}}\left(e^{\bar{g}^{ \pm}}+\epsilon k(y)\right) F^{ \pm}(y) d y\right]\left(\delta^{ \pm}+\Delta^{ \pm}\right) \\
& +\frac{1}{\varphi_{\lambda}}\left[\lambda^{ \pm} \int_{0}^{\delta^{ \pm}}-\left(e^{g^{\lambda \pm}}-1\right)+g^{\lambda \pm} e^{g^{\lambda \pm}}\left(e^{\underline{g}^{ \pm}}+\epsilon k(y)\right) F^{ \pm}(y) d y\right] \\
& +\frac{1}{\varphi_{\lambda}}\left[\lambda^{ \pm} \int_{\delta^{ \pm}}^{\infty}-\left(e^{g^{\lambda \pm}}-1\right)+g^{\lambda \pm} e^{g^{\lambda \pm}}\left(e^{\bar{g}^{ \pm}}+\epsilon k(y)\right) F^{ \pm}(y) d y\right] \\
& +\frac{1}{\varphi_{\kappa}}\left[\lambda^{ \pm} \int_{0}^{\delta^{ \pm}}-\left(e^{g^{ \pm}}+\epsilon k(y)-1\right) e^{g^{\lambda \pm}}+\log \left(e^{g^{ \pm}}+\epsilon k(y)\right) e^{g^{\lambda \pm}}\left(e^{g^{ \pm}}+\epsilon k(y)\right) F^{ \pm}(y) d y\right] \\
& +\frac{1}{\varphi_{\kappa}}\left[\lambda^{ \pm} \int_{\delta^{ \pm}}^{\infty}-\left(e^{\bar{g}^{ \pm}}+\epsilon k(y)-1\right) e^{g^{\lambda \pm}}+\log \left(e^{\bar{g}^{ \pm}}+\epsilon k(y)\right) e^{g^{\lambda \pm}}\left(e^{\bar{g}^{ \pm}}+\epsilon k(y)\right) F^{ \pm}(y) d y\right]
\end{aligned}
$$


and taking a derivative with respect to $\epsilon$ gives:

$$
\begin{aligned}
& \lambda^{ \pm}\left[\int_{\delta^{ \pm}}^{\infty} e^{g^{\lambda \pm}} k(y) F(y) d y\right]\left(\delta^{ \pm}+\Delta^{ \pm}\right)+\frac{1}{\varphi_{\lambda}}\left[\lambda^{ \pm} \int_{0}^{\infty} g^{\lambda \pm} e^{g^{\lambda \pm}} k(y) F^{ \pm}(y) d y\right] \\
& +\frac{1}{\varphi_{\kappa}}\left[\lambda^{ \pm} \int_{0}^{\delta^{ \pm}}-k(y) e^{g^{\lambda \pm}}+k(y) e^{g^{\lambda \pm}}+k(y) \log \left(e^{g^{ \pm}}+\epsilon k(y)\right) e^{g^{\lambda \pm}} F^{ \pm}(y) d y\right] \\
& +\frac{1}{\varphi_{\kappa}}\left[\lambda^{ \pm} \int_{\delta^{ \pm}}^{\infty}-k(y) e^{g^{\lambda \pm}}+k(y) e^{g^{\lambda \pm}}+k(y) \log \left(e^{\bar{g}^{ \pm}}+\epsilon k(y)\right) e^{g^{\lambda \pm}} F^{ \pm}(y) d y\right] \\
& =\quad \lambda^{ \pm}\left[\int_{\delta^{ \pm}}^{\infty} e^{g^{\lambda \pm}} k(y) F(y) d y\right]\left(\delta^{ \pm}+\Delta^{ \pm}\right) \\
& +\frac{1}{\varphi_{\kappa}}\left[\lambda^{ \pm} \int_{0}^{\delta^{ \pm}} k(y) \log \left(e^{g^{ \pm}}+\epsilon k(y)\right) e^{g^{\lambda \pm}} F^{ \pm}(y) d y\right] \\
& +\frac{1}{\varphi_{\kappa}}\left[\lambda^{ \pm} \int_{\delta^{ \pm}}^{\infty} k(y) \log \left(e^{\bar{g}^{ \pm}}+\epsilon k(y)\right) e^{g^{\lambda \pm}} F^{ \pm}(y) d y\right]
\end{aligned}
$$

Evaluating this expression at $\epsilon=0$ gives:

$$
\begin{aligned}
& \lambda^{ \pm}\left[\int_{\delta^{ \pm}}^{\infty} e^{g^{\lambda \pm}} k(y) F(y) d y\right]\left(\delta^{ \pm}+\Delta^{ \pm}\right) \\
& +\frac{1}{\varphi_{\kappa}}\left[\lambda^{ \pm} \int_{0}^{\delta^{ \pm}} k(y) \underline{g}^{ \pm} e^{g^{\lambda \pm}} F^{ \pm}(y) d y\right]+\frac{1}{\varphi_{\kappa}}\left[\lambda^{ \pm} \int_{\delta^{ \pm}}^{\infty} k(y) \bar{g}^{ \pm} e^{g^{\lambda \pm}} F^{ \pm}(y) d y\right] \\
= & \lambda^{ \pm}\left[\int_{\delta^{ \pm}}^{\infty} e^{g^{\lambda \pm}} k(y) F(y) d y\right]\left(\delta^{ \pm}+\Delta^{ \pm}\right)-\frac{1}{\varphi_{\kappa}} \lambda^{ \pm} \int_{0}^{\delta^{ \pm}} k(y) \log \left(A^{ \pm}\right) e^{g^{\lambda} \pm} F(y) d y \\
& -\frac{1}{\varphi_{\kappa}} \lambda^{ \pm} \int_{0}^{\delta^{ \pm}} k(y)\left(\log \left(A^{ \pm}\right)+\varphi_{\kappa}\left(\delta^{ \pm}+\Delta^{ \pm}\right)\right) e^{g^{\lambda}} F(y) d y \\
= & 0
\end{aligned}
$$

as expected. Continuing by taking a second derivative with respect to $\epsilon$ :

$$
\begin{aligned}
& \frac{1}{\varphi_{\kappa}} \lambda^{ \pm} \int_{0}^{\delta^{ \pm}} \frac{e^{g^{\lambda \pm}} k^{2}(y)}{e^{g^{ \pm}}+\epsilon k(y)} F(y) d y+\frac{1}{\varphi_{\kappa}} \lambda^{ \pm} \int_{\delta^{ \pm}}^{\infty} \frac{e^{g^{\lambda \pm}} k^{2}(y)}{e^{\bar{g}^{ \pm}}+\epsilon k(y)} F(y) d y \\
= & \frac{1}{\varphi_{\kappa}} \lambda^{ \pm} \int_{0}^{\infty} \frac{e^{g^{\lambda \pm}} k^{2}(y)}{e^{g^{ \pm \kappa}(y)}+\epsilon k(y)} F(y) d y \\
= & \frac{1}{\varphi_{\kappa}} \lambda^{ \pm} \int_{0}^{\infty} \frac{e^{g^{\lambda \pm}} k^{2}(y)}{e^{f_{\epsilon}(y)}} F(y) d y
\end{aligned}
$$

This expression is positive for all $\epsilon \in[0,1]$ showing that indeed the expression for $g^{ \pm \kappa}(y)$ in equation (B.13) is a minimizer. 
Solving for $g^{ \pm \lambda}$ : Substituting the expression (B.13) into the term to be minimized (B.9) and performing some tedious computations gives the term which must be minimized with respect to $g^{ \pm \lambda}$ :

$$
\begin{gathered}
\lambda^{ \pm} e^{g^{ \pm \lambda}} e^{\bar{g}^{ \pm}}\left(\delta^{ \pm}+\Delta^{ \pm}\right) e^{-\kappa^{ \pm} \delta^{ \pm}} \\
+\frac{\lambda^{ \pm}}{\varphi_{\lambda}}\left(-\left(e^{g^{ \pm \lambda}}-1\right)+g^{ \pm \lambda} e^{g^{ \pm \lambda}} e^{\bar{g}^{ \pm}}\right) e^{-\kappa^{ \pm} \delta^{ \pm}}+\frac{\lambda^{ \pm}}{\varphi_{\kappa}}\left(-\left(e^{\bar{g}^{ \pm}}-1\right) e^{g^{ \pm \lambda}}+\bar{g}^{ \pm} e^{g^{ \pm \lambda}} e^{\bar{g}^{ \pm}}\right) e^{-\kappa^{ \pm} \delta^{ \pm}} \\
+\frac{\lambda^{ \pm}}{\varphi_{\lambda}}\left(-\left(e^{g^{ \pm \lambda}}-1\right)+g^{ \pm \lambda} e^{g^{ \pm \lambda}} e^{g^{ \pm}}\right)\left(1-e^{-\kappa^{ \pm} \delta^{ \pm}}\right)+\frac{\lambda^{ \pm}}{\varphi_{\kappa}}\left(-\left(e^{g^{ \pm}}-1\right) e^{g^{ \pm \lambda}}+\underline{g}^{ \pm} e^{g^{ \pm \lambda}} e^{g^{ \pm}}\right)\left(1-e^{-\kappa^{ \pm} \delta^{ \pm}}\right)
\end{gathered}
$$

Applying first order conditions in $g^{ \pm \lambda}$ to this term and doing some tedious computations gives the expression:

$$
g^{ \pm \lambda}=\frac{\varphi_{\lambda}}{\varphi_{\kappa}} \log A^{ \pm}
$$

Solving for $\delta^{ \pm}$: Substituting expressions (B.14) to (B.17) and (B.19) into (B.18) and performing more tedious computation results in the term which must be maximized in terms of $\delta^{ \pm}$:

$$
\frac{\lambda^{ \pm}}{\varphi_{\lambda}}\left(1-\exp \left\{\frac{\varphi_{\lambda}}{\varphi_{\kappa}} \log \left(1-e^{-\kappa^{ \pm} \delta^{ \pm}}+e^{-\varphi_{\kappa}\left(\delta^{ \pm}+h_{q \mp 1}^{\varphi_{\kappa}}-h_{q}^{\varphi_{\kappa}}\right)} e^{-\kappa^{ \pm} \delta^{ \pm}}\right)\right\}\right)
$$

Maximizing this term is equivalent to minimizing:

$$
\exp \left\{\frac{\varphi_{\lambda}}{\varphi_{\kappa}} \log \left(1-e^{-\kappa^{ \pm} \delta^{ \pm}}+e^{-\varphi_{\kappa}\left(\delta^{ \pm}+h_{q \mp 1}^{\varphi_{\kappa}}-h_{q}^{\varphi_{\kappa}}\right)} e^{-\kappa^{ \pm} \delta^{ \pm}}\right)\right\}
$$

which is equivalent to minimizing:

$$
1-e^{-\kappa^{ \pm} \delta^{ \pm}}+e^{-\varphi_{\kappa}\left(\delta^{ \pm}+h_{q \mp 1}^{\varphi_{\kappa}}-h_{q}^{\varphi_{\kappa}}\right)} e^{-\kappa^{ \pm} \delta^{ \pm}}
$$

This is the same minimization that took place in the proof of Proposition 3, and so the form of the optimal $\delta^{ \pm}$is:

$$
\delta^{ \pm}=\left(\frac{1}{\varphi_{\kappa}} \log \left(1+\frac{\varphi_{\kappa}}{\kappa^{ \pm}}\right)-h_{q \mp 1}^{\varphi_{\kappa}}+h_{q}^{\varphi_{\kappa}}\right)_{+}
$$

as desired.

Proof of Proposition 8. Defining $\omega_{q}^{\psi}(t)=e^{\kappa h_{q}^{\psi}(t)}$, or equivalently $h_{q}^{\psi}(t)=\frac{\omega_{q}^{\psi}(t)}{\kappa}$ and 
substituting this into equation (2.27) gives:

$$
\begin{array}{r}
\frac{\partial_{t} \omega_{q}^{\psi}}{\kappa \omega_{q}^{\psi}}+\alpha q-\frac{1}{2} \varphi_{\alpha} \sigma^{2} q^{2}+\frac{\xi^{+}}{\kappa} \frac{\omega_{q-1}^{\psi}}{\omega_{q}^{\psi}} \mathbb{1}_{q \neq \underline{q}}+\frac{\xi^{-}}{\kappa} \frac{\omega_{q+1}^{\psi}}{\omega_{q}^{\psi}} \mathbb{1}_{q \neq \bar{q}}=0 \\
\partial_{t} \omega_{q}^{\psi}+\left(\alpha \kappa q-\frac{1}{2} \varphi_{\alpha} \kappa \sigma^{2} q^{2}\right) \omega_{q}^{\psi}+\xi^{+} \omega_{q-1}^{\psi} \mathbb{1}_{q \neq \underline{q}}+\xi^{-} \omega_{q+1}^{\psi} \mathbb{1}_{q \neq \bar{q}}=0,
\end{array}
$$

together with terminal conditions $\omega_{q}^{\psi}(T)=e^{-\kappa q \ell(q)}$. With the matrix $\mathbf{A}^{\psi}$ defined as in equation (2.28), the system of ODE's for $\omega_{q}^{\psi}$ can be written as:

$$
\partial_{t} \boldsymbol{\omega}^{\psi}+\mathbf{A}^{\psi} \boldsymbol{\omega}^{\psi}=0
$$

This system of equations has solution

$$
\boldsymbol{\omega}^{\psi}(t)=e^{\mathbf{A}^{\psi}(T-t)} \boldsymbol{\omega}^{\psi}(T)
$$

as desired.

\section{B.1.1 Verification Theorem}

Proof of Proposition 10. The proof is given for equation (2.24) as it is the most general and the others follow along the same lines. The equation of interest is:

$$
\begin{gathered}
\partial_{t} h_{q}^{\psi}+\alpha q-\frac{1}{2} \varphi_{\alpha} \sigma^{2} q^{2} \\
+\sup _{\delta^{+} \geq 0}\left\{\frac{\lambda^{+}}{\varphi_{\lambda}}\left(1-\exp \left\{\frac{\varphi_{\lambda}}{\varphi_{\kappa}} \log \left(1-e^{-\kappa^{+} \delta^{+}}+e^{-\kappa^{+} \delta^{+}-\varphi_{\kappa}\left(\delta^{+}+h_{q-1}^{\psi}-h_{q}^{\psi}\right)}\right)\right\}\right)\right\} \mathbb{1}_{q \neq \underline{q}} \\
+\sup _{\delta^{-} \geq 0}\left\{\frac{\lambda^{-}}{\varphi_{\lambda}}\left(1-\exp \left\{\frac{\varphi_{\lambda}}{\varphi_{\kappa}} \log \left(1-e^{-\kappa^{-} \delta^{-}}+e^{-\kappa^{-} \delta^{-}-\varphi_{\kappa}\left(\delta^{-}+h_{q+1}^{\psi}-h_{q}^{\psi}\right)}\right)\right\}\right)\right\} \mathbb{1}_{q \neq \bar{q}}=0, \\
h_{q}^{\psi}(T)=-q \ell(q) .
\end{gathered}
$$

To show existence and uniqueness of the solution to this equation, the function

$$
f(x, y)=\sup _{\delta \geq 0}\left\{\frac{\lambda}{\varphi_{\lambda}}\left(1-\exp \left\{\frac{\varphi_{\lambda}}{\varphi_{\kappa}} \log \left(1-e^{-\kappa \delta}+e^{-\kappa \delta-\varphi_{\kappa}(\delta+x-y)}\right)\right\}\right)\right\}
$$

is shown to be globally Lipschitz. Writing the equation as $\partial_{t} h_{q}^{\psi}=F\left(q, \boldsymbol{h}^{\psi}\right)$, the global Lipschitz property of $f$ will then imply the global Lipschitz property (with respect to $\boldsymbol{h}^{\psi}$ ) for $F$, and thus the desired result is achieved. 
The supremum is attained at $\delta=\left(\frac{1}{\varphi_{\kappa}} \log \left(1+\frac{\varphi_{\kappa}}{\kappa}\right)-x+y\right)_{+}$as shown in the proof of Proposition 7. Thus, two separate domains for $f$ must be considered: $\frac{1}{\varphi_{\kappa}} \log \left(1+\frac{\varphi_{\kappa}}{\kappa}\right)>$ $x-y$ and $\frac{1}{\varphi_{\kappa}} \log \left(1+\frac{\varphi_{\kappa}}{\kappa}\right) \leq x-y$. Substituting the optimal $\delta$ into the expression for $f$ yields:

$$
f(x, y)=\frac{\lambda}{\varphi_{\lambda}}\left(1-e^{-\varphi_{\lambda}(x-y)}\right)
$$

when $\frac{1}{\varphi_{\kappa}} \log \left(1+\frac{\varphi_{\kappa}}{\kappa}\right) \leq x-y$, or

$$
f(x, y)=\frac{\lambda}{\varphi_{\lambda}}\left(1-\exp \left\{\frac{\varphi_{\lambda}}{\varphi_{\kappa}} \log \left(1-e^{-\frac{\kappa}{\varphi_{\kappa}} \log \left(1+\frac{\varphi_{\kappa}}{\kappa}\right)+\kappa(x-y)}\left(1-e^{-\log \left(1+\frac{\varphi_{\kappa}}{\kappa}\right)}\right)\right)\right\}\right)
$$

when $\frac{1}{\varphi_{\kappa}} \log \left(1+\frac{\varphi_{\kappa}}{\kappa}\right)>x-y$. Some simplifications can be made for the second expression:

$$
\begin{aligned}
& \frac{\lambda}{\varphi_{\lambda}}\left(1-\exp \left\{\frac{\varphi_{\lambda}}{\varphi_{\kappa}} \log \left(1-e^{-\frac{\kappa}{\varphi_{\kappa}} \log \left(1+\frac{\varphi_{\kappa}}{\kappa}\right)+\kappa(x-y)}\left(1-e^{-\log \left(1+\frac{\varphi_{\kappa}}{\kappa}\right)}\right)\right)\right\}\right) \\
= & \frac{\lambda}{\varphi_{\lambda}}\left(1-\exp \left\{\frac{\varphi_{\lambda}}{\varphi_{\kappa}} \log \left(1-\left(\frac{\kappa}{\varphi_{\kappa}+\kappa}\right)^{\frac{\kappa}{\varphi_{\kappa}}} \frac{\varphi_{\kappa}}{\varphi_{\kappa}+\kappa} e^{\kappa(x-y)}\right)\right\}\right) \\
= & \frac{\lambda}{\varphi_{\lambda}}\left(1-\exp \left\{\frac{\varphi_{\lambda}}{\varphi_{\kappa}} \log \left(1-B e^{\kappa(x-y)}\right)\right\}\right)
\end{aligned}
$$

where $B=\left(\frac{\kappa}{\varphi_{\kappa}+\kappa}\right)^{\frac{\kappa}{\varphi_{\kappa}}} \frac{\varphi_{\kappa}}{\varphi_{\kappa}+\kappa}>0$. The global Lipschitz property will be shown for $f$ by showing that it's one-sided partial derivatives exist everywhere and are bounded. Consider first the domain $\frac{1}{\varphi_{\kappa}} \log \left(1+\frac{\varphi_{\kappa}}{\kappa}\right) \leq x-y$ :

$$
\begin{aligned}
f(x, y) & =\frac{\lambda}{\varphi_{\lambda}}\left(1-e^{-\varphi_{\lambda}(x-y)}\right) \\
\partial_{x} f(x, y) & =\lambda e^{-\varphi_{\lambda}(x-y)} \\
\left|\partial_{x} f(x, y)\right| & \leq \lambda e^{-\frac{\varphi_{\lambda}}{\varphi_{\kappa}} \log \left(1+\frac{\varphi_{\kappa}}{\kappa}\right)} \\
\partial_{y} f(x, y) & =-\lambda e^{-\varphi_{\lambda}(x-y)} \\
\left|\partial_{y} f(x, y)\right| & \leq \lambda e^{-\frac{\varphi_{\lambda}}{\varphi_{\kappa}} \log \left(1+\frac{\varphi_{\kappa}}{\kappa}\right)}
\end{aligned}
$$

Thus, in the interior of the domain, the partial derivatives exist and are bounded by $\lambda e^{-\frac{\varphi_{\lambda}}{\varphi_{\kappa}} \log \left(1+\frac{\varphi_{\kappa}}{\kappa}\right)}$. On the boundary of the domain, the one sided partial derivatives into the domain exist and have the same bound. 
For the second domain, $\frac{1}{\varphi_{\kappa}} \log \left(1+\frac{\varphi_{\kappa}}{\kappa}\right)>x-y$ :

$$
\begin{aligned}
f(x, y) & =\frac{\lambda}{\varphi_{\lambda}}\left(1-e^{\frac{\varphi_{\lambda}}{\varphi_{\kappa}} \log \left(1-B e^{\kappa(x-y)}\right)}\right) \\
\partial_{x} f(x, y) & =\frac{\lambda}{\varphi_{\kappa}} e^{\frac{\varphi_{\lambda}}{\varphi_{\kappa}} \log \left(1-B e^{\kappa(x-y)}\right)} \frac{B \kappa e^{\kappa(x-y)}}{1-B e^{\kappa(x-y)}}
\end{aligned}
$$

Letting $z=B e^{\kappa(x-y)}$, the domain of consideration implies:

$$
\begin{aligned}
z & <\left(\frac{\kappa}{\varphi_{\kappa}+\kappa}\right)^{\frac{\kappa}{\varphi_{\kappa}}} \frac{\varphi_{\kappa}}{\varphi_{\kappa}+\kappa} e^{\frac{\kappa}{\varphi_{\kappa}} \log \left(1+\frac{\varphi_{\kappa}}{\kappa}\right)} \\
& =\left(\frac{\kappa}{\varphi_{\kappa}+\kappa}\right)^{\frac{\kappa}{\varphi_{\kappa}}} \frac{\varphi_{\kappa}}{\varphi_{\kappa}+\kappa}\left(\frac{\varphi_{\kappa}+\kappa}{\kappa}\right)^{\frac{\kappa}{\varphi_{\kappa}}} \\
& =\frac{\varphi_{\kappa}}{\varphi_{\kappa}+\kappa} \\
& <1
\end{aligned}
$$

And clearly $z$ is also bounded below by 0 . Continuing with the partial derivatives:

$$
\partial_{x} f(x, y)=\frac{\lambda}{\varphi_{\kappa}} e^{\frac{\varphi_{\lambda}}{\varphi_{\kappa}} \log (1-z)} \frac{z}{1-z}
$$

This expression is non-negative and continuous for $0 \leq z \leq \frac{\varphi_{\kappa}}{\varphi_{\kappa}+\kappa}$, and so it achieves a finite maximum somewhere on that interval. Thus, $\partial_{x} f(x, y)$ is bounded. Similarly:

$$
\begin{aligned}
& \partial_{y} f(x, y)=\frac{\lambda}{\varphi_{\lambda}}\left(1-e^{\frac{\varphi_{\lambda}}{\varphi_{\kappa}} \log \left(1-B e^{\kappa(x-y)}\right)}\right) \\
& \partial_{y} f(x, y)=-\frac{\lambda}{\varphi_{\kappa}} e^{\frac{\varphi_{\lambda}}{\varphi_{\kappa}} \log \left(1-B e^{\kappa(x-y)}\right)} \frac{B \kappa e^{\kappa(x-y)}}{1-B e^{\kappa(x-y)}}
\end{aligned}
$$

which is also bounded, by the same bound as for $\partial_{x} f(x, y)$. In the interior of this domain, both partial derivatives exist and are bounded. On the boundary, the one-sided partial derivatives into the interior of the domain exist with the same bounds. With both one-sided partial derivatives existing and being bounded in both domains, the Lipschitz 
condition is shown easily:

$$
\begin{aligned}
\left|f\left(x_{2}, y_{2}\right)-f\left(x_{1}, y_{1}\right)\right| & =\left|\int_{C} \nabla f(x, y) \cdot d \vec{r}\right| \\
& \leq \int_{C}|\nabla f(x, y)| d s \\
& \leq \int_{C} M d s \\
& =M\left|\left(x_{2}, y_{2}\right)-\left(x_{1}, y_{1}\right)\right|
\end{aligned}
$$

where $C$ is the curve which connects $\left(x_{1}, y_{1}\right)$ to $\left(x_{2}, y_{2}\right)$ in a straight line. Since $f$ is Lipschitz, it is also true that $F$ is Lipschitz, where $\partial_{t} h_{q}^{\psi}=F\left(q, \boldsymbol{h}^{\psi}\right)$. Finally, it is shown that $f$ is bounded: for $x-y \geq \frac{1}{\varphi_{\kappa}} \log \left(1+\frac{\varphi_{\kappa}}{\kappa}\right)$ :

$$
\begin{aligned}
|f(x, y)| & =\frac{\lambda}{\varphi_{\lambda}}\left(1-e^{-\varphi_{\lambda}(x-y)}\right) \\
& \leq \frac{\lambda}{\varphi_{\lambda}}\left(1-e^{-\frac{\varphi_{\lambda}}{\varphi_{\kappa}} \log \left(1+\frac{\varphi_{\kappa}}{\kappa}\right)}\right)
\end{aligned}
$$

and for $x-y<\frac{1}{\varphi_{\kappa}} \log \left(1+\frac{\varphi_{\kappa}}{\kappa}\right)$ :

$$
f(x, y)=\frac{\lambda}{\varphi_{\lambda}}\left(1-e^{\frac{\varphi_{\lambda}}{\varphi_{\kappa}} \log \left(1-B e^{\kappa(x-y)}\right)}\right)
$$

Making the substitution $z=B e^{\kappa(x, y)}$ again, with $0<z \leq \frac{\varphi_{\kappa}}{\varphi_{\kappa}+\kappa}$, it is easy to see that the expression

$$
f(x, y)=\frac{\lambda}{\varphi_{\lambda}}\left(1-e^{\frac{\varphi_{\lambda}}{\varphi_{\kappa}} \log (1-z)}\right)
$$

is bounded. Boundedness of $f$ implies boundedness of $F$, then by an application of the Picard-Lindelöf theorem, $\boldsymbol{h}^{\psi}$ has a unique solution.

Proof of Theorem 11. Let $\hat{H}^{\psi}(t, x, S, q)=x+q S+h_{q}^{\psi}(t)$ be a candidate function for the solution to the optimal control problem, where $h_{q}^{\psi}(t)$ satisfies equation (2.29) with 
boundary conditions $h_{q}^{\psi}(T)=-q \ell(q)$. Then from Ito's lemma:

$$
\begin{aligned}
\hat{H}^{\alpha, \lambda, \kappa}\left(T, X_{T^{-}}^{\delta^{ \pm}}, S_{T^{-}}, q_{T^{-}}^{\delta^{ \pm}} ; \psi\right)= & \hat{H}^{\alpha, \lambda, \kappa}(t, x, S, q ; \psi)+\int_{t}^{T} \partial_{t} h_{q_{s}}^{\psi}(s) d s \\
& +\int_{t}^{T} \alpha q_{s}^{\delta^{ \pm}} d s+\sigma \int_{t}^{T} q_{s}^{\delta^{ \pm}} d W_{s} \\
& +\int_{t}^{T} \int_{\delta_{s}^{+}}^{\infty}\left(\delta_{s}^{+}+h_{q_{s}-1}^{\psi}(s)-h_{q_{s}}^{\psi}(s)\right) \mu^{+}(d y, d s) \\
& +\int_{t}^{T} \int_{\delta_{s}^{-}}^{\infty}\left(\delta_{s}^{-}+h_{q_{s}+1}^{\psi}(s)-h_{q_{s}}^{\psi}(s)\right) \mu^{-}(d y, d s) .
\end{aligned}
$$

Let $\eta_{t}^{*}, g_{t}^{ \pm \lambda *}$, and $g_{t}^{ \pm \kappa *}(y)$ be the optimal controls resulting from Proposition 7 , and let $\delta_{t}^{ \pm}$be an arbitrary admissible control. From equation (2.29) and Proposition 10:

$$
\begin{aligned}
& \mathbb{E}_{t, x, q, S}^{\mathbb{Q}^{\alpha, \lambda, \kappa}\left(\eta^{*}, g^{*}\right)}\left[\hat{H}^{\alpha, \lambda, \kappa}\left(T, X_{T^{-}}^{\delta^{ \pm}}, S_{T^{-}}, q_{T^{-}}^{\delta^{ \pm}} ; \psi\right)\right] \\
& =\hat{H}^{\alpha, \lambda, \kappa}(t, x, S, q ; \psi)+\mathbb{E}_{t, x, q, S}^{\mathbb{Q}^{\alpha, \lambda, \kappa}\left(\eta^{*}, g^{*}\right)}\left[\int_{t}^{T} \partial_{t} h_{q_{s}}^{\psi}(s) d s+\int_{t}^{T} \alpha q_{s}^{\delta^{ \pm}} d s+\sigma \int_{t}^{T} q_{s}^{\delta^{ \pm}} d W_{s}\right. \\
& +\int_{t}^{T} \int_{\delta_{s}^{+}}^{\infty}\left(\delta_{s}^{+}+h_{q_{s}-1}^{\psi}(s)-h_{q_{s}}^{\psi}(s)\right) \nu_{\mathbb{Q}^{\alpha, \lambda, \kappa}\left(\eta^{*}, g^{*}\right)}^{+}(d y, d s) \\
& \left.+\int_{t}^{T} \int_{\delta_{s}^{-}}^{\infty}\left(\delta_{s}^{-}+h_{q_{s}+1}^{\psi}(s)-h_{q_{s}}^{\psi}(s)\right) \nu_{\mathbb{Q}^{\alpha, \lambda, \kappa}\left(\eta^{*}, g^{*}\right)}^{-}(d y, d s)\right] \\
& \leq \hat{H}^{\alpha, \lambda, \kappa}(t, x, S, q ; \psi)+\mathbb{E}_{t, x, q, S}^{\mathbb{Q}^{\alpha, \lambda, \kappa}\left(\eta^{*}, g^{*}\right)}\left[-\frac{1}{2 \varphi_{\alpha}} \int_{t}^{T}\left(\frac{\alpha-\eta_{s}^{*}}{\sigma}\right)^{2} d s\right. \\
& -\int_{t}^{T}\left(\mathcal{K}_{+}^{\varphi_{\lambda}, \varphi_{\kappa}}\left(g_{s}^{+\lambda *}, g_{s}^{+\kappa *}\right) \mathbb{1}_{\varphi_{\lambda} \geq \varphi_{\kappa}}+\mathcal{K}_{+}^{\varphi_{\kappa}, \varphi_{\lambda}}\left(g_{s}^{+\kappa *}, g_{s}^{+\lambda *}\right) \mathbb{1}_{\varphi_{\lambda}<\varphi_{\kappa}}\right) d s \\
& \left.-\int_{t}^{T}\left(\mathcal{K}_{-}^{\varphi_{\lambda}, \varphi_{\kappa}}\left(g_{s}^{-\lambda *}, g_{s}^{-\kappa *}\right) \mathbb{1}_{\varphi_{\lambda} \geq \varphi_{\kappa}}+\mathcal{K}_{-}^{\varphi_{\kappa}, \varphi_{\lambda}}\left(g_{s}^{-\kappa *}, g_{s}^{-\lambda *}\right) \mathbb{1}_{\varphi_{\lambda}<\varphi_{\kappa}}\right) d s\right] .
\end{aligned}
$$


And so the candidate function satisfies

$$
\begin{aligned}
\hat{H}^{\alpha, \lambda, \kappa}(t, x, S, q ; \psi) \geq & \mathbb{E}_{t, x, q, S}^{\mathbb{Q}^{\alpha, \lambda, \kappa}\left(\eta^{*}, g^{*}\right)}\left[\hat{H}^{\alpha, \lambda, \kappa}\left(T, X_{T^{-}}^{\delta^{ \pm}}, S_{T^{-}}, q_{T^{-}}^{\delta^{ \pm}} ; \psi\right)+\frac{1}{2 \varphi_{\alpha}} \int_{t}^{T}\left(\frac{\alpha-\eta_{s}^{*}}{\sigma}\right)^{2} d s\right. \\
& \left.+\sum_{ \pm} \int_{t}^{T}\left(\mathcal{K}_{ \pm}^{\varphi_{\lambda}, \varphi_{\kappa}}\left(g_{s}^{ \pm \lambda *}, g_{s}^{ \pm \kappa *}\right) \mathbb{1}_{\varphi_{\lambda} \geq \varphi_{\kappa}}+\mathcal{K}_{ \pm}^{\varphi_{\kappa}, \varphi_{\lambda}}\left(g_{s}^{ \pm \kappa *}, g_{s}^{ \pm \lambda *}\right) \mathbb{1}_{\varphi_{\lambda}<\varphi_{\kappa}}\right) d s\right] \\
= & \mathbb{E}_{t, x, q, S}^{\mathbb{Q}^{\alpha, \lambda, \kappa}\left(\eta^{*}, g^{*}\right)}\left[\hat{H}^{\alpha, \lambda, \kappa}\left(T, X_{T}^{\delta^{ \pm}}, S_{T}, q_{T}^{\delta^{ \pm}} ; \psi\right)+\mathcal{H}_{t, T}\left(\mathbb{Q}^{\alpha, \lambda, \kappa}\left(\eta^{*}, g^{*}\right) \mid \mathbb{P}\right)\right] \\
= & \mathbb{E}_{t, x, q, S}^{\mathbb{Q}^{\alpha, \lambda, \kappa}\left(\eta^{*}, g^{*}\right)}\left[X_{T}^{\delta^{ \pm}}+q_{T}^{\delta^{ \pm}}\left(S_{T}-\ell\left(q_{T}^{\delta^{ \pm}}\right)\right)+\mathcal{H}_{t, T}\left(\mathbb{Q}^{\alpha, \lambda, \kappa}\left(\eta^{*}, g^{*}\right) \mid \mathbb{P}\right)\right] \\
\hat{H}^{\alpha, \lambda, \kappa}(t, x, S, q ; \psi) \geq & \sup _{\left(\delta_{s}^{ \pm}\right)_{t \leq s \leq T} \in \mathcal{A}} \mathbb{E}_{t, x, q, S}^{\mathbb{Q}^{\alpha, \lambda, \kappa}\left(\eta^{*}, g^{*}\right)}\left[X_{T}^{\delta_{s}^{ \pm}}+q_{T}^{\delta^{ \pm}}\left(S_{T}-\ell\left(q_{T}^{\delta^{ \pm}}\right)\right)+\mathcal{H}_{t, T}\left(\mathbb{Q}^{\alpha, \lambda, \kappa}\left(\eta^{*}, g^{*}\right) \mid \mathbb{P}\right)\right] \\
\hat{H}^{\alpha, \lambda, \kappa}(t, x, S, q ; \psi) \geq & \sup _{\left(\delta_{s}^{ \pm}\right)_{t \leq s \leq T} \in \mathcal{A}} \inf _{\mathbb{Q} \in \mathcal{Q}^{\alpha, \lambda, \kappa}} \mathbb{E}_{t, x, q, S}^{\mathbb{Q}}\left[X_{T}^{\delta_{s}^{ \pm}}+q_{T}^{\delta^{ \pm}}\left(S_{T}-\ell\left(q_{T}^{\delta^{ \pm}}\right)\right)+\mathcal{H}_{t, T}(\mathbb{Q} \mid \mathbb{P})\right] \\
= & H^{\alpha, \lambda, \kappa}(t, x, S, q ; \psi) .
\end{aligned}
$$

Now, let $\delta_{t}^{ \pm *}$ be the optimal control that corresponds to Proposition 7, and let $\eta_{t}, g_{t}^{ \pm \lambda}$ and $g_{t}^{ \pm \kappa}(y)$ be arbitrary such that they induce an admissible measure $\mathbb{Q}^{\alpha, \lambda, \kappa}(\eta, g) \in \mathcal{Q}^{\alpha, \lambda, \kappa}$. Then from Ito's lemma and equation (2.29) and Proposition 10 again:

$$
\begin{gathered}
\mathbb{E}_{t, x, q, S}^{\mathbb{Q}^{\alpha, \lambda, \kappa}(\eta, g)}\left[\hat{H}^{\alpha, \lambda, \kappa}\left(T, X_{T^{-}}^{\delta^{ \pm *}}, S_{T^{-}}, q_{T^{-}}^{\delta^{ \pm *}} ; \psi\right)\right] \\
=\hat{H}^{\alpha, \lambda, \kappa}(t, x, S, q ; \psi)+\mathbb{E}_{t, x, q, S}^{\mathbb{Q}^{\alpha, \lambda, \kappa}(\eta, g)}\left[\int_{t}^{T} \partial_{t} h_{q_{s}}^{\psi}(s) d s+\int_{t}^{T} \alpha q_{s}^{\delta^{ \pm *}} d s+\sigma \int_{t}^{T} q_{s}^{\delta^{ \pm *}} d W_{s}\right. \\
\left.+\sum_{ \pm} \int_{t}^{T} \int_{\delta_{s}^{ \pm *}}^{\infty}\left(\delta_{s}^{ \pm *}+h_{q_{s} \mp 1}^{\psi}(s)-h_{q_{s}}^{\psi}(s)\right) \nu_{\mathbb{Q}^{\alpha, \lambda, \kappa}(\eta, g)}^{ \pm}(d y, d s)\right] \\
\geq \hat{H}^{\alpha, \lambda, \kappa}(t, x, S, q ; \psi)+\mathbb{E}_{t, x, q, S}^{\mathbb{Q}^{\alpha, \lambda, \kappa}(\eta, g)}\left[-\frac{1}{2 \varphi_{\alpha}} \int_{t}^{T}\left(\frac{\alpha-\eta_{s}}{\sigma}\right)^{2} d s\right. \\
\left.-\sum_{ \pm} \int_{t}^{T}\left(\mathcal{K}_{ \pm}^{\varphi_{\lambda}, \varphi_{\kappa}}\left(g_{s}^{ \pm \lambda}, g_{s}^{ \pm \kappa}\right) \mathbb{1}_{\varphi_{\lambda} \geq \varphi_{\kappa}}+\mathcal{K}_{ \pm}^{\varphi_{\kappa}, \varphi_{\lambda}}\left(g_{s}^{ \pm \kappa}, g_{s}^{ \pm \lambda}\right) \mathbb{1}_{\varphi_{\lambda}<\varphi_{\kappa}}\right) d s\right] .
\end{gathered}
$$


And so the candidate function satisfies

$$
\begin{aligned}
& \hat{H}^{\alpha, \lambda, \kappa}(t, x, S, q ; \psi) \leq \mathbb{E}_{t, x, q, S}^{\mathbb{Q}^{\alpha, \lambda, \kappa}(\eta, g)}\left[\hat{H}^{\alpha, \lambda, \kappa}\left(T, X_{T^{-}}^{\delta^{ \pm *}}, S_{T^{-}}, q_{T^{-}}^{\delta^{ \pm *}} ; \psi\right)+\frac{1}{2 \varphi_{\alpha}} \int_{t}^{T}\left(\frac{\alpha-\eta_{s}}{\sigma}\right)^{2} d s\right. \\
&\left.+\sum_{ \pm} \int_{t}^{T}\left(\mathcal{K}_{ \pm}^{\varphi_{\lambda}, \varphi_{\kappa}}\left(g_{s}^{ \pm \lambda}, g_{s}^{ \pm \kappa}\right) \mathbb{1}_{\varphi_{\lambda} \geq \varphi_{\kappa}}+\mathcal{K}_{ \pm}^{\varphi_{\kappa}, \varphi_{\lambda}}\left(g_{s}^{ \pm \kappa}, g_{s}^{ \pm \lambda}\right) \mathbb{1}_{\varphi_{\lambda}<\varphi_{\kappa}}\right) d s\right] \\
&= \mathbb{E}_{t, x, q, S}^{\mathbb{Q}^{\alpha, \lambda, \kappa}(\eta, g)}\left[\hat{H}^{\alpha, \lambda, \kappa}\left(T, X_{T}^{\delta^{ \pm *}}, S_{T}, q_{T}^{\delta^{ \pm *}} ; \psi\right)+\mathcal{H}_{t, T}\left(\mathbb{Q}^{\alpha, \lambda, \kappa}(\eta, g) \mid \mathbb{P}\right)\right] \\
&= \mathbb{E}_{t, x, q, S}^{\mathbb{Q}^{\alpha, \lambda, \kappa}(\eta, g)}\left[X_{T}^{\delta^{ \pm *}}+q_{T}^{\delta^{ \pm *}}\left(S_{T}-\ell\left(q_{T}^{\delta^{ \pm *}}\right)\right)+\mathcal{H}_{t, T}\left(\mathbb{Q}^{\alpha, \lambda, \kappa}(\eta, g) \mid \mathbb{P}\right)\right] \\
& \hat{H}^{\alpha, \lambda, \kappa}(t, x, S, q ; \psi) \leq \inf _{\mathbb{Q} \in \mathcal{Q}^{\alpha, \lambda, \kappa}} \mathbb{E}_{t, x, q, S}^{\mathbb{Q}}\left[X_{T}^{\delta_{s}^{ \pm *}}+q_{T}^{\delta^{ \pm *}}\left(S_{T}-\ell\left(q_{T}^{\delta^{ \pm *}}\right)\right)+\mathcal{H}_{t, T}(\mathbb{Q} \mid \mathbb{P})\right] \\
& \hat{H}^{\alpha, \lambda, \kappa}(t, x, S, q ; \psi) \leq \sup _{\left(\delta_{s}^{ \pm}\right)_{t \leq s \leq T} \in \mathcal{A}} \inf _{\mathbb{Q} \in \mathcal{Q}^{\alpha, \lambda, \kappa}} \mathbb{E}_{t, x, q, S}^{\mathbb{Q}}\left[X_{T}^{\delta_{s}^{ \pm}}+q_{T}^{\delta^{ \pm}}\left(S_{T}-\ell\left(q_{T}^{\delta^{ \pm}}\right)\right)+\mathcal{H} t, T(\mathbb{Q} \mid \mathbb{P})\right] \\
&= H^{\alpha, \lambda, \kappa}(t, x, S, q ; \psi) .
\end{aligned}
$$

The result follows from Equations (B.20) and (B.21).

\section{B.2 Proofs from Chapter 3}

Proof of Proposition 17. Let $\omega_{q}^{\psi}(t)=e^{\kappa h_{q}^{\psi}(t)}$, or equivalently, $h_{q}^{\psi}(t)=\frac{1}{\kappa} \omega_{q}^{\psi}(t)$. Substituting this into equation (3.18) gives:

$$
\begin{aligned}
\frac{\partial_{t} \omega_{q}^{\psi}}{\kappa \omega_{q}^{\psi}}+\alpha q-\frac{1}{2} \varphi_{\alpha} \sigma^{2} q^{2}+\frac{\xi}{\kappa} \frac{\omega_{q-1}^{\psi}}{\omega_{q}^{\psi}} \mathbb{1}_{q \neq 0} & =0 \\
\partial_{t} \omega_{q}^{\psi}+\alpha \kappa q \omega_{q}^{\psi}-\frac{1}{2} \varphi_{\alpha} \kappa \sigma^{2} q^{2} \omega_{q}^{\psi}+\xi \omega_{q-1}^{\psi} \mathbb{1}_{q \neq 0} & =0 \\
\partial_{t} \omega_{q}^{\psi}+K_{q} \omega_{q}^{\psi}+\xi \omega_{q-1}^{\psi} \mathbb{1}_{q \neq 0} & =0
\end{aligned}
$$

together with terminal conditions $\omega_{q} \psi(T)=e^{-\kappa q \ell(q)}$. To prove part i), equation (B.22) becomes:

$$
\partial_{t} \omega_{q}=-\xi \omega_{q-1}^{\psi} \mathbb{1}_{q \neq 0}
$$


For $q=0$ this results in $\omega_{0}^{\psi}(t)=1$. For $q>0$, integrating both sides yields:

$$
\begin{aligned}
\omega_{q}^{\psi}(T)-\omega_{q} \psi(t) & =-\xi \int_{t}^{T} \omega_{q-1}^{\psi} \\
\omega_{q}^{\psi}(t) & =\xi \int_{t}^{T} \omega_{q-1}^{\psi}(u) d u+\omega_{q}^{\psi}(T)
\end{aligned}
$$

Since $\omega_{0}^{\psi}(t)$ is a constant and each $\omega_{q}^{\psi}$ results from the integral of $\omega_{q-1}^{\psi}, \omega_{q}^{\psi}(t)$ can be written:

$$
\omega_{q}^{\psi}(t)=\sum_{n=0}^{q} C_{q, n}(T-t)^{n}
$$

where each $C_{q, n}$ must be computed. Substituting equation B.24 into equation (B.23) gives:

$$
\begin{aligned}
\omega_{q}^{\psi}(t) & =\xi \int_{t}^{T} \sum_{n=0}^{q-1} C_{q-1, n}(T-u)^{n}+\omega_{q}^{\psi}(T) \\
& =\xi \sum_{n=0}^{q-1} C_{q-1, n} \int_{t}^{T}(T-u)^{n}+\omega_{q}^{\psi}(T) \\
& =\xi \sum_{n=0}^{q-1} C_{q-1, n} \frac{(T-t)^{n+1}}{n+1}+\omega_{q}^{\psi}(T) \\
& =\xi \sum_{n=1}^{q} C_{q-1, n-1} \frac{(T-t)^{n}}{n}+\omega_{q}^{\psi}(T)
\end{aligned}
$$

From here it is easy to see that:

$$
\begin{aligned}
C_{q, n} & =\frac{\xi}{n} C_{q-1, n-1}, \quad \text { and } \\
C_{q, 0} & =\omega_{q}^{\psi}(T)
\end{aligned}
$$

Together, these give the desired result:

$$
C_{q, n}=\frac{\xi^{n}}{n !} \omega_{q-n}^{\psi}(T)=\frac{\xi^{n}}{n !} e^{-\kappa(q-n) \ell(q-n)}
$$


To prove part ii), return to equation (B.22). Since $K_{0}=0$, it is easily seen that $\omega_{0}^{\psi}(t)=1$. For $q>0$, a recursive solution to equation (B.22) can be written:

$$
\begin{aligned}
\partial_{t} \omega_{q}^{\psi}+K_{q} \omega_{q}^{\psi}+\xi \omega_{q-1}^{\psi} & =0 \\
\partial_{t}\left(e^{K_{q} t} \omega_{q}^{\psi}(t)\right) & =-\xi e^{K_{q} t} \omega_{q-1}^{\psi} \\
e^{K_{q} T} \omega_{q}^{\psi}(T)-e^{K_{q} t} \omega_{q}^{\psi}(t) & =-\xi \int_{t}^{T} e^{K_{q} u} \omega_{q-1}^{\psi}(u) d u \\
\omega_{q}^{\psi}(t) & =\xi e^{-K_{q} t} \int_{t}^{T} e^{K_{q} u} \omega_{q-1}^{\psi}(u) d u+\omega_{q}^{\psi}(T) e^{K_{q}(T-t)}
\end{aligned}
$$

With $\omega_{q}^{\psi}(t)=1$ and each $\omega_{q-1}^{\psi}(t)$ being integrated against $e^{K_{q} t}$, the general form of $\omega_{q}^{\psi}(t)$ can be written as:

$$
\omega_{q}^{\psi}(t)=\sum_{n=0}^{q} C_{q, n} e^{K_{n}(T-t)}
$$

where each $C_{q, n}$ must be computed. Substituting equation (B.26) for $\omega_{q-1 \psi}$ into equation (B.25) gives:

$$
\begin{aligned}
\omega_{q}^{\psi}(t) & =\xi e^{-K_{q} t} \int_{t}^{T} e^{K_{q} u} \sum_{n=0}^{q-1} C_{q-1, n} e^{K_{n}(T-u)} d u+\omega_{q}^{\psi}(T) e^{K_{q}(T-t)} \\
& =\xi e^{-K_{q} t} \int_{t}^{T} \sum_{n=0}^{q-1} C_{q-1, n} e^{K_{n} T} e^{\left(K_{q}-K_{n}\right) u} d u+\omega_{q}^{\psi}(T) e^{K_{q}(T-t)} \\
& =\xi e^{-K_{q} t} \sum_{n=0}^{q-1} C_{q-1, n} e^{K_{n} T} \int_{t}^{T} e^{\left(K_{q}-K_{n}\right) u} d u+\omega_{q}^{\psi}(T) e^{K_{q}(T-t)} \\
& =\xi e^{-K_{q} t} \sum_{n=0}^{q-1} C_{q-1, n} e^{K_{n} T} \frac{e^{\left(K_{q}-K_{n}\right) T}-e^{\left(K_{q}-K_{n}\right) t}}{K_{q}-K_{n}}+\omega_{q}^{\psi}(T) e^{K_{q}(T-t)} \\
& =\xi \sum_{n=0}^{q-1} C_{q-1, n} \frac{e^{K_{q}(T-t)}-e^{K_{n}(T-t)}}{K_{q}-K_{n}}+\omega_{q}^{\psi}(T) e^{K_{q}(T-t)} \\
& =-\xi \sum_{n=0}^{q-1} \frac{C_{q-1, n}}{K_{q}-K_{n}} e^{K_{n}(T-t)}+\left(\xi \sum_{n=0}^{q-1} \frac{C_{q-1, n}}{K_{q}-K_{n}}+\omega_{q}^{\psi}(T)\right) e^{K_{q}(T-t)}
\end{aligned}
$$

From the first summation term above, it is deduced that $C_{q, n}=\frac{-\xi C_{q-1, n}}{K_{q}-K_{n}}$ for $q>n$. This recursive relation leads to:

$$
C_{n+j, n}=(-\xi)^{j} \prod_{p=1}^{j} \frac{1}{K_{n+p}-K_{n}} C_{n, n}
$$


The second summation term in equation (B.27) leads to $C_{q, q}=\xi \sum_{n=0}^{q-1} \frac{C_{q-1, n}}{K_{q}-K_{n}}+\omega_{q}^{\psi}(T)$, which from the previous recursion is the same as:

$$
C_{q, q}=-\sum_{n=0}^{q-1} C_{q, n}+\omega_{q}^{\psi}(T) .
$$

These equations together with $C_{0,0}=1$ yield all of the coefficients.

Proof of Proposition 18. Case i): For case i) with $K_{q}=0$ for all $q$, the value function is of the form:

$$
h_{q}^{\psi}(t)=\frac{1}{\kappa} \log \left(\sum_{n=0}^{q} C_{q, n}(T-t)^{n}\right)
$$

where each $C_{q, n}>0$, and the feedback form of the optimal spread is:

$$
\delta^{*}(t, q ; \psi)=\frac{1}{\varphi_{\kappa}} \log \left(1+\frac{\varphi_{\kappa}}{\kappa}\right)-h_{q-1}^{\psi}(t)+h_{q}^{\psi}(t)
$$

Substituting the value function into the feedback expression gives:

$$
\delta^{*}(t, q ; \psi)=\frac{1}{\varphi_{\kappa}} \log \left(1+\frac{\varphi_{\kappa}}{\kappa}\right)+\frac{1}{\kappa} \log \left(\frac{\sum_{n=0}^{q} C_{q, n}(T-t)^{n}}{\sum_{n=0}^{q-1} C_{q-1, n}(T-t)^{n}}\right)
$$

As $T-t \rightarrow \infty$, the argument of the logarithm approaches $\infty$ since the numerator is a polynomial of higher degree than the denominator. The argument of the logarithm clearly grows as $\frac{C_{q, q}}{C_{q-1, q-1}}(T-t)$. With the expression for $C_{q, n}$ from Proposition 17, this means the spread grows as:

$$
\delta^{*}(t, q ; \psi) \approx \frac{1}{\kappa} \log \left(\frac{\xi}{q}(T-t)\right)
$$

Case ii): For case ii) with $K_{q}<0$ for all $q>0$, the value function is of the form:

$$
h_{q}^{\psi}(t)=\frac{1}{\kappa} \log \left(\sum_{n=0}^{q} C_{q, n} e^{K_{n}(T-t)}\right)
$$

and so the optimal spread is equal to:

$$
\delta^{*}(t, q ; \psi)=\frac{1}{\varphi_{\kappa}} \log \left(1+\frac{\varphi_{\kappa}}{\kappa}\right)+\frac{1}{\kappa} \log \left(\frac{\sum_{n=0}^{q} C_{q, n} e^{K_{n}(T-t)}}{\sum_{n=0}^{q-1} C_{q-1, n} e^{K_{n}(T-t)}}\right)
$$


Since each $K_{n}$ is negative (except for $K_{0}$ which is equal to 0 ), this clearly converges as $(T-t) \rightarrow \infty$ to:

$$
\begin{aligned}
\delta^{*}(t, q ; \psi) & \rightarrow \frac{1}{\varphi_{\kappa}} \log \left(1+\frac{\varphi_{\kappa}}{\kappa}\right)+\frac{1}{\kappa} \log \left(\frac{C_{q, 0}}{C_{q-1,0}}\right) \\
& =\frac{1}{\varphi_{\kappa}} \log \left(1+\frac{\varphi_{\kappa}}{\kappa}\right)+\frac{1}{\kappa} \log \left(\frac{-\xi}{K_{q}}\right)
\end{aligned}
$$

\section{B.3 Proofs from Chapter 4}

Proof of Proposition 21. The likelihood function $\mathcal{L}(Z)$ is the same as in the proof of Proposition 20. When maximizing the log likelihood, additional Lagrange multipliers are introduced. $L_{i}^{G}$ handles each of the constraints in equation 4.4, and $L_{i, j}^{p}$ handles equation 4.5. The multipliers $L_{i}$ are still present to ensure that transition probabilities add to 1 :

$$
\begin{aligned}
\log \mathcal{L}(Z)= & \sum_{k=0}^{M-1} \log \left(G_{Z_{\tau_{k}}, Z_{\tau_{k}}}\right)-G_{Z_{\tau_{k}}, Z_{\tau_{k}}}\left(\tau_{k+1}-\tau_{k}\right)+\log \left(p_{Z_{\tau_{k}}, Z_{\tau_{k+1}}}\right) \\
& +\sum_{i=1}^{n} L_{i}\left(\sum_{j \neq i} p_{i, j}-1\right)+\sum_{i=1}^{n} L_{i}^{G}\left(G_{i, i}-G_{n-i+1, n-i+1}\right) \\
& +\sum_{i, j} L_{i, j}^{P}\left(p_{i, j}-p_{n-i+1, n-j+1}\right)
\end{aligned}
$$

Solving for $G_{i, i}$ : First order conditions in $G_{i, i}$ give:

$$
\begin{aligned}
0 & =\sum_{k=0}^{M-1}\left(-\mathbb{1}_{Z_{\tau_{k}}=i}\left(\tau_{k+1}-\tau_{k}\right)+\frac{\mathbb{1}_{Z_{\tau_{k}}=i}}{G_{i, i}}\right)+L_{i}^{G}-L_{n-i+1}^{G} \\
0 & =-T_{i}+\frac{N_{i}}{G_{i, i}}+L_{i}^{G}-L_{n-i+1}^{G} \\
G_{i, i} & =\frac{N_{i}}{T_{i}-L_{i}^{G}+L_{n-i+1}^{G}}
\end{aligned}
$$

Similarly, first order conditions in $G_{n-i+1, n-i+1}$ give:

$$
G_{n-i+1, n-i+1}=\frac{N_{n-i+1}}{T_{n-i+1}-L_{n-i+1}^{G}+L_{i}^{G}}
$$


The symmetry constraint forces these two expressions to be equal, and setting them so allows for $L_{i}^{G}-L_{n-i+1}^{G}$ to be solved for:

$$
\begin{aligned}
\frac{N_{i}}{T_{i}-L_{i}^{G}+L_{n-i+1}^{G}} & =\frac{N_{n-i+1}}{T_{n-i+1}-L_{n-i+1}^{G}+L_{i}^{G}} \\
\frac{T_{i}-L_{i}^{G}+L_{n-i+1}^{G}}{N_{i}} & =\frac{T_{n-i+1}-L_{n-i+1}^{G}+L_{i}^{G}}{N_{n-i+1}} \\
N_{n-i+1}\left(T_{i}-L_{i}^{G}+L_{n-i+1}^{G}\right) & =N_{i}\left(T_{n-i+1}-L_{n-i+1}^{G}+L_{i}^{G}\right) \\
\left(N_{n-i+1}+N_{i}\right)\left(L_{i}^{G}-L_{n-i+1}^{G}\right) & =-N_{i} T_{n-i+1}+N_{n-i+1} T_{i} \\
L_{i}^{G}-L_{n-i+1}^{G} & =\frac{-N_{i} T_{n-i+1}+N_{n-i+1} T_{i}}{\left(N_{n-i+1}+N_{i}\right)}
\end{aligned}
$$

Substituting this into the expression for $G_{i, i}$ gives:

$$
\begin{aligned}
G_{i, i} & =\frac{N_{i}}{T_{i}-L_{i}^{G}+L_{n-i+1}^{G}} \\
G_{i, i} & =\frac{N_{i}}{T_{i}-\frac{-N_{i} T_{n-i+1}+N_{n-i+1} T_{i}}{\left(N_{n-i+1}+N_{i}\right)}} \\
G_{i, i} & =\frac{N_{i}\left(N_{n-i+1}+N_{i}\right)}{T_{i}\left(N_{n-i+1}+N_{i}\right)+N_{i} T_{n-i+1}-N_{n-i+1} T_{i}} \\
G_{i, i} & =\frac{N_{i}+N_{n-i+1}}{T_{i}+T_{n-i+1}}
\end{aligned}
$$

This is the first desired expression.

Solving for $p_{i, j}$ : First order conditions in $p_{i, j}$ give:

$$
\begin{aligned}
0 & =\sum_{k=0}^{M-1} \frac{1}{p_{i, j}} \mathbb{1}_{Z_{\tau_{k}}=i, Z_{\tau_{k+1}}=j}+L_{i}+L_{i, j}^{P}-L_{n-i+1, n-j+1}^{P} \\
0 & =\frac{N_{i, j}}{p_{i, j}}+L_{i}+L_{i, j}^{P}-L_{n-i+1, n-j+1}^{P} \\
p_{i, j} & =\frac{-N_{i, j}}{L_{i}+L_{i, j}^{P}-L_{n-i+1, n-j+1}^{P}}
\end{aligned}
$$

Similarly, first order conditions in $p_{n-i+1, n-j+1}$ give:

$$
p_{n-i+1, n-j+1}=\frac{-N_{n-i+1, n-j+1}}{L_{n-i+1}-L_{i, j}^{P}+L_{n-i+1, n-j+1}^{P}}
$$


The symmetry constraint forces these two expressions to be equal. Setting them so allows for $L_{i, j}^{P}-L_{n-i+1, n-j+1}^{P}$ to be solved for:

$$
\begin{aligned}
\frac{-N_{i, j}}{L_{i}+L_{i, j}^{P}-L_{n-i+1, n-j+1}^{P}} & =\frac{-N_{n-i+1, n-j+1}}{L_{n-i+1}-L_{i, j}^{P}+L_{n-i+1, n-j+1}^{P}} \\
\frac{L_{i}+L_{i, j}^{P}-L_{n-i+1, n-j+1}^{P}}{N_{i, j}} & =\frac{L_{n-i+1}-L_{i, j}^{P}+L_{n-i+1, n-j+1}^{P}}{N_{n-i+1, n-j+1}} \\
N_{n-i+1, n-j+1}\left(L_{i}+L_{i, j}^{P}-L_{n-i+1, n-j+1}^{P}\right) & =N_{i, j}\left(L_{n-i+1}-L_{i, j}^{P}+L_{n-i+1, n-j+1}^{P}\right) \\
\left(N_{i, j}+N_{n-i+1, n-j+1}\right)\left(L_{i, j}^{P}-L_{n-i+1, n-j+1}^{P}\right) & =N_{i, j} L_{n-i+1}-N_{n-i+1, n-j+1} L_{i} \\
L_{i, j}^{P}-L_{n-i+1, n-j+1}^{P} & =\frac{N_{i, j} L_{n-i+1}-N_{n-i+1, n-j+1} L_{i}}{N_{i, j}+N_{n-i+1, n-j+1}}
\end{aligned}
$$

Substituting this back into equation (B.28) gives:

$$
\begin{aligned}
p_{i, j} & =\frac{-N_{i, j}}{L_{i}+L_{i, j}^{P}-L_{n-i+1, n-j+1}^{P}} \\
p_{i, j} & =\frac{-N_{i, j}}{L_{i}+\frac{N_{i, j} L_{n-i+1}-N_{n-i+1, n-j+1} L_{i}}{N_{i, j}+N_{n-i+1, n-j+1}}} \\
p_{i, j} & =\frac{-N_{i, j}\left(N_{i, j}+N_{n-i+1, n-j+1}\right)}{L_{i}\left(N_{i, j}+N_{n-i+1, n-j+1}\right)+N_{i, j} L_{n-i+1}-N_{n-i+1, n-j+1} L_{i}} \\
p_{i, j} & =\frac{-\left(N_{i, j}+N_{n-i+1, n-j+1}\right)}{L_{i}+L_{n-i+1}}
\end{aligned}
$$

And now substituting this into the summation constraint gives:

$$
\begin{aligned}
\sum_{j \neq i} p_{i, j} & =1 \\
\sum_{j \neq i} \frac{-\left(N_{i, j}+N_{n-i+1, n-j+1}\right)}{L_{i}+L_{n-i+1}} & =1 \\
L_{i}+L_{n-i+1} & =\sum_{j \neq i}-\left(N_{i, j}+N_{n-i+1, n-j+1}\right) \\
L_{i}+L_{n-i+1} & =-\left(N_{i}+N_{n-i+1}\right)
\end{aligned}
$$

This is substituted into equation (B.29) to give:

$$
p_{i, j}=\frac{N_{i, j}+N_{n-i+1, n-j+1}}{N_{i}+N_{n-i+1}}
$$


which is the second desired expression.

Solving for $\lambda^{ \pm}(i)$ : Solving for the market order intensities follows in a similar fashion. The likelihood function for a sequence of market orders at times $\tau_{k}^{ \pm}$is the same as in the proof of Proposition 20, but Lagrange multipliers $L_{i}^{ \pm}$are introduced to enforce the constraint $\lambda^{ \pm}(i)=\lambda^{\mp}(n-i+1)$ :

$$
\log \mathcal{L}\left(\tau^{ \pm}\right)=\sum_{ \pm} \sum_{k=1}^{N^{ \pm}}\left(\log \left(\lambda_{\tau_{k}^{ \pm}}^{ \pm}\right)-\int_{\tau_{k-1}^{ \pm}}^{\tau_{k}^{ \pm}} \lambda_{t}^{ \pm} d t\right)+\sum_{ \pm} \sum_{i=1}^{n} L_{i}^{ \pm}\left(\lambda^{ \pm}(i)-\lambda^{\mp}(n-i+1)\right)
$$

First order conditions in $\lambda^{ \pm}(i)$ give:

$$
\begin{aligned}
0 & =\sum_{k=1}^{N^{ \pm}}\left(\frac{1}{\lambda^{ \pm}(i)} \mathbb{1}_{Z_{\tau_{k}}=i}\right)-T_{i}^{ \pm}+L_{i}^{ \pm}-L_{n-i+1}^{\mp} \\
0 & =\frac{N_{i}^{ \pm}}{\lambda^{ \pm}(i)}-T_{i}^{ \pm}+L_{i}^{ \pm}-L_{n-i+1}^{\mp} \\
\lambda^{ \pm}(i) & =\frac{N_{i}^{ \pm}}{T_{i}^{ \pm}-L_{i}^{ \pm}+L_{n-i+1}^{\mp}}
\end{aligned}
$$

Similarly, the expression for $\lambda^{\mp}(n-i+1)$ is:

$$
\lambda^{\mp}(n-i+1)=\frac{N_{n-i+1}^{\mp}}{T_{n-i+1}^{\mp}+L_{i}^{ \pm}-L_{n-i+1}^{\mp}}
$$

Enforcing the constraint $\lambda^{ \pm}(i)=\lambda^{\mp}(n-i+1)$ and solving for $L_{i}^{ \pm}-L_{n-i+1}^{\mp}$ gives:

$$
\begin{aligned}
\frac{N_{i}^{ \pm}}{T_{i}^{ \pm}-L_{i}^{ \pm}+L_{n-i+1}^{\mp}} & =\frac{N_{n-i+1}^{\mp}}{T_{n-i+1}^{\mp}+L_{i}^{ \pm}-L_{n-i+1}^{\mp}} \\
\frac{T_{i}^{ \pm}-L_{i}^{ \pm}+L_{n-i+1}^{\mp}}{N_{i}^{ \pm}} & =\frac{T_{n-i+1}^{\mp}+L_{i}^{ \pm}-L_{n-i+1}^{\mp}}{N_{n-i+1}^{\mp}} \\
N_{n-i+1}^{\mp}\left(T_{i}^{ \pm}-L_{i}^{ \pm}+L_{n-i+1}^{\mp}\right) & =N_{i}^{ \pm}\left(T_{n-i+1}^{\mp}+L_{i}^{ \pm}-L_{n-i+1}^{\mp}\right) \\
\left(N_{i}^{ \pm}+N_{n-i+1}^{\mp}\right)\left(L_{i}^{ \pm}-L_{n-i+1}^{\mp}\right) & =N_{n-i+1}^{\mp} T_{i}^{ \pm}-N_{i}^{ \pm} T_{n-i+1}^{\mp} \\
L_{i}^{ \pm}-L_{n-i+1}^{\mp} & =\frac{N_{n-i+1}^{\mp} T_{i}^{ \pm}-N_{i}^{ \pm} T_{n-i+1}^{\mp}}{N_{i}^{ \pm}+N_{n-i+1}^{\mp}}
\end{aligned}
$$


Substituting this into equation (B.30) gives:

$$
\begin{aligned}
\lambda^{ \pm}(i) & =\frac{N_{i}^{ \pm}\left(N_{i}^{ \pm}+N_{n-i+1}^{\mp}\right)}{T_{i}^{ \pm}\left(N_{i}^{ \pm}+N_{n-i+1}^{\mp}\right)-\left(N_{n-i+1}^{\mp} T_{i}^{ \pm}-N_{i}^{ \pm} T_{n-i+1}^{\mp}\right)} \\
\lambda^{ \pm}(i) & =\frac{N_{i}^{ \pm}+N_{n-i+1}^{\mp}}{T_{i}^{ \pm}+T_{n-i+1}^{\mp}}
\end{aligned}
$$

which is the third and final desired expression.

\section{B.4 Proofs from Chapter 5}

Proof of Proposition 22. The term which requires optimization is:

$$
\sup _{\gamma^{ \pm} \in\{0,1\}} \inf _{g^{ \pm}}\left\{e^{g^{ \pm}} \lambda^{ \pm}(Z)\left(\gamma^{ \pm} \frac{\Delta}{2}-\left(\gamma^{ \pm} \mp q\right) \epsilon_{Z}^{ \pm}+h_{q \mp \gamma^{ \pm}, Z}^{\varphi_{\lambda}}-h_{q, Z}^{\varphi_{\lambda}}\right)+\frac{\lambda^{ \pm}(Z)}{\varphi_{\lambda}}\left(e^{g^{ \pm}}\left(g^{ \pm}-1\right)+1\right)\right\}
$$

Solving for $g^{ \pm}$: First order conditions in $g^{ \pm}$give:

$$
\begin{aligned}
0 & =e^{g^{ \pm}} \lambda^{ \pm}(Z)\left(\gamma^{ \pm} \frac{\Delta}{2}-\left(\gamma^{ \pm} \mp q\right) \epsilon_{Z}^{ \pm}+h_{q \mp \gamma^{ \pm}, Z}^{\varphi_{\lambda}}-h_{q, Z}^{\varphi_{\lambda}}\right)+\frac{\lambda^{ \pm}(Z)}{\varphi_{\lambda}} e^{g^{ \pm}} g^{ \pm} \\
g^{ \pm} & =-\varphi_{\lambda}\left(\gamma^{ \pm} \frac{\Delta}{2}-\left(\gamma^{ \pm} \mp q\right) \epsilon_{Z}^{ \pm}+h_{q \mp \gamma^{ \pm}, Z}^{\varphi_{\lambda}}-h_{q, Z}^{\varphi_{\lambda}}\right)
\end{aligned}
$$


To check that this expression is indeed a minimizer, take a second derivative with respect to $g^{ \pm}$, and evaluate at this value to get:

$$
\begin{aligned}
& e^{g^{ \pm}} \lambda^{ \pm}(Z)\left(\gamma^{ \pm} \frac{\Delta}{2}-\left(\gamma^{ \pm} \mp q\right) \epsilon_{Z}^{ \pm}+h_{q \mp \gamma^{ \pm}, Z}^{\varphi_{\lambda}}-h_{q, Z}^{\varphi_{\lambda}}\right)+\frac{\lambda^{ \pm}(Z)}{\varphi_{\lambda}} e^{g^{ \pm}} g^{ \pm}+\frac{\lambda^{ \pm}(Z)}{\varphi_{\lambda}} e^{g^{ \pm}} \\
= & e^{-\varphi_{\lambda}\left(\gamma^{ \pm} \frac{\Delta}{2}-\left(\gamma^{ \pm} \mp q\right) \epsilon_{Z}^{ \pm}+h_{q \mp \gamma^{ \pm}, Z}^{\varphi_{\lambda}}-h_{q, Z}^{\varphi_{\lambda}}\right)} \lambda^{ \pm}(Z)\left(\gamma^{ \pm} \frac{\Delta}{2}-\left(\gamma^{ \pm} \mp q\right) \epsilon_{Z}^{ \pm}+h_{q \mp \gamma^{ \pm}, Z}^{\varphi_{\lambda}}-h_{q, Z}^{\varphi_{\lambda}}\right) \\
& -\lambda^{ \pm}(Z) e^{-\varphi_{\lambda}\left(\gamma^{ \pm} \frac{\Delta}{2}-\left(\gamma^{ \pm} \mp q\right) \epsilon_{Z}^{ \pm}+h_{q \mp \gamma^{ \pm}, Z^{\varphi}}^{\varphi_{\lambda}}-h_{q, Z}^{\varphi_{\lambda}}\right)}\left(\gamma^{ \pm} \frac{\Delta}{2}-\left(\gamma^{ \pm} \mp q\right) \epsilon_{Z}^{ \pm}+h_{q \mp \gamma^{ \pm}, Z}^{\varphi_{\lambda}}-h_{q, Z}^{\varphi_{\lambda}}\right) \\
& +\frac{\lambda^{ \pm}(Z)}{\varphi_{\lambda}} e^{-\varphi_{\lambda}\left(\gamma^{ \pm} \frac{\Delta}{2}-\left(\gamma^{ \pm} \mp q\right) \epsilon_{Z}^{ \pm}+h_{q \mp \gamma^{ \pm}, Z}^{\varphi_{\lambda}}-h_{q, Z}^{\varphi_{\lambda}}\right)} \\
= & \frac{\lambda^{ \pm}(Z)}{\varphi_{\lambda}} e^{-\varphi_{\lambda}\left(\gamma^{ \pm} \frac{\Delta}{2}-\left(\gamma^{ \pm} \mp q\right) \epsilon_{Z}^{ \pm}+h_{q \mp \gamma^{ \pm}, Z^{\prime}}^{\varphi_{\lambda}}-h_{q, Z}^{\varphi_{\lambda}}\right)} \\
> & 0
\end{aligned}
$$

And so this value of $g^{ \pm}$minimizes the expression of interest.

Solving for $\gamma^{ \pm}$: Substituting this value of $g^{ \pm}$back into the original term gives:

$$
\sup _{\gamma^{ \pm} \in\{0,1\}}\left\{\frac{\lambda^{ \pm}(Z)}{\varphi_{\lambda}}\left(1-e^{-\varphi_{\lambda}\left(\gamma^{ \pm} \frac{\Delta}{2}-\left(\gamma^{ \pm} \mp q\right) \epsilon_{Z}^{ \pm}+h_{q \mp \gamma^{ \pm}, Z^{\varphi_{\lambda}}}-h_{q, Z}^{\varphi_{\lambda}}\right)}\right)\right\}
$$

And now some simplifications are performed:

$$
\begin{aligned}
& \underset{\gamma^{ \pm} \in\{0,1\}}{\arg \max }\left\{\frac{\lambda^{ \pm}(Z)}{\varphi_{\lambda}}\left(1-\exp \left\{-\varphi_{\lambda}\left(\gamma^{ \pm} \frac{\Delta}{2}-\left(\gamma^{ \pm} \mp q\right) \epsilon_{Z}^{ \pm}+h_{q \mp \gamma^{ \pm}, Z}^{\varphi_{\lambda}}-h_{q, Z}^{\varphi_{\lambda}}\right)\right\}\right)\right\} \\
= & \underset{\gamma^{ \pm} \in\{0,1\}}{\arg \min }\left\{\exp \left\{-\varphi_{\lambda}\left(\gamma^{ \pm} \frac{\Delta}{2}-\left(\gamma^{ \pm} \mp q\right) \epsilon_{Z}^{ \pm}+h_{q \mp \gamma^{ \pm}, Z}^{\varphi_{\lambda}}-h_{q, Z}^{\varphi_{\lambda}}\right)\right\}\right\} \\
= & \underset{\gamma^{ \pm} \in\{0,1\}}{\arg \max }\left\{\gamma^{ \pm} \frac{\Delta}{2}-\left(\gamma^{ \pm} \mp q\right) \epsilon_{Z}^{ \pm}+h_{q \mp \gamma^{ \pm}, Z}^{\varphi_{\lambda}}-h_{q, Z}^{\varphi_{\lambda}}\right\} \\
= & \underset{\gamma^{ \pm} \in\{0,1\}}{\arg \max }\left\{\gamma^{ \pm} \frac{\Delta}{2}-\gamma^{ \pm} \epsilon_{Z}^{ \pm}+h_{q \mp \gamma^{ \pm}, Z}^{\varphi_{\lambda}}-h_{q, Z}^{\varphi_{\lambda}}\right\} \\
= & \left\{\begin{array}{cc}
1, & \frac{\Delta}{2}-\epsilon_{Z}^{ \pm}+h_{q \mp 1, Z}^{\varphi_{\lambda}}(t)-h_{q, Z}^{\varphi_{\lambda}}(t)>0 \\
0, & \frac{\Delta}{2}-\epsilon_{Z}^{ \pm}+h_{q \mp 1, Z}^{\varphi_{\lambda}}(t)-h_{q, Z}^{\varphi_{\lambda}}(t) \leq 0
\end{array}\right.
\end{aligned}
$$

which is the desired result. 
Proof of Proposition 23. The quantity to be optimized is:

$$
\begin{aligned}
\sup _{\gamma^{ \pm} \in\{0,1\}} \inf _{g^{ \pm} \in \mathcal{G}_{Z}^{ \pm}}\left\{\lambda ^ { \pm } ( Z ) \left(\gamma^{ \pm} \frac{\Delta}{2}-\left(\gamma^{ \pm} \mp q\right)\right.\right. & \left.\int_{-\infty}^{\infty} y e^{g^{ \pm}(y)} F_{Z}^{ \pm}(y) d y+h_{q \mp \gamma^{ \pm}, Z}^{\varphi_{\epsilon}}-h_{q, Z}^{\varphi_{\epsilon}}\right) \\
& \left.+\frac{\lambda^{ \pm}(Z)}{\varphi_{\epsilon}} \int_{-\infty}^{\infty} e^{g^{ \pm}(y)}\left(g^{ \pm}(y)-1\right)+1 F_{Z}^{ \pm}(y) d y\right\}
\end{aligned}
$$

Recall that the sets $\mathcal{G}_{Z}^{ \pm}$are defined by:

$$
\mathcal{G}_{Z}^{ \pm}=\left\{g: \int_{-\infty}^{\infty} e^{g(y)} F_{Z}^{ \pm}(y) d y=1 \text { and } \int_{-\infty}^{\infty} y e^{g(y)} F_{Z}^{ \pm}(y) d y<\infty\right\} .
$$

Solving for $g^{ \pm}$: The equality constraint is handled by introducing Lagrange multipliers $L^{ \pm}$and optimizing over an unconstrained set:

$$
\begin{aligned}
& \inf _{g^{ \pm}}\left\{\lambda^{ \pm}(Z)\left(\gamma^{ \pm} \frac{\Delta}{2}-\left(\gamma^{ \pm} \mp q\right) \int_{-\infty}^{\infty} y e^{g^{ \pm}(y)} F_{Z}^{ \pm}(y) d y+h_{q \mp \gamma^{ \pm}, Z}^{\varphi_{\epsilon}}-h_{q, Z}^{\varphi_{\epsilon}}\right)\right. \\
& \left.\quad+\frac{\lambda^{ \pm}(Z)}{\varphi_{\epsilon}} \int_{-\infty}^{\infty} e^{g^{ \pm}(y)}\left(g^{ \pm}(y)-1\right)+1 F_{Z}^{ \pm}(y) d y+L^{ \pm}\left(\int_{-\infty}^{\infty} e^{g^{ \pm}(y)} F_{Z}^{ \pm}(y) d y-1\right)\right\}
\end{aligned}
$$

Since $g^{ \pm}$only appears within integrals, the integrand is simply minimized with respect to $g^{ \pm}(y)$ for each value of $y$ :

$$
\inf _{g^{ \pm}(y)}\left\{\left( \pm q-\gamma^{ \pm}\right) y e^{g^{ \pm}(y)}+\frac{1}{\varphi_{\epsilon}}\left(e^{g^{ \pm}(y)}\left(g^{ \pm}(y)-1\right)+1\right)+L^{ \pm}\left(e^{g^{ \pm}(y)}-1\right)\right\}
$$

First order conditions give:

$$
\begin{aligned}
0 & =\left( \pm q-\gamma^{ \pm}\right) y e^{g^{ \pm}(y)}+\frac{1}{\varphi_{\epsilon}} e^{g^{ \pm}(y)} g^{ \pm}(y)+L^{ \pm} e^{g^{ \pm}(y)} \\
g^{ \pm}(y) & =-L^{ \pm} \varphi_{\epsilon}+\left(\gamma^{ \pm} \mp q\right) \varphi_{\epsilon} y
\end{aligned}
$$


Solving for $L^{ \pm}$: Substituting this into the integral constraint to solve for $L^{ \pm}$gives:

$$
\begin{aligned}
1 & =\int_{-\infty}^{\infty} e^{g^{ \pm}(y)} F_{Z}^{ \pm}(y) d y \\
1 & =\int_{-\infty}^{\infty} e^{-L^{ \pm} \varphi_{\epsilon}+\left(\gamma^{ \pm} \mp q\right) \varphi_{\epsilon} y} F_{Z}^{ \pm}(y) d y \\
e^{L^{ \pm} \varphi_{\epsilon}} & =\int_{-\infty}^{\infty} e^{\left(\gamma^{ \pm} \mp q\right) \varphi_{\epsilon} y} F_{Z}^{ \pm}(y) d y \\
L^{ \pm} & =\frac{1}{\varphi_{\epsilon}} \log \left(\int_{-\infty}^{\infty} e^{\left(\gamma^{ \pm} \mp q\right) \varphi_{\epsilon} y} F_{Z}^{ \pm}(y) d y\right)
\end{aligned}
$$

By assumption in the proposition, $\int_{-\infty}^{\infty} y^{k} e^{\left(\gamma^{ \pm} \mp q\right) \varphi_{\epsilon} y} F_{Z}^{ \pm}(y) d y<\infty$ for $k=0,1$. Substituting the value of the Lagrange multiplier into equation (B.32) gives:

$$
g^{ \pm}(y)=-\log \left(\int_{-\infty}^{\infty} e^{\left(\gamma^{ \pm} \mp q\right) \varphi_{\epsilon} y} F_{Z}^{ \pm}(y) d y\right)+\left(\gamma^{ \pm} \mp q\right) \varphi_{\epsilon} y
$$

Substituting this expression back into equation (B.31) gives:

$$
\sup _{\gamma^{ \pm} \in\{0,1\}}\left\{\lambda^{ \pm}(Z)\left(\gamma^{ \pm} \frac{\Delta}{2}-\frac{1}{\varphi_{\epsilon}} \log \left(\int_{-\infty}^{\infty} e^{\left(\gamma^{ \pm} \mp q\right) \varphi_{\epsilon} y} F_{Z}^{ \pm}(y) d y\right)+h_{q \mp \gamma^{ \pm}, Z}^{\varphi_{\epsilon}}-h_{q, Z}^{\varphi_{\epsilon}}\right)\right\}
$$

The choice of whether to let $\gamma^{ \pm}$be 0 or 1 depends on the comparison of two values. $\gamma^{ \pm}$ is chosen to be 1 if:

$$
\begin{aligned}
\frac{\Delta}{2}-\frac{1}{\varphi_{\epsilon}} \log \left(\int_{-\infty}^{\infty} e^{(1 \mp q) \varphi_{\epsilon} y} F_{Z}^{ \pm}(y) d y\right)+h_{q \mp 1, Z}^{\varphi_{\epsilon}}-h_{q, Z}^{\varphi_{\epsilon}} & >-\frac{1}{\varphi_{\epsilon}} \log \left(\int_{-\infty}^{\infty} e^{\mp q \varphi_{\epsilon} y} F_{Z}^{ \pm}(y) d y\right) \\
\varphi_{\epsilon}\left(\frac{\Delta}{2}+h_{q \mp 1, Z}^{\varphi_{\epsilon}}-h_{q, Z}^{\varphi_{\epsilon}}\right) & >\log \left(\frac{\int_{-\infty}^{\infty} e^{(1 \mp q) \varphi_{\epsilon} y} F_{Z}^{ \pm}(y) d y}{\int_{-\infty}^{\infty} e^{\mp q \varphi_{\epsilon} y} F_{Z}^{ \pm}(y) d y}\right) \\
\exp \left\{\varphi_{\epsilon}\left(\frac{\Delta}{2}+h_{q \mp 1, Z}^{\varphi_{\epsilon}}-h_{q, Z}^{\varphi_{\epsilon}}\right)\right\} & >\frac{\int_{-\infty}^{\infty} e^{(1 \mp q) \varphi_{\epsilon} y} F_{Z}^{ \pm}(y) d y}{\int_{-\infty}^{\infty} e^{\mp q \varphi_{\epsilon} y} F_{Z}^{ \pm}(y) d y}
\end{aligned}
$$

and $\gamma^{ \pm}$is chosen to be zero otherwise, as desired.

Proof of Proposition 24. The term of interest to be optimized is: The quantity to be 
optimized is:

$$
\begin{aligned}
\sup _{\gamma^{ \pm} \in\{0,1\}} \inf _{g^{ \pm} \in \mathcal{G}_{Z}^{ \pm}}\left\{\lambda ^ { \pm } ( Z ) \left(\gamma^{ \pm} \frac{\Delta}{2}-\left(\gamma^{ \pm} \mp q\right)\right.\right. & \left.\int_{-\infty}^{\infty} y e^{g^{ \pm}(y)} F_{Z}^{ \pm}(y) d y+h_{q \mp \gamma^{ \pm}, Z}^{\varphi_{\epsilon}}-h_{q, Z}^{\varphi_{\epsilon}}\right) \\
& \left.+\frac{\lambda^{ \pm}(Z)}{\varphi_{\epsilon}} \int_{-\infty}^{\infty} e^{g^{ \pm}(y)}\left(g^{ \pm}(y)-1\right)+1 F_{Z}^{ \pm}(y) d y\right\}
\end{aligned}
$$

Recall that the sets $\mathcal{G}_{Z}^{ \pm}$are defined by:

$$
\mathcal{G}_{Z}^{ \pm}=\left\{g: \int_{-\infty}^{\infty} e^{g(y)} F_{Z}^{ \pm}(y) d y=1 \text { and } \int_{-\infty}^{\infty} y e^{g(y)} F_{Z}^{ \pm}(y) d y<\infty\right\} .
$$

If the proof were to proceed as in the proof of Proposition 23, it is possible that issues would arise with certain integrals having infinite values. This was avoided in the previous proposition by assuming that $\varphi_{\epsilon}$ was sufficiently small that all associated integrals were finite. Drawing from the conclusion of that proposition, define $g_{\phi}^{ \pm}$as:

$$
g_{\phi}^{ \pm}\left(y ; q, \gamma^{ \pm}\right)=-\log \left(\int_{-\infty}^{\infty} e^{\left(\gamma^{ \pm} \mp q\right) \phi y} F_{Z}^{ \pm}(y) d y\right)+\left(\gamma^{ \pm} \mp q\right) \phi y .
$$

By the condition on $F_{Z}^{ \pm}$from equation (5.6), $g_{\phi}^{ \pm}\left(y ; q, \gamma^{ \pm}\right)$is well defined for sufficiently small $\phi$ (possibly depending on $q$ and $\gamma^{ \pm}$), and $g_{\phi}^{ \pm}\left(y ; q, \gamma^{ \pm}\right) \in \mathcal{G}_{Z}^{ \pm}$when it is defined. Let $\left[0, \phi_{0}\right)$ be the interval for which $g_{\phi}^{ \pm}\left(y ; q, \gamma^{ \pm}\right)$is well defined and for which:

$$
\int_{-\infty}^{\infty}|y| e^{\left(\gamma^{ \pm} \mp q\right) \phi y} F_{Z}^{ \pm}(y) d y<\infty
$$

This interval may possibly depend on $q$ and $\gamma^{ \pm}$. If $\phi_{0}>\varphi_{\epsilon}$, then taking $\phi \rightarrow \varphi_{\epsilon}$ causes no convergence issues and $g^{ \pm *}=g_{\phi}^{ \pm}$as in the proof of Proposition 23.

Suppose $\phi_{0} \leq \varphi_{\epsilon}$ for particular values of $q$ and $\gamma^{ \pm}$. There are two possibilities of interest as $\phi \rightarrow \phi_{0}$ :

$$
\int_{-\infty}^{\infty}|y| e^{\left(\gamma^{ \pm} \mp q\right) \phi y} F_{Z}^{ \pm}(y) d y \rightarrow \infty \quad \text { and } \quad \int_{-\infty}^{\infty} e^{\left(\gamma^{ \pm} \mp q\right) \phi y} F_{Z}^{ \pm}(y) d y \rightarrow \int_{-\infty}^{\infty} e^{\left(\gamma^{ \pm} \mp q\right) \phi_{0} y} F_{Z}^{ \pm}(y) d y<\infty,
$$

or:

$$
\int_{-\infty}^{\infty}|y| e^{\left(\gamma^{ \pm} \mp q\right) \phi y} F_{Z}^{ \pm}(y) d y \rightarrow \infty \quad \text { and } \quad \int_{-\infty}^{\infty} e^{\left(\gamma^{ \pm} \mp q\right) \phi y} F_{Z}^{ \pm}(y) d y \rightarrow \infty
$$

To see how each of these possibilities affect the term to be optimized, let $T_{\phi}=\int_{-\infty}^{\infty} e^{\left(\gamma^{ \pm} \mp q\right) \phi y} F_{Z}^{ \pm}(y) d y$ 
and substitute $g_{\phi}^{ \pm}$into equation (B.33):

$$
\begin{aligned}
& \lambda^{ \pm}(Z)\left(\gamma^{ \pm} \frac{\Delta}{2}-\left(\gamma^{ \pm} \mp q\right) \int_{-\infty}^{\infty} y e^{g_{\phi}^{ \pm}(y)} F_{Z}^{ \pm}(y) d y+h_{q \mp \gamma^{ \pm}, Z}^{\varphi_{\epsilon}}-h_{q, Z}^{\varphi_{\epsilon}}\right) \\
& +\frac{\lambda^{ \pm}(Z)}{\varphi_{\epsilon}} \int_{-\infty}^{\infty} e^{g_{\phi}^{ \pm}(y)}\left(g_{\phi}^{ \pm}(y)-1\right)+1 F_{Z}^{ \pm}(y) d y \\
= & \lambda^{ \pm}(Z)\left(\gamma^{ \pm} \frac{\Delta}{2}+h_{q \mp \gamma^{ \pm}, Z}^{\varphi_{\epsilon}}-h_{q, Z}^{\varphi_{\epsilon}}\right)-\lambda^{ \pm}(Z) \frac{\gamma^{ \pm} \mp q}{T_{\phi}} \int_{-\infty}^{\infty} y e^{\left(\gamma^{ \pm} \mp q\right) \phi y} F_{Z}^{ \pm}(y) d y \\
& +\lambda^{ \pm}(Z) \frac{\phi}{\varphi_{\epsilon}} \frac{\gamma^{ \pm} \mp q}{T_{\phi}} \int_{-\infty}^{\infty} y e^{\left(\gamma^{ \pm} \mp q\right) \phi y} F_{Z}^{ \pm}(y) d y-\frac{\lambda^{ \pm}(Z)}{\varphi_{\epsilon}} \log T_{\phi} \\
= & \lambda^{ \pm}(Z)\left(\gamma^{ \pm} \frac{\Delta}{2}+h_{q \mp \gamma^{ \pm}, Z}^{\varphi_{\epsilon}}-h_{q, Z}^{\varphi_{\epsilon}}\right)-\left(1-\frac{\phi}{\varphi_{\epsilon}}\right) \lambda^{ \pm}(Z) \frac{\gamma^{ \pm} \mp q}{T_{\phi}} \int_{-\infty}^{\infty} y e^{\left(\gamma^{ \pm} \mp q\right) \phi y} F_{Z}^{ \pm}(y) d y \\
& -\frac{\lambda^{ \pm}(Z)}{\varphi_{\epsilon}} \log T_{\phi}
\end{aligned}
$$

If $T_{\phi} \rightarrow \infty$ as $\phi \rightarrow \phi_{0}$, then this whole term goes to $-\infty$. On the other hand, if only $\int_{-\infty}^{\infty}|y| e^{\left(\gamma^{ \pm} \mp q\right) \phi y} F_{Z}^{ \pm}(y) d y \rightarrow \infty$ and $\phi_{0}<\varphi_{\epsilon}$ then this whole term goes to $-\infty$. Lastly, if $\phi_{0}=\varphi_{\epsilon}$, then the behaviour of this term depends on:

$$
\left(1-\frac{\phi}{\varphi_{\epsilon}}\right) \frac{\int_{-\infty}^{\infty} y e^{\left(\gamma^{ \pm} \mp q\right) \phi y} F_{Z}^{ \pm}(y) d y}{\int_{-\infty}^{\infty} e^{\left(\gamma^{ \pm} \mp q\right) \phi y} F_{Z}^{ \pm}(y) d y}
$$

as $\phi \rightarrow \varphi_{\epsilon}$. If this quantity goes to $\infty$, then the whole term goes to $-\infty$. It this quantity is bounded, then the whole term goes to a finite value and $g_{\phi}^{ \pm} \rightarrow g^{ \pm *}$.

At this point, there are three possibilities of interest when it comes to choosing $\gamma^{ \pm}$: both choices lead to a finite value, both choices lead to a value of $-\infty$, or $\gamma^{ \pm}=1$ leads a value of $-\infty$ and $\gamma^{ \pm}=0$ leads to a finite value. For the first possibility, everything is finite and proceeds as in Proposition 23. In the second possibility, the market maker is in a state of negative infinite value. This particular combination of $q$ and $Z$ leads to $h_{q, Z}^{\varphi_{\epsilon}}=-\infty$, and due to the mixing caused by regime switching, $h_{q, Z}^{\varphi_{\epsilon}}$ will be equal to $-\infty$ for all $Z$. Finally, the boundary case clearly implies that $\gamma^{ \pm}$should be chosen to be equal to 0 , and this essentially redefines the allowed upper and lower inventory positions as outlined in the statement of the proposition.

Proof of Proposition 25. The feedback expressions for $\gamma^{ \pm}$are equivalent to those of Proposition 19 because that optimization is independent of the one with respect to $g$. 
For the optimization with respect to $g$, the term of interest is:

$$
\inf _{g}\left\{\sum_{k=1}^{n} e^{g(k)} G_{Z, k}\left(h_{q, k}^{\varphi_{G}}-h_{q, Z}^{\varphi_{G}}\right)+\frac{1}{\varphi_{G}} \sum_{k \neq Z}\left(e^{g(k)}(g(k)-1)+1\right) G_{Z, k}\right\}
$$

First order conditions with respect to $g(k)$ give:

$$
\begin{aligned}
0 & =e^{g(k)} G_{Z, k}\left(h_{q, k}^{\varphi_{G}}-h_{q, Z}^{\varphi_{G}}\right)+\frac{1}{\varphi_{G}} e^{g(k)} g(k) G_{Z, k} \\
0 & =\left(h_{q, k}^{\varphi_{G}}-h_{q, Z}^{\varphi_{G}}\right)+\frac{1}{\varphi_{G}} g(k) \\
g(k) & =-\varphi_{G}\left(h_{q, k}^{\varphi_{G}}-h_{q, Z}^{\varphi_{G}}\right)
\end{aligned}
$$

This is easily checked to provide a minimum value by taking a second derivative and substituting this expression into the result.

Proof of Proposition 26. The feedback expressions for $\gamma^{ \pm}$are equivalent to those of Proposition 19 because that optimization is independent of the one with respect to $g$. For the optimization with respect to $g$, the term of interest is:

$$
\inf _{g}\left\{e^{g} \sum_{k=1}^{n} G_{Z, k}\left(h_{q, k}^{\varphi_{G}}-h_{q, Z}^{\varphi_{G}}\right)+\frac{1}{\varphi_{G}} \sum_{k \neq Z}\left(e^{g}(g-1)+1\right) G_{Z, k}\right\}
$$

First order conditions in $g$ give:

$$
\begin{aligned}
& 0=e^{g} \sum_{k=1}^{n} G_{Z, k}\left(h_{q, k}^{\varphi_{R}}-h_{q, Z}^{\varphi_{R}}\right)+\frac{1}{\varphi_{R}} e^{g} g \sum_{k \neq Z} G_{Z, k} \\
& 0=\sum_{k=1}^{n} G_{Z, k}\left(h_{q, k}^{\varphi_{R}}-h_{q, Z}^{\varphi_{R}}\right)+\frac{1}{\varphi_{R}} g \sum_{k \neq Z} G_{Z, k} \\
& g=-\varphi_{R} \frac{\sum_{k=1}^{n} G_{Z, k}\left(h_{q, k}^{\varphi_{R}}-h_{q, Z}^{\varphi_{R}}\right)}{\sum_{k \neq Z} G_{Z, k}}
\end{aligned}
$$

This is easily checked to provide a minimum value by taking a second derivative and substituting this expression into the result.

Proof of Proposition 27. The feedback expressions for $\gamma^{ \pm}$are equivalent to those of Proposition 19 because that optimization is independent of the one with respect to $g$. 
Solving for $g$ : For the optimization with respect to $g$, the term of interest is:

$$
\inf _{g \in \mathcal{G}_{Z}}\left\{\sum_{k=1}^{n} e^{g(k)} G_{Z, k}\left(h_{q, k}^{\varphi_{P}}-h_{q, Z}^{\varphi_{P}}\right)+\frac{1}{\varphi_{P}} \sum_{k \neq Z}\left(e^{g(k)}(g(k)-1)+1\right) G_{Z, k}\right\}
$$

Introducing Lagrange multipliers $L_{Z}$ to handle the constraint $\sum_{k \neq Z} e^{g(k)} G_{Z, k}=\sum_{k \neq Z} G_{Z, k}$ allows the optimization to be written in an unconstrained form:

$$
\begin{aligned}
\inf _{g}\left\{\sum_{k=1}^{n} e^{g(k)} G_{Z, k}\left(h_{q, k}^{\varphi_{P}}-h_{q, Z}^{\varphi_{P}}\right)\right. & +\frac{1}{\varphi_{P}} \sum_{k \neq Z}\left(e^{g(k)}(g(k)-1)+1\right) G_{Z, k} \\
& \left.+L_{Z}\left(\sum_{k \neq Z} e^{g(k)} G_{Z, k}-\sum_{k \neq Z} G_{Z, k}\right)\right\}
\end{aligned}
$$

First order conditions in $g(k)$ give:

$$
\begin{aligned}
0 & =e^{g(k)} G_{Z, k}\left(h_{q, k}^{\varphi_{P}}-h_{q, Z}^{\varphi_{P}}\right)+\frac{1}{\varphi_{P}} e^{g(k)} g(k) G_{Z, k}+L_{Z} e^{g(k)} G_{Z, k} \\
0 & =h_{q, k}^{\varphi_{P}}-h_{q, Z}^{\varphi_{P}}+\frac{1}{\varphi_{P}} g(k)+L_{Z} \\
g(k) & =-\varphi_{P} L_{Z}-\varphi_{P}\left(h_{q, k}^{\varphi_{P}}-h_{q, Z}^{\varphi_{P}}\right)
\end{aligned}
$$

This expression is substituted into the constraint in order to solve for $L_{Z}$ :

$$
\begin{aligned}
\sum_{k \neq Z} e^{g(k)} G_{Z, k} & =\sum_{k \neq Z} G_{Z, k} \\
\sum_{k \neq Z} e^{-\varphi_{P} L_{Z}-\varphi_{P}\left(h_{q, k}^{\varphi_{P}}-h_{q, Z}^{\varphi_{P}}\right)} G_{Z, k} & =\sum_{k \neq Z} G_{Z, k} \\
e^{-\varphi_{P} L_{Z}} & =\frac{\sum_{k \neq Z} G_{Z, k}}{\sum_{k \neq Z} e^{-\varphi_{P}\left(h_{q, k}^{\varphi_{P}}-h_{q, Z}^{\varphi_{P}}\right)} G_{Z, k}} \\
L_{Z} & =\frac{1}{\varphi_{P}} \log \left(\frac{\sum_{k \neq Z} e^{-\varphi_{P}\left(h_{q, k}^{\varphi_{P}}-h_{q, Z}^{\left.\varphi_{P}\right)}\right.} G_{Z, k}}{\sum_{k \neq Z} G_{Z, k}}\right)
\end{aligned}
$$

Substituting this back into equation (B.34) yields:

$$
g(k)=-\log \left(\frac{\sum_{k \neq Z} e^{-\varphi_{P}\left(h_{q, k}^{\varphi_{P}}-h_{q, Z}^{\left.\varphi_{P}\right)}\right.} G_{Z, k}}{\sum_{k \neq Z} G_{Z, k}}\right)-\varphi_{P}\left(h_{q, k}^{\varphi_{P}}-h_{q, Z}^{\varphi_{P}}\right)
$$

Verify that $g$ provides a minimum: To show that this expression for $g$ yields a 
minimum, let $f$ be any other function that satisfies:

$$
\sum_{k \neq Z} e^{f(k)} G_{Z, k}=\sum_{k \neq Z} G_{Z, k}
$$

Define $m(k)=e^{f(k)}-e^{g(k)}$, and then define $f_{\epsilon}(k)=\log \left(\epsilon m(k)+e^{g(k)}\right)$ for $\epsilon \in[0,1]$. Then $f_{\epsilon} \in \mathcal{G}_{Z}$ for all $\epsilon$, and:

$$
\sum_{k \neq Z} m(k) G_{Z, k}=0
$$

Substituting $f_{\epsilon}$ into the term to be optimized gives an expression that depends on $\epsilon$ :

$$
\begin{aligned}
& \sum_{k=1}^{n}\left(\epsilon m(k)+e^{g(k)}\right) G_{Z, k}\left(h_{q, k}^{\varphi_{P}}-h_{q, Z}^{\varphi_{P}}\right) \\
& \quad+\frac{1}{\varphi_{P}} \sum_{k \neq Z}\left(\left(\epsilon m(k)+e^{g(k)}\right)\left(\log \left(\epsilon m(k)+e^{g(k)}\right)-1\right)+1\right) G_{Z, k}
\end{aligned}
$$

When derivatives with respect to $\epsilon$ are considered, this function will be shown to have zero first derivative at $\epsilon=0$, and positive second derivative for $\epsilon \in[0,1]$, thus showing that the expression for $g$ does indeed yield a minimum. Taking a first derivative in $\epsilon$ gives:

$$
\begin{aligned}
& \sum_{k=1}^{n} m(k) G_{Z, k}\left(h_{q, k}^{\varphi_{P}}-h_{q, Z}^{\varphi_{P}}\right)+\frac{1}{\varphi_{P}} \sum_{k \neq Z} m(k)\left(\log \left(\epsilon m(k)+e^{g(k)}\right)-1\right) G_{Z, k}+\frac{1}{\varphi_{P}} \sum_{k \neq Z} m(k) G_{Z, k} \\
= & \sum_{k=1}^{n} m(k) G_{Z, k}\left(h_{q, k}^{\varphi_{P}}-h_{q, Z}^{\varphi_{P}}\right)+\frac{1}{\varphi_{P}} \sum_{k \neq Z} m(k) \log \left(\epsilon m(k)+e^{g(k)}\right) G_{Z, k}
\end{aligned}
$$

Evaluating at $\epsilon=0$ and substituting the expression for $g$ shows that this term vanishes as expected. The second derivative gives:

$$
\frac{1}{\varphi_{P}} \sum_{k \neq Z} \frac{m^{2}(k)}{\epsilon m(k)+e^{g(k)}} G_{Z, k}=\frac{1}{\varphi_{P}} \sum_{k \neq Z} \frac{m^{2}(k)}{e^{f_{\epsilon}(k)}} G_{Z, k}
$$

which is clearly positive for all $\epsilon \in[0,1]$. This shows that $g$ provides a minimum. 


\section{Bibliography}

Almgren, R. (2003, January). Optimal execution with nonlinear impact functions and trading-enhanced risk. Applied Mathematical Finance 10(1), 1-18.

Almgren, R. and N. Chriss (2000). Optimal Execution of Portfolio Transactions. Journal of Risk $3(2), 5-39$.

Avellaneda, M. and S. Stoikov (2008, November). High-frequency trading in a limit order book. Quantitative Finance 8(3), 217-224.

Bayraktar, E., Y.-J. Huang, and Z. Zhou (2014). ON HEDGING AMERICAN OPTIONS UNDER MODEL UNCERTAINTY. http://arxiv.org/abs/1309.2982.

Bayraktar, E. and M. Ludkovski (2011). Optimal trade execution in illiquid markets. Mathematical Finance 21(4), 681-701.

Bayraktar, E. and M. Ludkovski (2012). Liquidation in limit order books with controlled intensity. Mathematical Finance, http://dx.doi.org/10.1111/j.1467-9965.2012.00529.x.

Carmona, R. and K. Webster (2012a). A belief-driven order book model. http://citeseerx.ist.psu.edu/viewdoc/summary?doi=10.1.1.380.1304.

Carmona, R. and K. Webster (2012b). High frequency market making. http://arxiv.org/abs/1210.5781.

Cartea, Á. and S. Jaimungal (2012). Risk metrics and fine tuning of high frequency trading strategies. Forthcoming in Mathematical Finance.

Cartea, Á. and S. Jaimungal (2013). Modeling asset prices for algorithmic and high frequency trading. Forthcoming in Applied Mathematical Finance.

Cartea, Á. and S. Jaimungal (2014). Optimal execution with limit and market orders. $\underline{\text { SSRN eLibrary, http://ssrn.com/abstract }=2397805 .}$ 
Cartea, Á., S. Jaimungal, and D. Kinzebulatov (2013). Algorithmic trading with learning. $\underline{\text { SSRN eLibrary, http://ssrn.com/paper }=2373196 .}$

Cartea, Á., S. Jaimungal, and J. Ricci (2014). Buy low, sell high: A high frequency trading perspective. SIAM Journal on Mathematical Finance $\underline{5}$ (1), 415-444.

Cartea, Á. and J. Penalva (2011). Where is the value in high frequency trading? SSRN eLibrary, forthcoming Quarterly Journal of Finance, http://ssrn.com/paper=1712765.

Cebirog̃lu, G. and U. Horst (2013). Optimal order exposure and the market impact of limit orders. SSRN eLibrary, http://ssrn.com/paper=1997092.

Cont, R. (2006). MODEL UNCERTAINTY AND ITS IMPACT ON THE PRICING OF DERIVATIVE INSTRUMENTS. Mathematical Finanace 16(3), 519-547.

Cont, R., S. Stoikov, and R. Talreja (2010). A Stochastic Model for Order Book Dynamics. Operations Research 58(3), 549-563.

Ellsberg, D. (1961). Risk, Ambiguity, and the Savage Axioms. The Quarterly Journal of Economics 75, 643 .

Fahim, A. E. and Y.-J. Huang (2014). Model-independent superhedging under portfolio constraints. http://arxiv.org/abs/1402.2599.

Fleming, W. H. and H. M. Soner (2006). Controlled Markov Processes and Viscosity Solutions (2nd ed.). Springer.

Fodra, P. and M. Labadie (2012). High-frequency market-making with inventory constraints and directional bets. Working papers, HAL.

Guéant, O., C.-A. Lehalle, and J. Fernandez-Tapia (2012). Optimal portfolio liquidation with limit orders. SIAM Journal on Financial Mathematics 3(1), 740-764.

Guéant, O., C.-A. Lehalle, and J. Fernandez-Tapia (2013). Dealing with the inventory risk: a solution to the market making problem. Mathematics and Financial Economics 7(4), 477-507.

Guéant, O. and G. Royer (2014). VWAP execution and guaranteed VWAP. SIAM Journal on Mathematical Finance 5(1), 445-471.

Guilbaud, F. and H. Pham (2013a). Optimal high-frequency trading in a pro rata microstructure with predictive information. Mathematical Finance, n/a-n/a. 
Guilbaud, F. and H. Pham (2013b). Optimal high frequency trading with limit and market orders. Quantitative Finance 13(1), 79-94.

Horst, U. and F. Naujokat (2014). When to cross the spread: Trading in two-side limit order books. SIAM Journal on Mathematical Finance 5(1), 278-315.

Horst, U. and C. Rothe (2008). Queuing, social interactions and the microstructure of financial markets. Macroeconomic Dynamics $\underline{12}$, 211-233.

Jacod, J. and A. N. Shiryaev (1987). Limit theorems for stochastic processes. Grundlehren der mathematischen Wissenschaften. Springer-Verlag.

Jaimungal, S. (2011). Irreversible Investments and Ambiguity Aversion. SSRN eLibrary, http://ssrn.com/abstract $=1961786$.

Jaimungal, S. and D. Kinzebulatov (2013). Optimal Execution with a Price Limiter. SSRN eLibrary, http://ssrn.com/abstract=2199889.

Jaimungal, S., D. Kinzebulatov, and D. Rubisov (2013). Optimal accelerated share repurchase. SSRN eLibrary, http://ssrn.com/paper $=2360394$.

Jaimungal, S. and G. Sigloch (2012). Incorporating risk and ambiguity aversion into a hybrid model of default. Mathematical Finance $\underline{22}(1), 57-81$.

Keynes, J. M. (1921). A Treatise on Probability. MacMillan and Co. Limited.

Kharroubi, I. and H. Pham (2010). Optimal portfolio liquidation with execution cost and risk. SIAM Journal on Financial Mathematics 1, 897-931.

Knight, F. H. (1921). Risk, Uncertainty, and Profit. Houghton Mifflin Company.

Lim, A. E. B. and J. G. Shanthikumar (2007). Relative Entropy, Exponential Utility, and Robust Dynamic Pricing. Operations Research 55(2), 198-214.

Øksendal, B. and S. Mataramvura (2008). Risk minimizing portfolios and HJBI equations for stochastic differential games. Stochastics 80(4), 317-337.

Øksendal, B. and A. Sulem (2009). Applied Stochastic Control of Jump Diffusions (Third ed.). Stochastic Modelling and Applied Probability. Berlin Heidelberg: Springer-Verlag.

Parlour, C. A. (1998). Price dynamics in limit order markets. The Review of Financial $\underline{\text { Studies }} \underline{11}(4), 789-816$. 
Pham, H. (2009). Continuous-time Stochastic Control and Optimization with Financial Applications (1st ed.), Volume 61 of Stochastic Modelling and Applied Probability. Berlin Heidelberg: Springer-Verlag.

Yong, J. and X. Y. Zhou (1999). Stochastic Controls Hamiltonian Systems and HJB Equations, Volume 43 of Stochastic Modelling and Applied Probability. Berlin Heidelberg: Springer-Verlag. 